San Jose State University

SJSU ScholarWorks

Master's Theses

Master's Theses and Graduate Research

Spring 2013

\title{
Characterization of Hydride-Based Silica and Diamond Hydride Stationary Phases
}

Nima C. Parmar

San Jose State University

Follow this and additional works at: https://scholarworks.sjsu.edu/etd_theses

\section{Recommended Citation}

Parmar, Nima C., "Characterization of Hydride-Based Silica and Diamond Hydride Stationary Phases" (2013). Master's Theses. 4306.

DOI: https://doi.org/10.31979/etd.b33r-xhty

https://scholarworks.sjsu.edu/etd_theses/4306

This Thesis is brought to you for free and open access by the Master's Theses and Graduate Research at SJSU ScholarWorks. It has been accepted for inclusion in Master's Theses by an authorized administrator of SJSU ScholarWorks. For more information, please contact scholarworks@sjsu.edu. 


\title{
CHARACTERIZATION OF HYDRIDE-BASED SILICA AND DIAMOND HYDRIDE STATIONARY PHASES
}

\author{
A Thesis \\ Presented to \\ The Faculty of the Department of Chemistry \\ San José State University \\ In Partial Fulfillment \\ of the Requirements for the Degree \\ Master of Science
}

by

Nima Parmar

May 2013 
(C) 2013

Nima Parmar

ALL RIGHTS RESERVED 
The Designated Thesis Committee Approves the Thesis Titled

\title{
CHARACTERIZATION OF HYDRIDE-BASED SILICA AND DIAMOND HYDRIDE STATIONARY PHASES
}

\author{
by \\ Nima Parmar \\ APPROVED FOR THE DEPARTMENT OF CHEMISTRY \\ SAN JOSÉ STATE UNIVERSITY
}

May 2013
Dr. Joseph J. Pesek
Chemistry Department
Dr. Roger H. Terrill
Chemistry Department
Dr. Brooke Lustig
Chemistry Department 


\section{ABSTRACT \\ CHARACTERIZATION OF HYDRIDE-BASED SILICA AND DIAMOND HYDRIDE STATIONARY PHASES \\ by Nima Parmar}

Aqueous normal phase chromatography possesses characteristics of both normal phase (NP) and also reverse phase (RP) chromatography. It can retain polar as well as non-polar compounds by carefully selecting the concentration ratio of organic solvent to water in the mobile phase. Silica hydride columns contain only a small percentage of silanol groups which are polar in nature and have an effect on the separation process. Diamond hydride columns consist of a small amount ( $2 \%)$ of carbon chemically bonded to a silica hydride surface.

This research work characterized both hydride-based silica and diamond hydride stationary phases under isocratic and gradient conditions. Both columns were compared using the same conditions, and their efficiencies and symmetries were calculated. Diamond hydride showed different selectivity than the silica hydride column because of the $2 \%$ carbon on the silica hydride surface. This research work shows that both the silica hydride and diamond hydride columns can be used in aqueous normal phase conditions. 


\section{ACKNOWLEDGEMENTS}

I would like to thank Dr. Joseph J. Pesek for giving me an opportunity to work on this project and for his valuable guidance throughout my research work. I am grateful to Dr. Maria T. Matyska for her support and guidance during my entire work on this project. I would like to thank Dr. Roger H. Terrill and Dr. Brooke Lustig for being my committee members and giving their valuable comments on my thesis. I would also like to thank the chemistry department staff; especially Mr. Michael Stephens, the lead technician and Mr. Craig Wood, the equipment technician for their help with instrument related problems whenever needed. I am deeply thankful to my parents, my brother, my friend Prema and my in-laws for their continued support. I am immensely thankful to my little one Parin Bhajiwala (19 months) for sharing his playtime with me to finish up my thesis. Last but not the least; I would like to acknowledge my husband Jigar Bhajiwala for his understanding and encouragement throughout my research work. I cannot think of finishing this thesis without his immense support and patience. 


\section{TABLE OF CONTENTS}

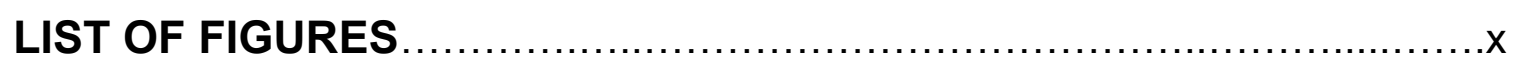

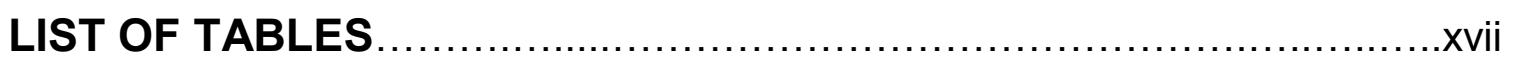

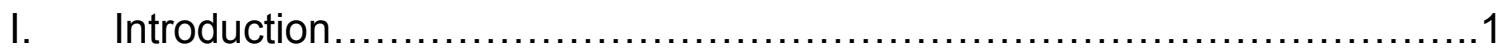

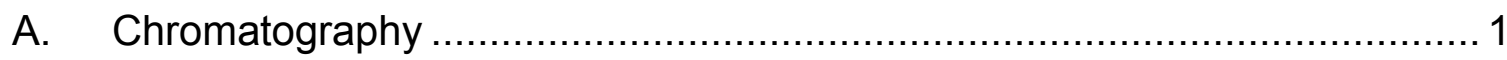

B. Theory of High Performance Liquid Chromatography (HPLC) .................. 2

C. Aqueous Normal Phase Chromatography (ANP) …............................. 5

D. Resolution, Selectivity and Number of Theoretical Plates ......................... 6

E. Silica Surface: Surface Modification...................................................... 10

F. Advantages of Using Hydride-based Stationary Phases.......................... 16

G. High Performance Liquid Chromatography (HPLC) …........................... 18

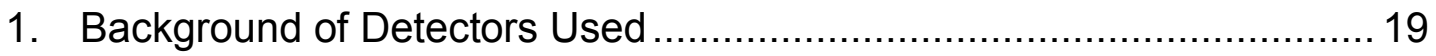

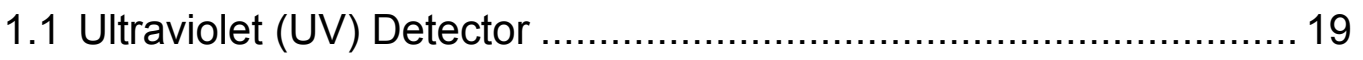

1.2 Evaporative Light Scattering Detector (ELSD) …........................ 20

H. Types of Samples Used in the Evaluation of HPLC Columns ...................2 24

I. Silica Hydride and Diamond Hydride Stationary Phases ......................... 26

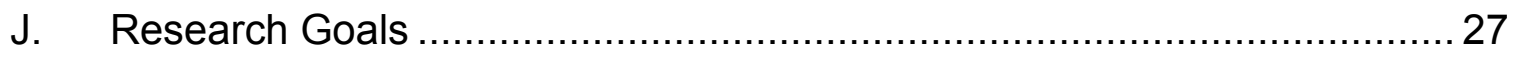

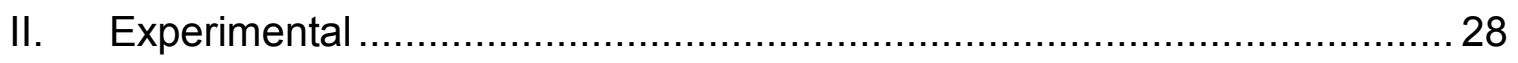

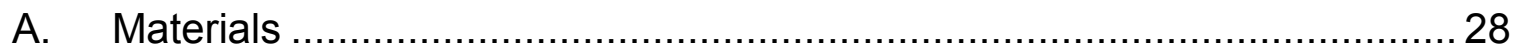

1.1. Compounds Used for the Characterization of Columns ................. 28

1.2. Structures of Compounds Used for Chromatographic

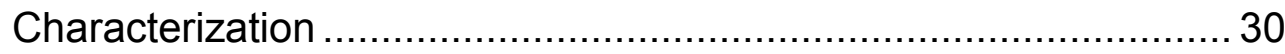


B. Instrumentation and Operation Procedure …....................................... 32

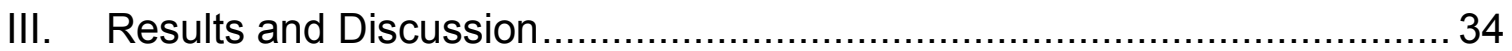

A. Chromatographic Evaluation of HPLC Columns ................................. 34

1. ANP Behavior of Si-H Column Using an ELSD Detector ...................... 35

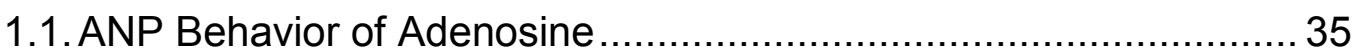

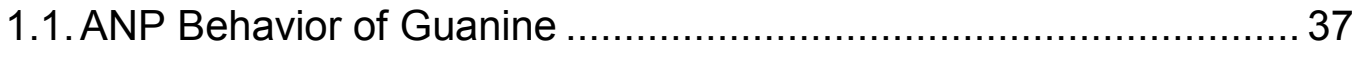

1.2. ANP Behavior of L-Tryptophan ................................................ 40

1.4. ANP Behavior of Guanidine …............................................... 42

1.5. ANP Behavior of Creatinine ...................................................... 43

2. ANP Behavior of Si-H Column Using UV Detector ............................. 45

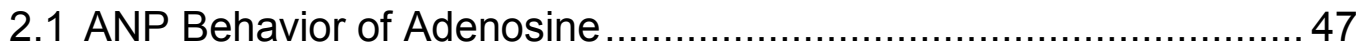

2.2. ANP Behavior of Guanine ......................................................... 50

2.3. ANP Behavior of Creatinine …................................................... 53

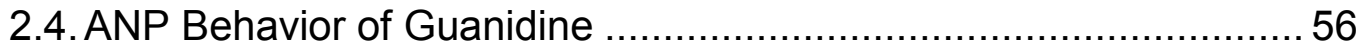

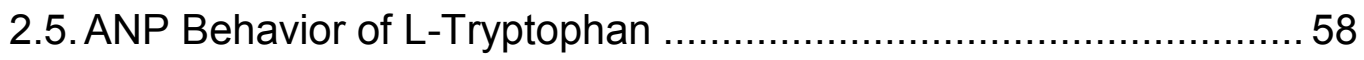

2.6. ANP Isocratic Behavior of Primary Amines .................................. 59

3.0 ANP Behavior of Si-H Column in Gradient Mode Using UV Detector ... 64

3.1 ANP Separation of Two Compounds Using a Gradient Method ...... 68

3.2. ANP Separation of Three Compounds Using Gradient Method ...... 71

3.3. ANP Separation of Four Compounds Using Gradient Method ........ 74

4.0 ANP Behavior of the Diamond hydride Column Using the ELSD

Detector 


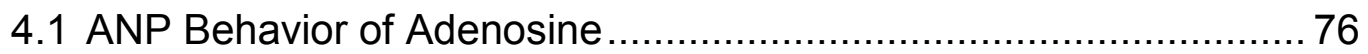

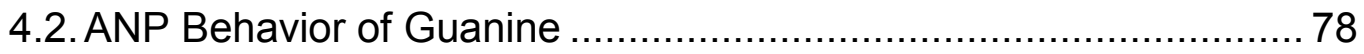

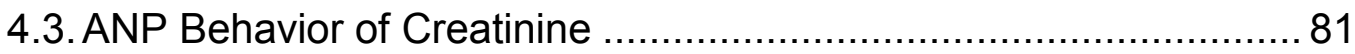

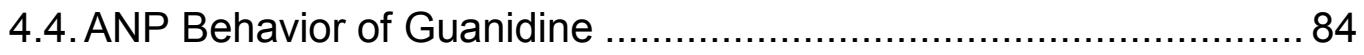

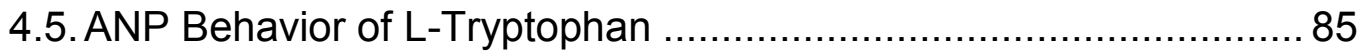

5.0 ANP Behavior of Diamond hydride Column Using a UV Detector ......... 87

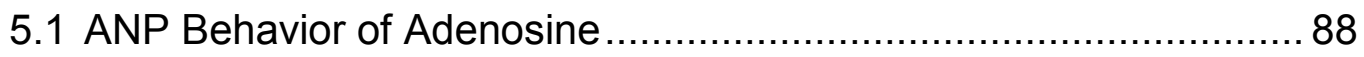

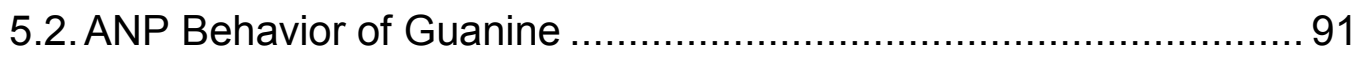

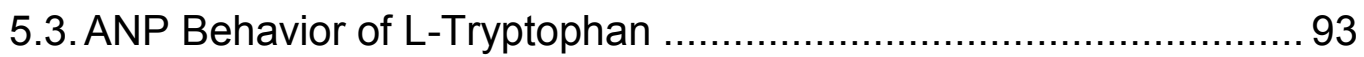

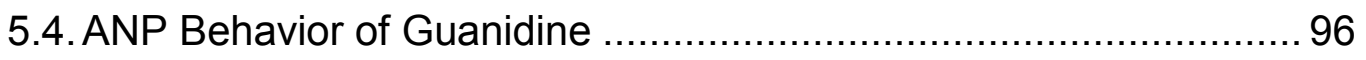

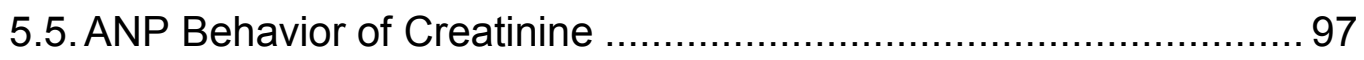

5.6. ANP Isocratic Behavior of Primary Amines .................................... 99

6.0 ANP Behavior of Diamond Hydride Column with Gradient Elution Using UV Detection ................................................. 103

6.1 ANP Separation of Two Compounds Using Gradient Method....... 106 6.2. ANP Separation of Three Compounds Using Gradient Method .... 109

6.3. ANP Separation of Four Compounds Using Gradient Method ...... 112

7.0 Comparison of the Silica Hydride and Diamond Hydride Columns Under ANP Conditions ............................................... 114

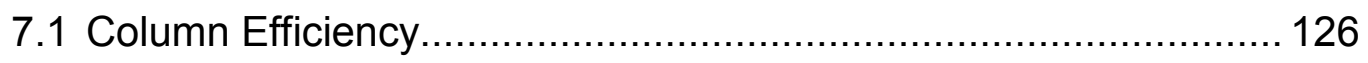

7.2. Peak Symmetry Factor ........................................................... 126

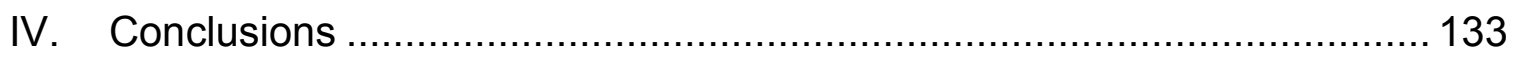




\section{REFERENCES}




\section{LIST OF FIGURES}

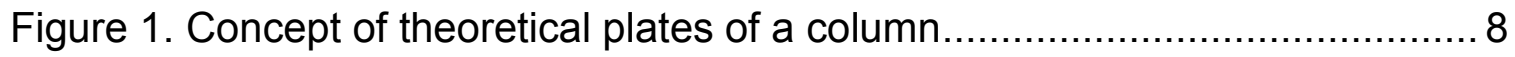

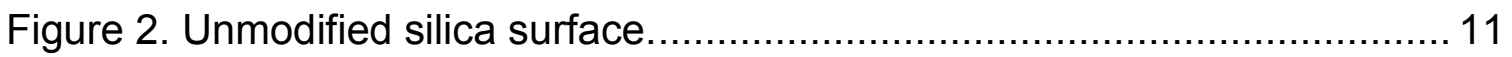

Figure 3. Active groups found on the silica surface. .............................. 12

Figure 4. Various methods of silica surface modification. .......................... 15

Figure 5. Ideal structures of a native silica surface (left) and silica hydride surface (right) [17]. ......................................................... 17

Figure 6. Schematic diagram of a HPLC system. ............................... 19

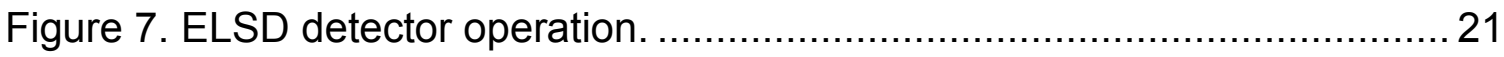

Figure 8. A schematic diagram of thermo-split technology. A. Cooled surface.

B. Heated surface................................................................... 23

Figure 9. Structures of compounds used for chromatographic characterization. 31

Figure 10. Chromatogram of adenosine on the silica hydride stationary phase at $80: 20$ acetonitrile/water mobile phase (both premixed with $0.1 \%$

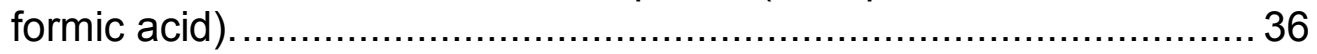

Figure 11. Retention map of adenosine on the silica hydride column using ELSD.

Figure 12. Chromatograms of guanine obtained using the silica hydride stationary phase 70:30 acetonitrile/water (Top) and 80:20 acetonitrile/water (Bottom) mobile phase (both premixed with $0.1 \%$ formic acid).

Figure 13. Retention map of guanine on the silica hydride using ELSD.

Figure 14. Chromatogram of L-tryptophan on the silica hydride stationary phase at 50:50 acetonitrile/water mobile phase (both premixed with $0.1 \%$ formic acid).

Figure 15. Retention map of L-tryptophan on the silica hydride using ELSD..... 41

Figure 16. Retention map of guanidine on the silica hydride column............... 43 
Figure 17. Retention map of creatinine on the silica hydride column using ELSD.

Figure 18. Chromatograms of adenosine obtained using the silica hydride column at 50:50 acetonitrile/water (Top), 80:20 acetonitrile/water (Bottom) as mobile phase (both premixed with $0.1 \%$ formic acid) ..... 48

Figure 19. Retention map of adenosine on the silica hydride column using UV detection.

Figure 20. Chromatograms of guanine obtained using the silica hydride stationary phase at 50:50 acetonitrile/water (Top) and 80:20 acetonitrile/water (Bottom) as mobile phase (both premixed with $0.1 \%$ formic acid).

Figure 21. Retention map of guanine on the silica hydride column using UV detection.

Figure 22. Chromatogram of creatinine on the silica hydride stationary phase. Mobile phase: $50: 50$ acetonitrile $+0.1 \%$ formic acid / water $+0.1 \%$ formic acid.

Figure 23. Retention map of creatinine on the silica hydride column using UV detection.

Figure 24. Chromatogram of guanidine on the silica hydride stationary phase. Mobile phase: $50: 50$ acetonitrile $+0.1 \%$ formic acid / water $+0.1 \%$ formic acid.

Figure 25. Retention map of guanidine on the silica hydride column using UV detection.

Figure 26. Chromatogram of L-tryptophan on the silica hydride stationary phase. Mobile phase: $80: 20$ acetonitrile $+0.1 \%$ formic acid / water $+0.1 \%$ formic acid.

Figure 27. Retention map of L-tryptophan on the silica hydride column using UV detection.

Figure 28. Chromatogram of 2,6-diaminopurine on the silica hydride stationary phase. Mobile phase: $80: 20$ acetonitrile $+0.1 \%$ formic acid / water $+0.1 \%$ formic acid. 
Figure 29. Chromatogram of benzylamine on the silica hydride stationary phase. Mobile phase: $80: 20$ acetonitrile $+0.1 \%$ formic acid / water $+0.1 \%$ formic acid.

Figure 30. Chromatogram of 2-phenethylamine on the silica hydride stationary phase. Mobile phase: $80: 20$ acetonitrile $+0.1 \%$ formic acid / water $+0.1 \%$ formic acid.

Figure 31. Chromatogram of 1,2,4,5-benzenetetracarboxamide on the silica hydride stationary phase. Mobile phase: $80: 20$ acetonitrile $+0.1 \%$ formic acid / water $+0.1 \%$ formic acid.

Figure 32. Chromatogram of 2,6-diaminopurine on the silica hydride column. Mobile phase Gradient B.

Figure 33. Chromatogram of 1,2,4,5-benzenetetramine tetrahydrochloride on the silica hydride column. Mobile phase Gradient A. 66

Figure 34. Chromatogram of 4-Chloro-1,2-phenylenediamine on the silica hydride column. Mobile phase Gradient

Figure 35 . Aqueous normal phase gradient separation of 1,2,4,5benzenetetramine tetrahydrochloride and 2,6-diaminopurine on the silica hydride column. The peak at $3.39 \mathrm{~min}$ is for $1,2,4,5-$ benzenetetramine tetrahydrochloride and the peak at $10.65 \mathrm{~min}$ is for 2,6-diaminopurine. Mobile phase Gradient A.

Figure 36. Aqueous normal phase gradient separation of 2-phenethylamine and 2,6-diaminopurine on the silica hydride column. The peak at $4.46 \mathrm{~min}$ is for 2-phenethylamine and the peak at $6.08 \mathrm{~min}$ is for 2,6-diaminopurine.Mobile phase Gradient B.

Figure 37. Aqueous normal phase gradient separation of benzylamine and 2,6-diaminopurine on the silica hydride column. The peak at 11.38 $\mathrm{min}$ is for benzylamine and the peak at $12.87 \mathrm{~min}$ is for 2,6diaminopurine. Mobile phase Gradient $D$....

Figure 38. Aqueous normal phase gradient separation of 1,2,4,5benzenetetramine tetrahydrochloride, benzylamine and 2,6diaminopurine on the silica hydride column. The peak at $3.42 \mathrm{~min}$ is for 1,2,4,5-benzenetetramine tetrahydrochloride, peak at 9.52 min is for benzylamine and the peak at $10.94 \mathrm{~min}$ is for 2,6diaminopurine. Mobile phase Gradient A 
Figure 39. Aqueous normal phase gradient separation of 1,2,4,5benzenetetramine tetrahydrochloride, 2-phenethylamine and 2,6diaminopurine on the silica hydride column. The peak at $3.41 \mathrm{~min}$ is for 1,2,4,5-benzenetetramine tetrahydrochloride, peak at 9.33 $\min$ is for 2-phenethylamine and the peak at $10.84 \mathrm{~min}$ is for 2,6-diaminopurine. Mobile phase Gradient A.

Figure 40. Aqueous normal phase gradient separation of 1,2,4,5benzenetetramine tetrahydrochloride, benzylamine, 2phenethylamine and 2,6-diaminopurine on the silica hydride column. Peak identification: $1=1,2,4,5$-benzenetetramine tetrahydrochloride, peak $2+3=$ benzylamine, 2-phenethylamine, peak $4=2,6$-diaminopurine. Mobile phase Gradient A

Figure 41. Chromatograms of adenosine obtained using the diamond hydride stationary phase at 50:50 acetonitrile/water (Top) and 80:20 acetonitrile/water (Bottom) mobile phase (both premixed with $0.1 \%$ formic acid).

Figure 42. Retention map of adenosine on the diamond hydride column using ELSD

Figure 43. Chromatograms of guanine obtained using the diamond hydride stationary phase at 70:30 acetonitrile/water (Top) and 80:20 acetonitrile/water (Bottom) mobile phase (both premixed with $0.1 \%$ formic acid).

Figure 44. Retention map of guanine on the diamond hydride column using ELSD.

Figure 45. Chromatograms of creatinine obtained using the diamond hydride stationary phase at 50:50 acetonitrile/water (Top) and 80:20 acetonitrile/water (Bottom) mobile phase (both premixed with $0.1 \%$ formic acid).

Figure 46. Retention map of creatinine on the diamond hydride column using ELSD.

Figure 47. Retention map of guanidine on the diamond hydride column using ELSD.

Figure 48. Retention map of L-tryptophan on the diamond hydride column using ELSD. 
Figure 49. Chromatograms of adenosine obtained using the diamond hydride stationary phase at 60:40 acetonitrile/water (Top) and 90:10 acetonitrile/water (Bottom) mobile phase (both premixed with $0.1 \%$ formic acid).

Figure 50. Retention map of adenosine on the diamond hydride column using UV detection.

Figure 51. Chromatograms of guanine obtained on the diamond hydride stationary phase. A. 60:40 acetonitrile/water with $0.1 \%$ FA. B. 80:20 acetonitrile/water with 0.1\% FA. C. 90:10 acetonitrile/water mobile phase with $0.1 \%$ FA.

Figure 52. Retention map of guanine on the diamond hydride column using UV detection.

Figure 53. Chromatogram of L-tryptophan on the diamond hydride stationary phase. Mobile phase: $90: 10$ acetonitrile $+0.1 \%$ formic acid / water $+0.1 \%$ formic acid.

Figure 54. Retention map of L-tryptophan on the diamond hydride column using UV detection.

Figure 55. Retention map of guanidine on the diamond hydride column using UV detection.

Figure 56. Retention map of creatinine on the diamond hydride column using UV detection.

Figure 57. Chromatogram of 2-phenethylamine on the diamond hydride stationary phase. Mobile phase: $80: 20$ acetonitrile $+0.1 \%$ formic acid / water $+0.1 \%$ formic acid.

Figure 58. Chromatogram of benzylamine on the diamond hydride stationary phase. Mobile phase: $80: 20$ acetonitrile $+0.1 \%$ formic acid / water $+0.1 \%$ formic acid.

Figure 59. Chromatogram of 2,6-diaminopurine on the diamond hydride stationary phase. Mobile phase: $80: 20$ acetonitrile $+0.1 \%$ formic acid / water $+0.1 \%$ formic acid. 100

Figure 60. Chromatogram of 2,6-diaminopurine on the diamond hydride column. Mobile phase Gradient B. 
Figure 61. Chromatogram of 1,2,4,5-benzenetetramine tetrahydrochloride on the diamond hydride column. Mobile phase Gradient A. 105

Figure 62. Aqueous normal phase gradient separation of 1,2,4,5benzenetetramine tetrahydrochloride and 2,6-diaminopurine on the diamond hydride column. The peak at 3.42 minutes is for 1,2,4,5-benzenetetramine tetrahydrochloride and the peak at 11.05 minutes is for 2,6-diaminopurine. Mobile phase Gradient A. . 107

Figure 63. Aqueous normal phase gradient separation of 1,2,4,5benzenetetramine tetrahydrochloride and 2,6-diaminopurine on the diamond hydride column. The peak at 2.60 minutes is for 1,2,4,5-benzenetetramine tetrahydrochloride and the peak at 6.60 minutes is for 2,6-diaminopurine.Mobile phase Gradient B. .... 108

Figure 64. Aqueous normal phase gradient separation of 2-phenethylamine and 2,6-diaminopurine on the diamond hydride column. The peak at 9.94 minutes is for 2-phenethylamine and the peak at 11.25 minutes is for 2,6- diaminopurine. Mobile phase Gradient A. 109

Figure 65. Aqueous normal phase gradient separation of 1,2,4,5benzenetetramine tetrahydrochloride, benzylamine and 2,6diaminopurine on the diamond hydride column. The peak at 3.43 minutes is for 1,2,4,5-benzenetetramine tetrahydrochloride, peak at 9.53 minutes is for benzylamine and the peak at 10.78 minutes is for 2,6-diaminopurine. Mobile phase Gradient A.

Figure 66. Aqueous normal phase gradient separation of 1,2,4,5benzenetetramine tetrahydrochloride, 2-phenethylamine and 2,6-diaminopurine on the diamond hydride column. The peak at 4.51 minutes is for 1,2,4,5-benzenetetramine tetrahydrochloride, peak at 11.27 minutes is for 2-phenethylamine and the peak at 12.4 minutes is for 2,6-daminopurine. Mobile phase Gradient D..... 111

Figure 67. ANP gradient separation of 4-chloro-1,2-phenylenediamine, 1,2,4,5-benzenetetramine tetrahydrochloride, benzylamine and 2,6-diaminopurine on the diamond hydride column. Peak identification: $1+2=4$-chloro-1,2-phenylenediamine and 1,2,4,5benzenetetramine tetrahydrochloride, peak $3=$ benzylamine, peak $4=2,6$-daminopurine. Mobile phase Gradient A.

Figure 68. Comparison of retention maps of adenosine on the silica hydride and diamond hydride columns using UV detection. 
Figure 69. Comparison of retention maps of creatinine on the silica hydride and diamond hydride columns using UV detection.

Figure 70. Comparison of retention maps of guanidine on the silica hydride and diamond hydride columns using UV detection.

Figure 71. Comparison of retention maps of L-tryptophan on the silica hydride and diamond hydride columns using UV detection.

Figure 72. Comparison of retention maps of guanine on the silica hydride and diamond hydride columns using UV detection.

Figure 73. Chromatogram of benzylamine on the silica hydride and diamond hydride columns. Mobile phase Gradient D.

Figure 74. Chromatograms of 4-chloro-1,2-phenylenediamine on the silica hydride and diamond hydride columns. Mobile phase Gradient D. . 120

Figure 75. Separation of benzylamine and 2,6-diaminopurine on the silica hydride and diamond hydride columns. Mobile phase Gradient D. . 121

Figure 76. Separation of 1,2,4,5-benzenetetramine tetrahydrochloride, 2phenethylamine and 2,6-diaminopurine on the silica hydride and diamond hydride columns. Mobile phase Gradient B.

Figure 77. Separation of 1,2,4,5-benzenetetramine tetrahydrochloride and 2-phenethylamine on the silica hydride and diamond hydride columns. Mobile phase Gradient B.

Figure 78. Separation of 1,2,4,5-benzenetetramine tetrahydrochloride and 2,6--diaminopurine on the silica hydride and diamond hydride columns. Mobile phase Gradient A

Figure 79. Determination of peak asymmetry factor.

Figure 80. Separation of 2-phenethylamine and 2,6-diaminopurine on the silica hydride and diamond hydride columns. Mobile phase Gradient B.

Figure 81. Separation of 1,2,4,5-benzenetetramine tetrahydrochloride and 2,6-diaminopurine on the silica hydride and diamond hydride columns. Mobile phase Gradient B.... 


\section{LIST OF TABLES}

Table 1. Compounds used in the mobile phase and for the preparation of the samples.

Table 2. Compounds used for the evaluation of columns............................... 29

Table 3. Retention time of adenosine on the silica hydride column using ELSD.37

Table 4. Retention time of guanine on the silica hydride column using ELSD....39

Table 5. Retention time of L-tryptophan on the silica hydride using ELSD. ........ 41

Table 6. Retention time of guanidine on the silica hydride using ELSD............. 42

Table 7. Retention time of creatinine on the silica hydride column using ELSD. 44

Table 8. Mobile phase Gradients A and B used for ANP analysis................... 46

Table 9. Mobile phase Gradients C and D used for ANP analysis................... 47

Table 10. Retention time of adenosine on the silica hydride column using UV

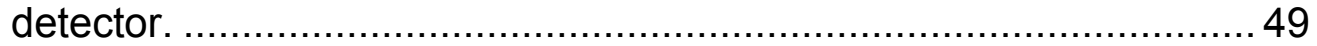

Table 11. Retention time of guanine on the silica hydride column using UV

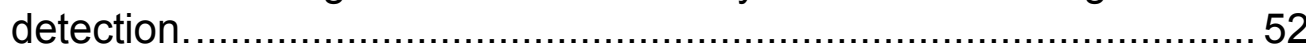

Table 12. Retention time of creatinine on the silica hydride column using UV detector.

Table 13. Retention time of guanidine on the silica hydride column using UV detector.

Table 14. Retention time of L-tryptophan on the silica hydride column using UV detector.

Table 15. Summary of isocratic elution data for primary amines. ..................... 62

Table 16. Summary of isocratic elution data for Primary amines...................... 63

Table 17. Summary of isocratic elution data for 3,4-diaminobenzophenone. ..... 64

Table 18. Retention times of primary amines on the silica hydride column. ....... 68 
Table 19. Retention time of adenosine on the diamond hydride column using ELSD.

Table 20. Retention time of guanine on the diamond hydride using ELSD 80

Table 21. Retention time of creatinine on the diamond hydride column using ELSD.

Table 22. Retention time of guanidine on the diamond hydride column using ELSD.

Table 23. Retention time of L-tryptophan on the diamond hydride column using ELSD.

Table 24. Retention time of adenosine on the diamond hydride column using UV detector.

Table 25. Retention time of guanine on the diamond hydride column using UV detector.

Table 26. Retention time of L-tryptophan on the diamond hydride column using UV detection.

Table 27. Retention time of guanidine on the diamond hydride column using UV detection.

Table 28. Retention time of creatinine on the diamond hydride column using UV detection.

Table 29. Summary of the retention times of primary amines on the diamond hydride column using UV.

Table 30. Summary of the retention times of primary amines on the diamond hydride column using UV.

Table 31. Summary of isocratic elution data for 3,4-diaminobenzophenone. ... 102

Table 32. ANP gradient retention of primary amines on the diamond hydride column.

Table 33. Comparison of efficiency and asymmetry factor on the silica hydride and diamond hydride columns using UV detection.

Table 34. Comparison of efficiency and asymmetry factor on the silica hydride and diamond hydride columns using UV detection. 


\section{Introduction}

\section{A. Chromatography}

Chromatography is the most common technique to separate samples having closely related components in complex mixtures. In chromatography, the column holds the stationary phase surface of the packing material which is used to affect analyte attraction. The mobile phase which carries the samples is passed through the column. The components with higher affinity for the stationary phase will spend more time in the column; whereas the components with more affinity towards the mobile phase will elute along with it through the column. The difference in the retention time is the basis of separation of compounds in a mixture.

In 1906, Russian botanist Mikhail Tswett described several types of chromatography techniques for the first time [1]. A fundamental classification of chromatography techniques is mainly divided into three categories according to the nature of the stationary phase and the mobile phase: (1) liquid chromatography, (2) gas chromatography (GC), and (3) supercritical fluid chromatography. The mobile phases used in these techniques are solvents, gases, and supercritical fluids, respectively. Unlike gas chromatography, which can only be used for volatile samples, liquid chromatography can be used for a variety of volatile, non volatile, and ionic samples [1]. During the 1970s, most chemicals were separated using different techniques including paper 
chromatography, open-column chromatography, and thin layer chromatography. However, these techniques were not sufficient for resolution between similar compounds and quantification of compounds. During this time, to overcome these issues, high pressure liquid chromatography (HPLC) was developed to separate compounds at elevated pressure (up to 6000psi). Since then HPLC separations quickly improved with the development of different system components and column packing materials. In addition, different types of detectors and data handling devices improved the system to a high level of sensitivity and performance.

\section{B. Theory of High Performance Liquid Chromatography (HPLC)}

HPLC is one of the most widely used analytical techniques for the separation of various compounds such as amino acids, proteins, hydrocarbons, nucleic acids, carbohydrates, drugs, pesticides, metal-organic and non-organic substances. Liquid Chromatography was first invented in 1903 by a Russian botanist to separate pigments of green leaves on a chalk column. It was in the 1960s that more emphasis was placed on the development of liquid chromatography and today it has become a robust separation technique [1]. The basic HPLC instrumentation is composed of a pump, injector, column, detector and data acquisition system. Out of these, the column is the most important component since the separation takes place inside it. The separation technique 
involves mass transfer between the two major components of HPLC: the stationary phase and the mobile phase [1]. The liquid mobile phase transports the sample through the column containing the stationary phase. As the sample travels through the column, the interaction between the sample with the stationary phase and the mobile phase causes retention of the sample on the stationary phase. The separation of different compounds is achieved based on the affinity of each sample towards the stationary phase or the mobile phase, leading to different retention times of each sample in the column. Based on the nature of the stationary phase and separation process, HPLC can be classified into five different modes: ion-exchange chromatography, partition chromatography, size-exclusion chromatography, affinity chromatography and adsorption chromatography. Depending upon the relative polarities of the stationary phase and the mobile phase, partition chromatography is further classified into two types: (1) normal phase (NP) and (2) reversed phase (RP). In normal phase chromatography, the mobile phase is non-polar (e.g., hexane, heptane or ethyl acetate) and the stationary phase is strongly polar (e.g., bare silica, cyano or amino bonded phase). Thus, the polar compounds are retained longer compared to less polar compounds. In reversed phase chromatography, the stationary phase is non-polar (bonded alkyl groups such as C5, C8, C18, etc.) and the mobile phase is relatively polar (e.g., acetonitrile-water or methanol-water). This results in longer retention of non-polar compounds in the column [1]. Using these two modes of chromatographic separation, compounds 
having either polar or non polar properties in a mixture can be separated. There are two possible elution modes: isocratic and gradient. In isocratic elution, the composition of the mobile phase remains constant throughout the separation, whereas in the gradient elution the composition of the mobile is changed throughout the process. Separation of a complex mixture containing compounds with wide varying affinity for the stationary phase becomes difficult when the mobile phase concentration is kept constant during the HPLC run. Due to the constant concentration of the mobile phase, compounds with the least affinity for the stationary phase will come out too soon or may co-elute with the void volume. The compounds with strong affinity for the stationary phase remain much longer in the column, causing broad peaks. Gradient elution is another method of elution where the concentration of the mobile phase is varied at certain intervals causing elution of a complex mixture in a reasonable time and giving narrower peak width and thus better separation of analytes.

A gradient profile can be linear or step based on the mixture to be analyzed. In a linear gradient the percentage change of the organic solvent in the mobile phase is constant throughout the separation. In a gradient elution method, the composition of the mobile phase is chosen in such a manner that weakly retained analytes are eluted first and then the composition of the mobile phase is changed so that the compounds that are strongly retained in the column will elute [2]. In gradient elution, factors such as the proportion of the organic component in the mobile phase during elution time, the total gradient time chosen and the time 
required to re-equilibrate the HPLC system between each gradient process are very important to get reproducible retention times from one HPLC run to another. Both reversed phase separation and normal phase separation can employ a gradient method [2].

\section{Aqueous Normal Phase Chromatography (ANP)}

Recently, a new chromatographic technique has been developed called aqueous normal phase chromatography (ANP) [3]. In aqueous normal phase chromatography, both polar as well as non-polar analytes can be retained on the same column. This mode shows the same retention behavior as normal phase but has some water as part of the binary solvent system. Under ANP conditions, hydrophilic compounds are retained longer on hydride-based stationary phases with an increasing amount of the least polar component in the mobile phase, usually $60 \%$ or more [3]. For a solvent composed of acetonitrile and water, as the concentration of acetonitrile increases the analytes are retained longer in the stationary phase.

ANP can operate in reverse phase as well as in normal phase. This unique property of a dual mode of separation is possible by modifying a silica hydride based surface with a bonded phase prepared by silanization/hydrosilation procedure. Silica hydride surface allows separation of polar solutes such as acids and bases by varying the mobile phase composition 
from $100 \%$ water to $100 \%$ organic solvent. When a $100 \%$ aqueous mobile phase is used, the silica hydride surface can retain non-polar solutes whereas when a $100 \%$ organic mobile phase is used, it can retain polar solutes. Thus, the transition from reverse phase to aqueous normal phase can be achieved with less difficultly in a single isocratic or gradient run on the same HPLC column. Additives like formic acid, acetic acid, ammonium acetate and ammonium formate are used to get better peak shape. These additives control the $\mathrm{pH}$ and the ionic strength of the solvent which leads to ANP retention of polar acids and bases [4].

\section{Resolution, Selectivity and Number of Theoretical Plates}

A few important parameters, determining column efficiency, need to be considered for chromatographic separation of compounds in a mixture, such as selectivity (denoted by $\alpha$ ), capacity factor (denoted by k), resolution (denoted by $R s)$, and number of theoretical plates (N) of a HPLC column. Measuring the number theoretical plates is equivalent to measuring the column efficiency.

Column selectivity, also called the separation factor, can be defined as how well the column can resolve different compounds. In other words, it is a measure of the difference in retention between two compounds in a mixture. It depends on the mobile phase type and its composition. It also depends on the column packing material [5]. It is mathematically defined by Equation 1 . 


$$
\alpha=\frac{k_{2}}{k_{1}}
$$

where, $\mathrm{k}_{1}$ and $\mathrm{k}_{2}$ are the capacity factors of the two different compounds. The capacity factor is the measure of retention of a compound. It is mathematically given by Equation 2 .

$$
k=\frac{t r-t_{0}}{t_{0}}
$$

where, $t_{r}$ is the retention time of a compound, and $t_{0}$ is the void volume of the column.

Resolution $\left(R_{s}\right)$ is described by how well two compounds are separated from each other. It is mathematically defined by Equation 3.

$$
R s=\frac{2\left(t r_{2}-t r_{1}\right)}{w_{2}+w_{1}}
$$

where, $\operatorname{tr}_{2}>\operatorname{tr}_{1}$ and $w$ is the peak width of a compound in the chromatogram.

The next parameter is the number of theoretical plates, or column efficiency. These plates do not really exist in a column, but they are actually a theoretical model for understanding the concept, shown in Figure 1 [6]. Plates are series of theoretical "equilibrium stages" or "extraction steps." One plate corresponds to the length of the stationary phase required for one "equilibration" or "extraction step" of the solute between the stationary phase and mobile phase. The width of a band of solute is related to the distance the solute travels in the column. It is imagined that the chromatographic column is made up of large number $(\mathrm{N})$ of such plates. Chromatography columns with high numbers of 
theoretical plates produce very narrow peaks, resulting in better separation. The analytes move down the column by the transfer of the equilibrated mobile phase from one theoretical plate to the next [5].

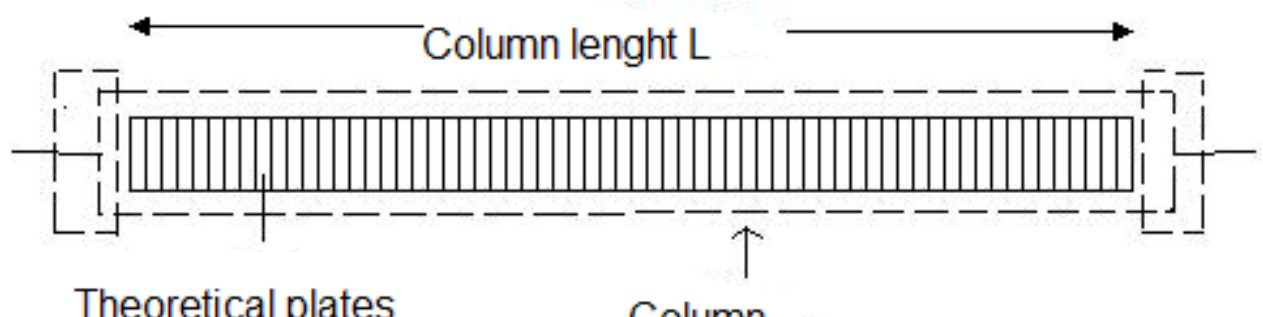

Figure 1. Concept of theoretical plates of a column.

This description introduces to another parameter called height equivalent to a theoretical plate (HETP), mathematically given by Equation 4. A lower HETP value of a column means higher column efficiency.

$$
H E T P=\frac{L}{N}
$$

where, $\mathrm{L}$ is the column length and $\mathrm{N}$ is the number of theoretical plates.

The column efficiency $\mathrm{N}$ is a measure of residence time distribution of a compound passing through the column. It is mathematically defined by Equation 5.

$$
N=16\left(\frac{t r}{w}\right)^{2}
$$


Resolution is a function of the three factors discussed previously: retention $\mathrm{k}$, selectivity $\alpha$, and efficiency $\mathrm{N}$. All these parameters are interdependent. Improving one parameter often degrades one or more of the others, so compromise becomes necessary to give an optimal HPLC solution for chromatographic separation of compounds. For a chromatographic separation of two peaks, the resolution is mathematically described by Equation 6 and Equation 7 for peak 1 and peak 2, respectively.

$$
\begin{aligned}
& R s=\frac{\sqrt{N_{1}}}{4}(\alpha-1)\left(\frac{k_{1}}{1+k_{1}}\right) \\
& R s=\frac{\sqrt{N_{2}}}{4} \frac{(\alpha-1)}{\alpha}\left(\frac{k_{2}}{1+k_{2}}\right)
\end{aligned}
$$

where $N_{1}, K_{1}$ are the efficiency and retention of peak 1 and $N_{2}, K_{2}$ are efficiency and retention of peak 2 .

When partially separated peaks are obtained, increasing $k$ can separate two overlapping peaks with longer retention and broader peaks. Whereas decreasing $k$ can make two compounds co-elute at a very short retention time. Using different mobile phase compositions can result in different $k$ values. However, there is a potential disadvantage when increasing $k$ to separate compounds in a sample because the separation may take too long so that strongly retained compounds may not elute [5]. 


\section{E. Silica Surface: Surface Modification}

There are many available solid supports that can be used for HPLC column packing after surface modification. Examples of such solid supports are titania, alumina, zirconia, thoria, and silica. Of them, silica is the most favored due to its commercial availability [1]. Silica possesses some desirable properties like high mechanical strength under pressure, fabrication of particles with specific diameter, pore size and surface area with high accuracy, and wide $\mathrm{pH}$ operating range of 2.00-7.50 [7]. Initially, silica had irregular shaped particles with a wide pore size distribution resulting in poor reproducibility and limited use in combination with various normal phase solvents. The popularity of RP separations resulted from significant improvement in column chromatography by modification of silica through bonding hydrophobic moieties using organosilane technology. The development of spherical shape silica particles of $10 \mu \mathrm{m}$ to even smaller size added to the versatility of HPLC for enhanced efficiency and resolving power or column resolution. Silica technology kept improving by providing high purity materials that minimized the presence of trace metals like aluminum. On the silica hydride surface, $\mathrm{Si}-\mathrm{OH}$ groups are replaced with $\mathrm{Si}-\mathrm{H}$ groups. This reduces the interaction of free residual silanols with solute ions. This new and more pure material was called Type-B silica. The other important parameter is the pore size since it determines the area of interacting surface of the stationary phase. In general, larger pore silica is useful for the analysis of 
macromolecules, since it provides sufficient room for interaction, whereas smaller pore silica is useful for the analysis of small molecules $[8,9]$.

Amorphous silica with a porous structure has three kinds of structural silanols attached to its surface: isolated, geminal and vicinal. These are hydrophilic in nature [11]. The silica surface also contains exposed siloxane bonds (Si-O-Si). Silanol groups are strong adsorption sites whereas siloxane sites are usually hydrophobic in nature which makes it suitable for non-polar analyte retention when the mobile phase has a higher ratio of aqueous component. Out of the two, silanols are more interesting to chromatographers since it can be used to bond other species [10]. The silica surface can be best described by Figure 2 .

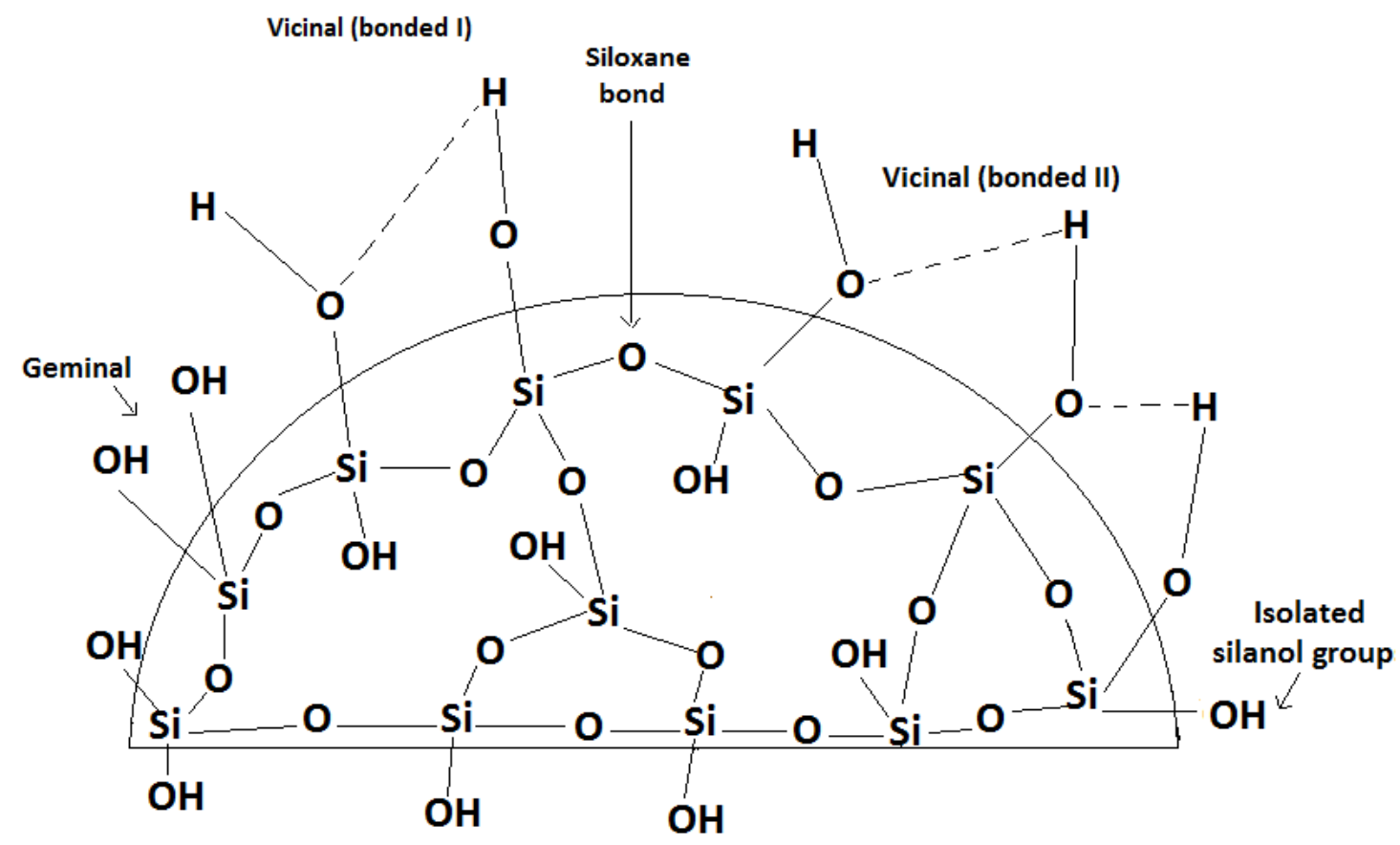

Figure 2. Unmodified silica surface. 
The concentration of silanol $(\mathrm{SiOH})$ groups on the silica surface is estimated anywhere between $7-8 \mu \mathrm{mol} / \mathrm{m}^{2}$. Depending on the type of analysis, the presence of these active groups can be advantageous or disadvantageous. But there is a consensus that out all of the active groups free silanols are the most significant since they are widely used for silica surface modification by attaching organic moieties. Figure 3 shows the active groups found on a silica surface [12].

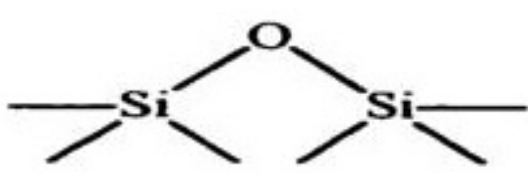

Siloxane

$\mathrm{H}$

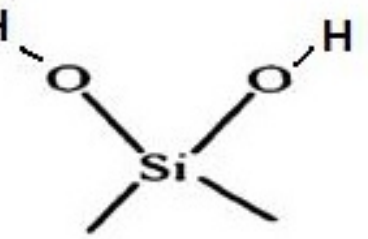

Geminal Silanols

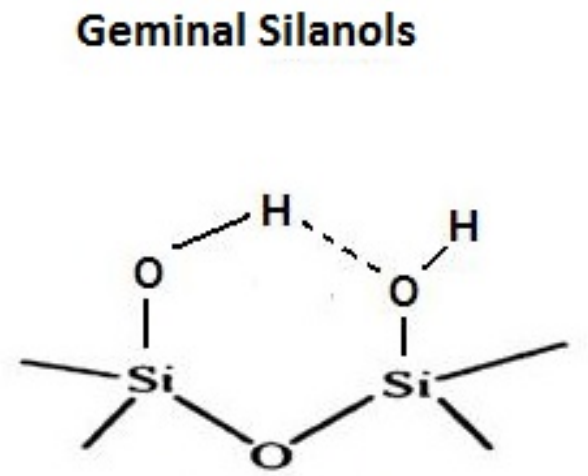

Vicinal Silanols

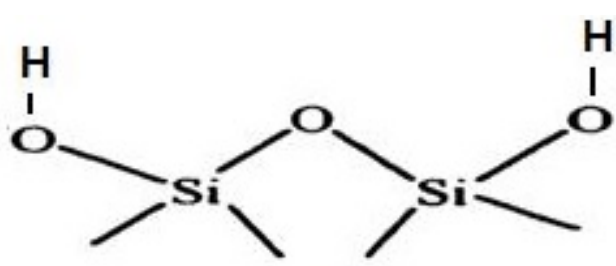

Free Silanols

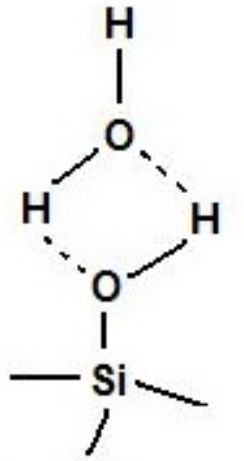

Bound water

Figure 3. Active groups found on the silica surface. 
Silica supports have been shown to be superior to alumina and zirconia in terms the mechanical stability, ease of fabrication, efficiency and performance. But silica has some disadvantages, like $\mathrm{pH}$ stability beyond a certain range, severe peak tailing with basic analytes and irreproducibility of results with the same chemistry columns. To overcome these problems, chemical modification of the surface of silica has been used. The field of modification is a well researched field of surface chemistry where a number of conversion methods to attach bonded organic moieties to silanol groups and their effects have been studied. These reactions are esterification, organosilanization, chlorination followed by reaction with Grignard reagents, TES silanization and hydrosilation [13]. In all these methods, the organic moieties are covalently attached to the silica surface.

Esterification was the earliest method developed and it involves a reaction between a silanol group and an alcohol to form a Si-O-C linkage [11]. This linkage is however not very stable. This reaction forms a silicon-oxygencarbon linkage between silica surface and organic moiety with a simple coupling reaction. The resulting bonded phase is not stable with aqueous mobile phases [13]. The next method is referred to as organosilanization. In this reaction, an organosilane is covalently bonded to the silica surface. This method is widely used for synthesizing commercial HPLC columns. This reaction can be used to form a monomeric phase or a polymeric phase. A polymeric phase is formed when three reactive groups are used which leads to cross-linking between the adjacent organosilane groups. Both reactions result in a Si-O-Si-C 
linkage but their properties are different. The monomeric phase goes through hydrolysis under moderately acidic and moderately basic conditions. But it has good chromatographic efficiency and it is a reproducible method. Whereas, a polymeric phase gives better hydrolytic stability but the reaction is not reproducible compared to the monomeric phase $[5,14]$. The main reason behind the hydrolytic instability is the production of an acid by-product during the reaction. In the third method, the free silanols on the silica surface are chlorinated with a compound like thionyl chloride to form an unstable intermediate. Next, the intermediate compound is then reacted with an organometallic compound using a Grignard reagent or organolithium compound resulting in Si-C linkage. This $\mathrm{Si}-\mathrm{C}$ linkage is hydrolytically stable. But this method also has two disadvantages: (1) It is very sensitive to moisture during the chlorination and reduction step, and (2) formation of a metallic salt by-product, which is difficult to wash off and can act as a potential contaminant on the silica surface $[14,15]$. Because of these disadvantages the above method is still considered cumbersome and complicated for a direct Si-C linkage formation. The various methods of silica surface modifications are shown in Figure 4. 


\begin{tabular}{|c|c|c|}
\hline $\begin{array}{l}\text { REACTION } \\
\text { NAME }\end{array}$ & REACTION & $\begin{array}{c}\text { SURFACE } \\
\text { LINKAGES } \\
\text { FORMED }\end{array}$ \\
\hline $\begin{array}{l}\text { ESTERI- } \\
\text { FICATION }\end{array}$ & $\equiv \mathrm{Si}-\mathrm{H}+\mathrm{R}-\mathrm{OH} \longrightarrow \equiv \mathrm{Si}-\mathrm{OR}+\mathrm{H}_{2} \mathrm{O}$ & $\mathrm{Si}-\mathrm{O}-\mathrm{C}$ \\
\hline $\begin{array}{l}\text { ORGANO- } \\
\text { SILANIZATION }\end{array}$ & $\begin{array}{l}\equiv \mathrm{Si}-\mathrm{OX}+\mathrm{X}-\mathrm{SiR}_{2}{ }_{2} \mathrm{R} \longrightarrow \mathrm{Si}-\mathrm{O}-\mathrm{SiR}_{2}{ }_{2} \mathrm{R}+\mathrm{HX} \\
\text { where X-halide and R-alkyl } \\
\equiv \mathrm{Si}-\mathrm{OH}+\mathrm{X}_{3}-\mathrm{Si}-\mathrm{R} \rightarrow \equiv \mathrm{Si}-\mathrm{O}-\mathrm{Si}-\mathrm{R}+\mathrm{HX}\end{array}$ & $\begin{array}{l}\mathrm{Si}-\mathrm{O}-\mathrm{Si}- \\
\mathrm{C}\end{array}$ \\
\hline $\begin{array}{l}\text { CHLORINATION } \\
\text { FOLLOWED BY } \\
\text { GRIGNARD'S } \\
\text { REAGENT OR } \\
\text { AN ORGANO- } \\
\text { LITHIUM } \\
\text { COMPOUND }\end{array}$ & $\begin{array}{l}\equiv \mathrm{Si}-\mathrm{OH}+\mathrm{SOCL}_{2} \longrightarrow \equiv \mathrm{Si}-\mathrm{Cl}+\mathrm{SO}_{2}+\mathrm{HCl} \\
\quad \equiv \mathrm{Si}-\mathrm{Cl}+\mathrm{RMgBr} \rightarrow \equiv \mathrm{Si}-\mathrm{R}+\mathrm{MgClBr} \\
\mathrm{OR} \\
\quad \equiv \mathrm{Si}-\mathrm{Cl}+\mathrm{Li}-\mathrm{R} \rightarrow \equiv \mathrm{Si}-\mathrm{R}+\mathrm{Li}-\mathrm{Cl}\end{array}$ & $\mathrm{Si}-\mathrm{C}$ \\
\hline $\begin{array}{l}\text { TES- } \\
\text { SILANIZATION }\end{array}$ & 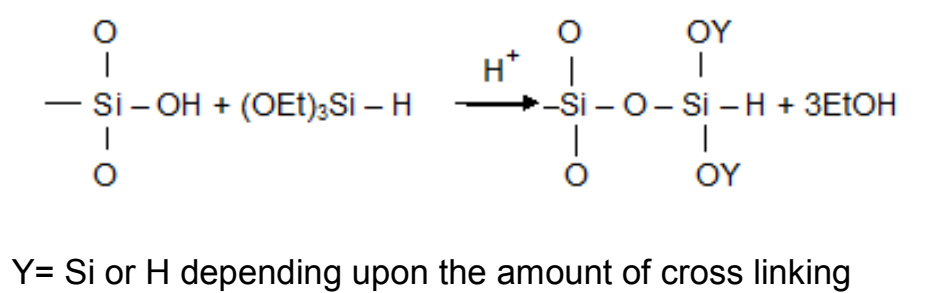 & $\begin{array}{l}\mathrm{Si}-\mathrm{H} \\
\text { monolayer }\end{array}$ \\
\hline $\begin{array}{l}\text { HYDRO- } \\
\text { SILATION }\end{array}$ & Cat. = catalyst, metal complex such as hexachloroplatinic & $\mathrm{Si}-\mathrm{C}$ \\
\hline
\end{tabular}

Figure 4. Various methods of silica surface modification. 
A more recent approach involves silanization followed by a hydrosilation reaction. This method was first introduced by Pesek et al., [14]. The main reason behind this approach is to develop a uniform silica surface with active sites for further modification by terminal olefins. In the process of silanization, the product of hydrolysis of triethoxysilane (TES) or other related silanes forms a Si-H monolayer on the silica substrate. This method is less time consuming and much simpler than the chlorination method. The stable hydride monolayer formed by silanization can withstand aqueous conditions for a longer period of time and it converts more than $95 \%$ of silanols to $\mathrm{Si}-\mathrm{H}$. The attachment of an organic group at the silica hydride surface to form a Si-C bond occurs via a hydrosilation reaction [14].

\section{F. Advantages of Using Hydride-based Stationary Phases}

Silica hydride based columns possess high mechanical strength, ease of fabrication and high column efficiency. With silica derivatization it is possible to manufacture bonded phases using a variety of compounds having different functional groups. The main reaction sites on an ordinary silica surface are the silanol groups. The modification of the silica matrix can be analyzed using magicangle-spinning (MAS) ${ }^{29} \mathrm{Si}$ NMR and diffuse reflectance infrared Fourier transform spectroscopy (DRIFT) [16]. Even after modification there are still many silanol groups which remain. These groups interact with analytes causing poor resolution and peak tailing. Other impurities like metals such as $\mathrm{Fe}, \mathrm{Al}$ and $\mathrm{Zn}$ 
can also cause peak tailing and alter retention properties during HPLC runs. Being highly acidic and the presence of very low amount of metal impurities makes silica a better choice as starting material for stationary phase synthesis.

The comparison of the chemical composition between silica and silica hydride is shown in Figure 5. On the silica hydride surface, Si-OH groups are replaced with $\mathrm{Si}-\mathrm{H}$ groups. This key difference between the two materials leads to different surface properties and thus a significant difference in their chromatographic properties. Silanol groups are highly polar in nature whereas the $\mathrm{Si}-\mathrm{H}$ groups are weakly hydrophobic. Many investigations have proved that silica hydride columns can be employed for various HPLC separations.
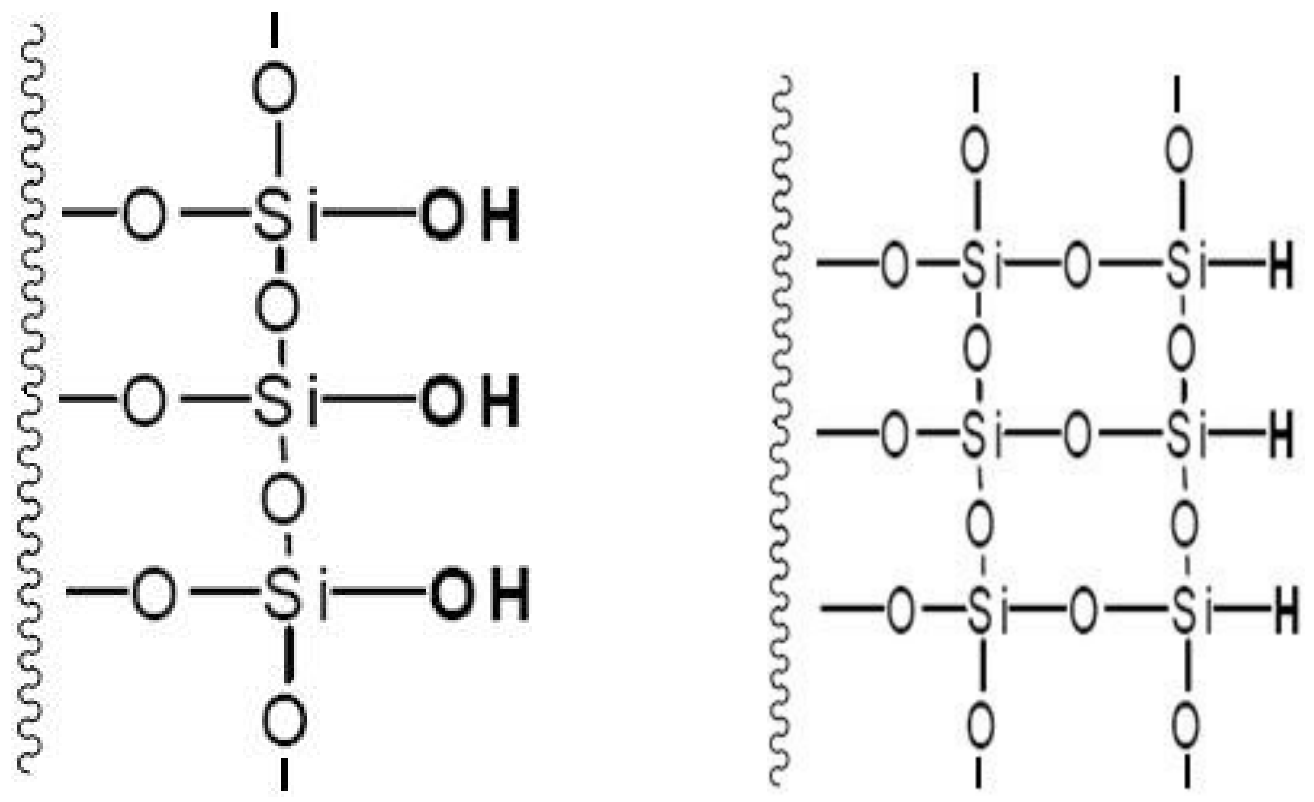

Figure 5. Ideal structures of a native silica surface (left) and silica hydride surface (right). 


\section{G. High Performance Liquid Chromatography (HPLC)}

The HPLC process starts with the introduction of small amount of sample into a stream of solvent (the mobile phase) that flows through a column (the stationary phase) containing a bed of packing material like silica beads. The sample is then carried through the column by a continuous flow of the mobile phase, which is delivered by a precise pumping system. As the separated compounds leave the column, they are sensed by an appropriate detector. The detector then transmits a signal to a recorder, and the separation is documented in the form of a sequence of peaks called a chromatogram. Each discrete peak may represent a particular compound. A schematic of an HPLC system is shown in Figure 6. Besides the stationary phase and mobile phase, the detector is also an important part of an HPLC system. There are different kinds of detectors such as UV, fluorescence, refractive index, evaporative light scattering, and mass spectrometers. The choice of a detector depends on the nature of the sample and the purpose behind the analysis because some detectors are destructive in nature. A mass spectrometer detector is the most sensitive of all and considered a universal detector. It also furnishes molecular information about the sample. It is a very expensive detector, which is a drawback $[2,19]$. The fluorescence detector requires the presence of a fluorophore in the sample to be detected. Samples that do not have these radiation-absorbing moieties attached to them need to be derivatized before they can be detected. The refractive index detector is considered a universal system as it outputs a signal based on the changes in 
the refractive index of the mobile phase composition. The main disadvantages of this type of detector are the inability to use it under gradient elution conditions and poor sensitivity $[2,19]$.

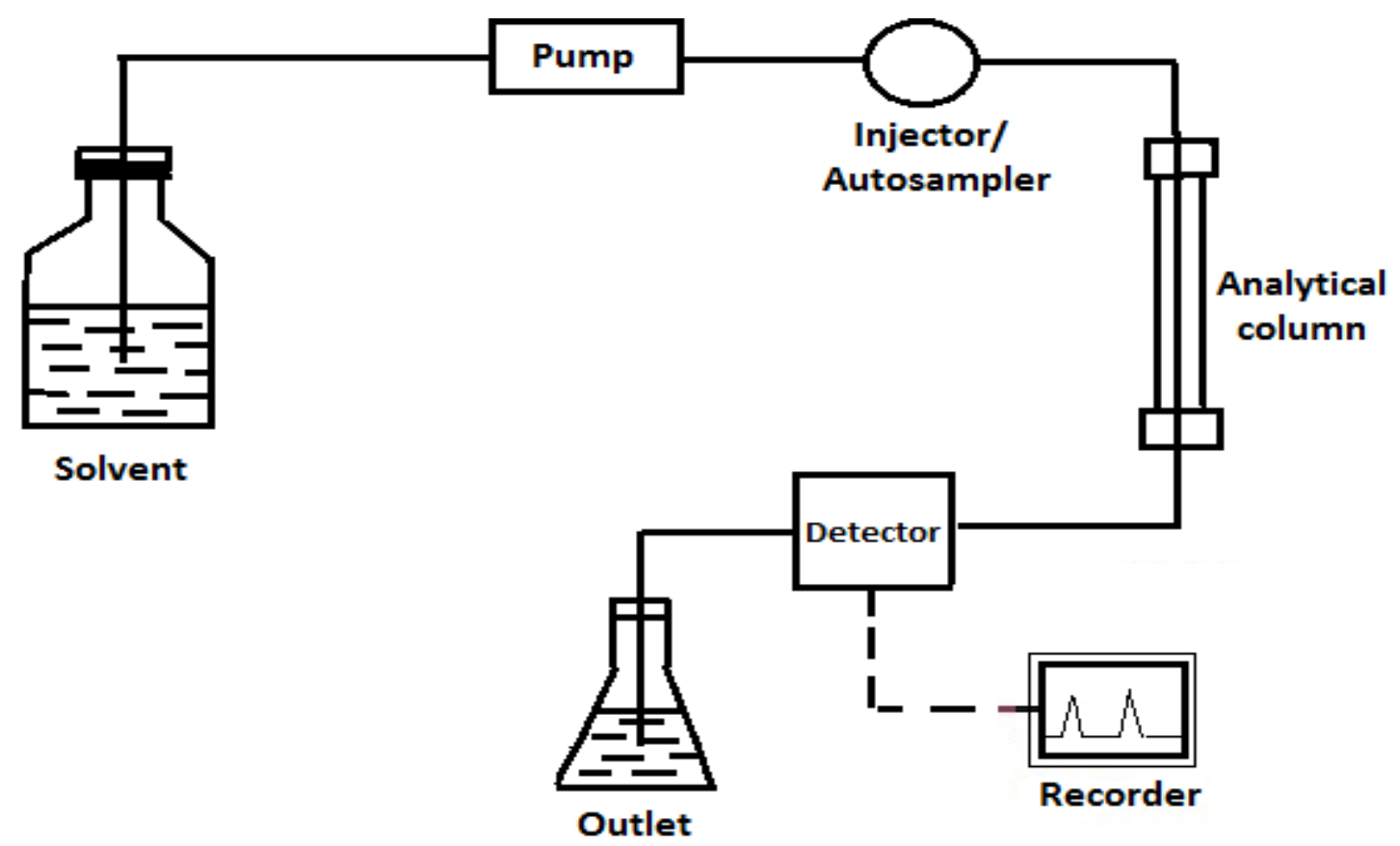

Figure 6. Schematic diagram of a HPLC system.

\section{Background of Detectors Used}

\section{$1.1 \quad$ Ultraviolet (UV) Detector}

UV detectors are the most widely used non-destructive detectors. They work on the principle of Beer's law which states that the absorbance of an analyte at a particular UV wavelength is directly proportional to its concentration. 
Sophisticated UV detectors have the ability to scan multiple UV wavelengths by use of a filter, prism, or grating [2]. However, it is important to note that the analyte needs to absorb an appropriate wavelength for UV detectors to work. Analytes that do not absorb in the UV region require chromophores to absorb the radiation. Samples that do not have these radiation-absorbing moieties attached to them are required to be derivatized before they can be detected $[1,19]$.

\subsection{Evaporative Light Scattering Detector (ELSD)}

During this research, two detectors were used: a evaporative light scattering detector (ELSD) and a UV detector. An evaporative light scattering detector has reasonably good sensitivity. It can be used with gradient elution and does not require sample derivatization.

The relationship between concentration of the sample and peak area is non-linear because the amount of light scattering is a function of the particle size and not the concentration of the sample. A schematic diagram of an ELSD detector is shown in Figure 7. ELSD operation is based on three simple steps: nebulization, evaporation, and detection [5]. In the nebulization step, the mobile

phase is converted into small droplets, typically by use of nitrogen. As the aerosol formed after nebulization travels through a heated chamber, it evaporates the more volatile mobile phase and leaves only a smaller cloud of solute particles [20]. 


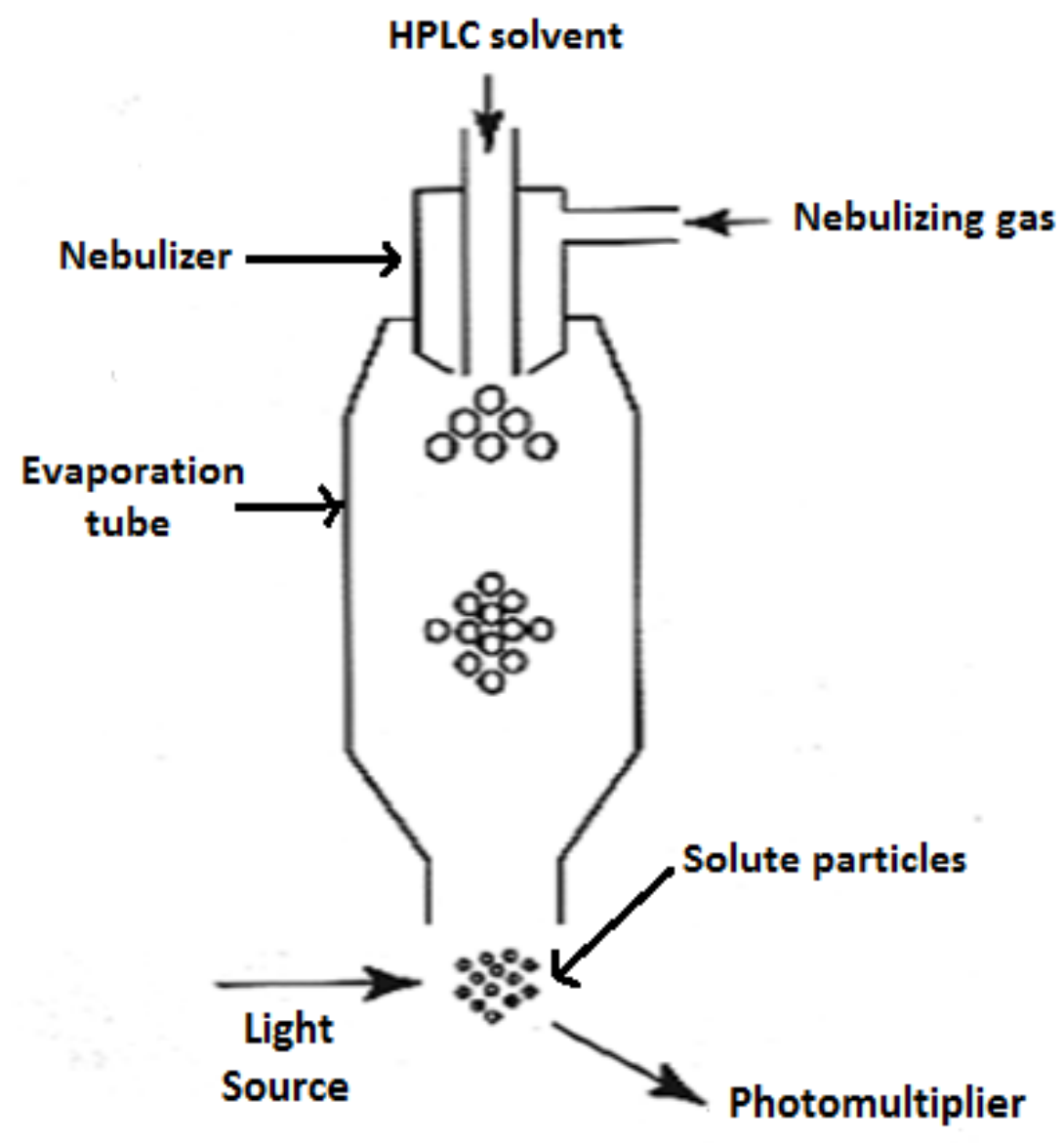

Figure 7. ELSD detector operation.

Typical challenges for ELSD detectors are higher flow rates and mobile phases which are not easily evaporated. SofTA Thermo-Split technology from SofTA Corporation is a recent development in ELSD detector technology [20]. Here the aerosol splitter combines a bend in the spray chamber along with temperature controlled walls. When the mobile phase is easy to evaporate, the walls are heated so that it evaporates the mobile phase, leaving only the smaller particles that easily pass through the bend. When the mobile phase is difficult to evaporate, or when the flow rates are high, the walls are cooled [20]. Thus when 
the aerosol travels it condenses into large particles with higher momentum that collide with the wall and exit down the drain. Using this technology, it is very easy to achieve a split ratio of $99 \%-1 \%$; that too is controlled in a smooth fashion by changing the temperature of the spray chamber in $1^{\circ} \mathrm{C}$ steps from $0^{\circ} \mathrm{C}$ to $80^{\circ} \mathrm{C}$. The detector utilized in this research, the SofTA model 400 ELSD, uses this patented SofTA thermo-split technology [20]. A schematic representation of this technology is shown in Figure 8. Figure 8A depicts the cooled walls, and 8B shows the heated walls.

The evaporative light scattering detector (ELSD) is known as a universal detector because it can detect any analyte as long as it is less volatile than the mobile phase. Compared to a UV detector, it does not require chromophores nor does it require the analyte to be UV active [1]. It also does not require sample preparation or sample derivatization. No solvent front peaks are observed, and it gives a stable gradient profile. It gives a stable baseline even with a rapidly changing gradient. The detector is not sensitive to surrounding temperature changes. In contrast to an MS detector, it is less complex and has lower operating costs. However, there are drawbacks too. It is a destructive detector and cannot be used for quantitative measurements because the concentration is not proportional to peak area. This is due to the fact that the detector works on the principle of light scattering, which depends on the particle size rather than analyte concentration. It has high sensitivity and can detect analytes of very small concentration typically in the nanogram range. But this detector requires 
that the mobile phase be more volatile than the analyte so it cannot detect analytes with high volatility. If a non-volatile mobile phase is used it will lead only to detector noise, which is considered as a drawback of this detector $[2,5,20]$.
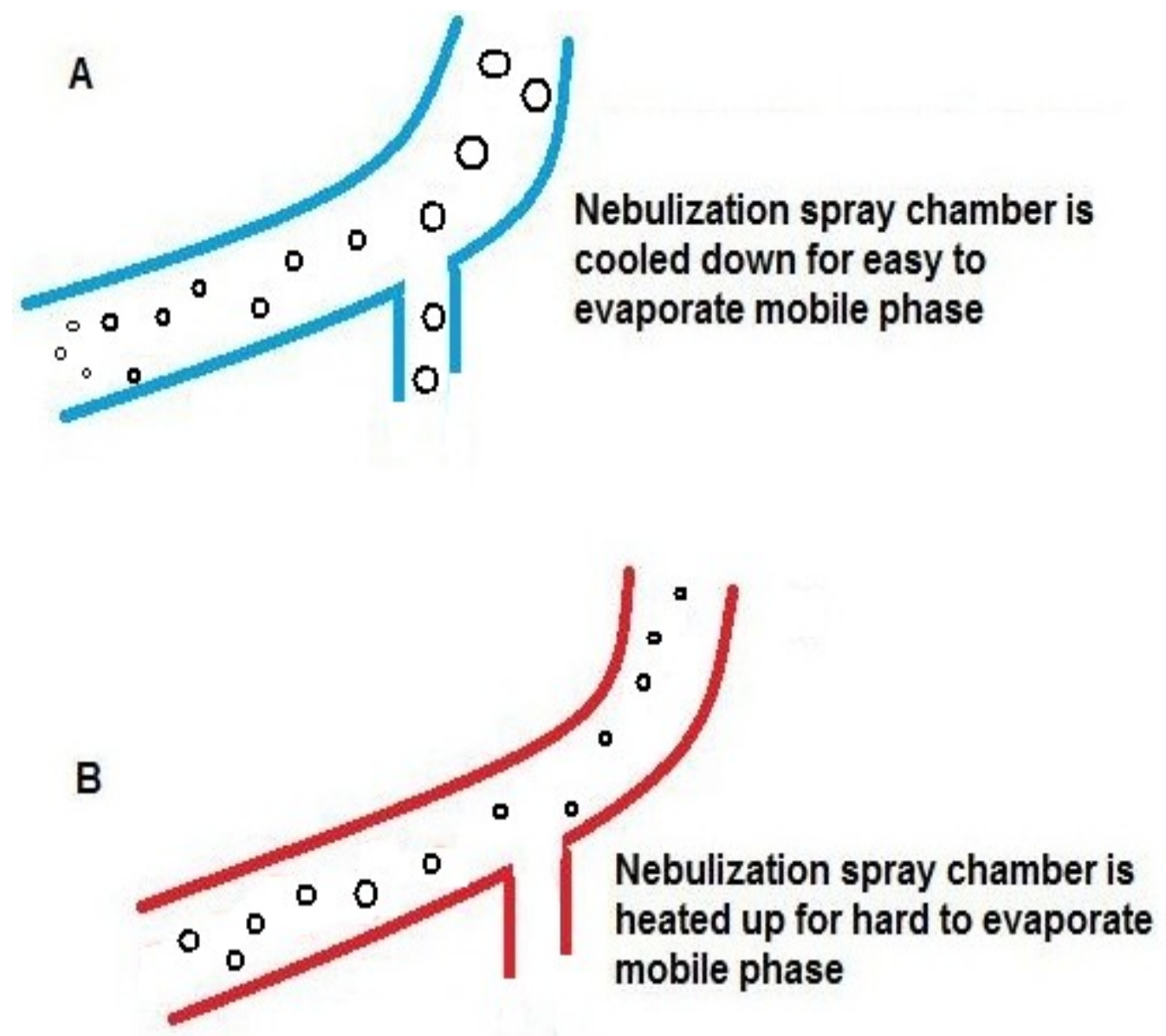

Figure 8. A schematic diagram of thermo-split technology. A. Cooled surface. B. Heated surface. 


\section{H. Types of Samples Used in the Evaluation of HPLC Columns}

In this research, small polar molecules including amino acids, nucleosides, nucleotides, ergogenic acids and primary amines were studied for their affinity towards silica hydride and diamond hydride based columns. The first group tested was amino acids. Amino acids are building blocks of peptides and proteins. Amino acids have a typical pH between 6 and 8 . They exist in nature as zwitterions, with positive and negative charge within a molecule. Amino acids contain amine and carboxylic acid functional groups. The hydrophobicity of the amino acid molecules comes from the polarity of its side chain. A protein consists of several hydrophilic and hydrophobic amino acids that determine its tertiary structure. This tertiary structure determines its interaction with the stationary phase [2].

Often a molecule cannot be differentiated from other compounds in the sample without changing its properties through derivatization. In general, amino acids have this basic problem, which requires their derivatization to be detected with standard detectors, except for tryptophan, tyrosine and phenylalanine that absorb in the UV range. Therefore in this research there was no derivatization done for L-tryptophan. The ability of ANP to separate both polar and non polar molecules makes amino acids a good choice of solute to study.

The second classes of compounds used in this research are nucleosides and nucleobases. They form the main part of DNA and RNA. Previously they have been reported to be separated by reversed phase chromatography method 
but this mode gives very low retention time [21]. The other mode used is lonexchange chromatography, which requires use of buffers and salts in the mobile phase that are not suitable for an MS detector. Hence, nucleoside and nucleobase become a good choice of solutes to be investigated with ANP chromatography.

The next group of compounds used was ergogenic acids like creatinine. Creatinine is obtained after the break down of creatine to produce energy. It is a popular nutritional supplement used by athletes. Creatinine is a compound that is made primarily in the liver and then transported to muscles where it is used as an energy source. The functionality of the human kidney can be evaluated by checking levels of creatinine in human urine. This biological relevance makes creatinine an important choice of solute for study $[22,23]$.

The last group of compounds used in this study consists of primary amines. Amines are organic derivatives of ammonia. They are found in many pharmaceutical compounds, used as initiators for polymer reactions, $\mathrm{pH}$ buffers and surfactants. They are classified as primary, secondary or tertiary depending on the number of alkyl or aromatic groups attached to the nitrogen [24]. This research attempts to separate primary amines under ANP isocratic and gradient conditions using the silica hydride and diamond hydride columns. 


\section{Silica Hydride and Diamond Hydride Stationary Phases}

Silica hydride and diamond hydride stationary phases used in this research were previously fabricated by Dr. Pesek's group. The silica hydride was packed in a $75 \times 4.6 \mathrm{~mm}$ i.d. column and the diamond hydride was also packed in a $75 \times 4.6 \mathrm{~mm}$ i.d column.

Out of many varieties of silica hydride stationary phases, the diamond hydride is the one which is unique. The diamond hydride surface is prepared by chemically bonding a small amount of carbon (approximately $2 \%$ ) to the silica hydride surface. The attached carbon gives hydrophobic properties to the stationary phase in addition to the ANP retention properties possessed by silica hydride stationary phases. Thus the diamond hydride stationary phase can perform both ANP and reversed phase mechanisms. These hydrophobic properties can be used in the reversed phase retention mechanism. Diamond hydride columns have been used in many research studies to separate hydrophobic and hydrophilic compounds using different mobile phase compositions. The mobile phase chosen consists of a higher percentage of organic solvent like acetonitrile or acetone and a small percentage of water for ANP retention. Diamond hydride has been shown to be an excellent choice for amino acids analysis, small organic acids and carbohydrates using LCMS $[25,26]$. 


\section{J. Research Goals}

The primary goal of this research was to analyze the separation ability of the silica hydride column in the aqueous normal phase mode for small polar molecules. Compounds like amino acids, primary amines, nucleosides, ergogenic acids and nucleobases were tested to investigate their retention time on the silica hydride column. Part of the research work was carried out using a ELSD detector, but the majority of the work was done using a UV detector due to the further unavailability of a ELSD detector. Retention maps of individual compounds were plotted under isocratic conditions, and their ANP behavior was demonstrated for the silica hydride column.

The second objective was to evaluate the ANP behavior of the diamond hydride stationary phase using the same analytes, instruments, and same conditions. The third goal was to compare the silica hydride stationary phase with the diamond hydride stationary phase. The retention and separation of analytes on both columns (silica hydride and diamond hydride) were studied and compared to analyze whether the small amount of carbon on the diamond hydride surface contributed to the retention and separation of the compounds. 


\section{Experimental}

\section{A. Materials}

The analytical columns used in this research were silica hydride $(75 \times 4.6$ $\mathrm{mm}$ i.d.) and diamond hydride $(75 \times 4.6 \mathrm{~mm}$ i.d.). Both columns were previously fabricated by Dr. Pesek's group. The particle size of both stationary phase materials was $4.2 \mu \mathrm{m}$, and the pore size was $100 \AA$. Acetonitrile and water, which was obtained from a Mili-Q Purification system (Millipore Co., Bedford, MA), were used as the mobile phase.

All the compounds were stored in the freezer at $0^{\circ} \mathrm{C}$ before use. Before making a sample solution, the additive (listed in Table 1) was placed in DI water. The solute was added to this solution. Finally, acetonitrile was added into the solution. Sample solutions were prepared in the range of $0.5 \mathrm{mg} / \mathrm{mL}$ to $1 \mathrm{mg} / \mathrm{mL}$.

\subsection{Compounds Used for the Characterization of Columns}

The compounds used for this research in the preparation of the samples and mobile phase are listed in Table 1 and the compounds used for the characterization of stationary phases are listed in Table 2. 
Table 1. Compounds used in the mobile phase and for the preparation of the samples.

\begin{tabular}{|c|c|}
\hline Chemical Name & Manufacturing company \\
\hline Acetonitrile & Fisher Chemicals Fair Lawn, NJ \\
\hline Formic acid & Spectrum Mfg. corp. Gardena, CA \\
\hline Hydrochloric acid & Fisher Chemicals Fair Lawn, NJ \\
\hline
\end{tabular}

Table 2. Compounds used for the evaluation of columns.

\begin{tabular}{|c|c|}
\hline Chemical Name & Manufacturing company \\
\hline L-Tryptophan & Sigma Chemical Co. St Louis, MO \\
\hline Adenosine & CALBIOCHEM Co. LA \\
\hline Creatinine & CALBIOCHEM Co. LA \\
\hline Guanine & $\begin{array}{c}\text { NBC National Biochemical Corp. } \\
\text { Twinsburg, } \mathrm{OH}\end{array}$ \\
\hline Guanidine (Hydrochloride) & Sigma Chemical Co. St Louis, MO \\
\hline 2-Phenethylamine & $\begin{array}{l}\text { Matheson Coleman \& Bell, Cincinnati, } \\
\qquad \mathrm{OH}\end{array}$ \\
\hline Benzylamine & Sigma Chemical Co. Milwaukee, WI \\
\hline 2,6-Diaminopurine & Sigma Chemical Co. Milwaukee, WI \\
\hline $\begin{array}{l}\text { 1,2,4,5-Benzenetetramine- } \\
\text { tetrahydrochloride }\end{array}$ & Sigma Chemical Co. Milwaukee, WI \\
\hline 4-Chloro-1,2-phenylenediamine & Sigma Chemical Co. Milwaukee, WI \\
\hline 1,2,4,5-Benzenetetracarboxamide & Sigma Chemical Co. Milwaukee, WI \\
\hline 3,4-Diaminobenzophenone & Sigma Chemical Co. Milwaukee, WI \\
\hline
\end{tabular}




\subsection{Structures of Compounds Used for Chromatographic Characterization}

Figure 9 shows the structures of each compound used for the chromatographic characterization.

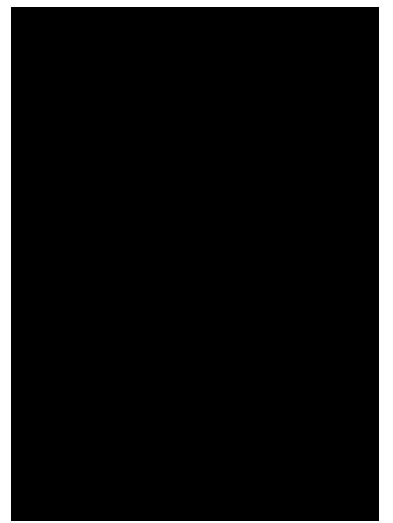

Guanidine

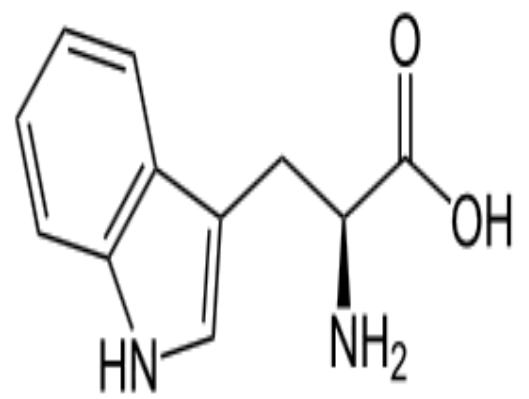

L-Tryptophan<smiles>Nc1ncnc2c1ncn2[C@@H]1O[C@H](CO)[C@@H](O)[C@H]1O</smiles>

Adenosine

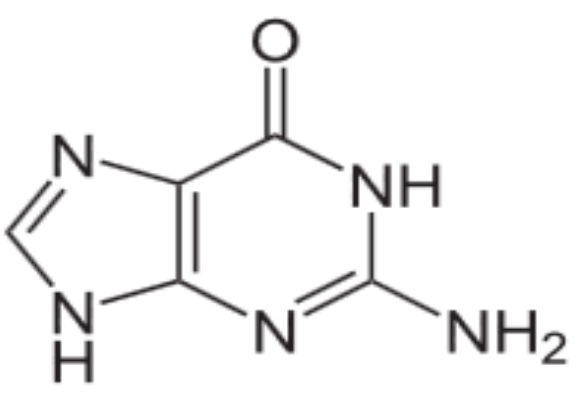

Guanine 


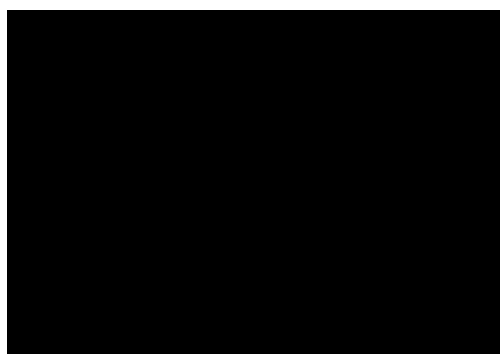

Creatinine

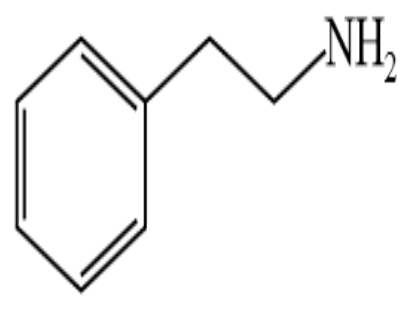

2-Phenethylamine

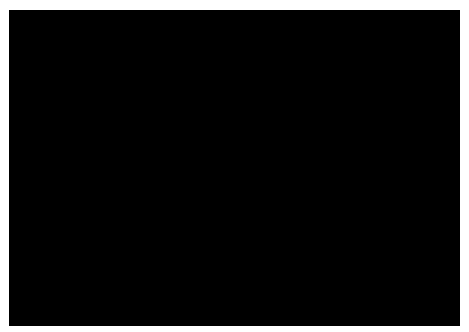

2,6-Diaminopurine

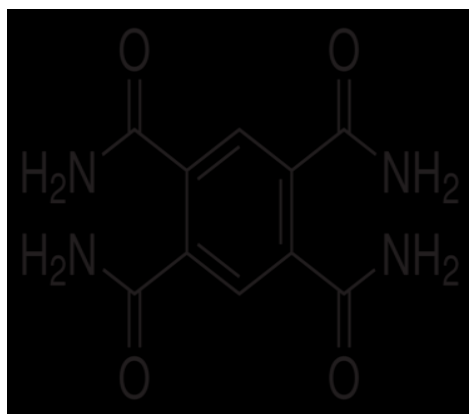

1,2,4,5-Benzenetetracarboxamide

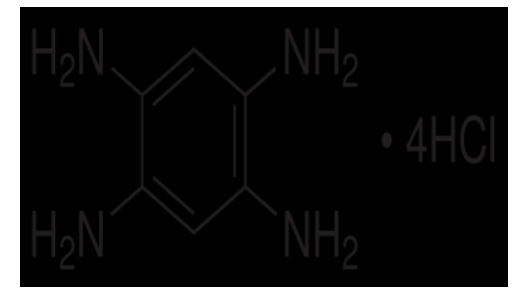

1,2,4,5-Benzenetetramine tetrahydrochloride

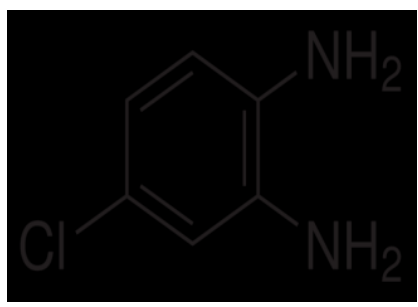

4-Chloro-1,2-phenylenediamine

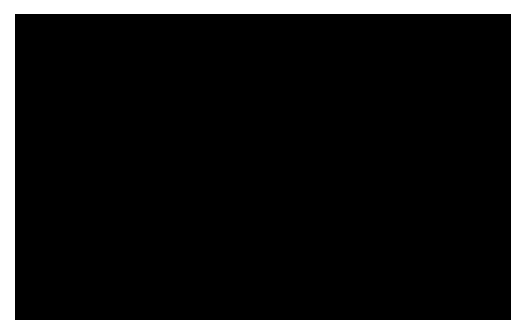

Benzylamine

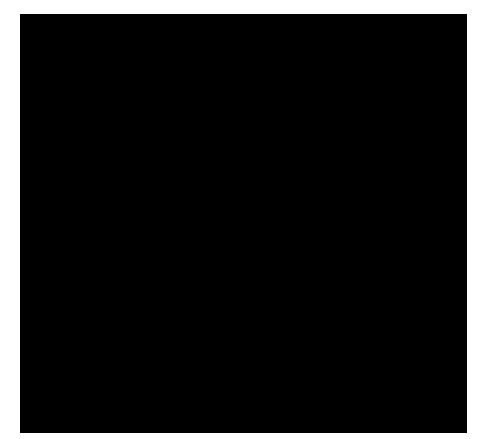

3,4-Diaminobenzophenone

Figure 9. Structures of compounds used for chromatographic characterization. 


\section{B. Instrumentation and Operation Procedure}

Two types of detectors and thus two different types of HPLC systems were employed in this research. The first HPLC system included a SoftA Corporation Model 400 evaporative light scattering detector (ELSD), a Rheodyne Model 7126 manual injector with a $20 \mu \mathrm{L}$ injection loop, a Waters in-line degasser and a LDC Milton Roy CM400 multiple solvent delivery system. The light scattering and detection process was carried out by a $670 \mathrm{~nm}$ wavelength laser diode with hermetically sealed photodiode. For the nebulization of the column eluent, it was required to maintain a continuous supply of nitrogen gas at a constant pressure of 65 psi with an approximately 2.5 SLMP gas consumption rate. The data acquisition and storage was done by Chemstation Software, a HP 35900C multichannel interface and an HP laserjet 2100M printer was used for making hard copies of the chromatograms. All the mobile phases were vacuum filtered using a $0.2 \mu \mathrm{m}$ Nylon 66 membrane (Alltech Associates, Inc. Deerfield, IL). A common mobile phase flow rate of $0.5 \mathrm{~mL} / \mathrm{min}$ was applied to all HPLC separations. The deionized water used in the HPLC separations was prepared using a Milli- $\mathrm{Q}^{\mathrm{TM}}$ purification system (Millipore Corp., Bedford, MA).

A spectrophotometric detector, Shimadzu SPD-6A, was used with the same HPLC system in this research for samples which absorb UV. The other components of the system remained the same. The mobile phase flow rate was also unchanged from $0.5 \mathrm{~mL} / \mathrm{min}$. 
The other HPLC system used in this research was an HP series 1050 instrument equipped with a variable wavelength detector, a quaternary pump, autosampler and an in-line degasser. The software used for data analysis was Chemstation HP 1050. The pump flow rate was set at $0.5 \mathrm{~mL} / \mathrm{min}$. The volume of the samples for each injection was $5 \mu \mathrm{L}$ and the column temperature was maintained at ambient. 


\section{Results and Discussion}

The main objective of the research was to characterize the silica hydride and diamond hydride based stationary phases. The dimensions of silica hydride and diamond hydride columns were $7.5 \mathrm{~cm} \times 4.6 \mathrm{~mm}$. Both column types demonstrated ANP behavior when employed for HPLC separation of various analyte types. Two different detectors (UV and ELSD) were used for this research. Both columns were conditioned for half an hour using 50:50 water and methanol before any HPLC run. Each HPLC run was repeated twice to check if the HPLC results were repeatable. The retention performances of these columns are discussed in the following sections.

\section{A. Chromatographic Evaluation of HPLC Columns}

The goal of the research was to characterize the silica hydride and diamond hydride based columns. Both columns demonstrated aqueous normal phase behavior. Amino acids, nucleobases, nucleosides, ergogenic acids and primary amines were tested for chromatographic characterization of the silica hydride column. The results obtained by using the $\mathrm{Si}-\mathrm{H}$ column were then compared with the diamond hydride column.

Two different detectors, ELSD and UV were used for chromatographic characterization of silica hydride and diamond hydride columns. The parameters used for HPLC runs with the ELSD detector were a flow rate of $0.5 \mathrm{~mL} / \mathrm{min}$, the column temperature was ambient and the injection volume was $20 \mu \mathrm{L}$. The 
composition of the mobile phase was 50:50 water/acetonitrile (both premixed with $0.1 \%$ formic acid) and the concentration of samples was $1 \mathrm{mg} / \mathrm{mL}$. These parameters were optimized as per an isocratic system. To get a smooth baseline, the temperature of the drift tube and spray chamber were adjusted. The temperature of the drift tube was elevated to $65^{\circ} \mathrm{C}$ to evaporate the mobile phase whereas the temperature of the spray chamber was kept at room temperature $\left(25^{\circ} \mathrm{C}\right)$. The $0.1 \%$ formic acid (FA) added to the mobile phase evaporated during light scattering detection. At the start of each day, a water blank was run to remove any contamination from the previous injection. The main goal of this part of research was to characterize and establish ANP behavior of the silica hydride stationary phase.

\section{ANP Behavior of Si-H Column Using an ELSD Detector}

\subsection{ANP Behavior of Adenosine}

Adenosine was dissolved in 50:50 acetonitrile /water (both premixed with $0.1 \%$ formic acid) at a concentration of $1 \mathrm{mg} / \mathrm{mL}$. Adenosine was analyzed under isocratic conditions using an ELSD detector to study its retention characteristics on the silica hydride column. A water blank was run before each HPLC run to remove any contamination from the previous run. The ELSD parameters were adjusted and stabilized according to the initial conditions of the isocratic run. The mobile phase flow rate was set at $0.5 \mathrm{~mL} / \mathrm{min}$ with a $20 \mu \mathrm{L}$ injection volume at 
ambient temperature. Figure 10 represents the chromatogram of adenosine on the silica hydride column at $80: 20$ acetonitrile/water with $0.1 \%$ formic acid mobile phase composition.

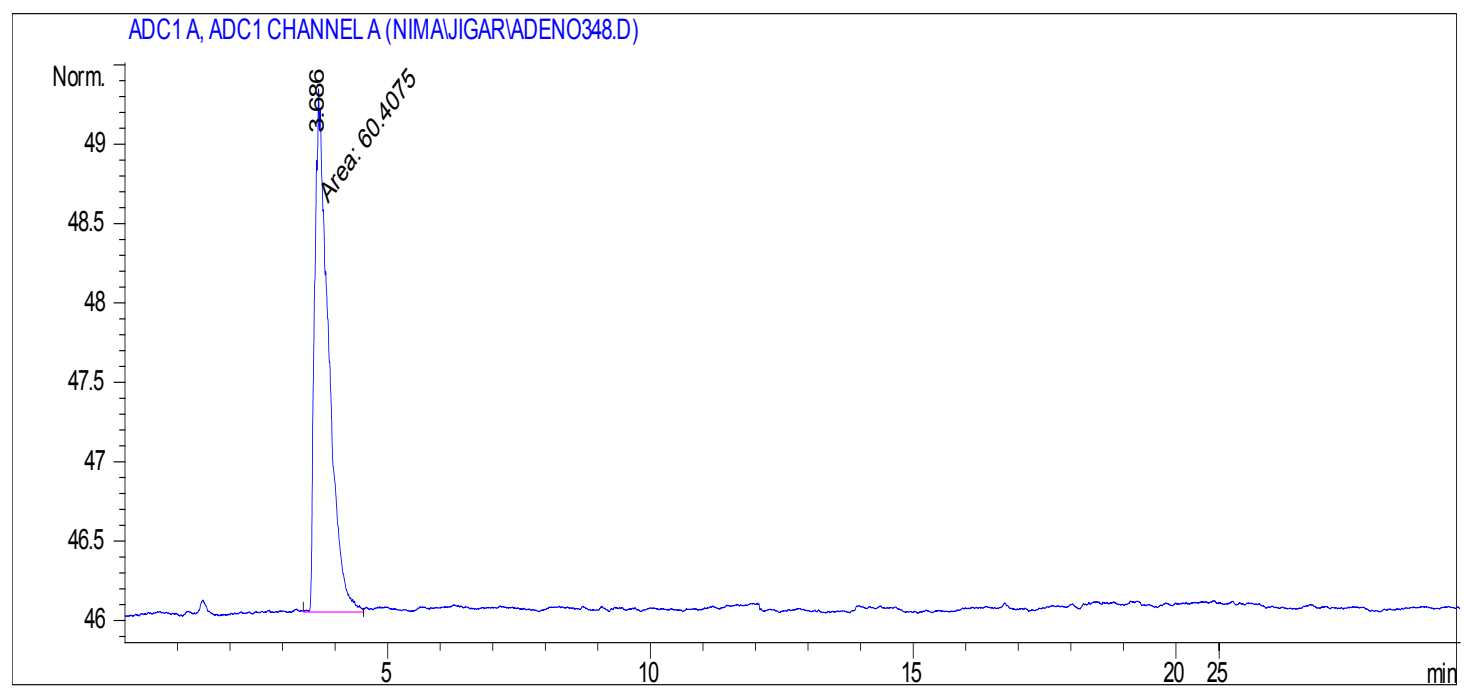

Figure 10. Chromatogram of adenosine on the silica hydride stationary phase at $80: 20$ acetonitrile/water mobile phase (both premixed with $0.1 \%$ formic acid).

An evaporative light scattering detector was used for the analysis. Table 3 shows the retention times at various percentage concentrations of mobile phase compositions. As the concentration of acetonitrile was increased from $50 \%$ to $90 \%$, the retention time of adenosine increased gradually, confirming the ANP behavior of this compound. Figure 11 represents the characteristic ANP retention map of adenosine. More work was done for adenosine in the isocratic mode using a UV detector and will be discussed in a subsequent section. 
Table 3. Retention time of adenosine on the silica hydride column using ELSD.

\begin{tabular}{|c|c|c|}
\hline \multicolumn{2}{|c|}{ Concentration (\%) } & Retention time(min) \\
\hline Acetonitrile with 0.1\% FA & $\begin{array}{c}\text { DI water with } \\
\text { 0.1\% FA }\end{array}$ & Adenosine \\
\hline 50 & 50 & 2.33 \\
\hline 60 & 40 & 2.74 \\
\hline 70 & 30 & 2.89 \\
\hline 80 & 20 & 3.69 \\
\hline 90 & 10 & 4.74 \\
\hline
\end{tabular}

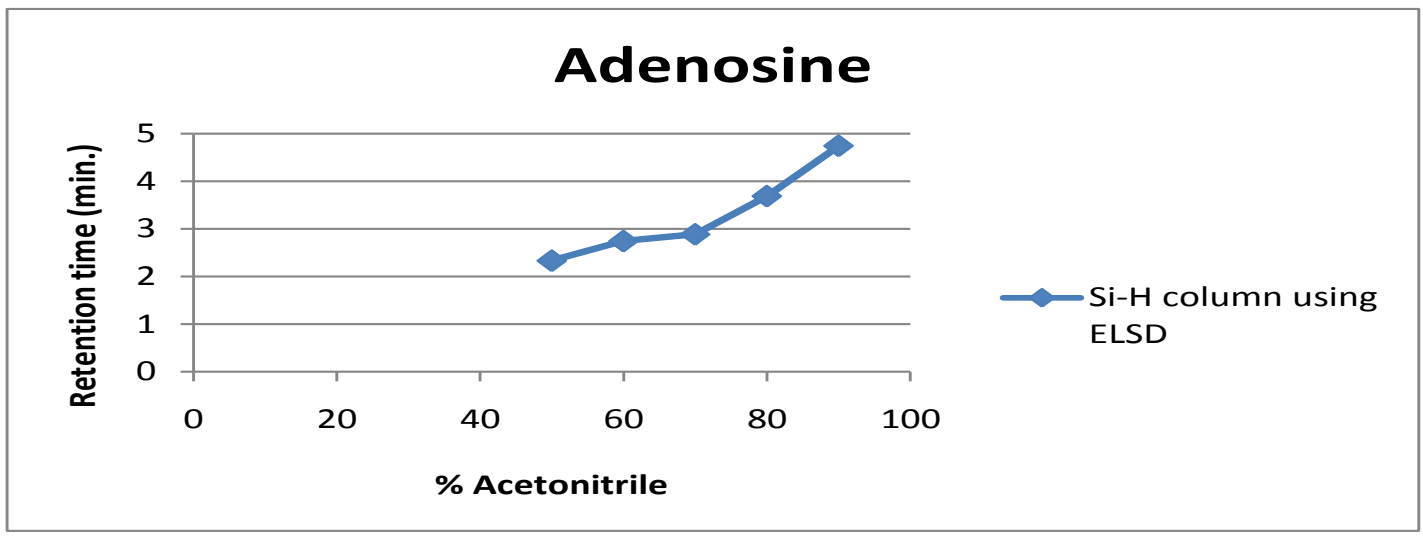

Figure 11. Retention map of adenosine on the silica hydride column using ELSD.

\subsection{ANP Behavior of Guanine}

Guanine was difficult to dissolve so $200 \mu \mathrm{L} \mathrm{HCl}$ solution was mixed with 50:50 acetonitrile/water (both premixed with $0.1 \%$ formic acid) to prepare a 1 $\mathrm{mg} / \mathrm{mL}$ sample solution. The ELSD parameters were adjusted and stabilized according to the initial conditions of the isocratic test. The mobile phase flow rate for HPLC was set at $0.5 \mathrm{~mL} / \mathrm{min}$ with a $20 \mu \mathrm{L}$ injection volume. Guanine was 
analyzed using aqueous normal phase conditions in the isocratic mode on the silica hydride column. Figure 12 shows the chromatograms of guanine for $70: 30$ and $80: 20$ acetonitrile/water (with $0.1 \%$ formic acid) concentrations.
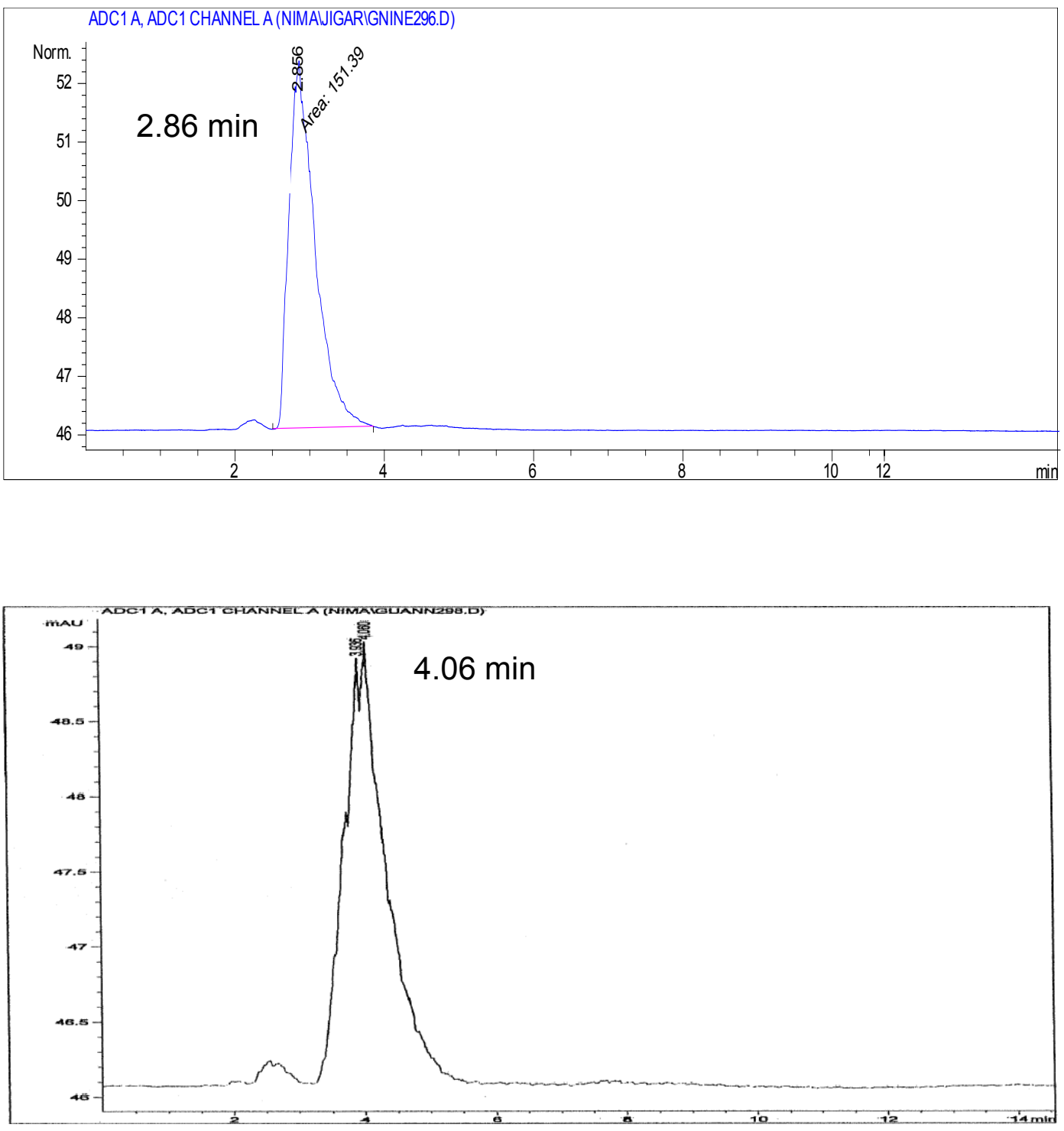

Figure 12. Chromatograms of guanine obtained using the silica hydride stationary phase 70:30 acetonitrile/water (Top) and 80:20 acetonitrile/water (Bottom) mobile phase (both premixed with $0.1 \%$ formic acid). 
Table 4 shows the retention times at various $\%$ concentrations of mobile phase compositions. As the concentration of acetonitrile was increased from $50 \%$ to $90 \%$, the retention time of guanine increased gradually and confirmed the ANP behavior of this compound. Figure 13 represents the ANP retention map of guanine as the amount of acetonitrile increases.

Table 4. Retention time of guanine on the silica hydride column using ELSD.

\begin{tabular}{|c|c|c|}
\hline \multicolumn{2}{|c|}{ Concentration (\%) } & Retention time(min) \\
\hline Acetonitrile with $\mathbf{0 . 1 \%}$ FA & DI water with 0.1\% FA & Guanine \\
\hline 50 & 50 & 1.97 \\
\hline 60 & 40 & 2.16 \\
\hline 70 & 30 & 2.86 \\
\hline 80 & 20 & 4.06 \\
\hline 90 & 10 & 4.48 \\
\hline
\end{tabular}

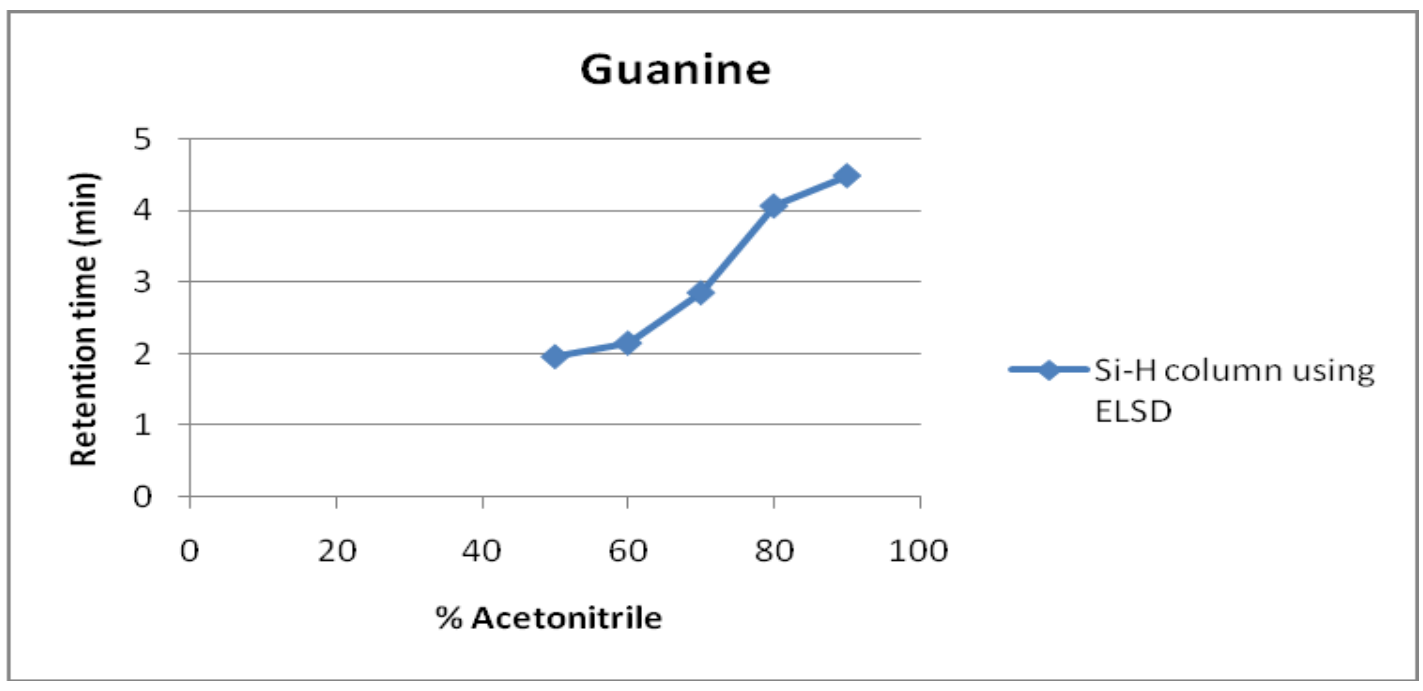

Figure 13. Retention map of guanine on the silica hydride using ELSD. 


\subsection{ANP Behavior of L-Tryptophan}

L-Tryptophan was analyzed under ANP isocratic conditions on the silica hydride column. The mobile phase used for the analysis consisted of acetonitrile/ water with $0.1 \%$ formic acid. The concentration of L-tryptophan was $1 \mathrm{mg} / \mathrm{mL}$ in a 50:50 acetonitrile/ water (both premixed with $0.1 \%$ formic acid) solution. The mobile phase flow rate was set at $0.5 \mathrm{~mL} / \mathrm{min}$ with an injection volume of $20 \mu \mathrm{L}$. The ELSD parameters were adjusted and stabilized according to the initial conditions of the isocratic test. Different compositions of mobile phase were used for the analysis starting from 50:50 acetonitrile/water (both premixed with $0.1 \%$ formic acid) to $80: 20$ acetonitrile/water (both premixed with $0.1 \%$ formic acid). Figure 14 shows the chromatogram of L-tryptophan for 50:50 mobile phase composition.

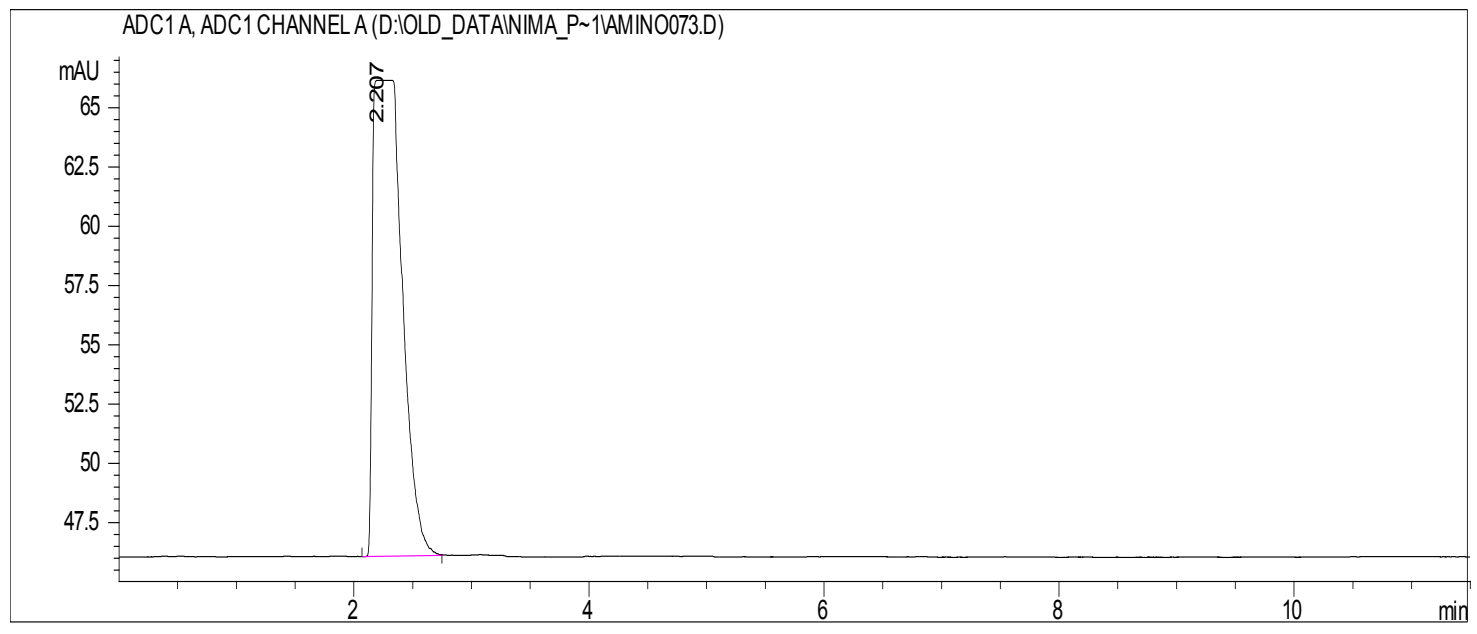

Figure 14. Chromatogram of L-tryptophan on the silica hydride stationary phase at 50:50 acetonitrile/water mobile phase (both premixed with $0.1 \%$ formic acid). 
Table 5 lists the retention times and Figure 15 shows the corresponding characteristic retention map of L-tryptophan observed on the silica hydride column at various $\%$ concentrations of mobile phase compositions. As the concentration of acetonitrile was increased from $50 \%$ to $80 \%$, the retention time of L-tryptophan also increased, which confirmed the ANP behavior of this compound.

Table 5. Retention time of L-tryptophan on the silica hydride using ELSD.

\begin{tabular}{|c|c|c|}
\hline \multicolumn{2}{|c|}{ Concentration (\%) } & Retention time(min) \\
\hline $\begin{array}{c}\text { Acetonitrile with } \\
\mathbf{0 . 1 \%} \text { FA }\end{array}$ & DI water with 0.1\% FA & L-Tryptophan \\
\hline 50 & 50 & 2.21 \\
\hline 60 & 40 & 2.51 \\
\hline 70 & 30 & 3.07 \\
\hline 80 & 20 & 5.01 \\
\hline
\end{tabular}

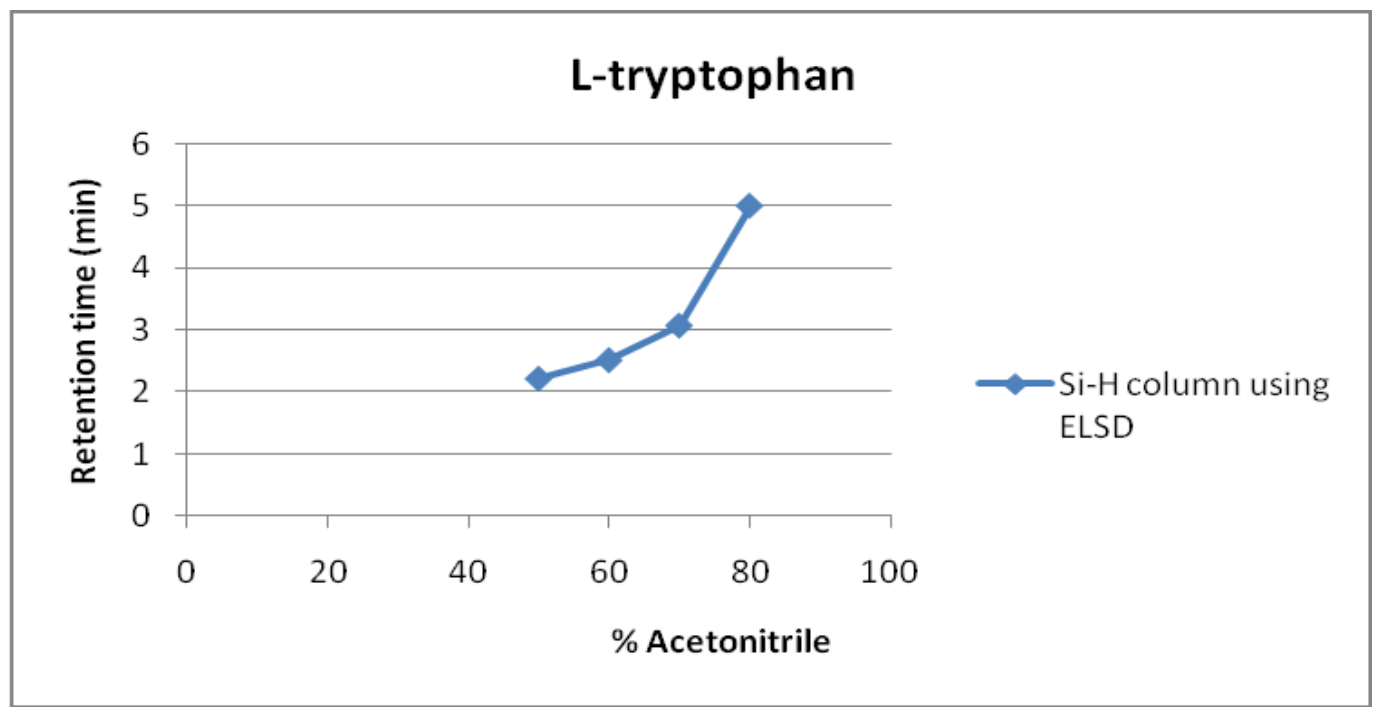

Figure 15. Retention map of L-tryptophan on the silica hydride using ELSD. 


\subsection{ANP Behavior of Guanidine}

The concentration of guanidine used for this analysis was $1 \mathrm{mg} / \mathrm{mL}$.

Guanidine was analyzed in the ANP isocratic mode on the silica hydride column. An ELSD detector was used for the analysis. The mobile phase used was acetonitrile/water (both premixed with $0.1 \%$ formic acid) with a $0.5 \mathrm{~mL} / \mathrm{min}$ flow rate and a $20 \mu \mathrm{L}$ injection volume. The ELSD parameters were stabilized and set according to the starting conditions of isocratic test.

As shown in Table 6, guanidine showed a moderate ANP retention with increasing acetonitrile concentration from $50 \%$ to $80 \%$. Figure 16 shows the ANP retention map of guanidine.

Table 6. Retention time of guanidine on the silica hydride using ELSD.

\begin{tabular}{|c|c|c|}
\hline \multicolumn{2}{|c|}{ Concentration (\%) } & Retention time(min) \\
\hline Acetonitrile with 0.1\% FA & DI water with 0.1\% FA & Guanidine \\
\hline 50 & 50 & 2.42 \\
\hline 60 & 40 & 2.89 \\
\hline 70 & 30 & 3.72 \\
\hline 80 & 20 & 4.44 \\
\hline
\end{tabular}




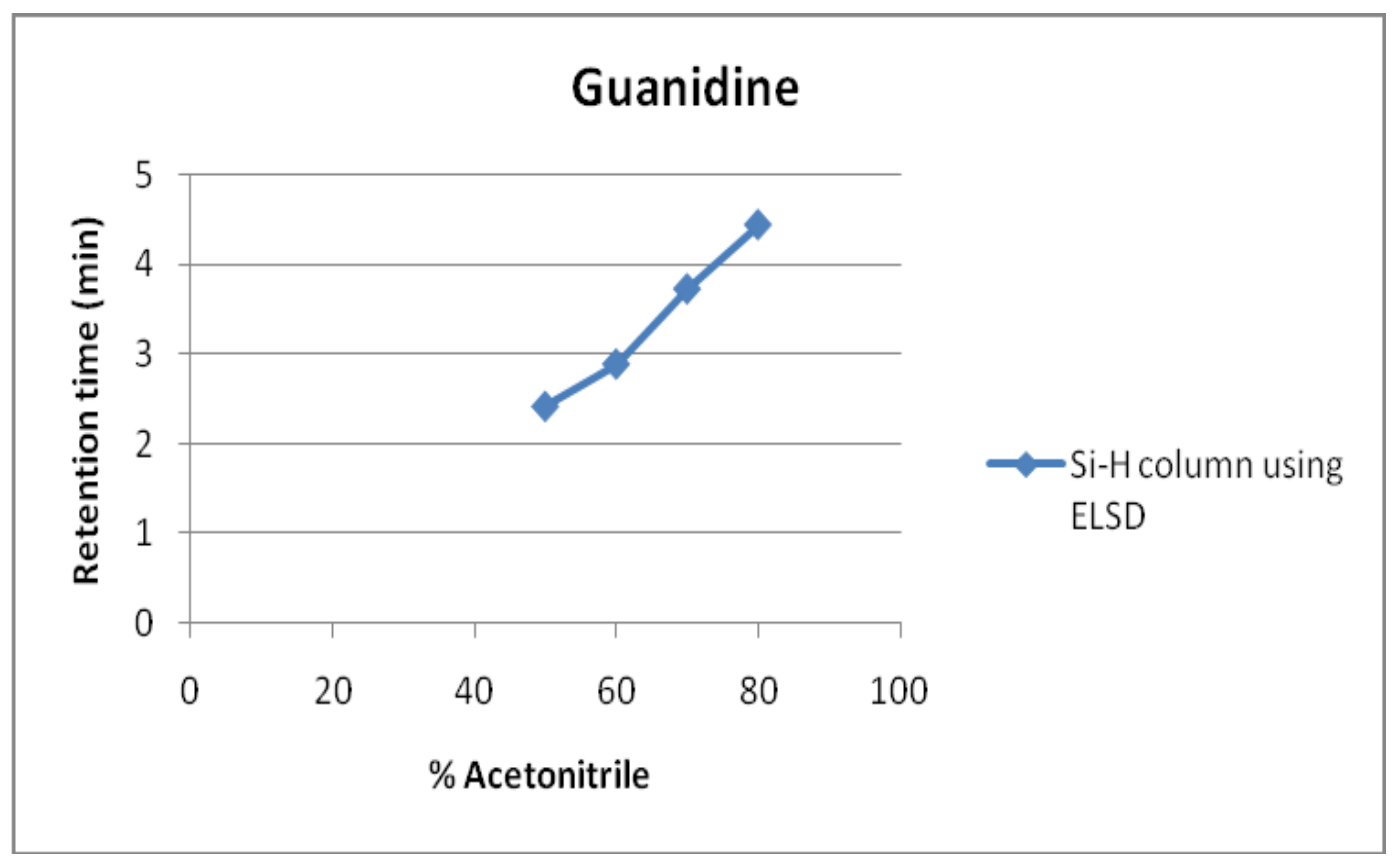

Figure 16. Retention map of guanidine on the silica hydride column.

\subsection{ANP Behavior of Creatinine}

The concentration used for the ANP behavior of creatinine was $1 \mathrm{mg} / \mathrm{mL}$.

Creatinine was analyzed in the ANP isocratic mode on the silica hydride column. An ELSD detector was used for the analysis. The mobile phase used was acetonitrile/water (both premixed with $0.1 \%$ formic acid). The flow rate was set at $0.5 \mathrm{~mL} / \mathrm{min}$ with a $20 \mu \mathrm{L}$ injection volume. The ELSD parameters were stabilized and set according to the initial conditions of the isocratic test. As shown in Table 7, the polar compound creatinine can be retained using the aqueous normal phase elution mode. It shows the ANP retention of creatinine with increasing acetonitrile concentration from $50 \%$ to $90 \%$ on the silica hydride column. 
Table 7. Retention time of creatinine on the silica hydride column using ELSD.

\begin{tabular}{|c|c|c|}
\hline \multicolumn{2}{|c|}{ Concentration (\%) } & Retention time(min) \\
\hline Acetonitrile with 0.1\% FA & DI water with 0.1\% FA & Creatinine \\
\hline 50 & 50 & 2.51 \\
\hline 60 & 40 & 2.92 \\
\hline 70 & 30 & 3.82 \\
\hline 80 & 20 & 5.93 \\
\hline 90 & 10 & 6.11 \\
\hline
\end{tabular}

The retention map of creatinine is plotted in Figure 17. As shown in the figure, creatinine displayed good ANP retention as the concentration of acetonitrile increased from $50 \%$ to $90 \%$, the retention times also increased. This data obtained indicates the typical ANP behavior of creatinine on the silica hydride column.

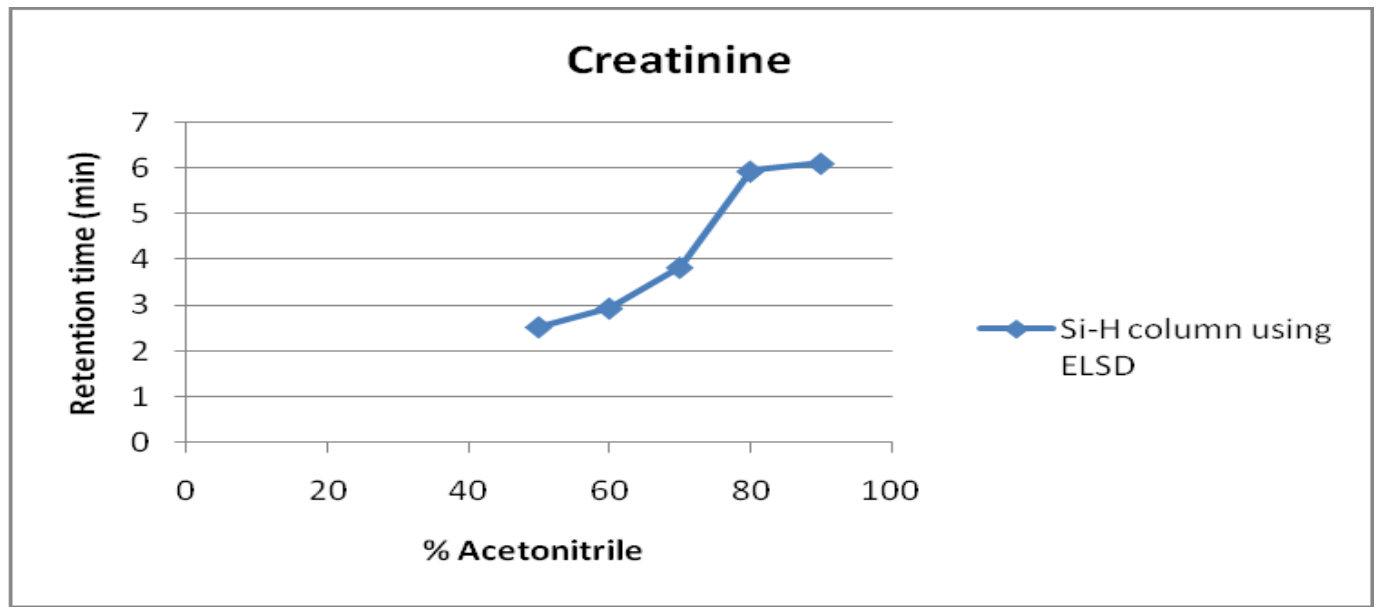

Figure 17. Retention map of creatinine on the silica hydride column using ELSD. 
The same compounds were also analyzed using a UV detector and the results are discussed in a later section. Considerably more work has been done using UV detection in comparison to ELSD.

\section{ANP Behavior of Si-H Column Using UV Detector}

Because of the further unavailability of the ELSD detector the emphasis of HPLC separations for the remaining of the research was shifted from the ELSD detector to the UV detector. All further ANP retention studies of the silica hydride and diamond hydride columns were performed using a UV detector. Different classes of compounds e.g. amino acids, primary amines, nucleobases, nucleosides and ergogenic acids were analyzed to establish their characteristic ANP retention. Primary amines were tested under isocratic as well as gradient conditions. The characterization of the each column type was carried out in the aqueous normal phase mode. The parameters used for HPLC with UV detection were a flow rate $0.5 \mathrm{~mL} / \mathrm{min}$, an injection volume $5 \mu \mathrm{L}$, and the column temperature was ambient. The concentration of the samples was $0.5 \mathrm{mg} / \mathrm{mL}$ in 50:50 water/acetonitrile (both premixed with $0.1 \%$ formic acid) solution. The columns were maintained at the starting conditions of the gradient or isocratic system until a stable baseline was obtained. A water blank was run to eliminate any contamination from the previous measurements. The gradient programs used for this research are shown in Table 8 and Table 9. 
The goal of this research was to perform chromatographic characterization of the silica hydride column and diamond hydride column under aqueous normal phase mode using isocratic and gradient methods. Adenosine, creatinine, L-tryptophan, guanine, guanidine and several primary amines were analyzed in the ANP isocratic mode on the silica hydride column. All samples were dissolved in 50:50 acetonitrile/water (both premixed with $0.1 \%$ formic acid) at a $0.5 \mathrm{mg} / \mathrm{mL}$ concentration. The UV detector used for the analysis was operated at $254 \mathrm{~nm}$ wavelength. The flow rate was set at $0.5 \mathrm{~mL} / \mathrm{min}$, at ambient temperature with a $5 \mu \mathrm{L}$ injection volume.

Table 8. Mobile phase Gradients A and B used for ANP analysis.

\begin{tabular}{|c|c|c|c|c|c|}
\hline \multicolumn{3}{|c|}{ Gradient A } & \multicolumn{3}{c|}{ Gradient B } \\
\hline $\begin{array}{c}\text { Time } \\
\text { (min) }\end{array}$ & $\begin{array}{c}\text { Acetonitrile } \\
\text { with 0.1\% FA }\end{array}$ & $\begin{array}{c}\text { Water } \\
\text { with } \\
\text { 0.1\% FA }\end{array}$ & Time (min) & $\begin{array}{c}\text { Acetonitrile } \\
\text { with 0.1\% FA }\end{array}$ & $\begin{array}{c}\text { Water } \\
\text { with 0.1\% } \\
\text { FA }\end{array}$ \\
\hline 0 & 85 & 15 & 0 & 75 & 25 \\
\hline 4 & 85 & 15 & 4 & 75 & 25 \\
\hline 10 & 50 & 50 & 9 & 50 & 50 \\
\hline 12 & 50 & 50 & 11 & 50 & 50 \\
\hline 14 & 85 & 15 & 14 & 75 & 25 \\
\hline 19 & 85 & 15 & 19 & 75 & 25 \\
\hline
\end{tabular}


Table 9. Mobile phase Gradients C and D used for ANP analysis.

\begin{tabular}{|c|c|c|c|c|c|}
\hline & Gradient C & & Gradient D & \\
\hline $\begin{array}{c}\text { Time } \\
\text { (min) }\end{array}$ & $\begin{array}{c}\text { Acetonitrile } \\
\text { with 0.1\% } \\
\text { FA }\end{array}$ & $\begin{array}{c}\text { Water with } \\
\mathbf{0 . 1} \% \text { FA }\end{array}$ & $\begin{array}{c}\text { Time } \\
\text { (min) }\end{array}$ & $\begin{array}{c}\text { Acetonitrile } \\
\text { with 0.1\% FA }\end{array}$ & $\begin{array}{c}\text { Water with } \\
\mathbf{0 . 1 \%} \text { FA }\end{array}$ \\
\hline 0 & 85 & 15 & 0 & 90 & 10 \\
\hline 4 & 85 & 15 & 4 & 90 & 10 \\
\hline 10 & 60 & 40 & 9 & 70 & 30 \\
\hline 12 & 60 & 40 & 11 & 70 & 30 \\
\hline 14 & 85 & 15 & 14 & 90 & 10 \\
\hline 19 & 85 & 15 & 19 & 90 & 10 \\
\hline
\end{tabular}

\subsection{ANP Behavior of Adenosine}

The analysis of adenosine using UV detection was carried out using ANP isocratic conditions. This purine nucleoside was prepared using 50:50 acetonitrile $(0.1 \%$ formic acid $) /$ water $(0.1 \%$ formic acid $)$ at a $0.5 \mathrm{mg} / \mathrm{mL}$ concentration. The flow rate was set at $0.5 \mathrm{~mL} / \mathrm{min}$ with a $5 \mu \mathrm{L}$ injection volume. A wavelength of 254 $\mathrm{nm}$ was selected for the analysis and stabilized according to the initial conditions 
of the isocratic test. Figure 18 shows the chromatograms of adenosine for $50: 50$ and 80:20 acetonitrile/water (with $0.1 \%$ formic acid) compositions.
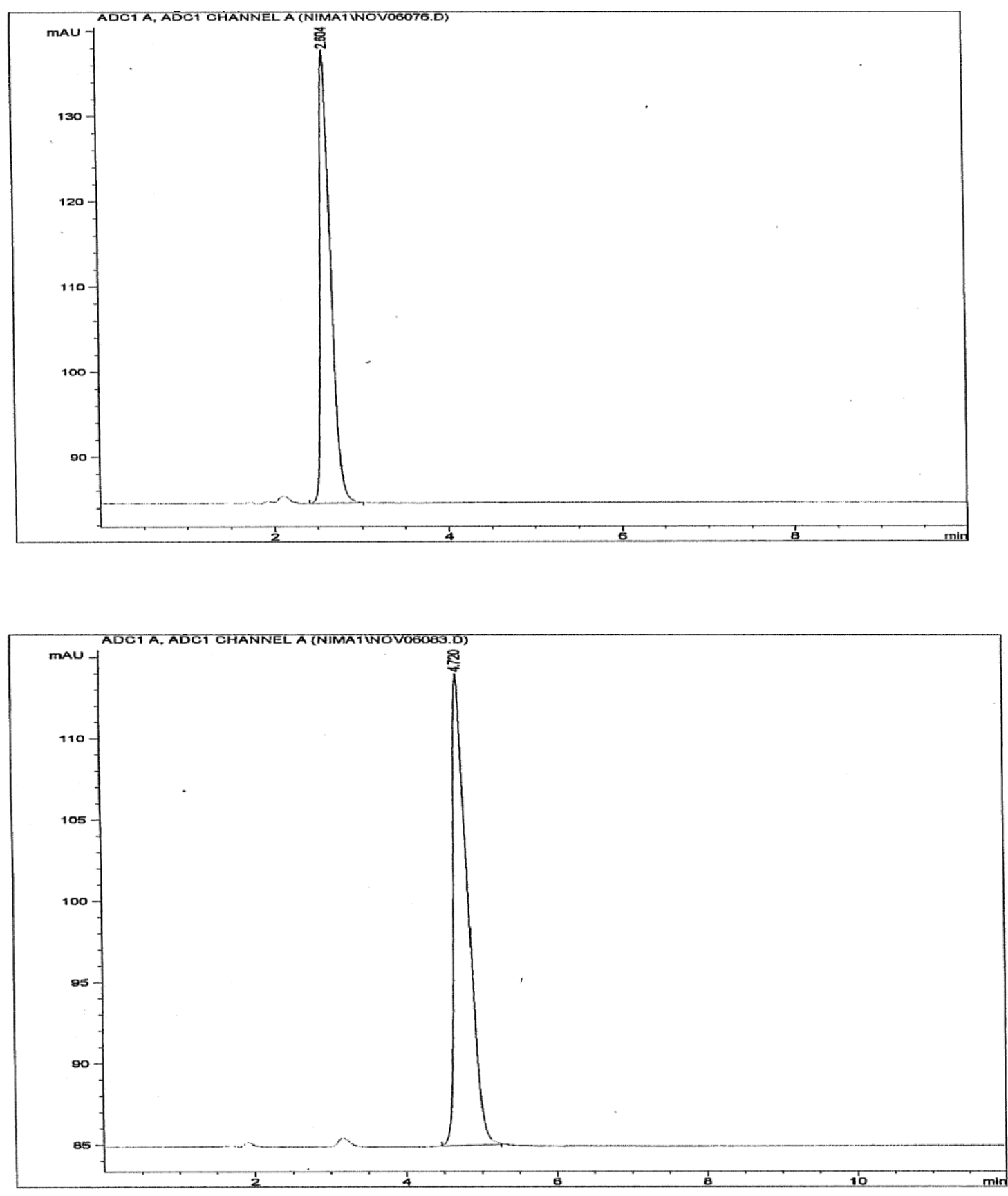

Figure 18. Chromatograms of adenosine obtained using the silica hydride column at 50:50 acetonitrile/water (Top) and 80:20 acetonitrile/water (Bottom) as mobile phase (both premixed with $0.1 \%$ formic acid). 
Table 10 lists the retention times and Figure 19 reports the corresponding characteristic retention map of adenosine observed on the silica hydride column at various \% concentrations of mobile phase compositions. As the concentration of acetonitrile increases from $50 \%$ to $80 \%$, the retention time of L-tryptophan also increases which confirms the ANP behavior of this compound.

Table 10. Retention time of adenosine on the silica hydride column using UV detector.

\begin{tabular}{|c|c|c|}
\hline \multicolumn{2}{|c|}{ Concentration (\%) } & Retention time (min) \\
\hline $\begin{array}{c}\text { Acetonitrile with 0.1\% } \\
\text { FA }\end{array}$ & DI water with 0.1\% FA & Adenosine \\
\hline 50 & 50 & 2.60 \\
\hline 60 & 40 & 2.92 \\
\hline 70 & 30 & 3.46 \\
\hline 80 & 20 & 4.72 \\
\hline 90 & 10 & 7.05 \\
\hline
\end{tabular}




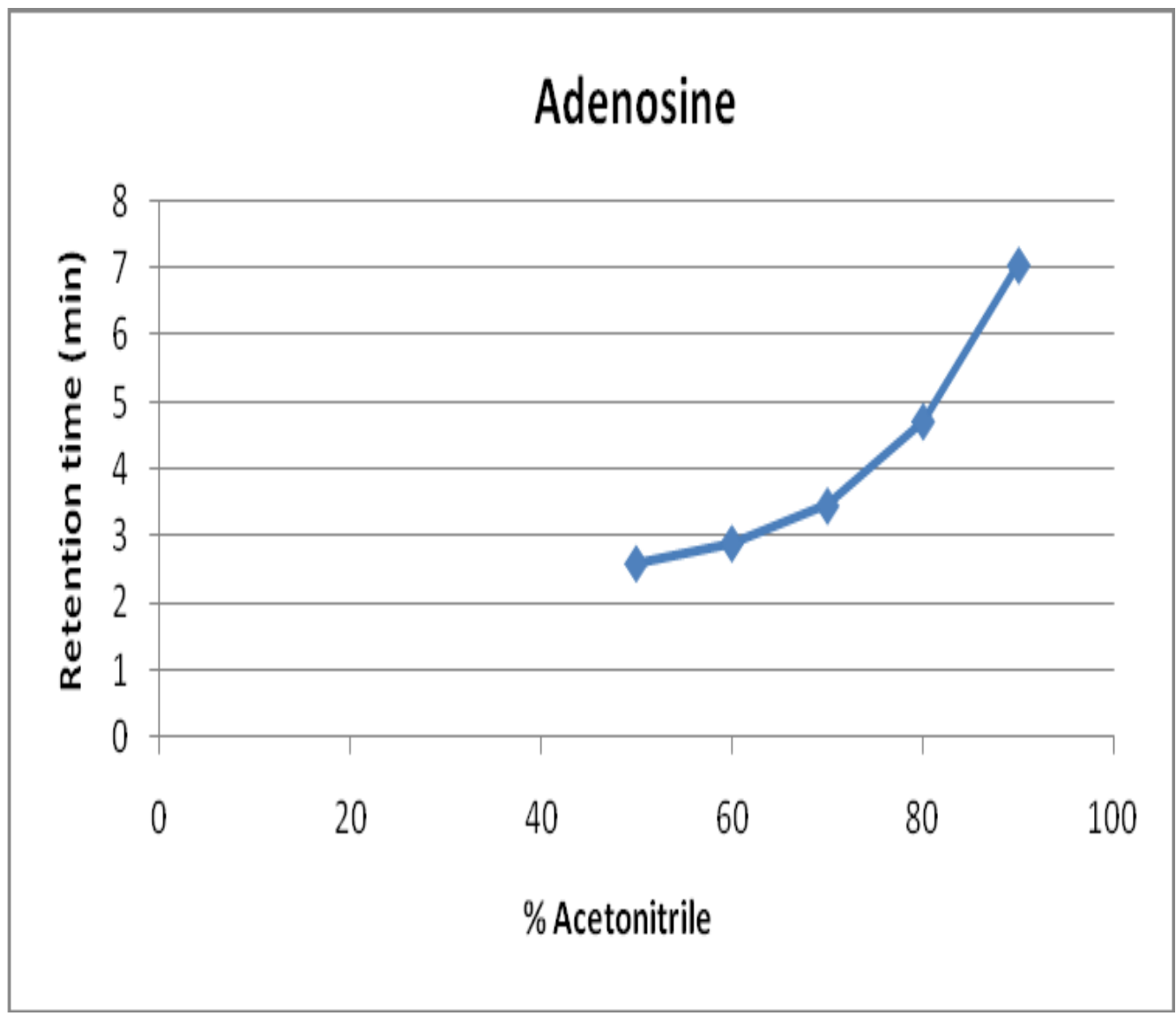

Figure 19. Retention map of adenosine on the silica hydride column using UV detection.

\subsection{ANP Behavior of Guanine}

Guanine was analyzed under ANP solvent conditions on the silica hydride column. A UV detector was used for the analysis. Guanine was difficult to dissolve so $200 \mu \mathrm{L}$ of $\mathrm{HCl}$ solution was mixed with $50: 50$ acetonitrile/water (both premixed with $0.1 \%$ formic acid) at a concentration of $0.5 \mathrm{mg} / \mathrm{mL}$. A wavelength of $254 \mathrm{~nm}$ was selected and stabilized according to the initial conditions of the isocratic test. Chromatograms of guanine for 50:50 and 80:20 acetonitrile/water (with $0.1 \%$ formic acid) compositions are shown in Figure 20. 

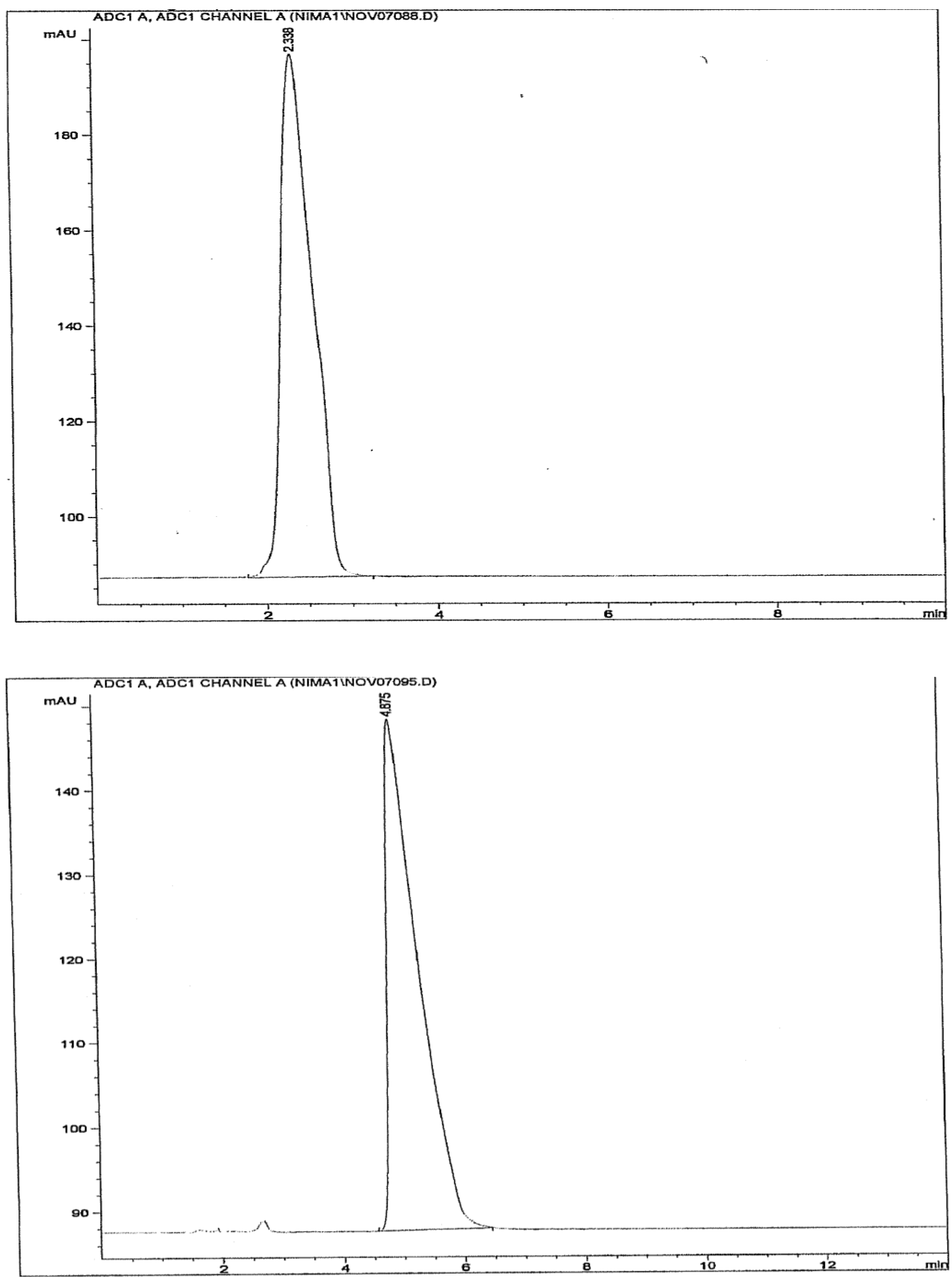

Figure 20. Chromatograms of guanine obtained using the silica hydride stationary phase at 50:50 acetonitrile/water (Top) and 80:20 acetonitrile/water (Bottom) as mobile phase (both premixed with $0.1 \%$ formic acid). 
Retention times of guanine at various $\%$ concentration of acetonitrile are listed in Table 11. It can be seen from Table 11 that guanine retained longer on the column as the concentration of the acetonitrile was increased. The retention map of guanine is shown in Figure 21. From Figure 21 it can be seen that at $90 \%$ acetonitrile concentration the compound shows significant retention which confirms that the silica hydride stationary phase is suitable for this nucleobase in the ANP mode.

Table 11. Retention time of guanine on the silica hydride column using UV detection.

\begin{tabular}{|c|c|c|}
\hline \multicolumn{2}{|c|}{ Concentration (\%) } & Retention time (min) \\
\hline $\begin{array}{c}\text { Acetonitrile with } \\
\text {..1\% FA }\end{array}$ & DI water with 0.1\% FA & Guanine \\
\hline 50 & 50 & 2.34 \\
\hline 60 & 40 & 2.64 \\
\hline 70 & 30 & 3.34 \\
\hline 80 & 20 & 4.88 \\
\hline 90 & 10 & 10.95 \\
\hline
\end{tabular}




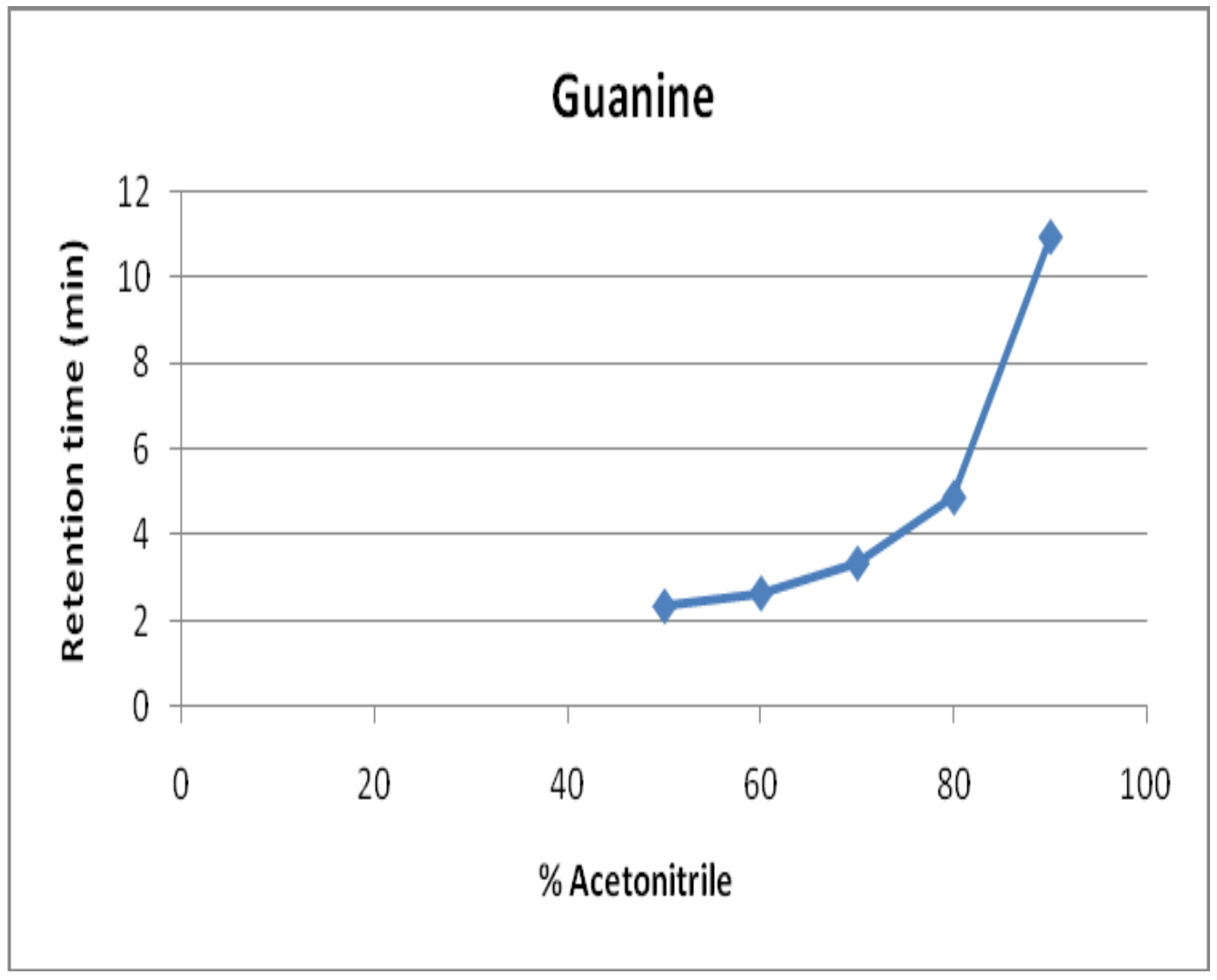

Figure 21. Retention map of guanine on the silica hydride column using UV detection.

\subsection{ANP Behavior of Creatinine}

Creatinine was analyzed using UV detection under ANP isocratic conditions. The compound was dissolved using 50:50 acetonitrile/water (both premixed with $0.1 \%$ formic acid) at a concentration of $0.5 \mathrm{mg} / \mathrm{mL}$. The UV detector was set at $254 \mathrm{~nm}$ and stabilized according to the initial isocratic conditions. 
A chromatogram of creatinine for a $50: 50$ mobile phase composition is shown in Figure 22. Retention times of creatinine at various \% concentration of acetonitrile are listed in Table 12. It was observed that as the concentration of organic solvent was increased from $50 \%$ to $80 \%$, the retention time of creatinine gradually increased. The characteristic ANP retention map of creatinine is shown Figure 23. This data obtained indicates the typical ANP behavior of creatinine on the silica hydride column.

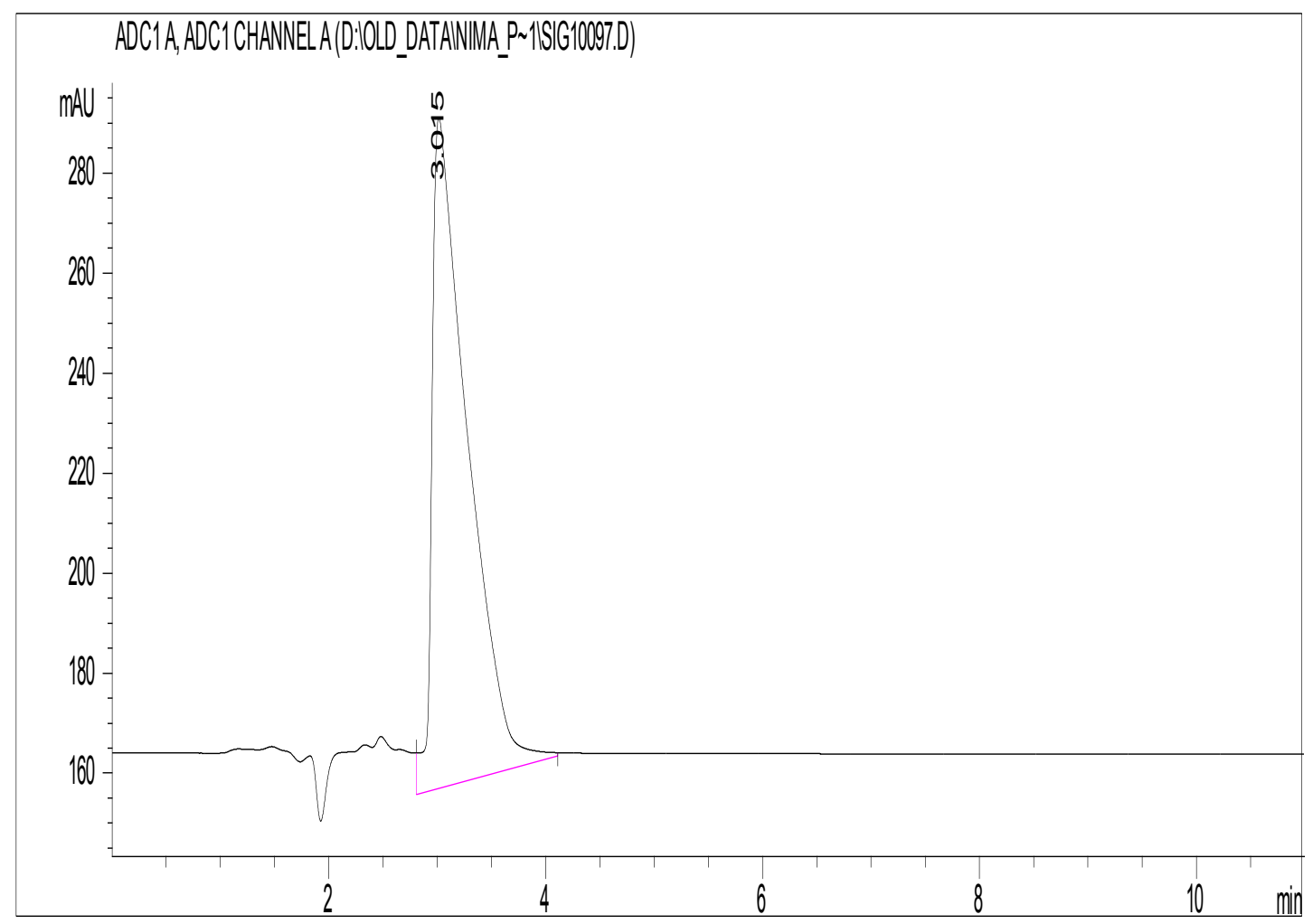

Figure 22. Chromatogram of creatinine on the silica hydride stationary phase. Mobile phase: $50: 50$ acetonitrile $+0.1 \%$ formic acid / water $+0.1 \%$ formic acid. 
Table 12. Retention time of creatinine on the silica hydride column using UV detector.

\begin{tabular}{|c|c|c|}
\hline \multicolumn{2}{|c|}{ Concentration(\%) } & Retention time (min) \\
\hline $\begin{array}{l}\text { Acetonitrile with 0.1\% } \\
\text { FA }\end{array}$ & DI water with 0.1\% FA & Creatinine \\
\hline 50 & 50 & 3.01 \\
\hline 60 & 40 & 3.63 \\
\hline 70 & 30 & 4.99 \\
\hline 80 & 20 & 6.80 \\
\hline
\end{tabular}

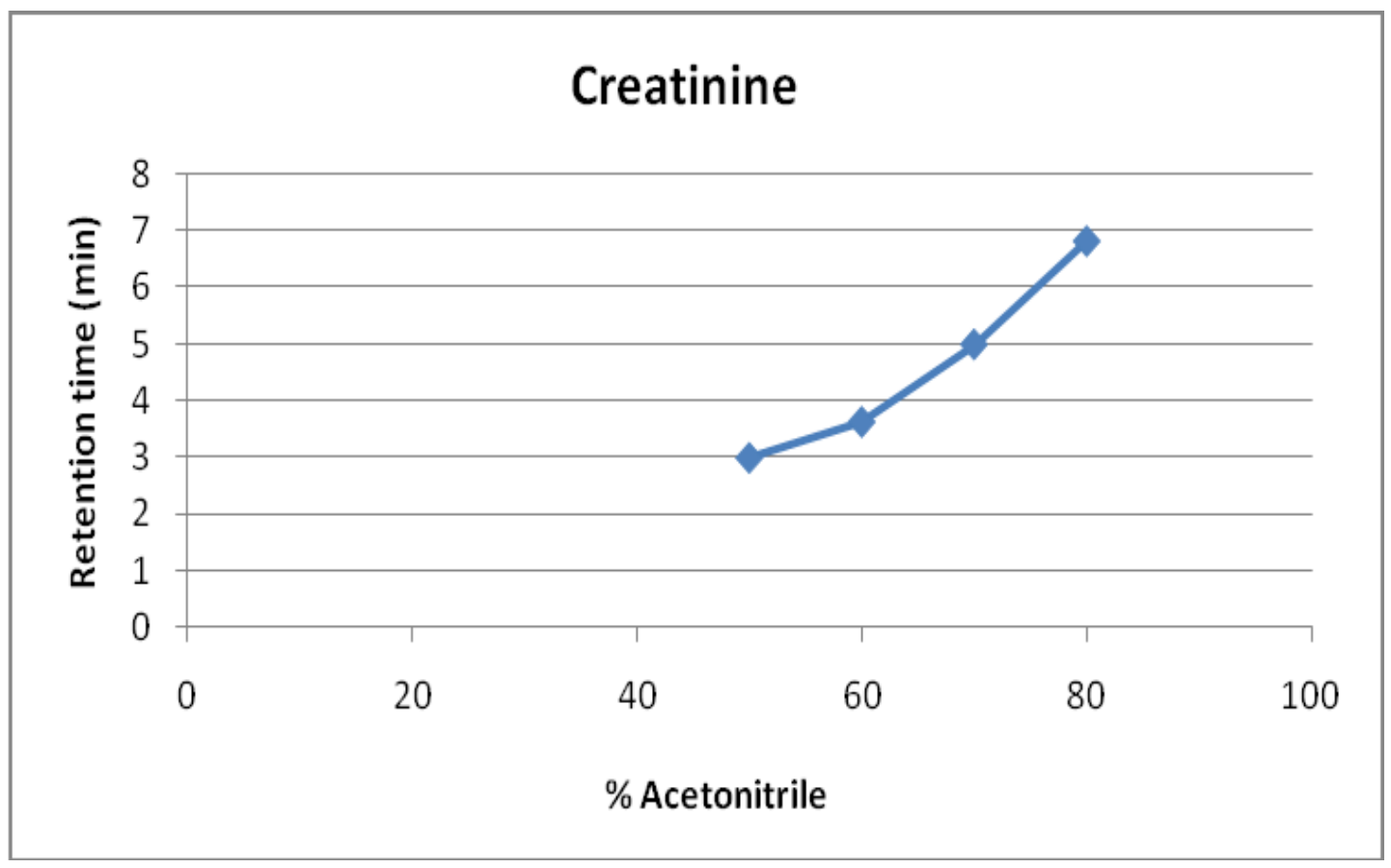

Figure 23. Retention map of creatinine on the silica hydride column using UV detection. 


\subsection{ANP Behavior of Guanidine}

Guanidine was analyzed using UV detection under different ANP isocratic conditions as shown in Figure 24. The compound was dissolved using 50:50 acetonitrile/water (both premixed with $0.1 \%$ formic acid) at a concentration of $0.5 \mathrm{mg} / \mathrm{mL}$. The UV detector was set at $254 \mathrm{~nm}$ and stabilized according to the initial isocratic conditions.

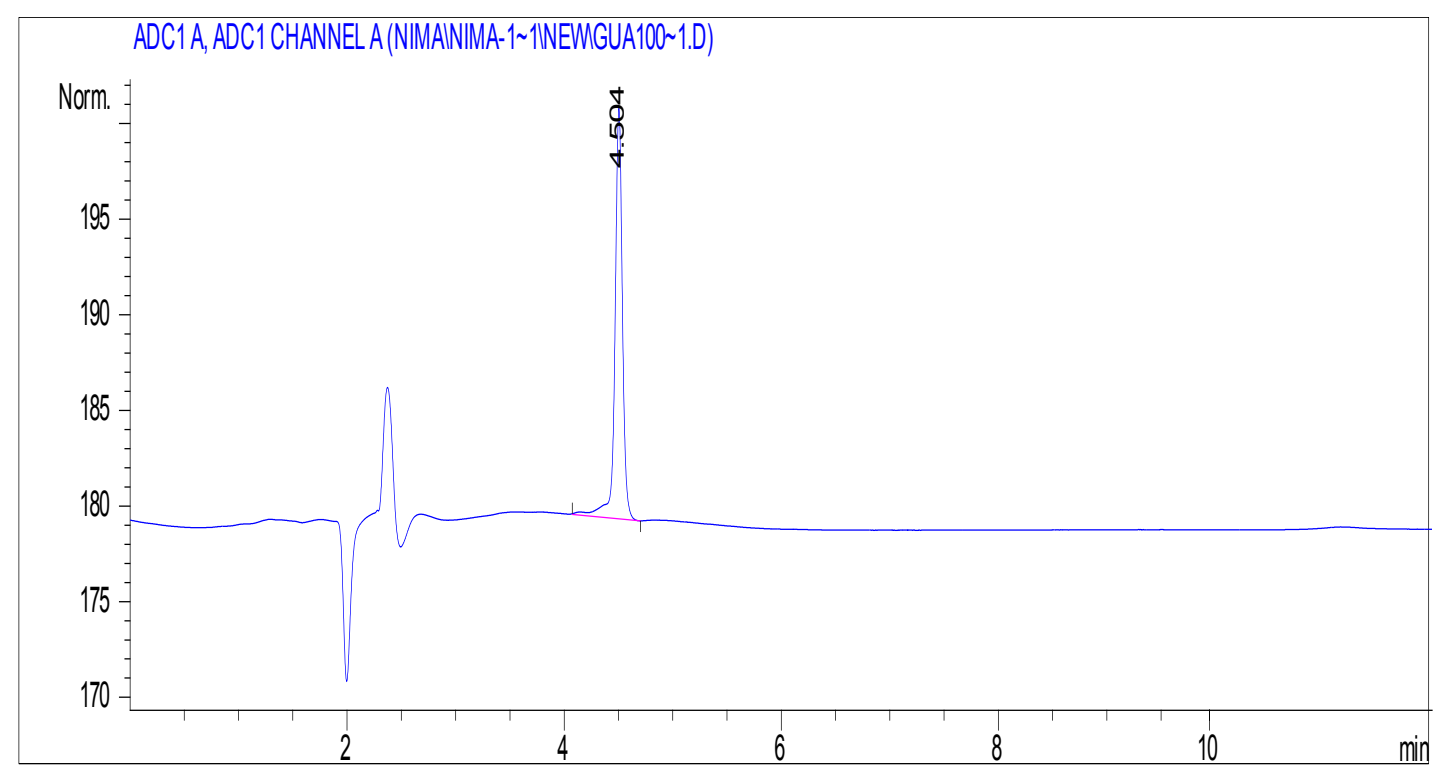

Figure 24. Chromatogram of guanidine on the silica hydride stationary phase. Mobile phase: $50: 50$ acetonitrile $+0.1 \%$ formic acid / water $+0.1 \%$ formic acid.

Table 13 shows ANP retention data of guanidine and Figure 25 is the retention map of guanidine which confirms the characteristic aqueous normal phase behavior as it is retained longer on the column with increased concentration of acetonitrile. Guanidine shows the longest retention on the 
column for $80: 20$ acetonitrile/water (with $0.1 \%$ formic acid) composition. This data confirms the ANP behavior of guanidine on the silica hydride column.

Table 13. Retention time of guanidine on the silica hydride column using UV detector.

\begin{tabular}{|c|c|c|}
\hline \multicolumn{2}{|c|}{ Concentration (\%) } & Retention time (min) \\
\hline Acetonitrile with 0.1\% FA & DI water with 0.1\% FA & Guanidine \\
\hline 50 & 50 & 4.50 \\
\hline 60 & 40 & 6.03 \\
\hline 70 & 30 & 8.90 \\
\hline 80 & 20 & 16.27 \\
\hline
\end{tabular}

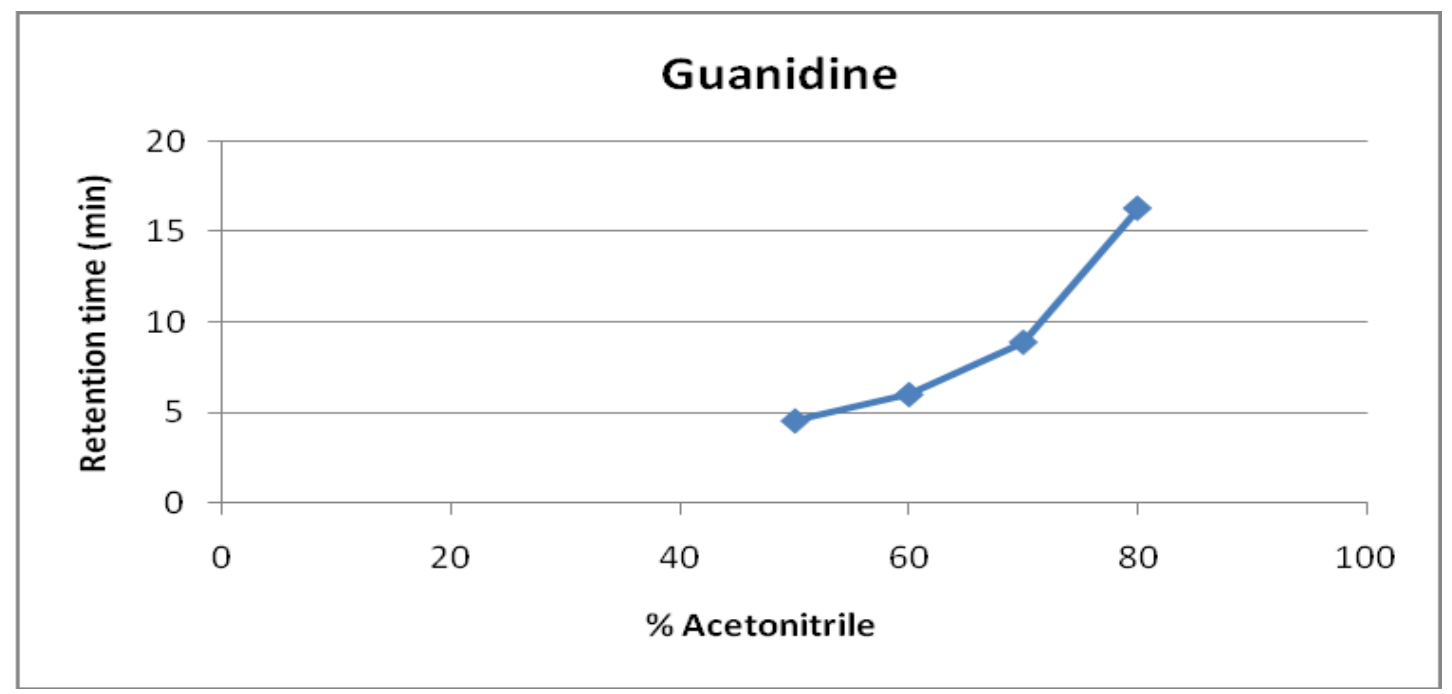

Figure 25. Retention map of guanidine on the silica hydride column using UV detection. 


\subsection{ANP Behavior of L-Tryptophan}

The amino acid, L-tryptophan was analyzed using UV detection under different ANP isocratic conditions, as shown in Table 14. A chromatogram of Ltryptophan for $80: 20$ acetonitrile/water (with $0.1 \%$ formic acid) composition is shown in Figure 26. The concentration of the sample was $0.5 \mathrm{mg} / \mathrm{mL}$ in $50: 50$ acetonitrile/water (both premixed with $0.1 \%$ formic acid). The flow rate was set at $0.5 \mathrm{~mL} / \mathrm{min}$ at ambient temperature with a $5 \mu \mathrm{L}$ injection volume. The UV detector was set at $254 \mathrm{~nm}$ and stabilized according to the initial isocratic conditions. Based on Table 14, it can be concluded that L-tryptophan showed very good retention on the column. It can be seen from Figure 27 that the retention of Ltryptophan increased with an increase in percentage of acetonitrile in the mobile phase. These data confirmed the ANP behavior of L-tryptophan on the silica hydride column.

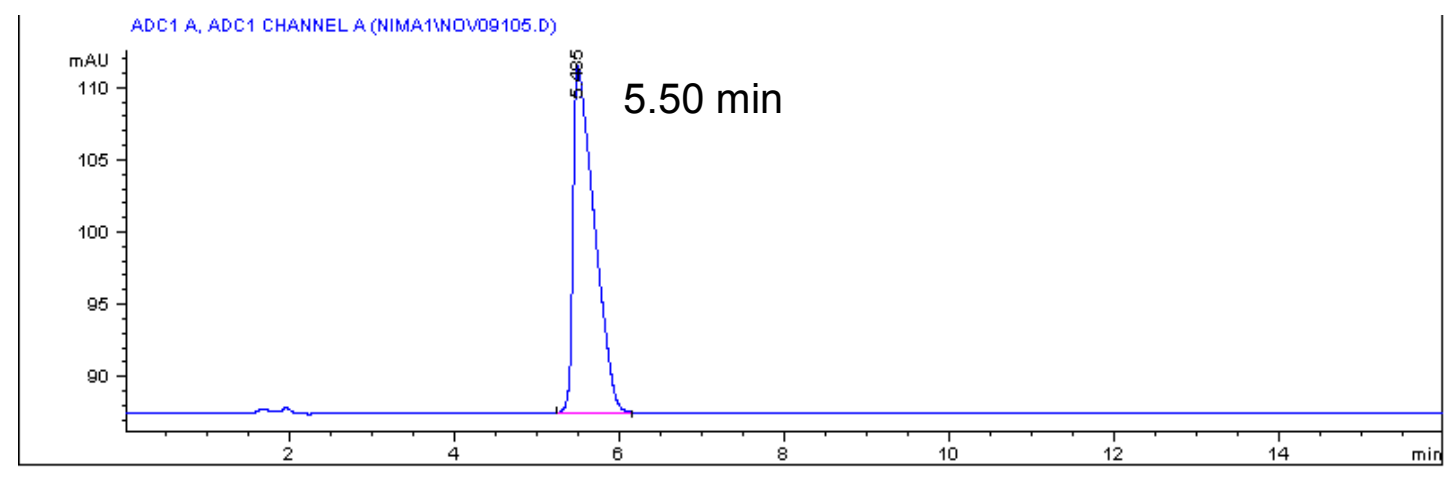

Figure 26. Chromatogram of L-tryptophan on the silica hydride stationary phase. Mobile phase: $80: 20$ acetonitrile $+0.1 \%$ formic acid / water $+0.1 \%$ formic acid. 
Table 14. Retention time of L-tryptophan on the silica hydride column using UV detector.

\begin{tabular}{|c|c|c|}
\hline \multicolumn{2}{|c|}{ Concentration (\%) } & Retention time (min) \\
\hline Acetonitrile with 0.1\% FA & Dl water with 0.1\% FA & L-Tryptophan \\
\hline 50 & 50 & 2.45 \\
\hline 60 & 40 & 2.75 \\
\hline 70 & 30 & 3.46 \\
\hline 80 & 20 & 5.50 \\
\hline 90 & 10 & 9.17 \\
\hline
\end{tabular}

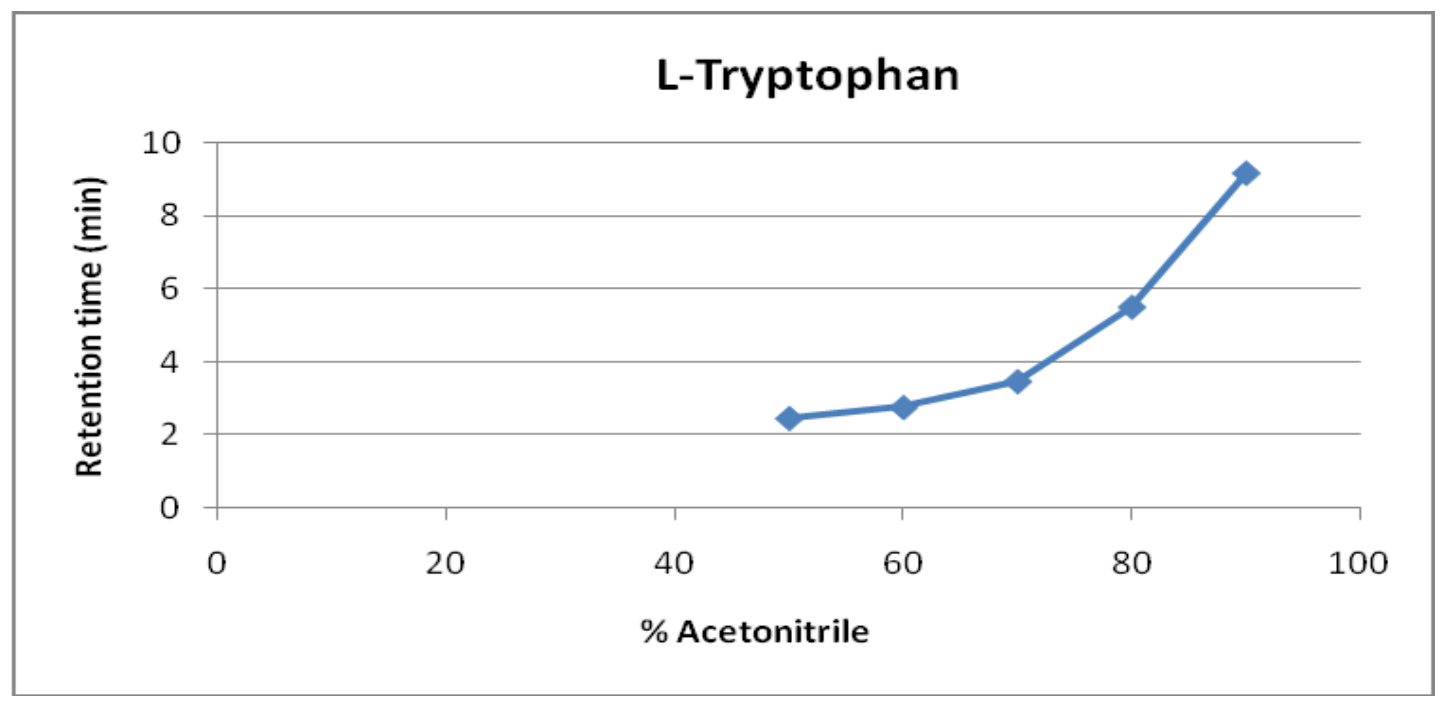

Figure 27. Retention map of L-tryptophan on the silica hydride column using UV detection.

\subsection{ANP Isocratic Behavior of Primary Amines}

The primary amines 2,6-diaminopurine, 2-phenethylamine, benzylamine, and 1,2,4,5-benzenetetracarboxamide were analyzed under aqueous normal 
phase isocratic conditions on the silica hydride column as shown in Figures 28 - 31 respectively. 1,2,4,5-Benzenetetramine tetrahydrochloride and 4-chloro1,2-phenylenediamine were also analyzed under aqueous normal phase conditions on the silica hydride column, as summarized in Table 15. All primary amines were prepared in 50:50 acetonitrile/water (both premixed with $0.1 \%$ formic acid) at a concentration of $0.5 \mathrm{mg} / \mathrm{mL}$. The detector wavelength was 254 $\mathrm{nm}$, and the system was stabilized according to the initial isocratic conditions. The flow rate used was $0.5 \mathrm{~mL} / \mathrm{min}$ with a $5 \mu \mathrm{L}$ injection volume at ambient temperature. Tables 15 and 16 show a summary of isocratic retention of primary amines on the silica hydride column. It can be seen that the retention times of all polar primary amines increased with increasing concentration of acetonirile, which is the typical ANP behavior of these compunds on a silica hydride column.

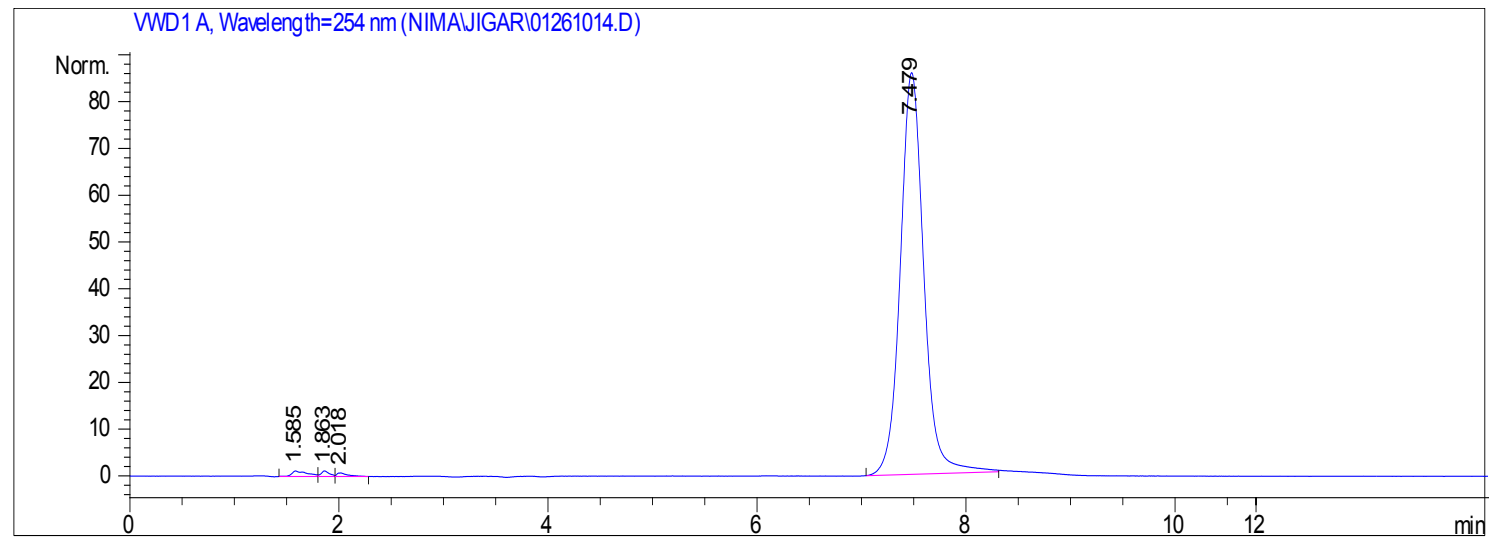

Figure 28. Chromatogram of 2,6-diaminopurine on the silica hydride stationary phase. Mobile phase: $80: 20$ acetonitrile $+0.1 \%$ formic acid $/$ water $+0.1 \%$ formic acid. 


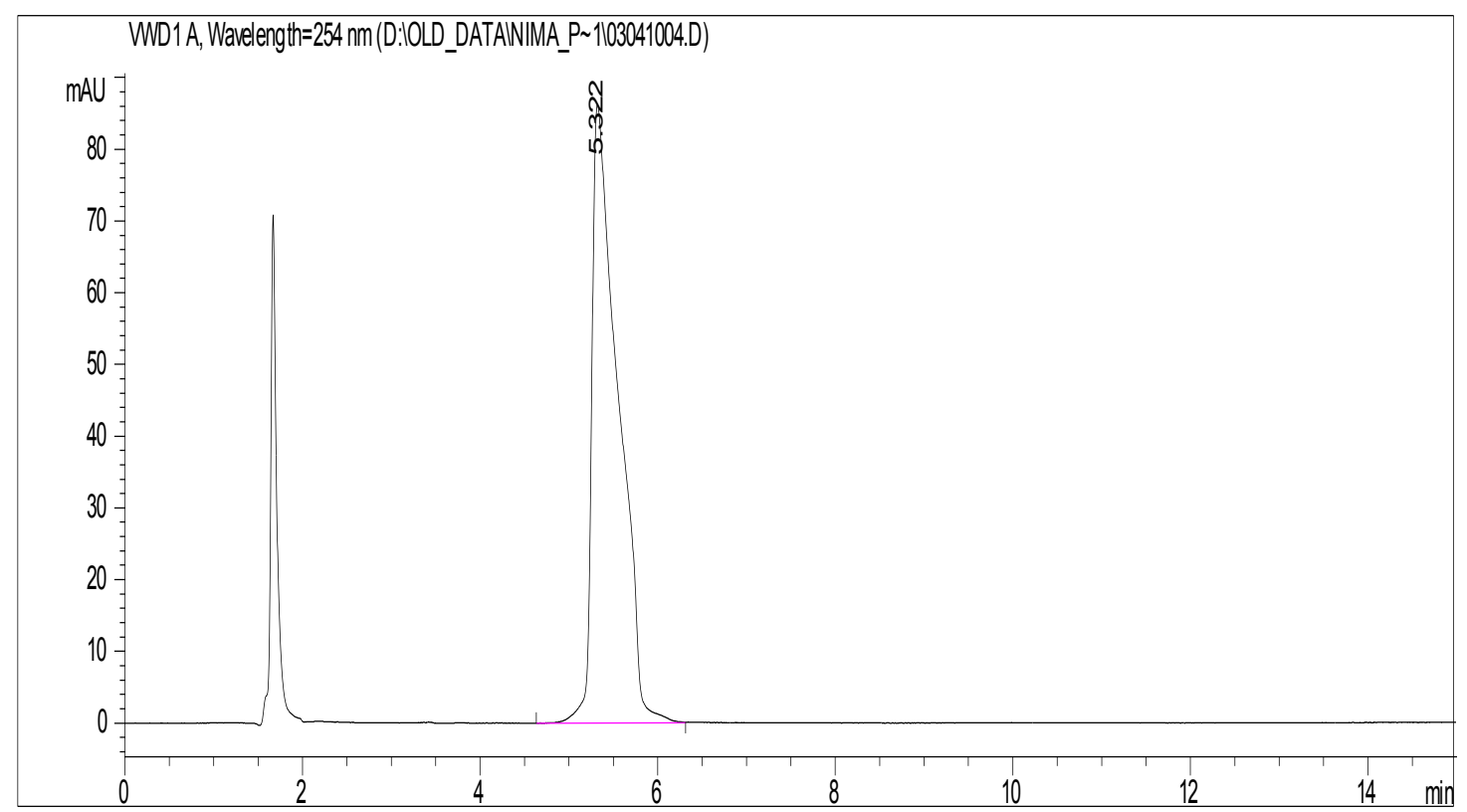

Figure 29. Chromatogram of benzylamine on the silica hydride stationary phase. Mobile phase: $80: 20$ acetonitrile $+0.1 \%$ formic acid / water $+0.1 \%$ formic acid.

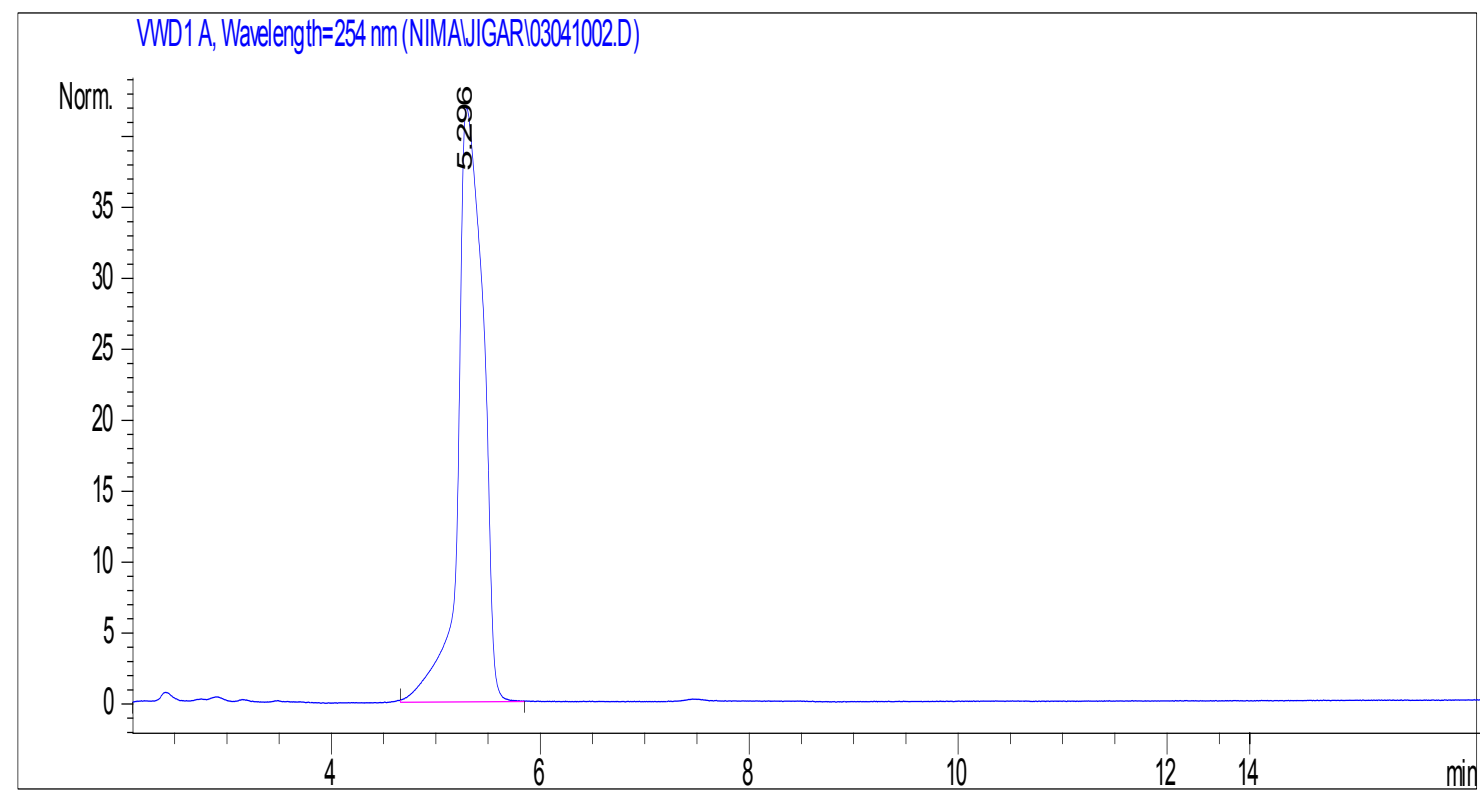

Figure 30. Chromatogram of 2-phenethylamine on the silica hydride stationary phase. Mobile phase: $80: 20$ acetonitrile $+0.1 \%$ formic acid $/$ water $+0.1 \%$ formic acid. 


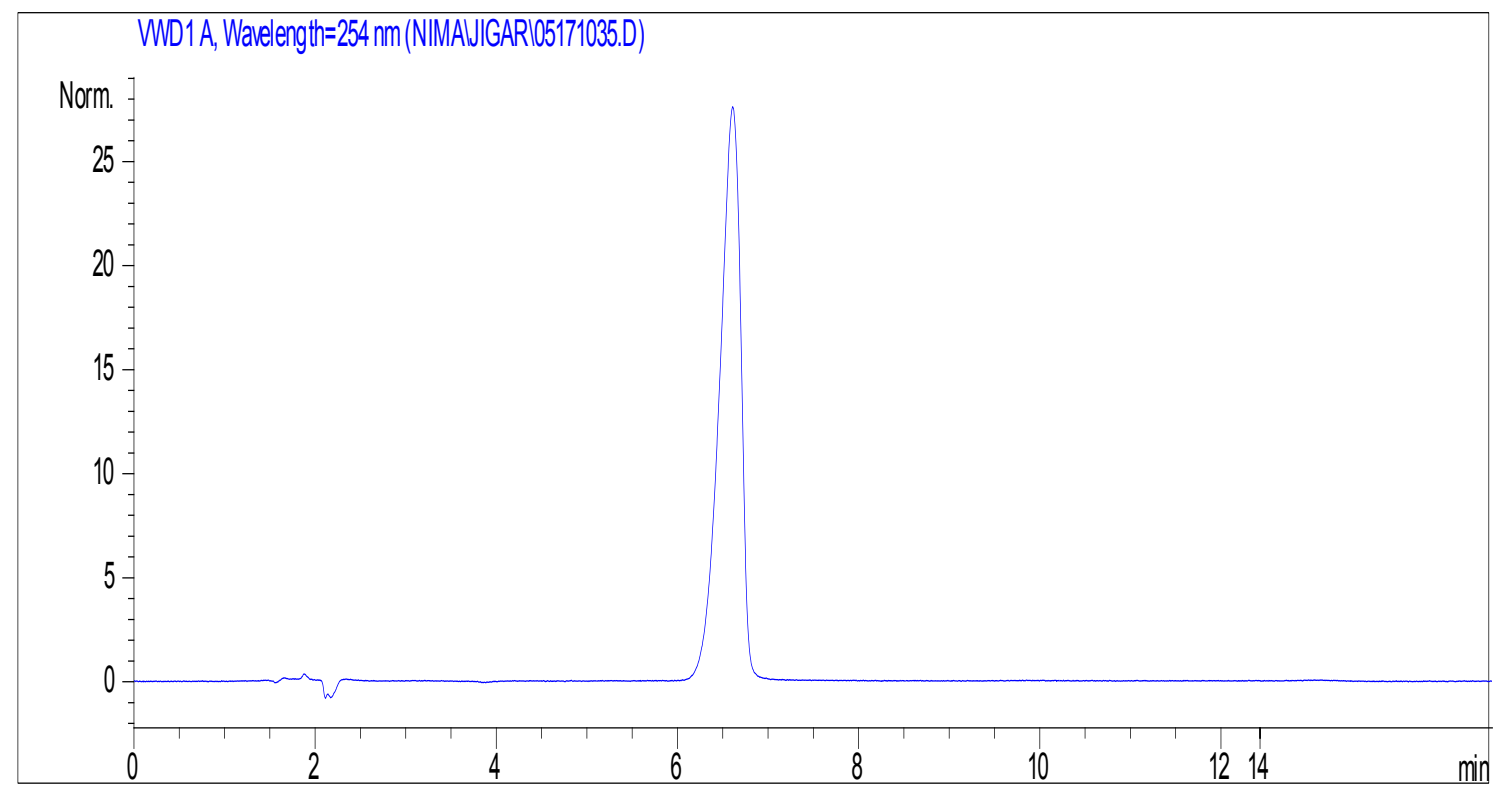

Figure 31. Chromatogram of 1,2,4,5-benzenetetracarboxamide on the silica hydride stationary phase. Mobile phase: $80: 20$ acetonitrile $+0.1 \%$ formic acid / water $+0.1 \%$ formic acid.

Table 15. Summary of isocratic elution data for primary amines.

\begin{tabular}{|c|c|c|c|c|}
\hline \multicolumn{2}{|c|}{ Concentration (\%) } & \multicolumn{3}{c|}{ Retention time(min) } \\
\hline $\begin{array}{c}\text { Acetonitrile } \\
\text { (0.1\% FA) }\end{array}$ & $\begin{array}{c}\text { Water } \\
\text { (0.1\% FA) }\end{array}$ & $\begin{array}{c}\mathbf{2 , 6 -} \\
\text { Diaminopurine }\end{array}$ & $\begin{array}{c}\text { 4-Chloro-1,2- } \\
\text { phenethylenediamine }\end{array}$ & $\begin{array}{c}\mathbf{1 , 2 , 4 , 5 -} \\
\text { Benzenetetramine- } \\
\text { tetrahydrochloride }\end{array}$ \\
\hline 50 & 50 & 3.23 & 2.64 & 3.07 \\
\hline 60 & 40 & 3.91 & 2.82 & 3.63 \\
\hline 70 & 30 & 5.06 & 3.24 & 4.51 \\
\hline 80 & 20 & 7.47 & 4.83 & 6.48 \\
\hline 90 & 10 & - & 12.37 & - \\
\hline
\end{tabular}


Table 16. Summary of isocratic elution data for Primary amines.

\begin{tabular}{|c|c|c|c|c|}
\hline \multicolumn{2}{|c|}{ Concentration (\%) } & \multicolumn{3}{|c|}{ Retention time(min.) } \\
\hline $\begin{array}{c}\text { Acetonitrile } \\
\mathbf{( 0 . 1 \%} \text { FA) }\end{array}$ & $\begin{array}{c}\text { Water } \\
(\mathbf{0 . 1 \%} \text { FA) }\end{array}$ & Benzylamine & 2-Phenethylamine & $\begin{array}{c}\mathbf{1 , 2 , 4 , 5 - B e n z e n e - ~} \\
\text { tetracarboxamide }\end{array}$ \\
\hline 50 & 50 & 2.58 & 2.56 & 1.79 \\
\hline 60 & 40 & 2.9 & 2.88 & 1.86 \\
\hline 70 & 30 & 3.59 & 3.51 & 1.96 \\
\hline 80 & 20 & 5.32 & 5.29 & 2.21 \\
\hline 90 & 10 & 16.43 & 17.09 & 3.27 \\
\hline
\end{tabular}

Besides these compounds, one more primary amine, 3,4-

diaminobenzophenone, was analyzed using the same ANP conditions and mobile phase. As shown in Table 17, 3,4-diaminobenzophenone was not retained on the silica hydride column eluting at the void volume for increasing acetonitrile concentrations. The insignificant ANP retention of this primary amine is likely due to the stated experimental condition or it is possible that the presence of two aromatic rings makes the compound too hydrophobic for ANP retention. Therefore, a conclusion was drawn that this primary amine cannot be retained on the silica hydride column using the stated experimental conditions. Further work needs to be done in order to determine if this compound can be retained on the silica hydride column. More work has been done under ANP gradient conditions which are discussed in a subsequent section. 
Table 17. Summary of isocratic elution data for 3,4-diaminobenzophenone.

\begin{tabular}{|c|c|c|}
\hline \multicolumn{2}{|c|}{ Concentration (\%) } & Retention time (min) \\
\hline $\begin{array}{c}\text { Acetonitrile with } \mathbf{0 . 1 \%} \\
\text { FA }\end{array}$ & DI water with 0.1\% FA & $\begin{array}{c}\text { 3,4- } \\
\text { Diaminobenzophenone }\end{array}$ \\
\hline 50 & 50 & 2.16 \\
\hline 60 & 40 & 2.11 \\
\hline 70 & 30 & 2.02 \\
\hline 80 & 20 & 2.13 \\
\hline 90 & 10 & 2.18 \\
\hline
\end{tabular}

\subsection{ANP Behavior of Si-H Column in Gradient Mode Using UV Detector}

Primary amines were analyzed in the isocratic and gradient modes.

Tables 8 and 9 lists the gradient used for the analysis. The flow rate was set at

$0.5 \mathrm{~mL} / \mathrm{min}$ with a $5 \mu \mathrm{L}$ injection volume at ambient temperature. A wavelength of $254 \mathrm{~nm}$ was selected and the column stabilized according to the initial conditions of the gradient mode. All primary amine samples were dissolved in 50:50 acetonitrile/water (both premixed with $0.1 \%$ formic acid) at a concentration of $0.5 \mathrm{mg} / \mathrm{mL}$. A water blank was run to remove any contamination from the previous measurements. The retention time varied from gradient to gradient depending on the increment of the percent organic component in the mobile 
phase composition. The main goal of this part of the research was to study the silica hydride stationary phase for aqueous normal phase behavior.

2,6-Diaminopurine was analyzed using Gradient B as shown in Table 8. The chromatogram in Figure 32 shows the gradient retention of 2,6diaminopurine. It can be seen from the figure that 2,6-diaminopurine showed retention at 6.34 minutes for Gradient B. The mobile phase used for this analysis was acetonitrile ( $0.1 \%$ formic acid) and water ( $0.1 \%$ formic acid). The concentration used was $0.5 \mathrm{mg} / \mathrm{mL}$ in acetonitrile/water (both premixed with $0.1 \%$ formic acid). The flow rate was set at $0.5 \mathrm{~mL} / \mathrm{min}$ with a $5 \mu \mathrm{L}$ injection volume at ambient temperature. The analysis was carried out at $254 \mathrm{~nm}$ and the column was stabilized with the starting conditions of gradient system. Figure 32 demonstrated the retention capabilities of the silica hydride column for polar compounds using gradient aqueous normal phase conditions.

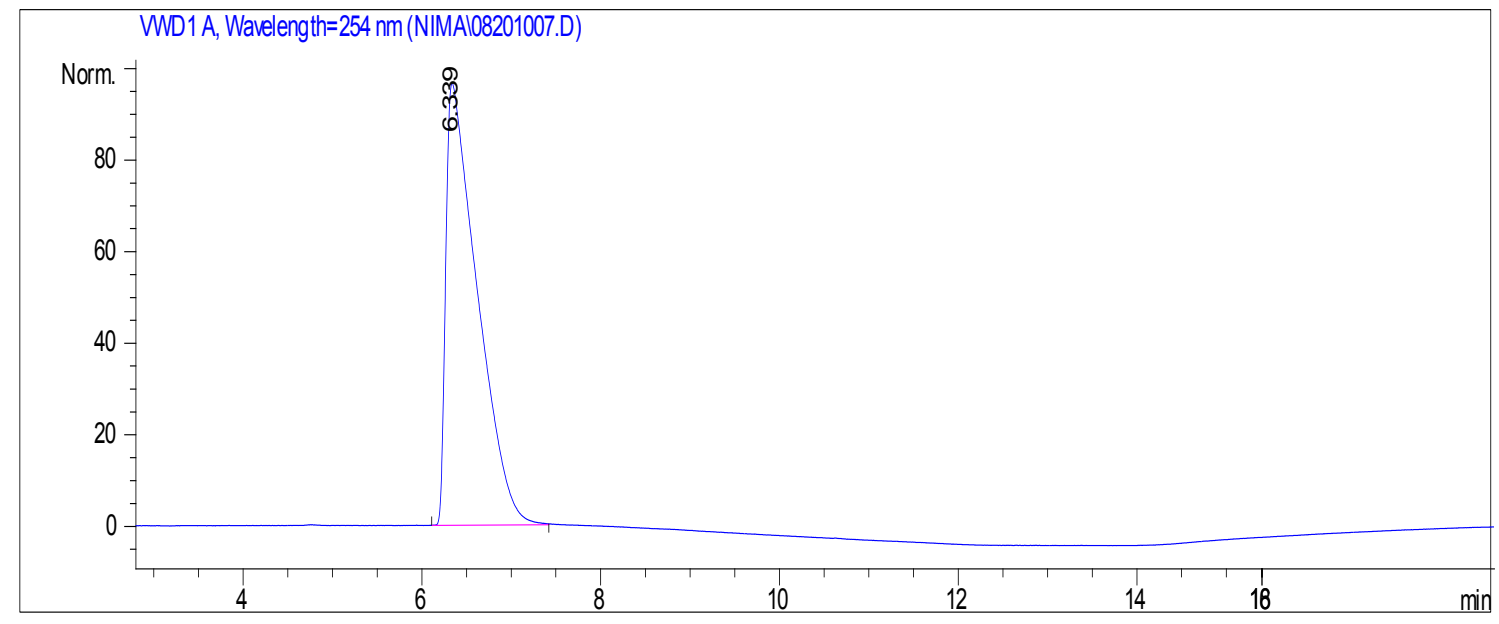

Figure 32. Chromatogram of 2,6-diaminopurine on the silica hydride column. Mobile phase Gradient B. 
As shown in Figure 33, 1,2,4,5-benzenetetramine tetrahydrochloride eluted at 3.37 minutes using Gradient A. The gradient program used is shown in Table 8. The mobile phase used was acetonitrile/water (both premixed with $0.1 \%$ formic acid). A wavelength of $254 \mathrm{~nm}$ was used and the column was stabilized according to the initial conditions of the gradient mode. A $0.5 \mu \mathrm{L} / \mathrm{min}$ flow rate was set with $5 \mu \mathrm{L}$ injection volume at ambient temperature.

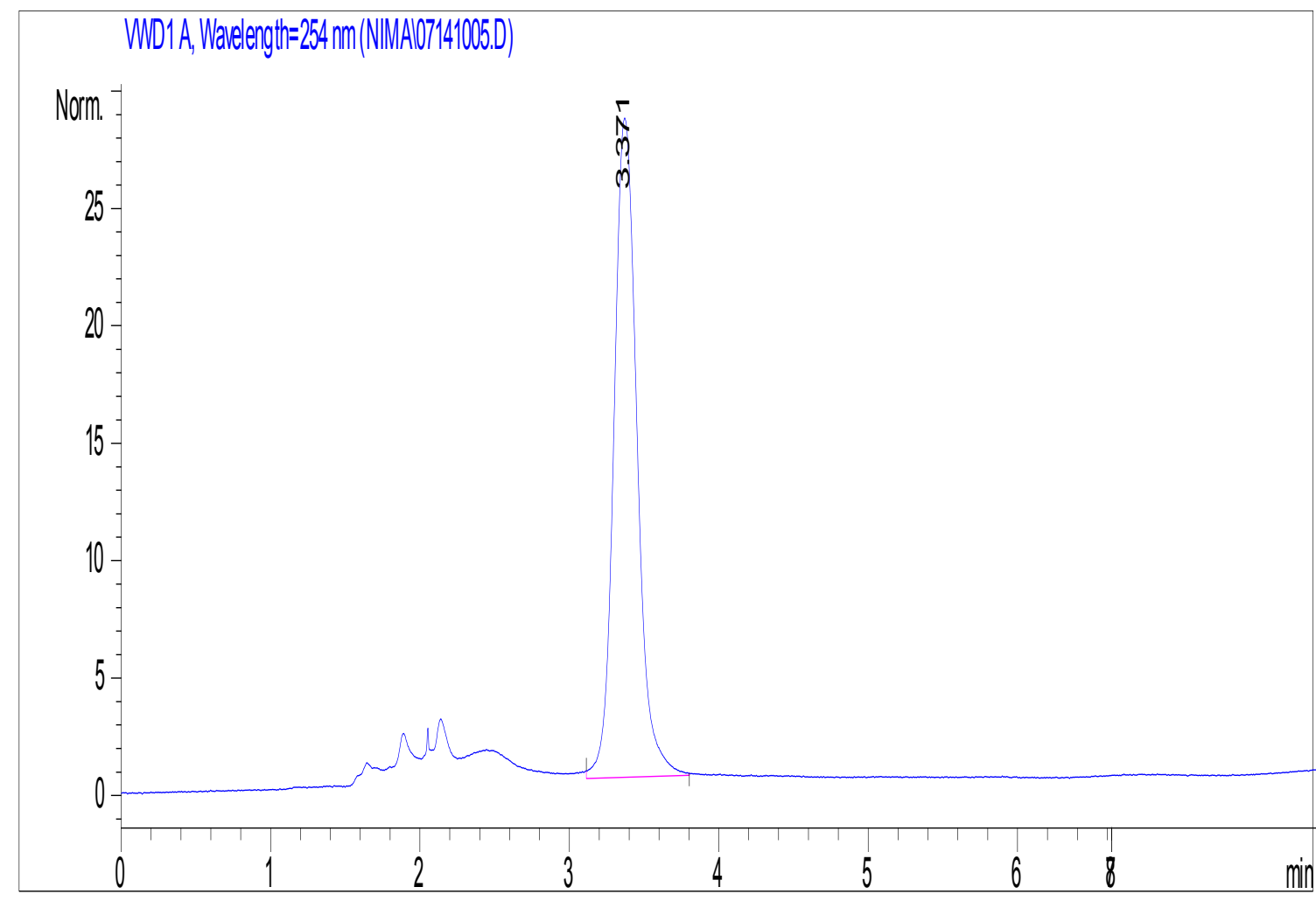

Figure 33. Chromatogram of 1,2,4,5-benzenetetramine tetrahydrochloride on the silica hydride column. Mobile phase Gradient A.

4-Chloro-1,2-phenylenediamine was tested using Gradient D and had a retention time of 3.41 minutes as shown in Figure 34. The flow rate used was 
$0.5 \mu \mathrm{L} / \mathrm{min}$ and the injection volume was $5 \mu \mathrm{L}$ at ambient temperature. Detection was done at $254 \mathrm{~nm}$ wavelength and the column was stabilized according to the starting conditions of gradient. The mobile phase used was acetonitrile/water (both premixed with $0.1 \%$ formic acid). The chromatogram in Figure 34 shows one small peak along with solvent front and two small peaks after the main peak which might be due to a sample impurity introduced during the sample preparation because water blank didn't show any extra peaks. Table 18 shows retention times for all the primary amines obtained using different gradient methods.

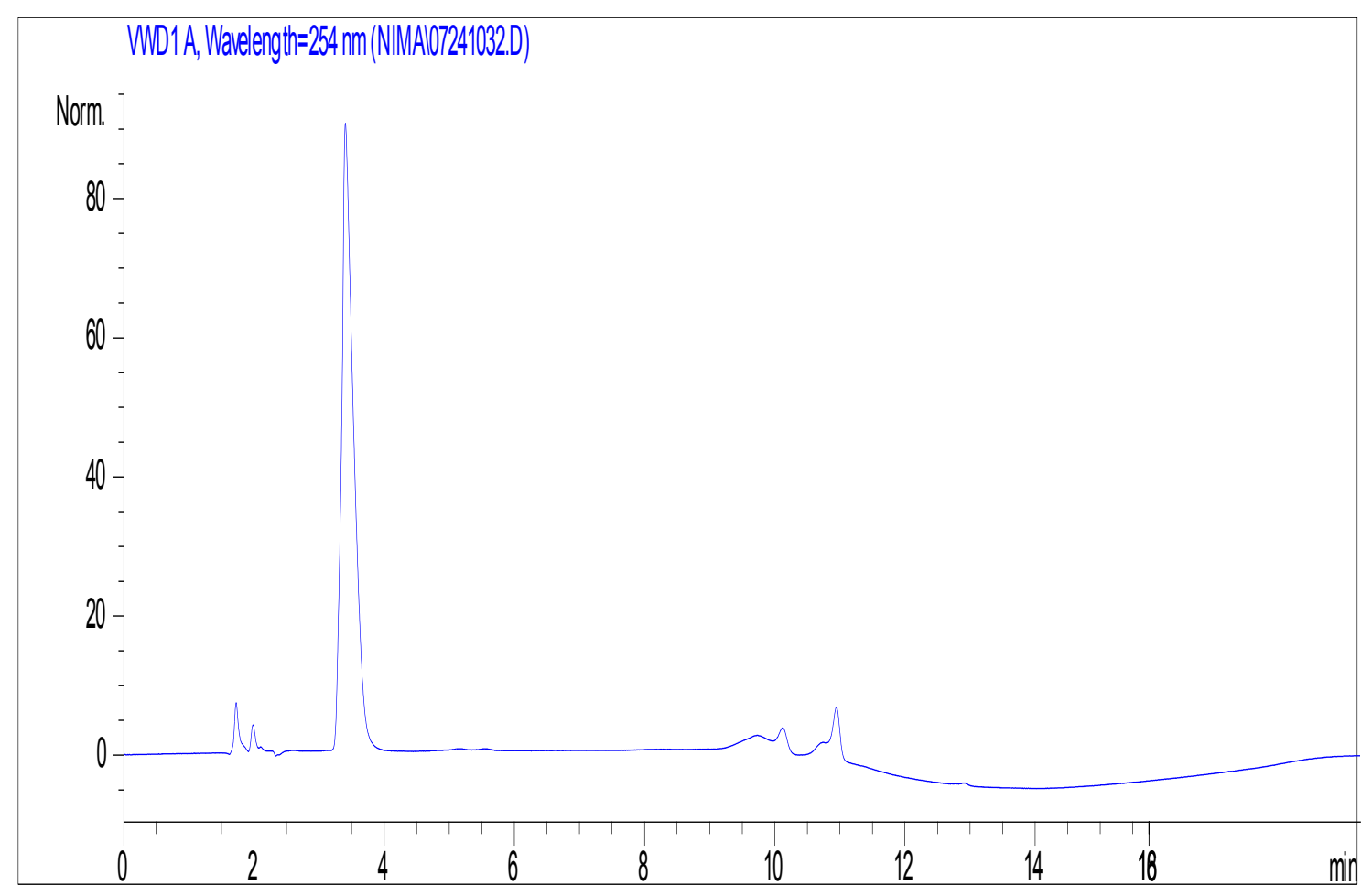

Figure 34. Chromatogram of 4-Chloro-1,2-phenylenediamine on the silica hydride column. Mobile phase Gradient D. 
Table 18. Retention times of primary amines on the silica hydride column.

\begin{tabular}{|c|c|c|c|c|}
\hline \multirow{2}{*}{ Primary amine } & \multicolumn{4}{|c|}{ Retention time } \\
\cline { 2 - 5 } & $\begin{array}{c}\text { Gradient } \\
\text { A }\end{array}$ & $\begin{array}{c}\text { Gradient } \\
\text { B }\end{array}$ & $\begin{array}{c}\text { Gradient } \\
\text { C }\end{array}$ & $\begin{array}{c}\text { Gradient } \\
\text { D }\end{array}$ \\
\hline 2,6-Diaminopurine & 10.93 & 6.34 & 11.10 & 12.45 \\
\hline 2-Phenethylamine & 9.2 & 4.02 & 8.08 & 11.16 \\
\hline Benzylamine & 8.15 & 3.97 & 8.48 & 11.17 \\
\hline $\begin{array}{c}\text { 4-Chloro-1,2-phenylenediamine } \\
\text { 1,2,4,5-Benzenetetramine- } \\
\text { tetrahydrochloride }\end{array}$ & 3.39 & 2.74 & 3.28 & 3.41 \\
\hline & 3.37 & 2.58 & 3.34 & 4.39 \\
\hline
\end{tabular}

\subsection{ANP Separation of Two Compounds Using a Gradient Method}

All the compounds used for the gradient elution were already analyzed using isocratic conditions so their behavior and retention were known on the silica hydride column. Different gradient conditions were created to separate different mixtures of primary amines. The retention time varied from gradient to gradient depending on the increment of the percent organic component in the mobile phase composition. The retention time for each of the compounds was confirmed by running the single analyte using the same mobile phase, gradient, instrument and all other parameters. The main goal of this work was to study the behavior of the silica hydride stationary phase for aqueous normal phase behavior. 
As shown in Figure 35, the two compound mixture of 1,2,4,5-

benzenetetramine tetrahydrochloride and 2,6-diaminopurine was separated using Gradient A listed in Table 8. Both compounds were dissolved in 50:50 acetonitrile $(0.1 \%$ formic acid $) /$ water $(0.1 \%$ formic acid $)$ at a concentration of $0.5 \mathrm{mg} / \mathrm{mL}$. A wavelength of $254 \mathrm{~nm}$ was used and the column was stabilized according to the initial conditions of the gradient. A $0.5 \mathrm{~mL} / \mathrm{min}$ flow rate was used with a $5 \mu \mathrm{L}$ injection volume at ambient temperature. Both compounds showed good retention and are well separated on the silica hydride column. 2,6-Diaminopurine being more polar than 1,2,4,5-benzenetetramine tetrahydrochloride eluted at 10.65 minutes and 1,2,4,5-benzenetetramine tetrahydrochloride eluted at 3.39 minutes.

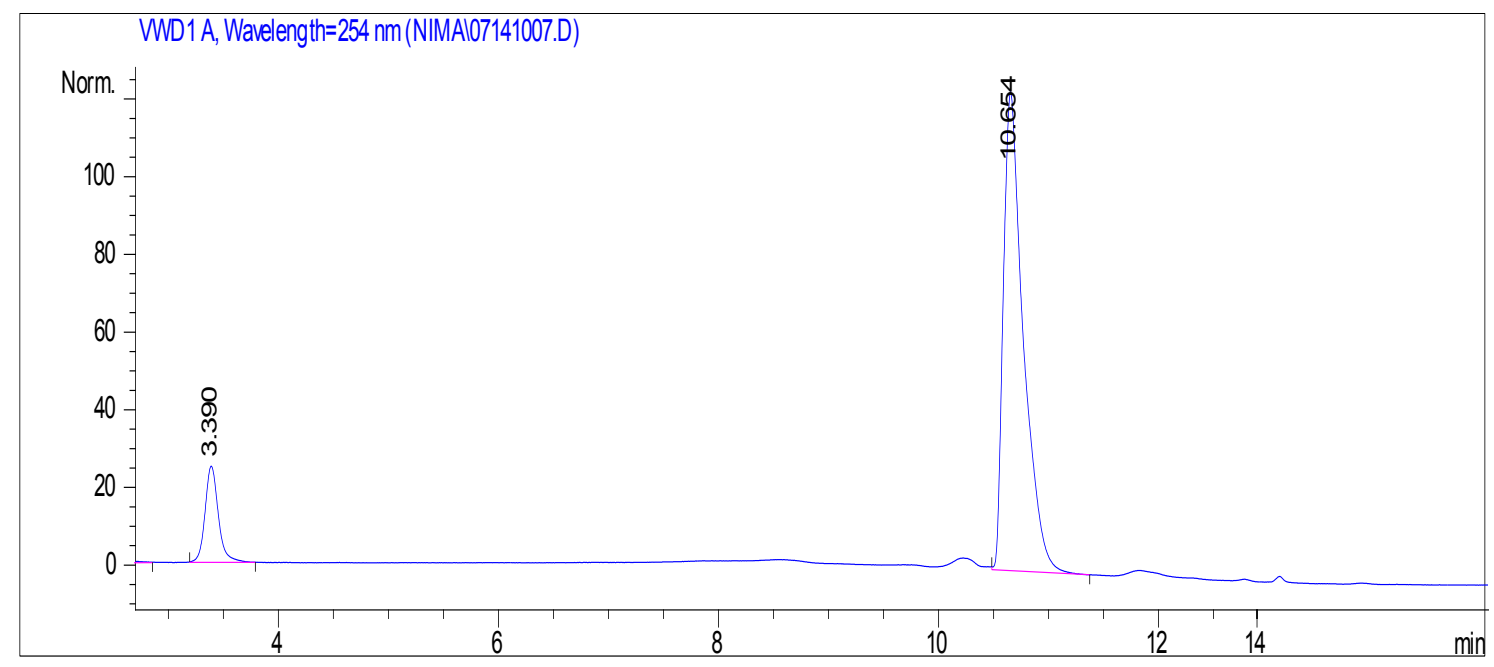

Figure 35 . Aqueous normal phase gradient separation of 1,2,4,5benzenetetramine tetrahydrochloride and 2,6-diaminopurine on the silica hydride column. The peak at 3.39 min is for 1,2,4,5-benzenetetramine tetrahydrochloride and the peak at 10.65 min is for 2,6-diaminopurine. Mobile phase Gradient A. 
The mixture of 2-phenethylamine and 2,6-diaminopurine was separated on the silica hydride column using aqueous normal phase conditions as shown in Figure 36 . The flow rate was $0.5 \mathrm{~mL} / \mathrm{min}$ with UV detection at $254 \mathrm{~nm}$. Gradient B described in Table 8 was used for this analysis. 2-Phenethylamine being a less polar compared to 2,6-diaminopurine eluted at 4.46 minutes and 2,6diaminopurine eluted at 6.06 minutes. Both compounds showed good retention and are well separated on the silica hydride column.

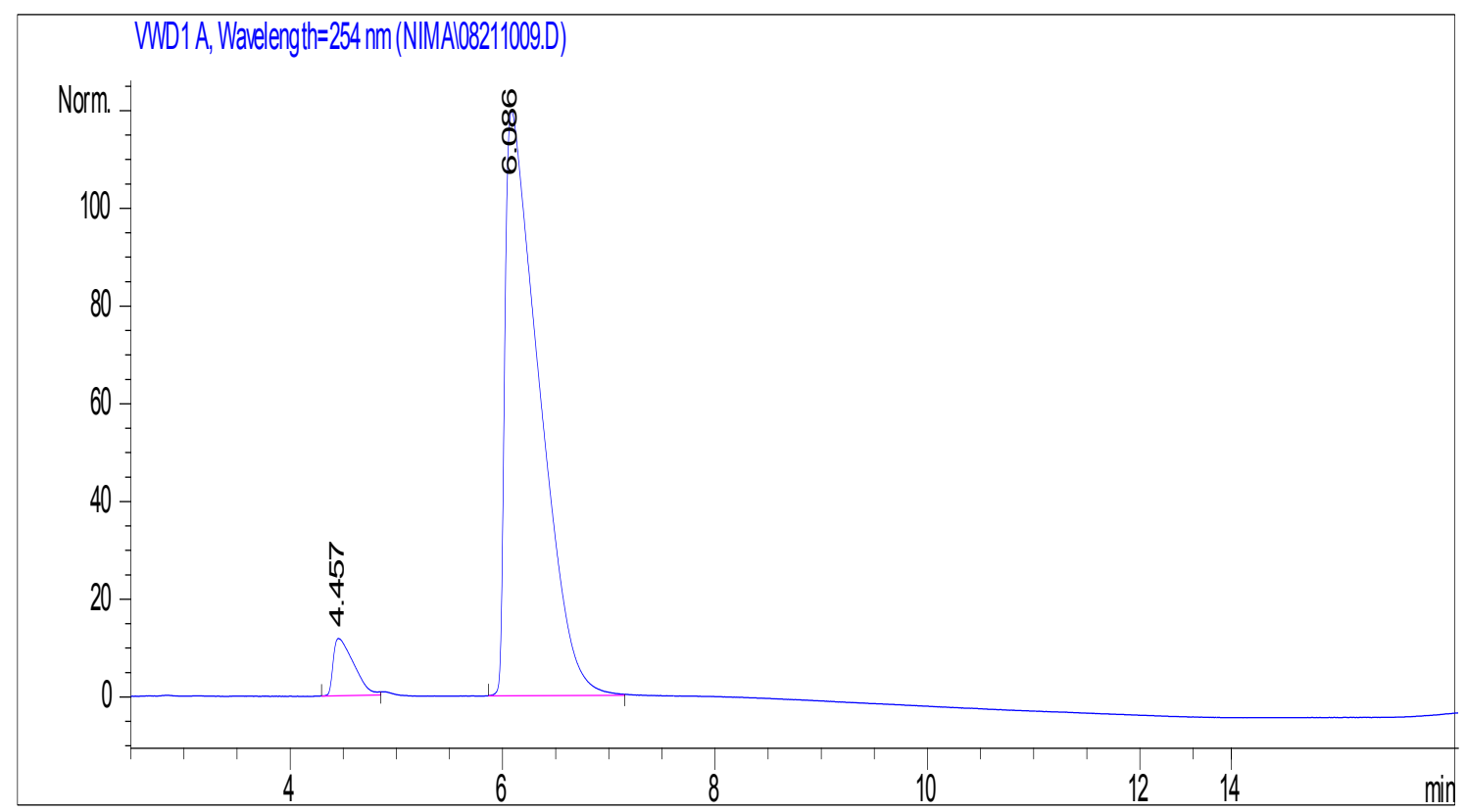

Figure 36. Aqueous normal phase gradient separation of 2-phenethylamine and 2,6-diaminopurine on the silica hydride column. The peak at $4.46 \mathrm{~min}$ is for 2phenethylamine and the peak at $6.08 \mathrm{~min}$ is for 2,6-diaminopurine.Mobile phase Gradient B.

The mixture of benzylamine and 2,6-diaminopurine was tested on the silica hydride column using aqueous normal phase conditions. The analysis was 
done using Gradient $\mathrm{D}$ at a mobile phase flow rate of $0.5 \mathrm{~mL} / \mathrm{min}$ and $\mathrm{UV}$ detection at $254 \mathrm{~nm}$. The chromatogram of the separation of benzylamine and 2,6-diaminopurine on the silica hydride column is shown in Figure 37.

Benzylamine was eluted at 11.38 minutes followed by 2,6-diaminopurine which was eluted at 12.87 minutes. Benzylamine showed peak tailing which can be solved by optimizing the gradient conditions. Therefore more work is needed in order to improve the appearance of the peak. In general, the silica hydride column demonstrated the ability to separate mixtures of primary amines using different gradient programs.

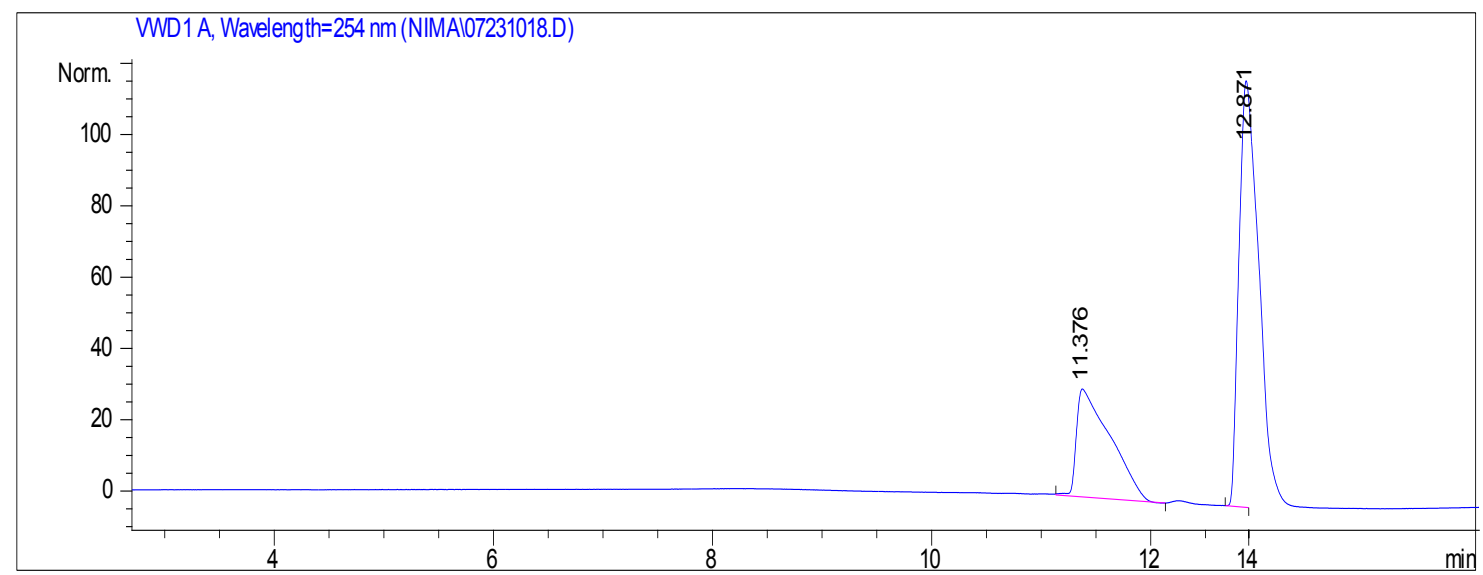

Figure 37. Aqueous normal phase gradient separation of benzylamine and 2,6diaminopurine on the silica hydride column. The peak at $11.38 \mathrm{~min}$ is for benzylamine and the peak at $12.87 \mathrm{~min}$ is for 2,6-diaminopurine. Mobile phase Gradient D.

\section{2. $\quad$ ANP Separation of Three Compounds Using Gradient Method}

A mixture of three primary amines was tested on the silica hydride column under aqueous normal phase gradient conditions. After confirming the 
retention of all primary amines on the silica hydride column using different gradient programs, a mixture of three compounds was tested. The gradient programs used for this study are shown in Table 8 . The flow rate was set at $0.5 \mathrm{~mL} / \mathrm{min}$ and a $5 \mu \mathrm{L}$ injection volume was used. The detector used for this analysis was UV at a wavelength of $254 \mathrm{~nm}$. All compounds were dissolved in $50: 50$ acetonitrile $(0.1 \%$ formic acid $) /$ water $(0.1 \%$ formic acid $)$ at a concentration of $0.5 \mathrm{mg} / \mathrm{mL}$. All the separations were performed at ambient temperature. The main goal of this part of the research was to analyze the behavior of the silica hydride stationary phase in the ANP mode and the following discussion will provide some details regarding this goal.

In Figure 38, the chromatogram shows the separation of 1,2,4,5benzenetetramine tetrahydrochloride, benzylamine and 2,6-diaminopurine. This analysis was carried out using Gradient A shown in Table 8. It can be seen from the chromatogram that 1,2,4,5-benzenetetramine tetrahydrochloride eluted first at 3.42 minutes because it is the least polar compared to the other two compounds. 2,6-Diaminopurine is the most polar of all three compounds. It eluted at 10.94 minutes and benzylamine eluted at 9.52 minutes. Benzylamine showed peak tailing which can be solved by optimizing the gradient conditions. Therefore, more work is needed in order to improve the appearance of the peaks in the chromatogram. 


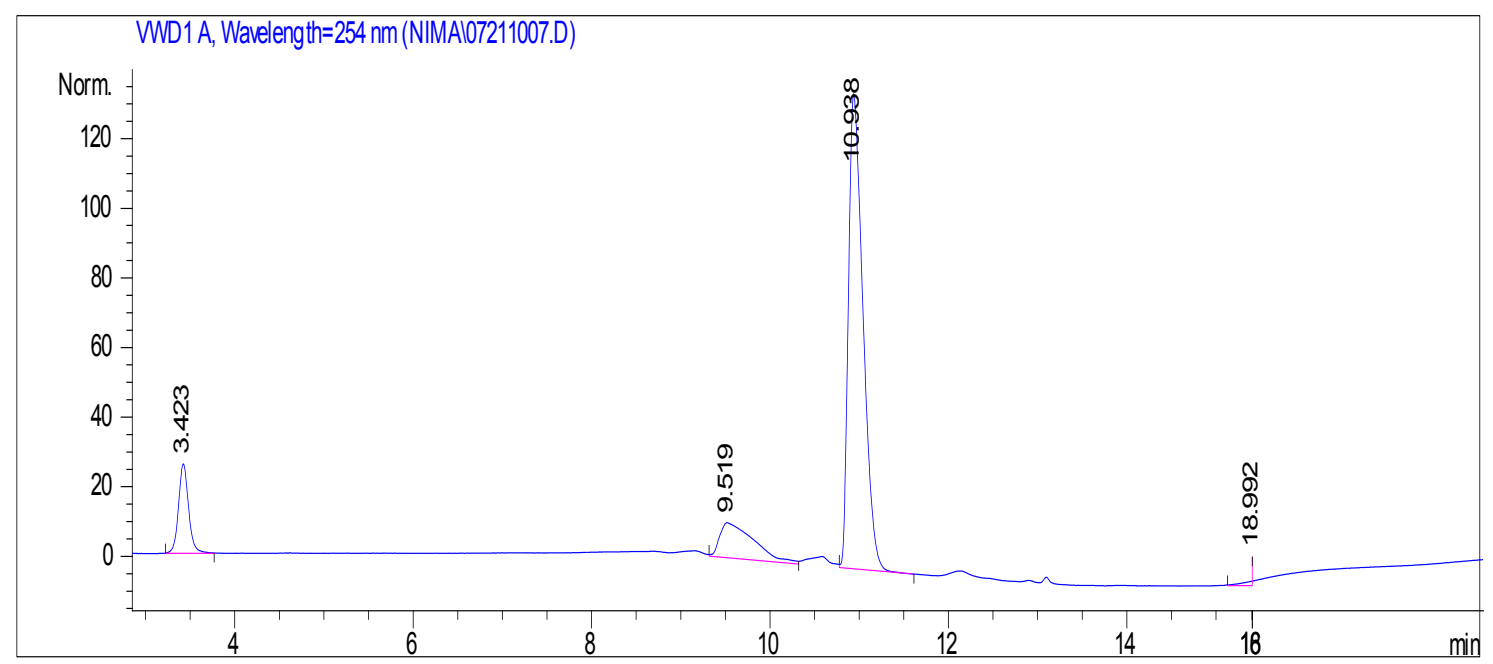

Figure 38. Aqueous normal phase gradient separation of 1,2,4,5benzenetetramine tetrahydrochloride, benzylamine and 2,6-diaminopurine on the silica hydride column. The peak at $3.42 \mathrm{~min}$ is for 1,2,4,5-benzenetetramine tetrahydrochloride, peak at $9.52 \mathrm{~min}$ is for benzylamine and the peak at 10.94 min is for 2,6-diaminopurine. Mobile phase Gradient A.

Another mixture of 1,2,4,5-benzenetetramine tetrahydrochloride, 2phenethylamine and 2,6-dimainopurine was tested on the silica hydride column under aqueous normal phase conditions as shown in Figure 39. The analysis was carried out using Gradient A at ambient temperature. It can be seen from the figure that the silica hydride column has successfully separated all three compounds. 1,2,4,5-Benzenetetramine tetrahydrochloride being the least polar compared to the other two eluted first at 3.41 minutes whereas 2-phenethylamine eluted at 9.33 minutes. 2,6-Diaminopurine showed longer retention on the column and eluted at 10.84 minutes. The peak tailing of 2-phenethylamine can be solved by optimizing the gradient conditions. The results are comparable with the diamond hydride column data which will be discussed in a later section. 


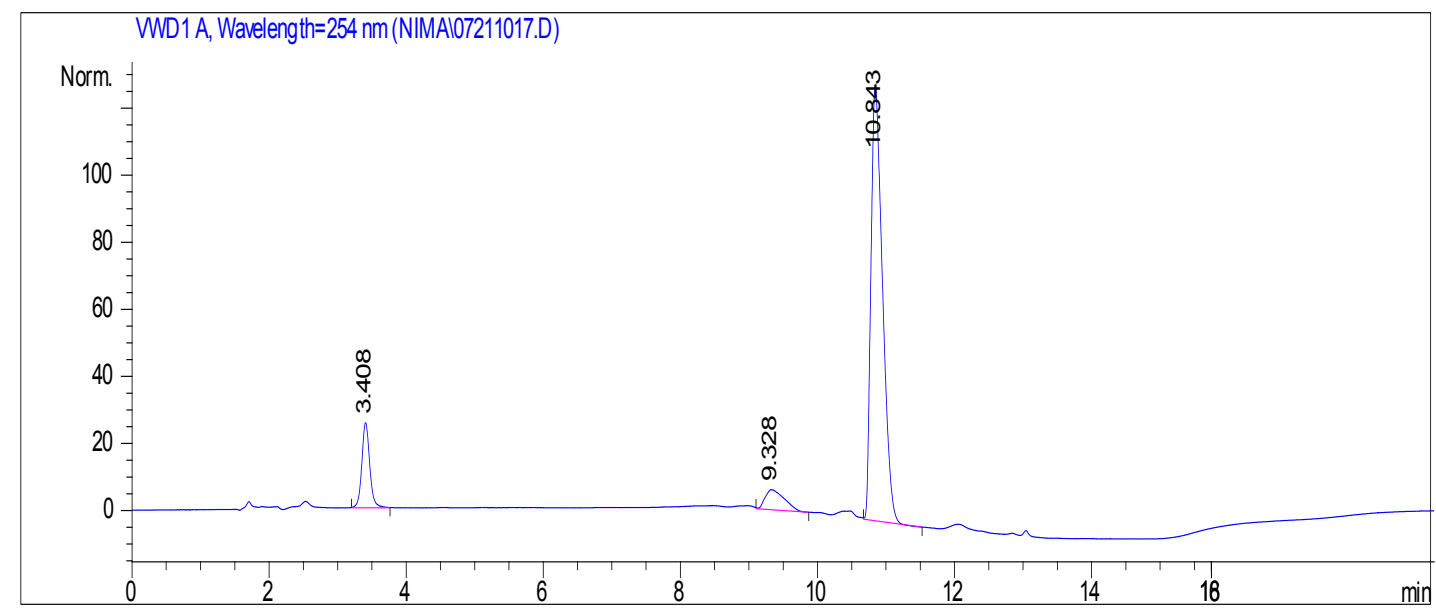

Figure 39. Aqueous normal phase gradient separation of 1,2,4,5benzenetetramine tetrahydrochloride, 2-phenethylamine and 2,6-diaminopurine on the silica hydride column. The peak at $3.41 \mathrm{~min}$ is for $1,2,4,5-$ benzenetetramine tetrahydrochloride, peak at $9.33 \mathrm{~min}$ is for 2-phenethylamine and the peak at $10.84 \mathrm{~min}$ is for 2,6-diaminopurine. Mobile phase Gradient A.

\section{3. $\quad$ ANP Separation of Four Compounds Using Gradient Method}

The separation of 1,2,4,5-benzenetetramine tetrahydrochloride, benzylamine, 2-phenethylamine and 2,6-diaminopurine is shown in Figure 40. After confirming their retention on the column using gradient conditions, a mixture of the four compounds was tested on the silica hydride column. This analysis was carried out using ANP gradient conditions on the silica hydride column with the UV detector. The separation was operated using Gradient $\mathrm{C}$ as shown in Table 9. The first compound to elute was 1,2,4,5-benzenetetramine tetrahydrochloride at 3.42 minutes showing the least retention on the column. Two of the compounds, 2-phenethylamine and benzylamine, did not separate and eluted as one single broad peak at 9.97 minutes. 2,6-Diaminopurine was the last compound to elute from the column at 11.27 minutes which showed the 
highest retention on the silica hydride column. Different gradient conditions were tried in order to improve the separation of these four compounds in mixtures but no significant improvement was obtained (data not shown here).

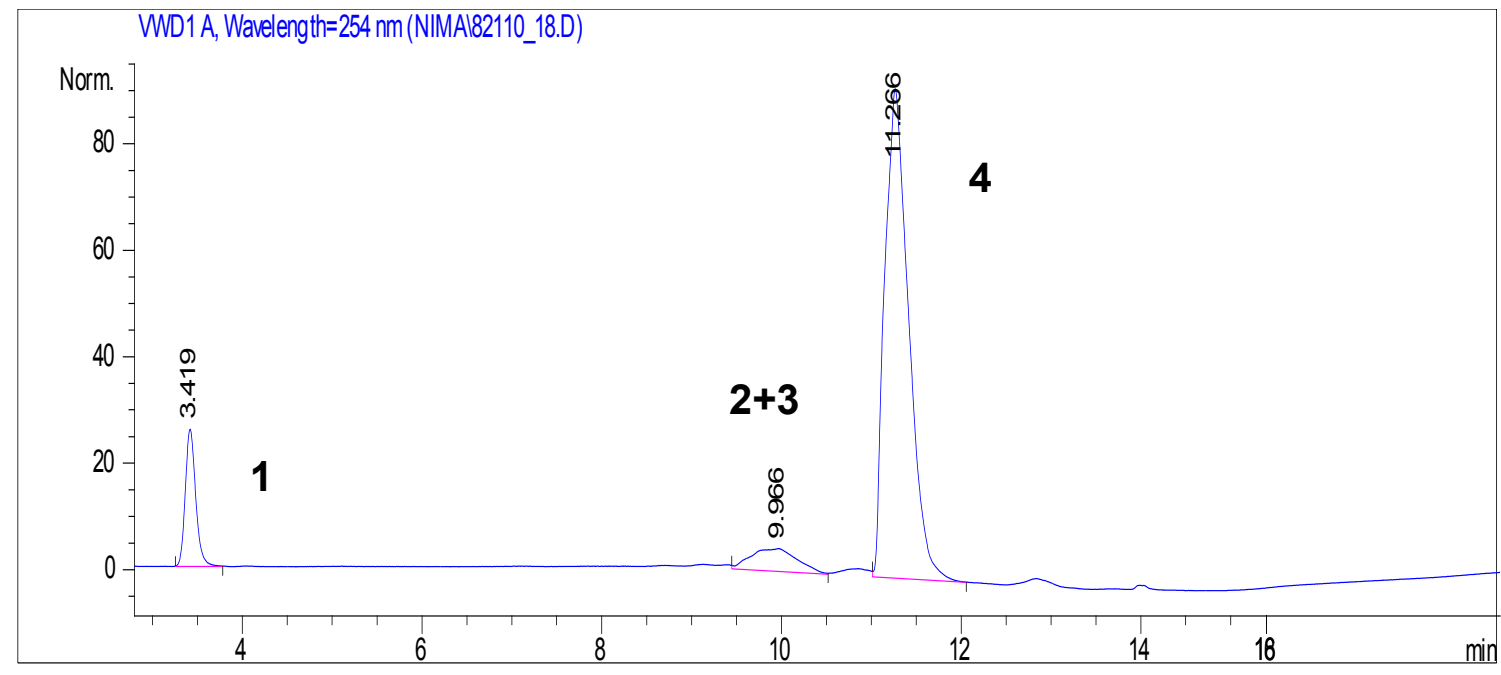

Figure 40. Aqueous normal phase gradient separation of 1,2,4,5benzenetetramine tetrahydrochloride, benzylamine, 2-phenethylamine and 2,6diaminopurine on the silica hydride column. Peak identification: $1=1,2,4,5-$ benzenetetramine tetrahydrochloride, peak $2+3=$ benzylamine and 2phenethylamine, peak 4 = 2,6-diaminopurine. Mobile phase Gradient A.

\subsection{ANP Behavior of the Diamond hydride Column Using the ELSD Detector}

The main goal of this work was to characterize the aqueous normal phase behavior of the diamond hydride column in the isocratic and gradient modes. An ELSD detector was used for the first part of this evaluation. The ELSD parameters were adjusted and stabilized according to the initial conditions of the isocratic run. To get a smooth baseline, the temperature of the drift tube 
and spray chamber were adjusted. The temperature of drift tube was raised to $65^{\circ} \mathrm{C}$ to evaporate the mobile phase whereas the temperature of the spray chamber was maintained at ambient temperature $\left(25^{\circ} \mathrm{C}\right)$. The flow rate was set at $0.5 \mathrm{~mL} / \mathrm{min}$ with a $20 \mu \mathrm{L}$ injection volume for most analyses. At the start of each day, a water blank run was performed to remove any contamination from the previous run.

\subsection{ANP Behavior of Adenosine}

Adenosine was analyzed in the isocratic mode using an ELSD detector to study its retention characteristics on the diamond hydride column. The concentration of adenosine was $1 \mathrm{mg} / \mathrm{mL}$ in $50: 50$ acetonitrile/water (both premixed with $0.1 \%$ formic acid). A water blank was run before each HPLC run to remove any contamination from the previous run. The ELSD parameters were adjusted and stabilized according to the initial conditions of the isocratic test. The mobile phase flow rate was set at $0.5 \mathrm{~mL} / \mathrm{min}$ at ambient temperature with a $20 \mu \mathrm{L}$ injection volume.

Aqueous normal phase conditions were used to analyze adenosine on the diamond hydride column. The mobile phase composition used was acetonitrile with $0.1 \%$ formic acid and water with $0.1 \%$ formic acid. A series of tests were carried out in the isocratic mode with the composition of acetonitrile ( $0.1 \%$ formic acid) in the mobile phase varied from $50 \%$ to $90 \%$. Adenosine was well retained on the diamond hydride column under all conditions. 
Chromatograms of different mobile phase compositions are shown in Figure 41. It can be seen from Table 19 that adenosine showed aqueous normal phase behavior as retention increased with an increase of the organic component in the mobile phase. Figure 42 confirms the ANP retention of adenosine on the diamond hydride column.
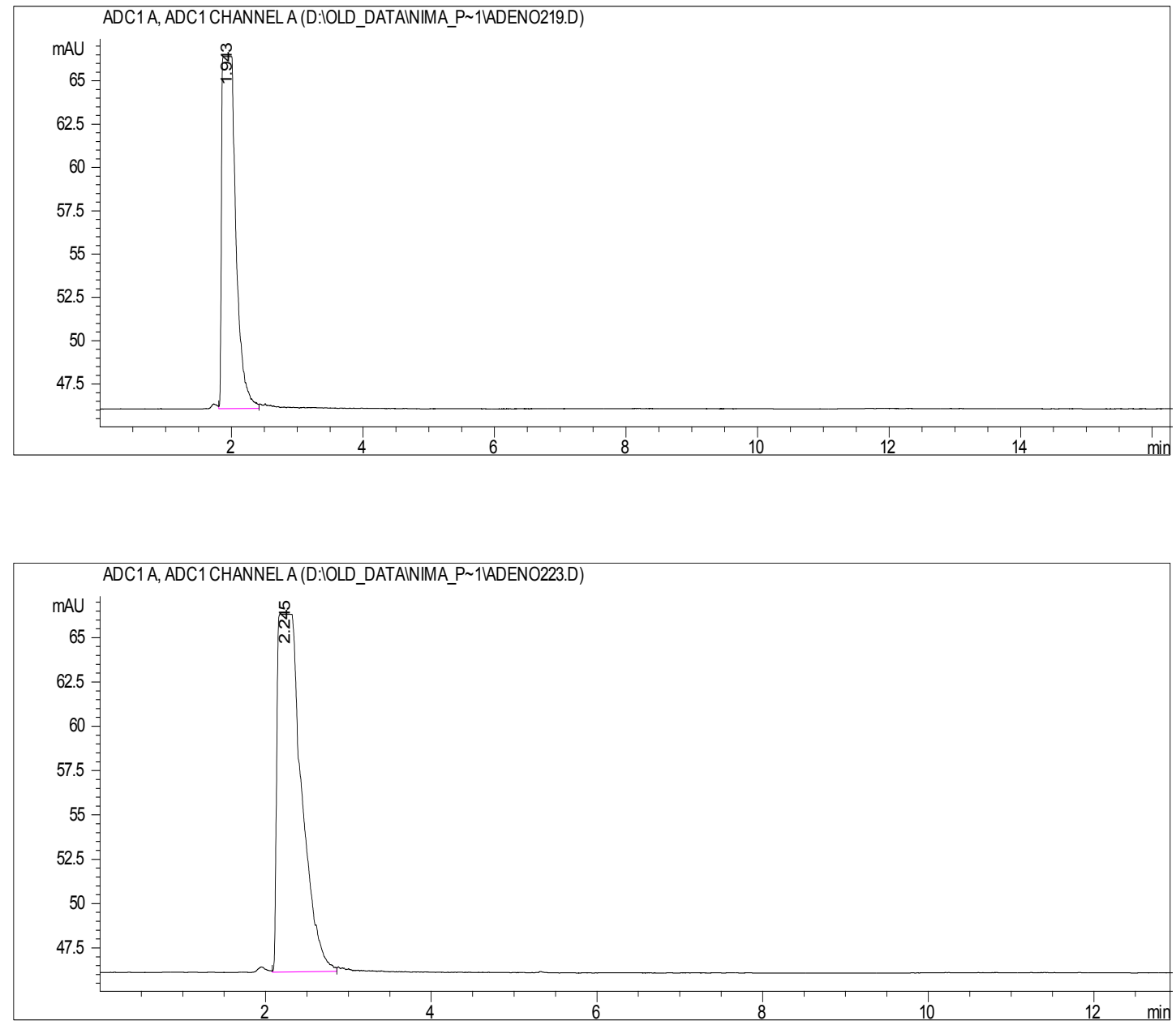

Figure 41. Chromatograms of adenosine obtained using the diamond hydride stationary phase at 50:50 acetonitrile/water (Top) and 80:20 acetonitrile/water (Bottom) mobile phase (both premixed with $0.1 \%$ formic acid). 
Table 19. Retention time of adenosine on the diamond hydride column using ELSD.

\begin{tabular}{|c|c|c|}
\hline \multicolumn{2}{|c|}{ Concentration (\%) } & Retention time(min) \\
\hline $\begin{array}{c}\text { Acetonitrile with 0.1\% } \\
\text { FA }\end{array}$ & DI water with 0.1\% FA & Adenosine \\
\hline 50 & 50 & 1.94 \\
\hline 60 & 40 & 1.97 \\
\hline 70 & 30 & 2.0 \\
\hline 80 & 20 & 2.74 \\
\hline
\end{tabular}

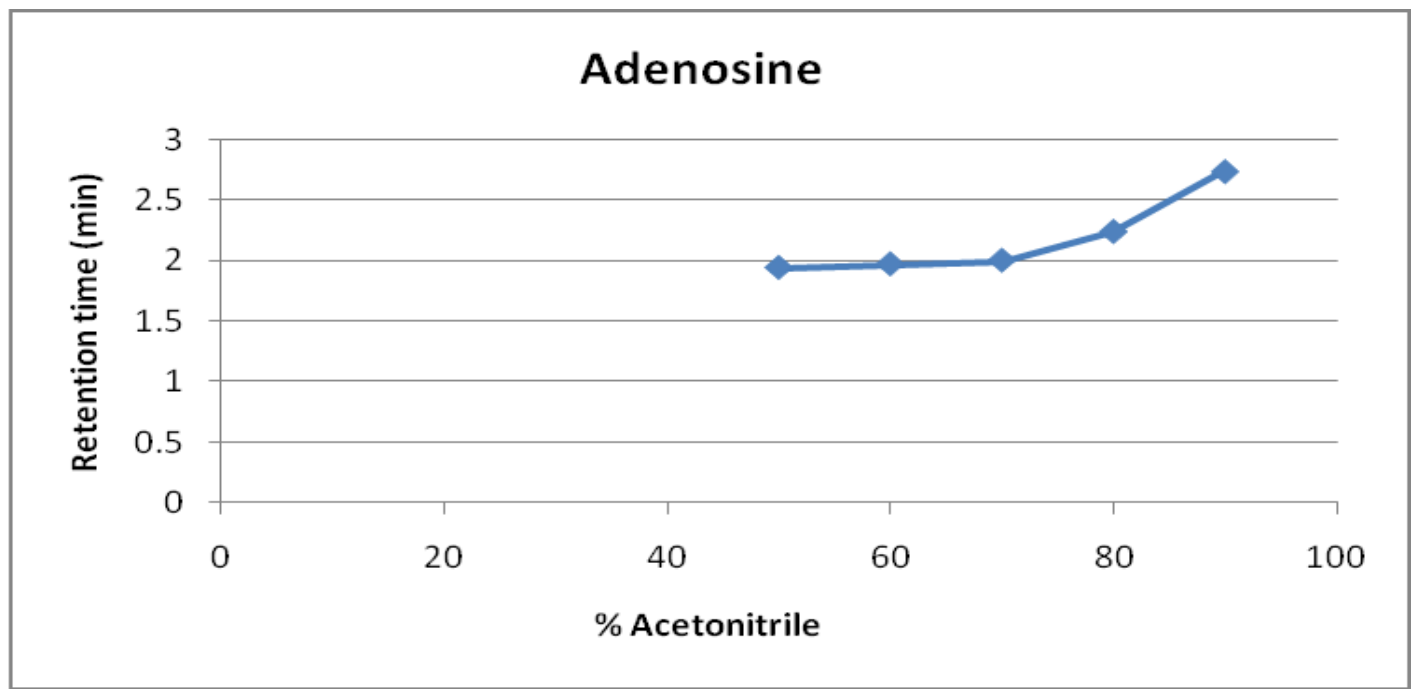

Figure 42. Retention map of adenosine on the diamond hydride column using ELSD

\subsection{ANP Behavior of Guanine}

ANP isocratic conditions were used to analyze guanine on the diamond hydride column. Guanine was difficult to dissolve so $200 \mu \mathrm{L} \mathrm{HCl}$ solution was mixed with 50:50 acetonitrile/water (both premixed with $0.1 \%$ formic acid) to prepare a $1 \mathrm{mg} / \mathrm{mL}$ sample solution. The ELSD parameters were adjusted and 
stabilized according to the starting conditions of the isocratic test. Guanine was analyzed under aqueous normal phase conditions as shown in the chromatograms of Figure 43 . The mobile phase used was acetonitrile $(0.1 \%$ formic acid) and water ( $0.1 \%$ formic acid).
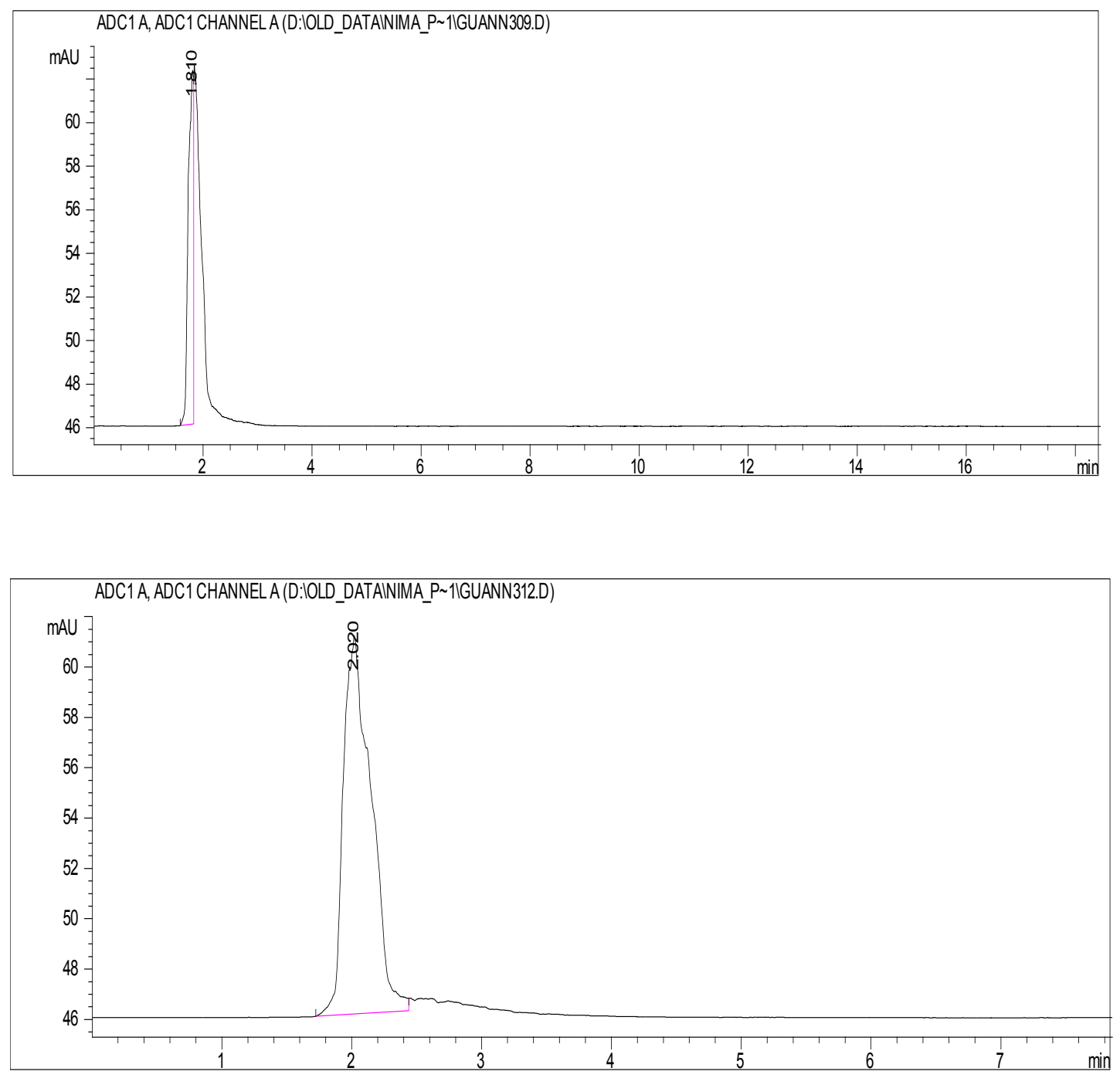

Figure 43. Chromatograms of guanine obtained using the diamond hydride stationary phase at 70:30 acetonitrile/water (Top) and 80:20 acetonitrile/water (Bottom) mobile phase (both premixed with $0.1 \%$ formic acid). 
Retention times of guanine for various compositions of mobile phase are shown in Table 20. It can be seen from the retention map of guanine in Figure 44, that guanine was retained using the aqueous normal phase elution mode. In the aqueous normal phase mode, as the composition of acetonitrile ( $0.1 \%$ formic acid) was increased in the mobile phase, the retention time of guanine also increased. This indicated the ANP behavior of guanine on the diamond hydride column.

Table 20. Retention time of guanine on the diamond hydride using ELSD.

\begin{tabular}{|c|c|c|}
\hline \multicolumn{2}{|c|}{ Concentration (\%) } & Retention time (min) \\
\hline $\begin{array}{c}\text { Acetonitrile with 0.1\% } \\
\text { FA }\end{array}$ & DI water with 0.1\% FA & Guanine \\
\hline 50 & 50 & 1.69 \\
\hline 60 & 40 & 1.74 \\
\hline 70 & 30 & 1.81 \\
\hline 80 & & 2.02 \\
\hline 90 & 20 & \\
\hline & & \\
\hline
\end{tabular}




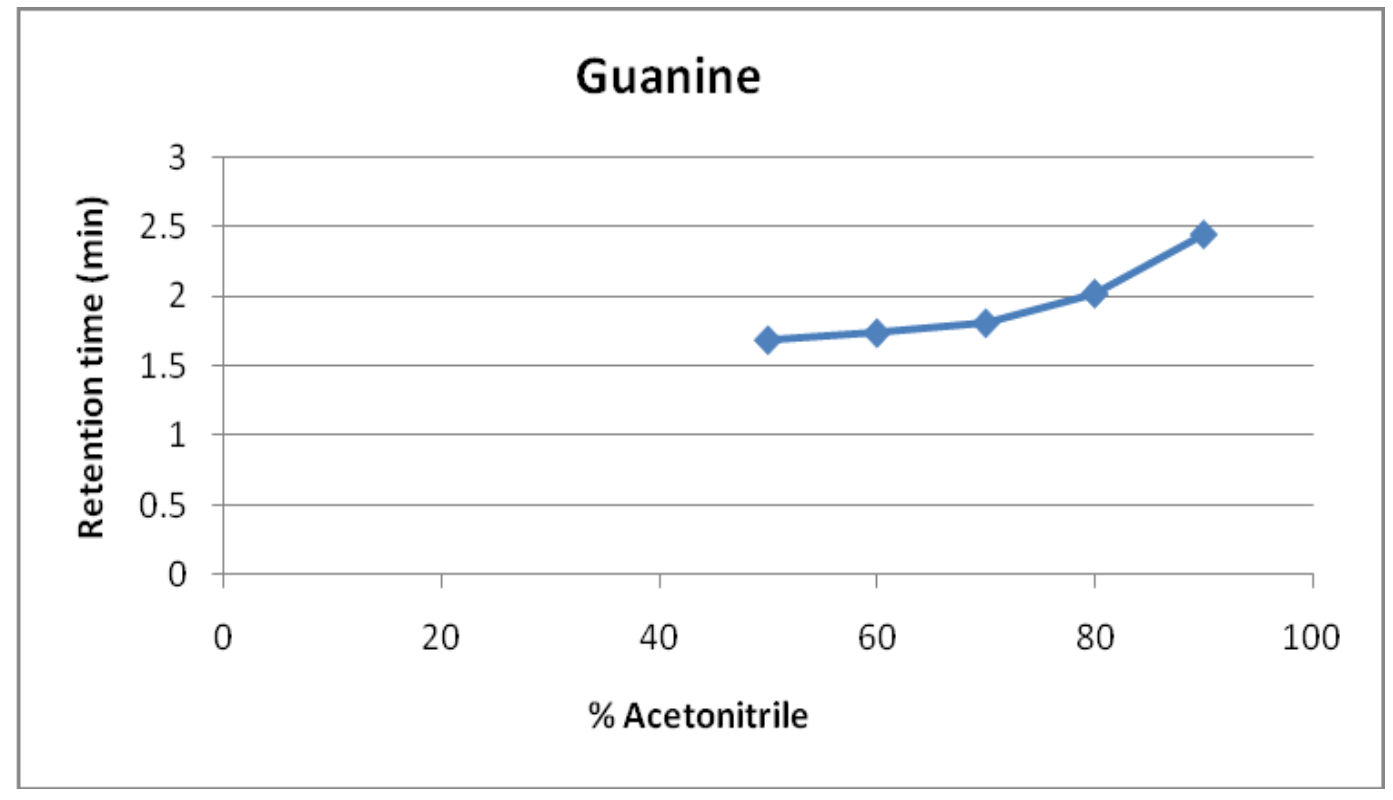

Figure 44. Retention map of guanine on the diamond hydride column using ELSD.

\subsection{ANP Behavior of Creatinine}

The analysis of creatinine was carried out under ANP isocratic conditions on the diamond hydride column. Creatinine was dissolved in 50:50 acetonitrile/water (both premixed with $0.1 \%$ formic acid) at a concentration of $1 \mathrm{mg} / \mathrm{mL}$. A $0.5 \mu \mathrm{L} / \mathrm{min}$ flow rate was set with a $20 \mu \mathrm{L}$ injection volume. ELSD parameters were set and stabilized with the starting conditions of isocratic test. Various ratios of acetonitrile/water (both premixed with $0.1 \%$ formic acid), varied from $50 \%$ to $90 \%$ were used for the analysis. Chromatograms of creatinine for 50:50 and 80:20 acetonitrile/water (with $0.1 \%$ formic acid) are shown in Figure 45. The retention times of the sample at various concentrations of organic component in the mobile phase are listed in Table 21. It can be seen from Figure 46 that retention time of the compound gradually increased with a higher 
percentage of the organic component in the mobile phase which clearly indicates the ANP behavior of creatinine on the diamond hydride column. The retention map of creatinine is shown in Figure 46.
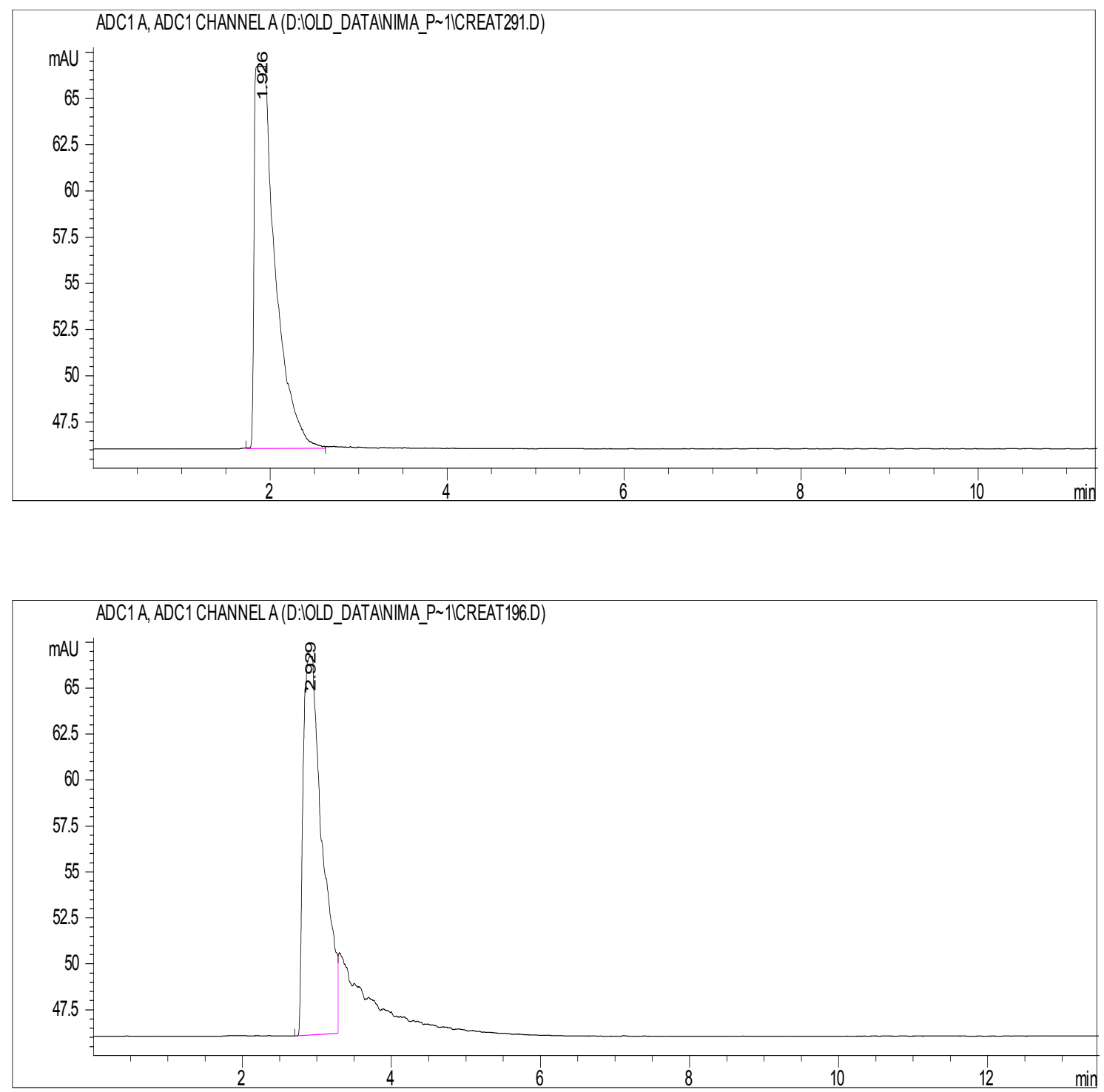

Figure 45. Chromatograms of creatinine obtained using the diamond hydride stationary phase at 50:50 acetonitrile/water (Top) and 80:20 acetonitrile/water (Bottom) mobile phase (both premixed with $0.1 \%$ formic acid). 
Table 21. Retention time of creatinine on the diamond hydride column using ELSD.

\begin{tabular}{|c|c|c|}
\hline \multicolumn{2}{|c|}{ Concentration (\%) } & Retention time (min) \\
\hline $\begin{array}{c}\text { Acetonitrile with 0.1\% } \\
\text { FA }\end{array}$ & DI water with 0.1\% FA & Creatinine \\
\hline 50 & 50 & 1.92 \\
\hline 60 & 40 & 1.94 \\
\hline 70 & 30 & 2.26 \\
\hline 80 & 20 & 2.93 \\
\hline 90 & 10 & 3.03 \\
\hline
\end{tabular}

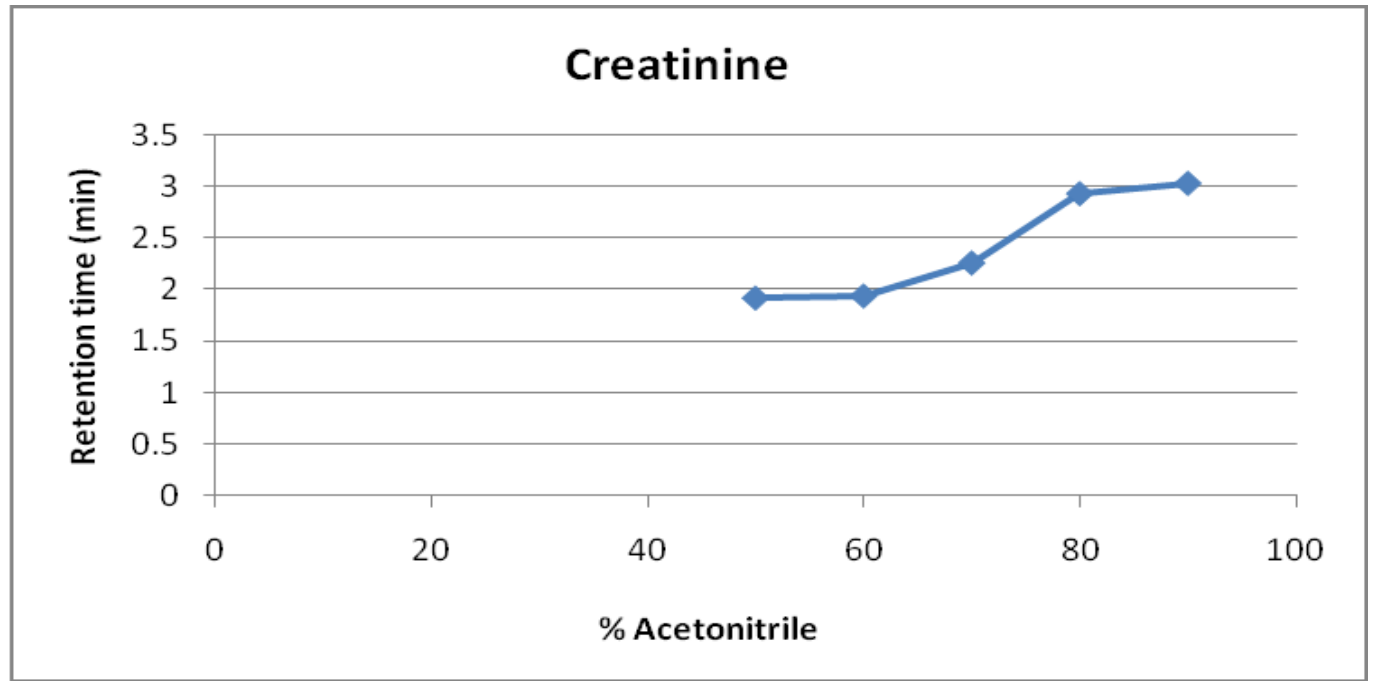

Figure 46. Retention map of creatinine on the diamond hydride column using ELSD. 


\subsection{ANP Behavior of Guanidine}

Guanidine was analyzed in the ANP isocratic mode on the diamond hydride column using an ELSD detector. The concentration of guanidine used for the analysis was $1 \mathrm{mg} / \mathrm{mL}$. The mobile phase used was acetonitrile/water (both premixed with $0.1 \%$ formic acid) with a $0.5 \mathrm{~mL} / \mathrm{min}$ flow rate and a $20 \mu \mathrm{L}$ injection volume. The ELSD parameters were stabilized and set according to the starting conditions of the isocratic test. Table 22 presents the retention time of guanidine as the concentration of acetonitrile was increased from $50 \%$ to $90 \%$. Figure 47 shows the retention map of guanidine and confirms the typical ANP behavior of guanidine.

Table 22. Retention time of guanidine on the diamond hydride column using ELSD.

\begin{tabular}{|c|c|c|}
\hline \multicolumn{2}{|c|}{ Concentration (\%) } & Retention time (min) \\
\hline $\begin{array}{c}\text { Acetonitrile with 0.1\% } \\
\text { FA }\end{array}$ & DI water with 0.1\% FA & Guanidine \\
\hline 50 & 50 & 1.91 \\
\hline 60 & 40 & 2.08 \\
\hline 70 & 30 & 2.41 \\
\hline 80 & 20 & 2.49 \\
\hline 90 & 10 & 3.51 \\
\hline
\end{tabular}




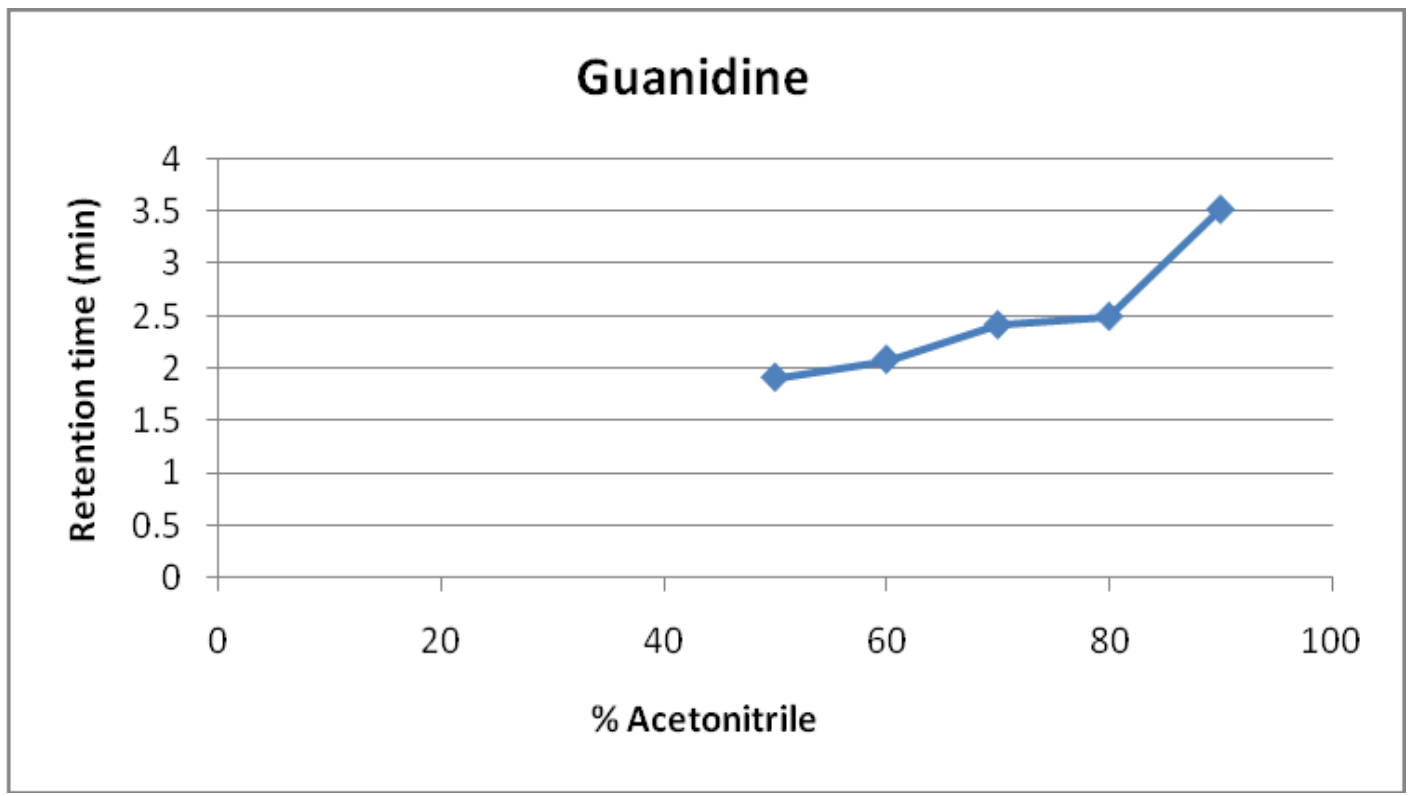

Figure 47. Retention map of guanidine on the diamond hydride column using ELSD.

\section{5. $\quad$ ANP Behavior of L-Tryptophan}

L-Tryptophan was analyzed in the ANP isocratic mode on the diamond hydride column using an ELSD detector. The concentration of L-tryptophan used for the analysis was $1 \mathrm{mg} / \mathrm{mL}$. The mobile phase used was acetonitrile/water (both premixed with $0.1 \%$ formic acid) with a $0.5 \mathrm{~mL} / \mathrm{min}$ flow rate and a $20 \mu \mathrm{L}$ injection volume. The ELSD parameters were stabilized and set according to the starting conditions of the isocratic test.

Table 23 lists the retention time of L-tryptophan as the concentration of acetonirile increases from $50 \%$ to $90 \%$. Figure 48 shows the retention map of Ltryptophan at various $\%$ of acetonitrile. This data confirms a typical ANP behavior of L-tryptophan on the diamond hydride column. 
Table 23. Retention time of L-tryptophan on the diamond hydride column using ELSD.

\begin{tabular}{|c|c|c|}
\hline \multicolumn{2}{|c|}{ Concentration (\%) } & Retention time (min) \\
\hline $\begin{array}{c}\text { Acetonitrile with 0.1\% } \\
\text { FA }\end{array}$ & DI water with0.1\% FA & L-Tryptophan \\
\hline 50 & 50 & 1.94 \\
\hline 60 & 40 & 2.11 \\
\hline 70 & 30 & 2.29 \\
\hline 80 & 20 & 3.04 \\
\hline 90 & 10 & 3.11 \\
\hline
\end{tabular}

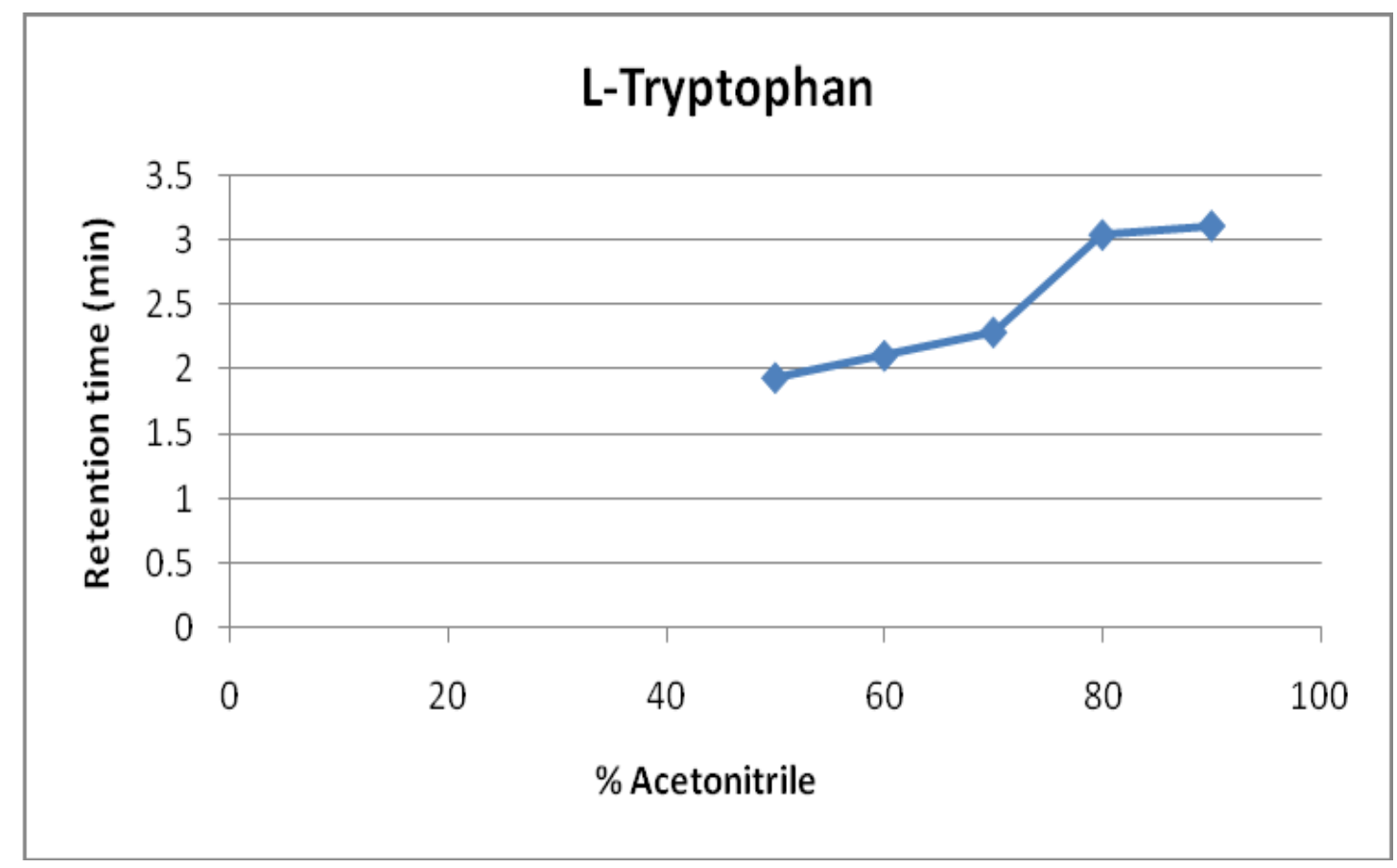

Figure 48. Retention map of L-tryptophan on the diamond hydride column using ELSD. 


\subsection{ANP Behavior of Diamond hydride Column Using a UV Detector}

As stated earlier, because of the further unavailability of the ELSD detector the emphasis of HPLC separations for the remaining research was shifted to the UV detector for remaining ANP retention study of the diamond hydride column. Different classes of compounds e.g. primary amines, nucleobases, ergogenic acids and nucleosides were analyzed to establish their characteristic ANP retention. Primary amines were tested under isocratic as well as gradient conditions. The characterization of the diamond hydride column was done in the aqueous normal phase mode. The flow rate was set at $0.5 \mathrm{~mL} / \mathrm{min}$, at ambient temperature with a $5 \mu \mathrm{L}$ injection volume. The concentration of the samples used for the analysis was $0.5 \mathrm{mg} / \mathrm{mL}$ in $50: 50$ water/acetonitrile solvent (both premixed with $0.1 \%$ formic acid). A wavelength of $254 \mathrm{~nm}$ was selected for all analyses. The diamond hydride column was maintained at the starting conditions of the gradient or isocratic conditions until a stable baseline was obtained. A water blank was run to eliminate any contamination from the previous measurements. The gradient programs used for this research are shown in Table 8 and Table 9. The main goal of this research was to perform chromatographic characterization of the diamond hydride column under aqueous normal phase mode using isocratic and gradient methods. 


\subsection{ANP Behavior of Adenosine}

A nucleoside, adenosine was analyzed under ANP solvent conditions on the diamond hydride column. Adenosine was dissolved in 50:50 acetonitrile ( $0.1 \%$ formic acid) $/$ water $(0.1 \%$ formic acid) at a $0.5 \mathrm{mg} / \mathrm{mL}$ concentration. A UV wavelength of $254 \mathrm{~nm}$ was selected to monitor the peaks and stabilized according to the initial conditions of the isocratic test.

Figure 49 shows the chromatograms of adenosine for different mobile phase compostions which demonstrates the aqueous normal phase behavior of the compound. Figure 50 indicates that with an increasing percentage of acetonitrile $(0.1 \%$ formic acid $)$, the retention times for adenosine is increasing gradually. This behavior indicates the ANP behavior of adenosine on the diamond hydride column.

As can be seen from Table 24, adenosine showed almost similar retention times of 2.90 minutes and 2.94 minutes at $50: 50$ and $60: 40$ acetonitrile/water (with $0.1 \% \mathrm{FA}$ ) respectively. This almost constant retention of the compound for these two compositions may be a characteristic behavior of the diamond hydride column. In general, for 60:40 to 90:10 acetonitrile/water (with $0.1 \%$ FA) compositions, adenosine showed highly efficient symmetric peaks on the diamond hydride column. 

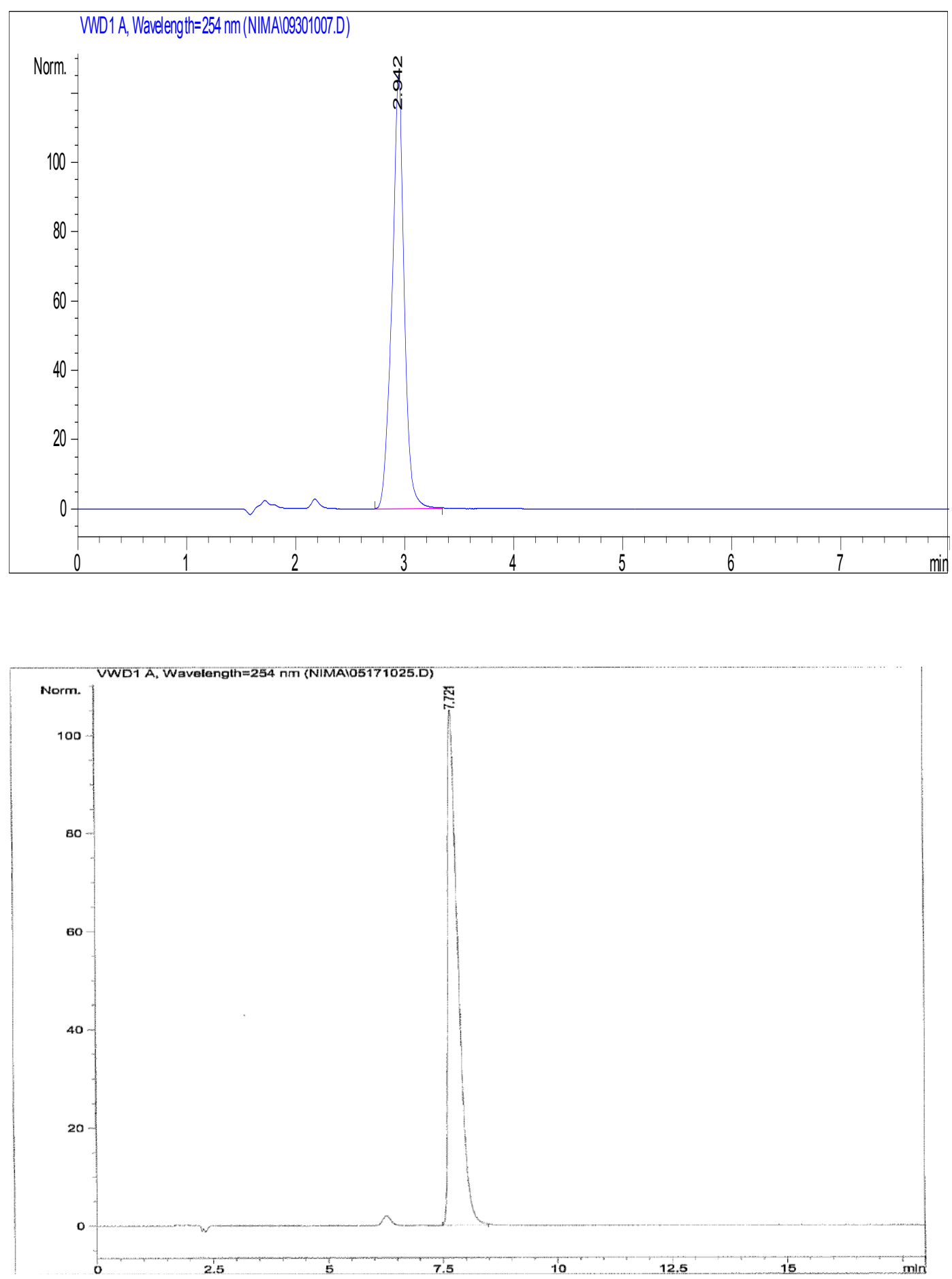

Figure 49. Chromatograms of adenosine obtained using the diamond hydride stationary phase at 60:40 acetonitrile/water (Top) and 90:10 acetonitrile/water (Bottom) mobile phase (both premixed with $0.1 \%$ formic acid). 
Table 24. Retention time of adenosine on the diamond hydride column using UV detector.

\begin{tabular}{|c|c|c|}
\hline \multicolumn{2}{|c|}{ Concentration (\%) } & Retention time (min) \\
\hline $\begin{array}{c}\text { Acetonitrile with 0.1\% } \\
\text { FA }\end{array}$ & DI water with 0.1\% FA & Adenosine \\
\hline 50 & 50 & 2.90 \\
\hline 60 & 40 & 2.94 \\
\hline 70 & 30 & 3.70 \\
\hline 80 & 20 & 4.75 \\
\hline 90 & 10 & 7.72 \\
\hline
\end{tabular}

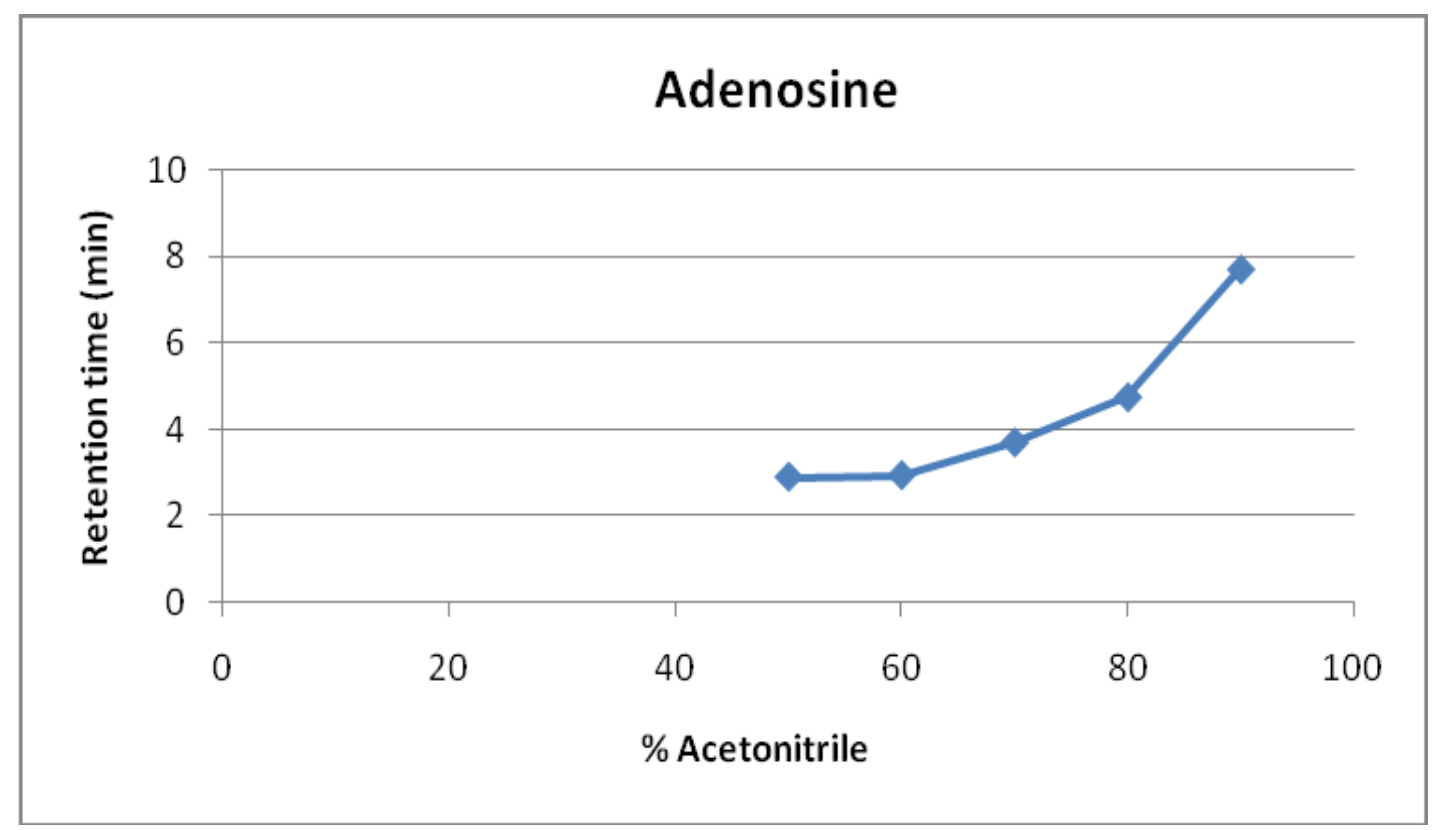

Figure 50. Retention map of adenosine on the diamond hydride column using UV detection. 


\subsection{ANP Behavior of Guanine}

Guanine was difficult to dissolve so $200 \mu \mathrm{L} \mathrm{HCl}$ solution was added in 50:50 acetonitrile/water (both premixed with $0.1 \%$ formic acid) to prepare a $0.5 \mathrm{mg} / \mathrm{mL}$ sample solution. The flow rate was set at $0.5 \mu \mathrm{L} / \mathrm{min}$ and an injection volume of $5 \mu \mathrm{L}$ was used. A wavelength of $254 \mathrm{~nm}$ UV was selected and the column was stabilized according to the starting conditions of the isocratic test. Guanine was analyzed using $0.1 \%$ formic acid in water and $0.1 \%$ formic acid in acetonitrile under ANP isocratic conditions on the diamond hydride column using a UV detector.

Table 25 shows the retention times of guanine at different mobile phase concentrations. Figure 51 shows the chromatograms of the compound at $60: 40$ and $80: 20$ acetonitrile/water (with $0.1 \%$ formic acid) compositions.

Table 25. Retention time of guanine on the diamond hydride column using UV detector.

\begin{tabular}{|c|c|c|}
\hline \multicolumn{2}{|c|}{ Concentration (\%) } & Retention time (min) \\
\hline $\begin{array}{c}\text { Acetonitrile with 0.1\% } \\
\text { FA }\end{array}$ & DI water with 0.1\% FA & Guanine \\
\hline 50 & 50 & 2.50 \\
\hline 60 & 40 & 2.51 \\
\hline 70 & 30 & 2.84 \\
\hline 80 & 20 & 3.59 \\
\hline 90 & 10 & 8.01 \\
\hline
\end{tabular}



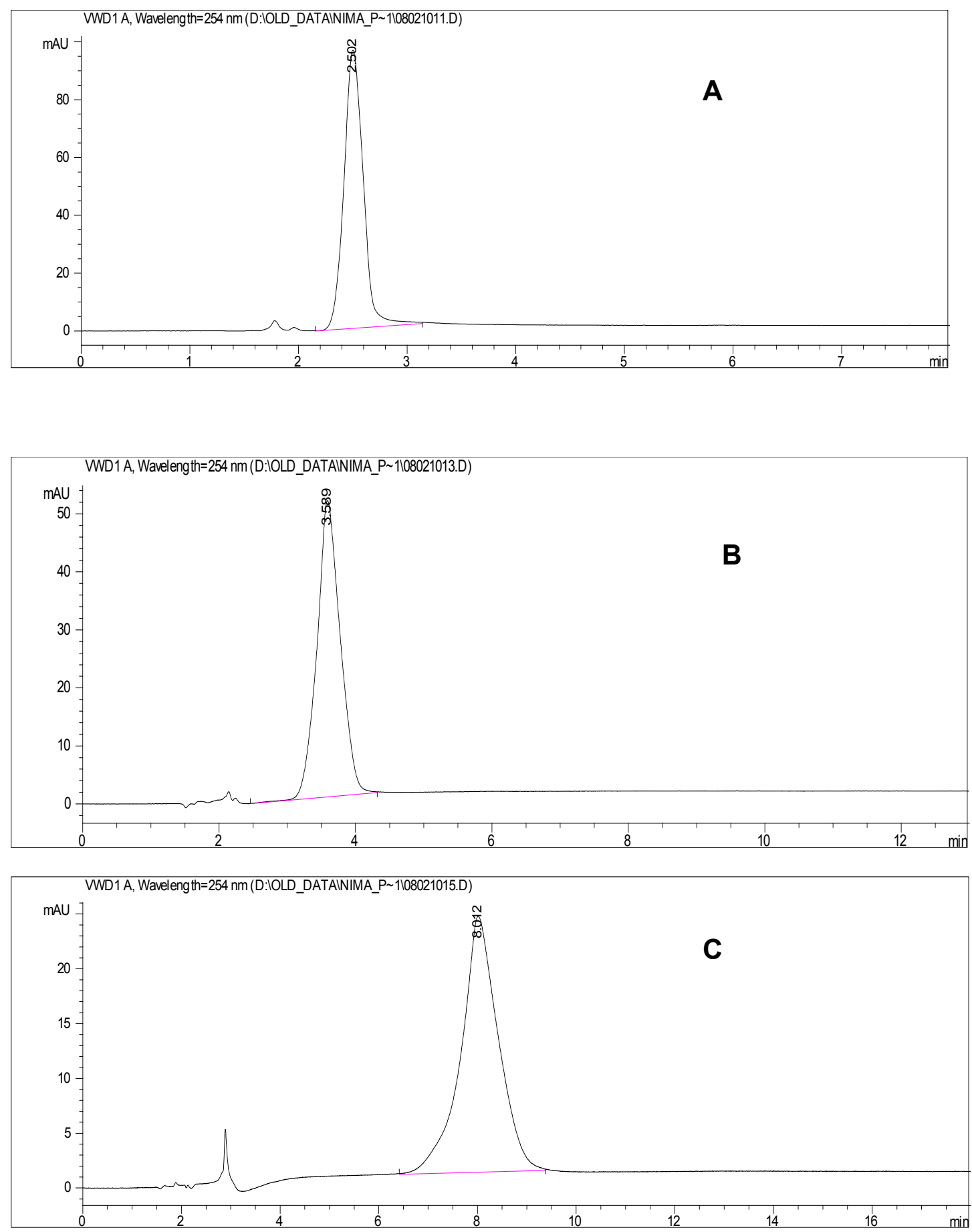

Figure 51. Chromatograms of guanine obtained on the diamond hydride stationary phase. A. 60:40 acetonitrile/water with 0.1\% FA. B. 80:20 acetonitrile/water with $0.1 \%$ FA. C. $90: 10$ acetonitrile/water mobile phase with $0.1 \% \mathrm{FA}$. 
Figure 52 is the retention map of guanine confirming the ANP behavior of this nucleobase on the diamond hydride column. The retention of guanine increases from 2.5 minutes at $50 \%$ acetonitrile to 8.01 minutes at $90 \%$ acetonitrile. This data confirms the ANP behavior of guanine on the diamond hydride column.

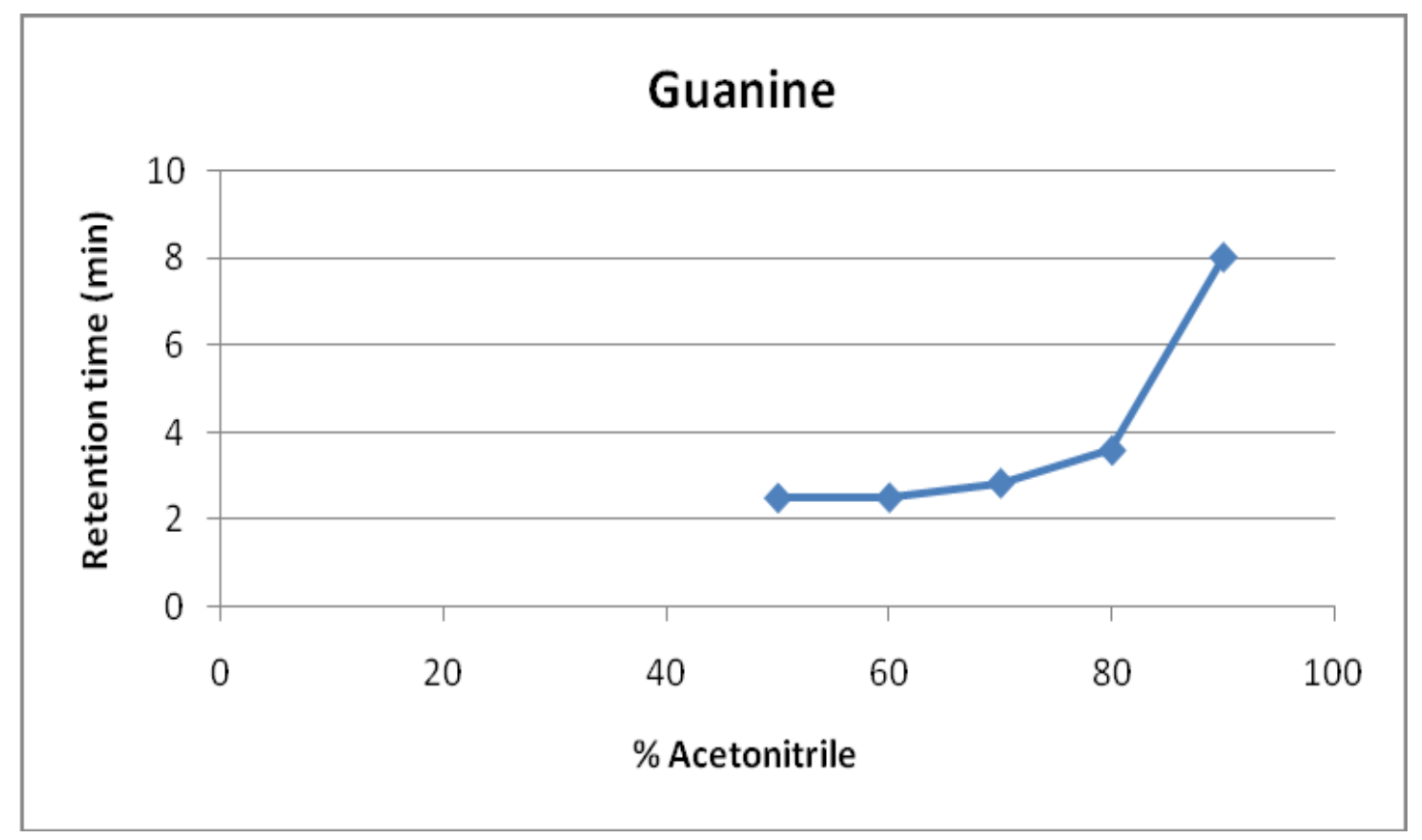

Figure 52. Retention map of guanine on the diamond hydride column using UV detection.

\subsection{ANP Behavior of L-Tryptophan}

L-Tryptophan was analyzed using ANP solvent conditions on the diamond hydride column with UV detection. The flow rate was set at $0.5 \mathrm{~mL} / \mathrm{min}$, and a UV absorption wavelength of $254 \mathrm{~nm}$ was selected. A sample solution at a concentration of $0.5 \mathrm{mg} / \mathrm{mL}$ was prepared using 50:50 acetonitrile/water (both 
premixed with $0.1 \%$ formic acid) mobile phase composition. The injection volume was set at $5 \mu \mathrm{L}$. All the runs were performed at ambient temperature.

Table 26 shows the retention time of L-tryptophan with increasing concentration of organic solvent from $50 \%$ to $90 \%$. It can be seen from the retention table that with the increase in percentage of acetonitrile, the retention time of L-tryptophan also increased. L-Tryptophan displayed high retention up to approximately 12 minutes for $90 \%$ acetonitrile on the diamond hydride column as shown in Figure 53. Figure 54 represents the characteristic ANP retention map of the compound. These observations indicate that the compound has been retained on the diamond hydride column in the ANP mode.

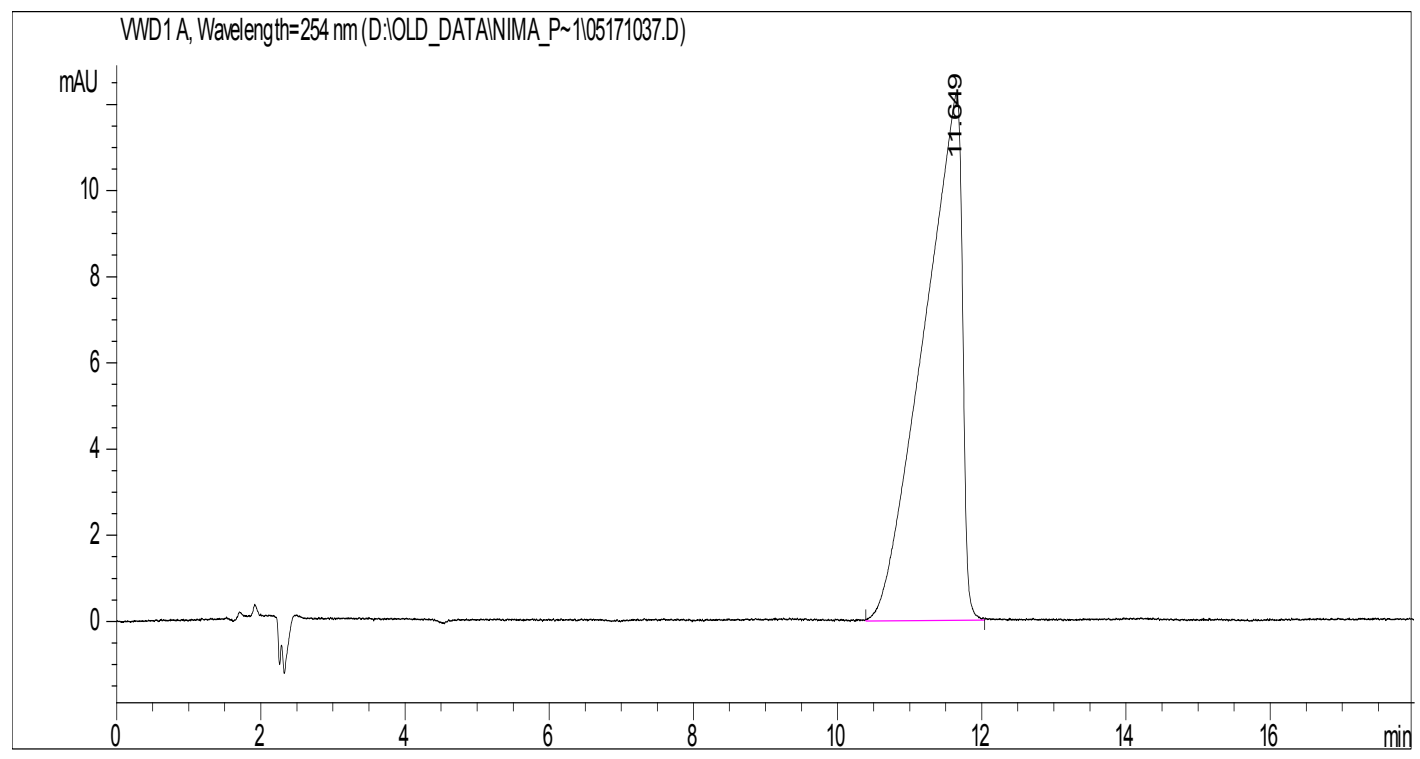

Figure 53. Chromatogram of L-tryptophan on the diamond hydride stationary phase. Mobile phase: $90: 10$ acetonitrile $+0.1 \%$ formic acid $/$ water $+0.1 \%$ formic acid 
Table 26. Retention time of L-tryptophan on the diamond hydride column using UV detection.

\begin{tabular}{|c|c|c|}
\hline \multicolumn{2}{|c|}{ Concentration (\%) } & Retention time (min) \\
\hline Acetonitrile with 0.1\% FA & DI water with 0.1\% FA & L-Tryptophan \\
\hline 50 & 50 & 2.41 \\
\hline 60 & 40 & 2.67 \\
\hline 70 & 30 & 3.23 \\
\hline 80 & 20 & 4.78 \\
\hline 90 & 10 & 11.65 \\
\hline
\end{tabular}

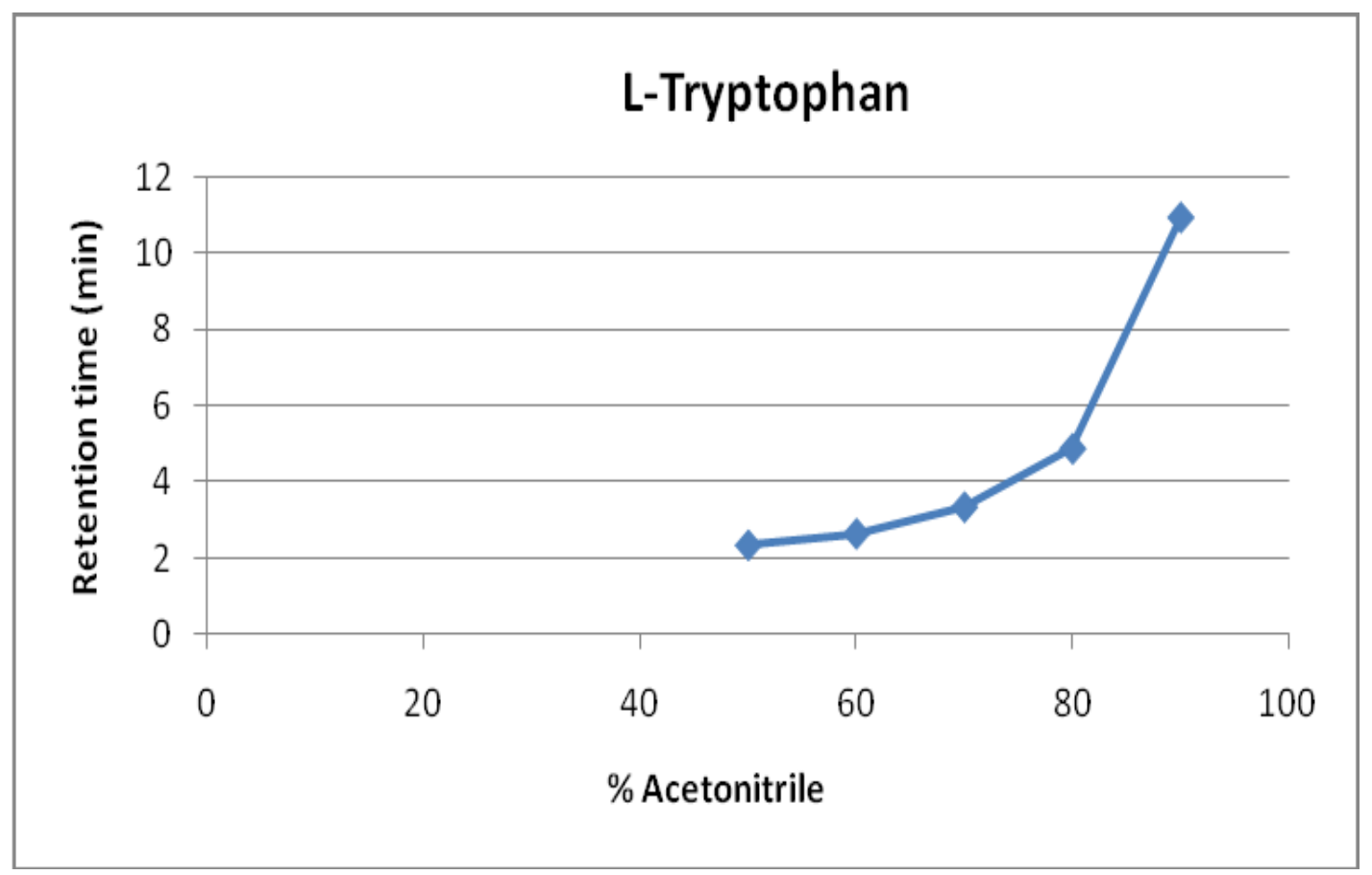

Figure 54. Retention map of L-tryptophan on the diamond hydride column using UV detection. 


\subsection{ANP Behavior of Guanidine}

Guanidine was analyzed in the ANP isocratic mode on the diamond hydride column. The concentration of guanidine used for the analysis was $0.5 \mathrm{mg} / \mathrm{mL}$. A wavelength of $254 \mathrm{~nm}$ was selected and the column was stabilized according to the starting conditions of the isocratic test.

Table 27 shows the retention time of guanidine as the concentration of acetonitrile increases from $50 \%$ to $80 \%$. Guanidine displayed very good retention for $80: 20$ acetonitrile/water (both premixed with $0.1 \%$ formic acid). Figure 55 shows the retention map of guanidine and confirms the typical ANP behavior of guanidine.

Table 27. Retention time of guanidine on the diamond hydride column using UV detection.

\begin{tabular}{|c|c|c|}
\hline \multicolumn{2}{|c|}{ Concentration (\%) } & Retention time (min) \\
\hline Acetonitrile with 0.1\% FA & DI water with 0.1\% FA & Guanidine \\
\hline 50 & 50 & 2.90 \\
\hline 60 & 40 & 3.54 \\
\hline 70 & 30 & 4.77 \\
\hline 80 & 20 & 7.72 \\
\hline
\end{tabular}




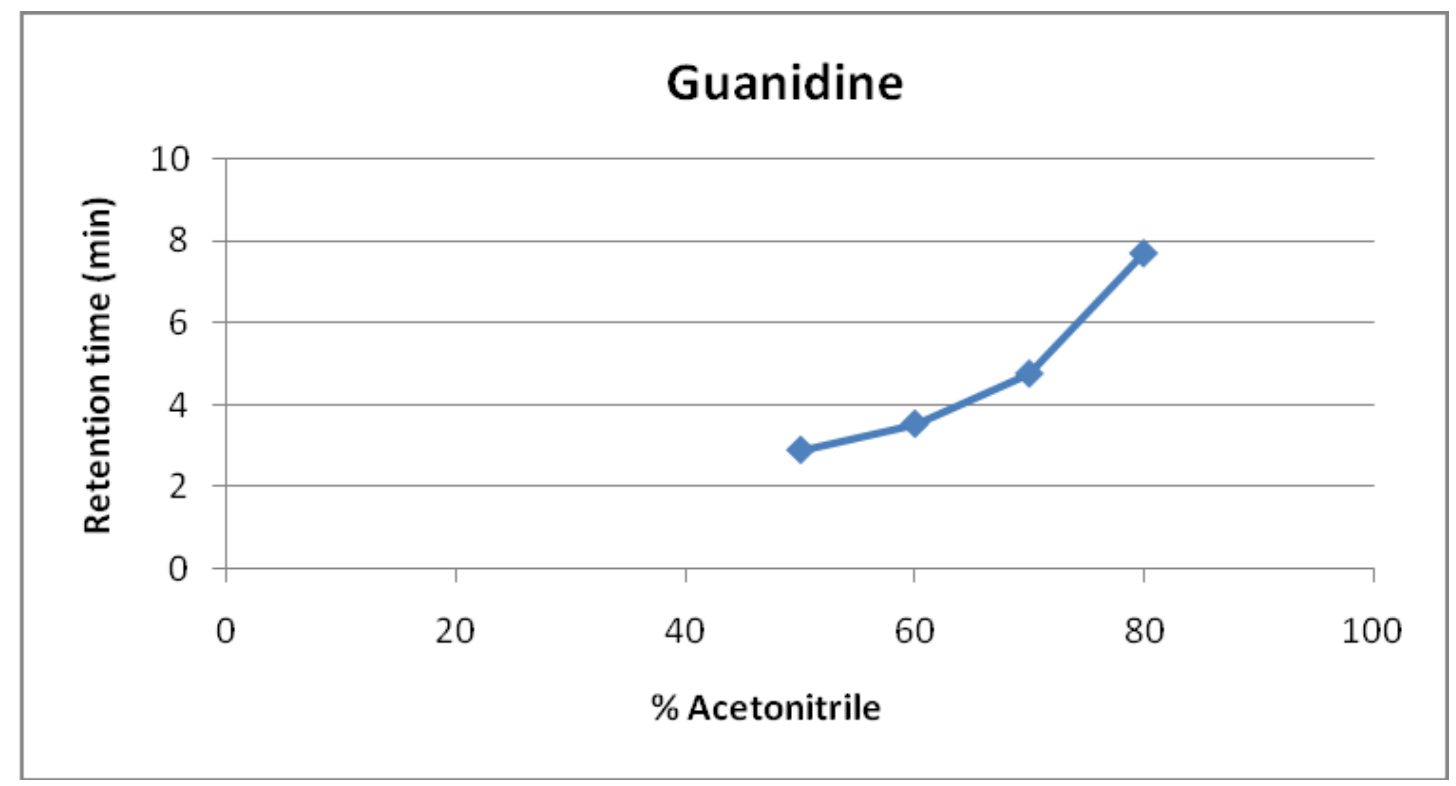

Figure 55. Retention map of guanidine on the diamond hydride column using UV detection.

\subsection{ANP Behavior of Creatinine}

Creatinine was analyzed using UV detection under ANP isocratic conditions. The compound was prepared using 50:50 acetonitrile/water (both premixed with $0.1 \%$ formic acid) at a concentration of $0.5 \mathrm{mg} / \mathrm{mL}$. A wavelength of $254 \mathrm{~nm}$ was selected and the column was stabilized according to the initial isocratic test conditions.

Table 28 shows ANP elution of creatinine as the concentration of acetonitrile increases from $50 \%$ to $80 \%$. Figure 56 represents the characteristic ANP retention map of the compound. This data indicates typical ANP behavior of creatinine on the diamond hydride column. 
Table 28. Retention time of creatinine on the diamond hydride column using UV detection.

\begin{tabular}{|c|c|c|}
\hline \multicolumn{2}{|c|}{ Concentration (\%) } & Retention time (min) \\
\hline $\begin{array}{c}\text { Acetonitrile with 0.1\% } \\
\text { FA }\end{array}$ & DI water with 0.1\% FA & Creatinine \\
\hline 50 & 50 & 3.06 \\
\hline 60 & 40 & 3.19 \\
\hline 70 & 30 & 5.81 \\
\hline 80 & 20 & 5.92 \\
\hline
\end{tabular}

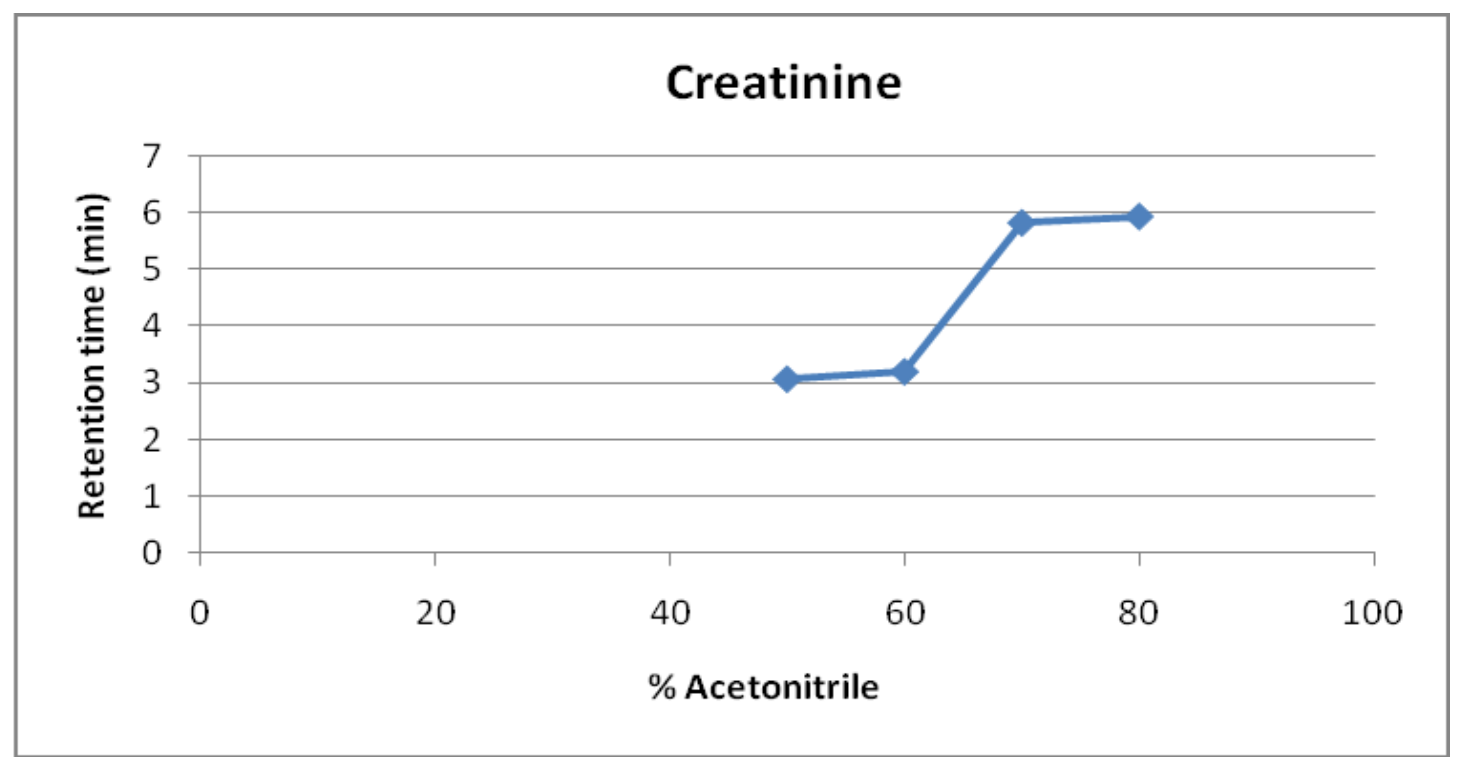

Figure 56. Retention map of creatinine on the diamond hydride column using UV detection. 


\subsection{ANP Isocratic Behavior of Primary Amines}

2,6-Diaminopurine, 2-phenethylamine and benzylamine were analyzed under ANP isocratic conditions on the diamond hydride column as shown in Figures 57 - 59 respectively. 4-Chloro-1,2-phenylenediamine, 1,2,4,5benzenetetracarboxamide and 1,2,4,5-benzenetetramine tetrahydrochloride were also analyzed under the same conditions as summarized in Table 29. For this study, all primary amines were dissolved in 50:50 acetonitrile/water (both premixed with $0.1 \%$ formic acid) at a concentration of $0.5 \mathrm{mg} / \mathrm{mL}$. A wavelength of $254 \mathrm{~nm}$ was selected and the column was stabilized according to the initial isocratic conditions. The mobile phase flow rate was set at $0.5 \mathrm{~mL} / \mathrm{min}$ with a 5 $\mu \mathrm{L}$ injection volume at ambient temperature. Table 29 and Table 30 show the retention times of the primary amines on the diamond hydride column. The retention time increases as the acetonitrile concentration increases indicating that the compound is being retained in the ANP mode at 50:50,60:40, 70:30, $80: 20,85: 15$ and $90: 10$ mobile phase compositions.

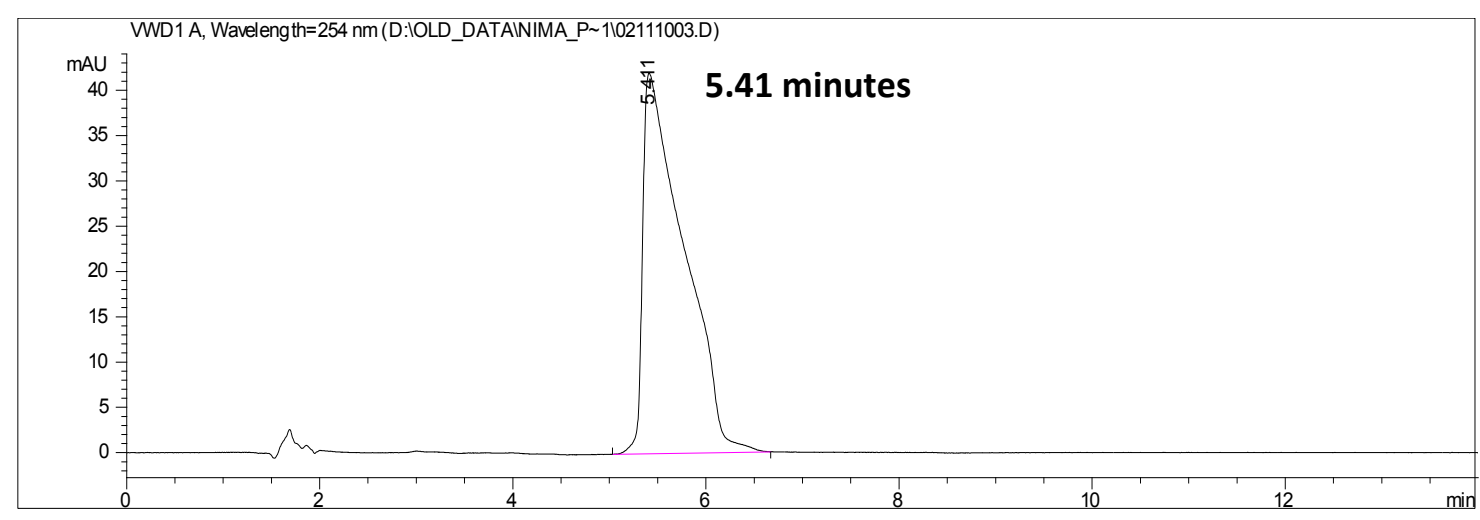

Figure 57. Chromatogram of 2-phenethylamine on the diamond hydride stationary phase. Mobile phase: $80: 20$ acetonitrile $+0.1 \%$ formic acid / water + $0.1 \%$ formic acid. 


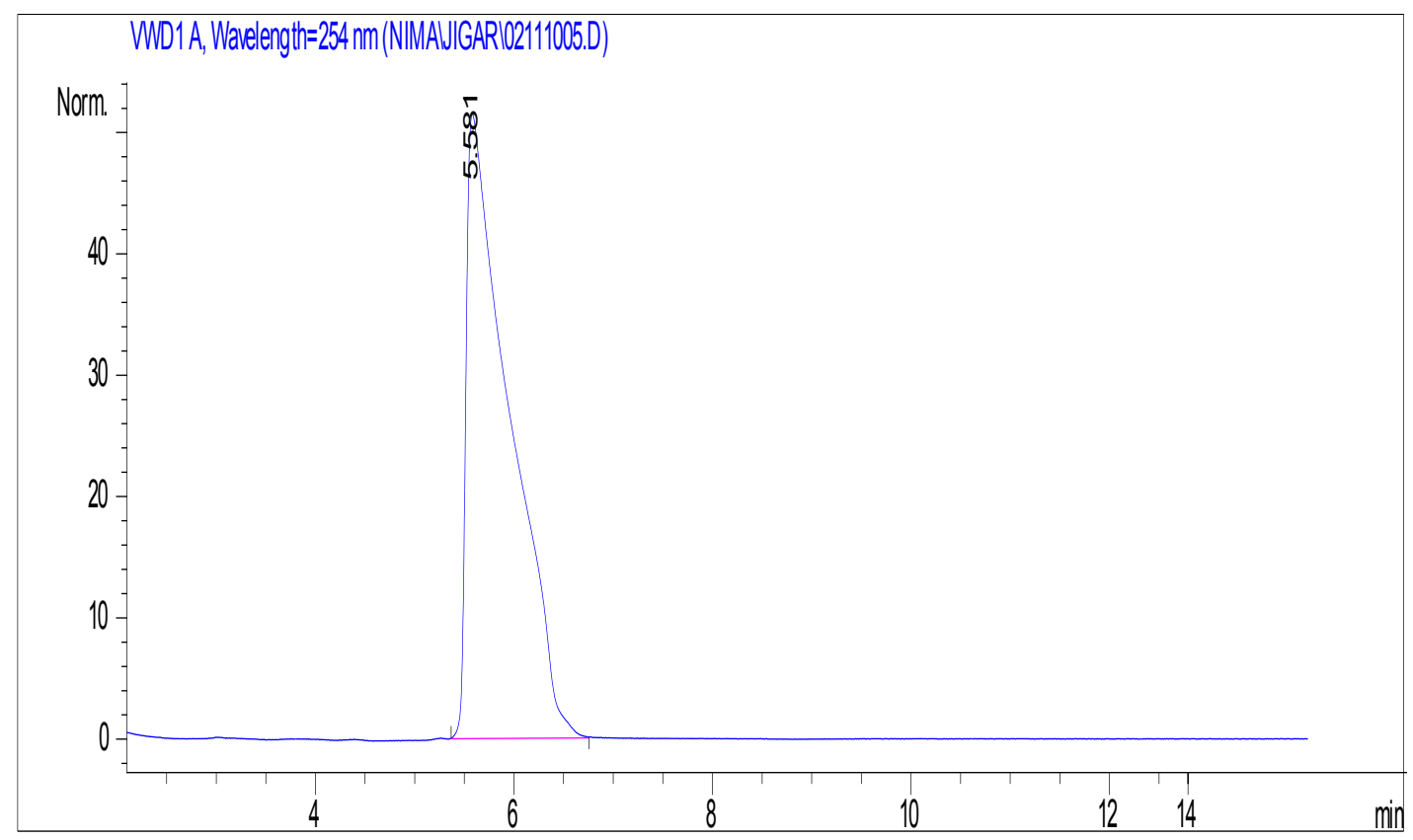

Figure 58. Chromatogram of benzylamine on the diamond hydride stationary phase. Mobile phase: $80: 20$ acetonitrile $+0.1 \%$ formic acid / water $+0.1 \%$ formic acid.

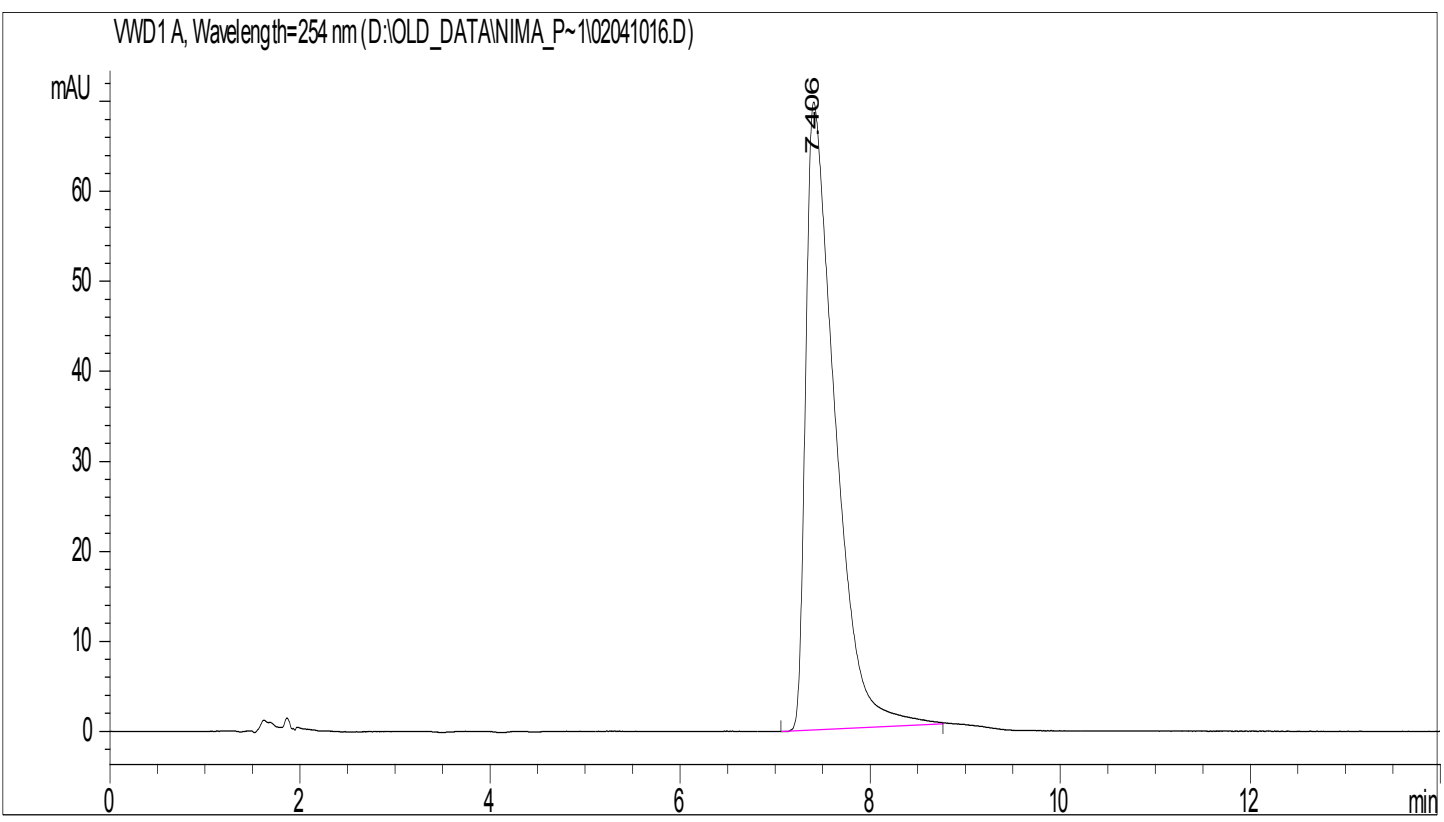

Figure 59. Chromatogram of 2,6-diaminopurine on the diamond hydride stationary phase. Mobile phase: $80: 20$ acetonitrile $+0.1 \%$ formic acid / water + $0.1 \%$ formic acid. 
Table 29. Summary of the retention times of primary amines on the diamond hydride column using UV.

\begin{tabular}{|c|c|c|c|c|}
\hline \multicolumn{2}{|c|}{ Concentration (\%) } & \multicolumn{3}{|c|}{ Retention time(min) } \\
\hline Acetonitrile & Water & $\begin{array}{c}\mathbf{1 , 2 , 4 , 5 -} \\
\text { Benzenetetramine } \\
\text { te.1\% FA) }\end{array}$ & $\begin{array}{c}\text { 4-Chloro-1,2- } \\
\text { phenethylene- } \\
\text { diamine }\end{array}$ & $\begin{array}{c}\mathbf{1 , 2 , 4 , 5 -} \\
\text { Benzenetetra- } \\
\text { carboxamide }\end{array}$ \\
\hline 50 & 50 & 3.08 & 2.97 & 1.72 \\
\hline 60 & 40 & 4.76 & 3.13 & 1.80 \\
\hline 70 & 30 & 4.77 & 3.34 & 1.97 \\
\hline 80 & 20 & 6.70 & 3.82 & 2.33 \\
\hline 85 & 15 & 9.89 & 4.30 & 2.46 \\
\hline 90 & 10 & 12.89 & 5.04 & 2.83 \\
\hline
\end{tabular}

Table 30. Summary of the retention times of primary amines on the diamond hydride column using UV.

\begin{tabular}{|c|c|c|c|c|}
\hline \multicolumn{2}{|c|}{ Concentration (\%) } & \multicolumn{3}{|c|}{ Retention time(min.) } \\
\hline $\begin{array}{c}\text { Acetonitrile } \\
(0.1 \% \text { FA) }\end{array}$ & \begin{tabular}{c|} 
Water \\
\\
$(0.1 \%$ \\
FA $)$ \\
\end{tabular} & Benzylamine & $\begin{array}{l}\text { 2-Phenethyl- } \\
\text { amine }\end{array}$ & 2,6-Diaminopurine \\
\hline 50 & 50 & 2.75 & 2.77 & 3.37 \\
\hline 60 & 40 & 2.85 & 2.87 & 4.04 \\
\hline 70 & 30 & 3.57 & 3.55 & 5.16 \\
\hline 80 & 20 & 5.58 & 5.41 & 7.40 \\
\hline 85 & 15 & 14.07 & 13.82 & 11.8 \\
\hline 90 & 10 & - & - & - \\
\hline
\end{tabular}


In addition to the compounds above, one more primary amine, 3,4diaminobenzophenone was analyzed using the same ANP conditions and mobile phase. As shown in Table 31, 3,4-diaminobenzophenone was not retained on the diamond hydride column eluting at the void volume for increasing acetonitrile concentrations. The insignificant ANP retention of this primary amine is likely due to the stated experimental condition. The conclusion was drawn that this primary amine cannot be retained on the diamond hydride column using the experimental conditions tested. Therefore, more work needs to be done in order to determine if it is possible to retain this compound on the diamond hydride column in the ANP mode.

Table 31. Summary of isocratic elution data for 3,4-diaminobenzophenone.

\begin{tabular}{|c|c|c|}
\hline \multicolumn{2}{|c|}{ Concentration (\%) } & Retention time (min) \\
\hline $\begin{array}{c}\text { Acetonitrile with 0.1\% } \\
\text { FA }\end{array}$ & DI water with 0.1\% FA & 3,4-Diaminobenzophenone \\
\hline 50 & 50 & 2.13 \\
\hline 60 & 40 & 2.14 \\
\hline 70 & 30 & 2.07 \\
\hline 80 & 20 & 2.01 \\
\hline 90 & 10 & 2.04 \\
\hline
\end{tabular}




\subsection{ANP Behavior of Diamond Hydride Column with Gradient Elution Using UV Detection}

Primary amines were analyzed in the ANP gradient mode. Table 8 and Table 9 list the gradient programs used for this work. A wavelength of $254 \mathrm{~nm}$ was selected and the column was stabilized according to the starting conditions of the gradient. The flow rate was set at $0.5 \mathrm{~mL} / \mathrm{min}$ with a $5 \mu \mathrm{L}$ injection volume at ambient temperature. The concentration used was $0.5 \mathrm{mg} / \mathrm{mL}$ in acetonitrile/water (both premixed with $0.1 \%$ formic acid). A water blank was run to eliminate any contamination from the previous measurements. The main goal of this part of the research was to evaluate the diamond hydride stationary phase for aqueous normal phase behavior under gradient conditions.

2,6-Diaminopurine was analyzed using Gradient B, shown in Table 8. It can be seen from the chromatogram in Figure 60 that the compound was eluted at 6.47 min using Gradient B. The mobile phase used for this analysis was acetonitrile ( $0.1 \%$ formic acid) and water ( $0.1 \%$ formic acid). Figure 60 shows the enhancement of the retention capabilities of the diamond hydride column for a polar compound using aqueous normal phase conditions. 


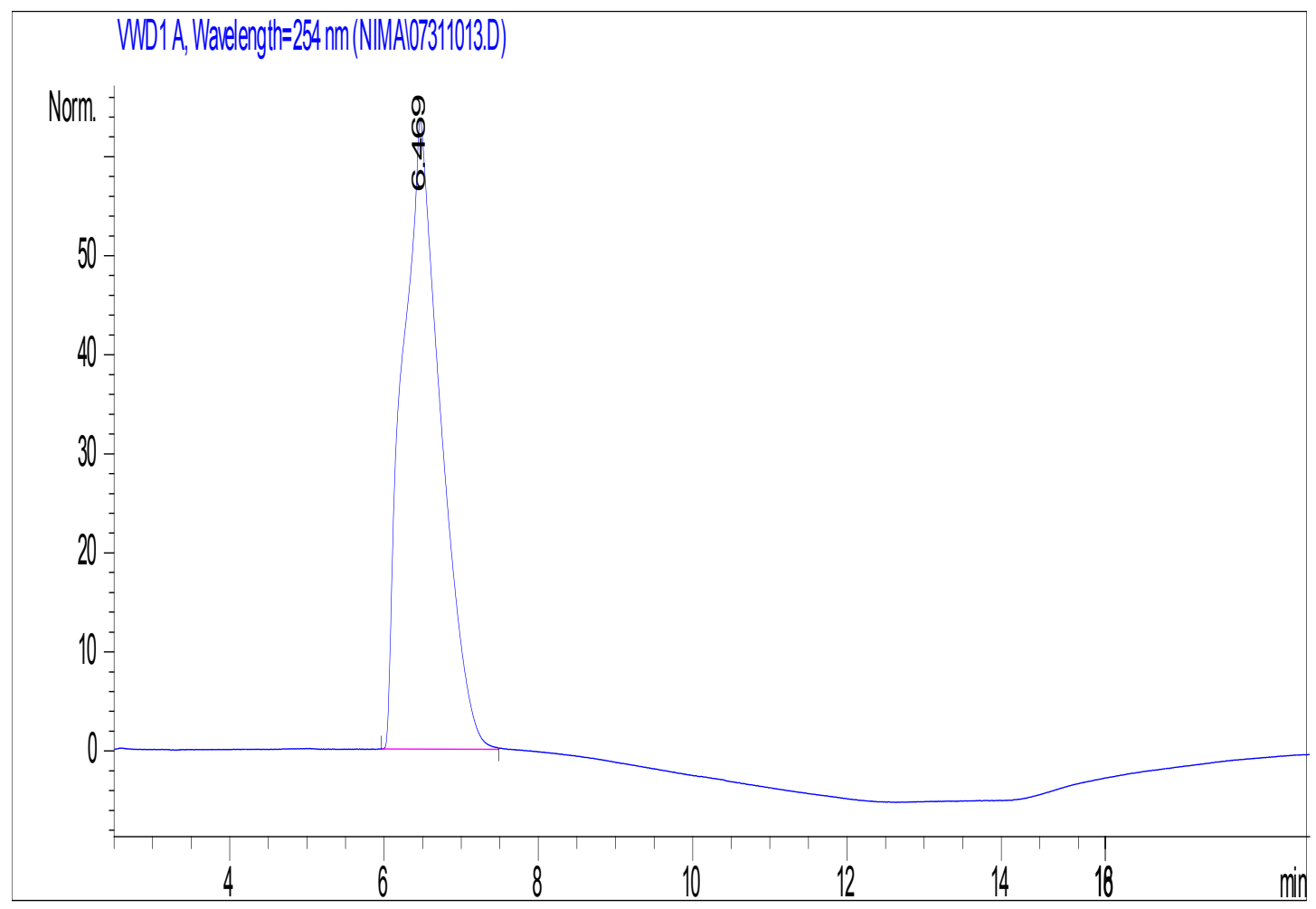

Figure 60. Chromatogram of 2,6-diaminopurine on the diamond hydride column. Mobile phase Gradient B.

Using ANP gradient conditions, 1,2,4,5-benzenetetramine tetrahydrochloride was analyzed on the diamond hydride column shown in Figure 61. The compound eluted at 3.39 minutes using Gradient A. The mobile phase used was acetonitrile/water (both premixed with $0.1 \%$ formic acid). $1,2,4,5-$ Benzenetetramine tetrahydrochloride showed a highly efficient symmetric peak on the diamond hydride column using Gradient A. The retention times of all the primary amines analyzed on the diamond hydride column using different gradient modes are presented in Table 32. 


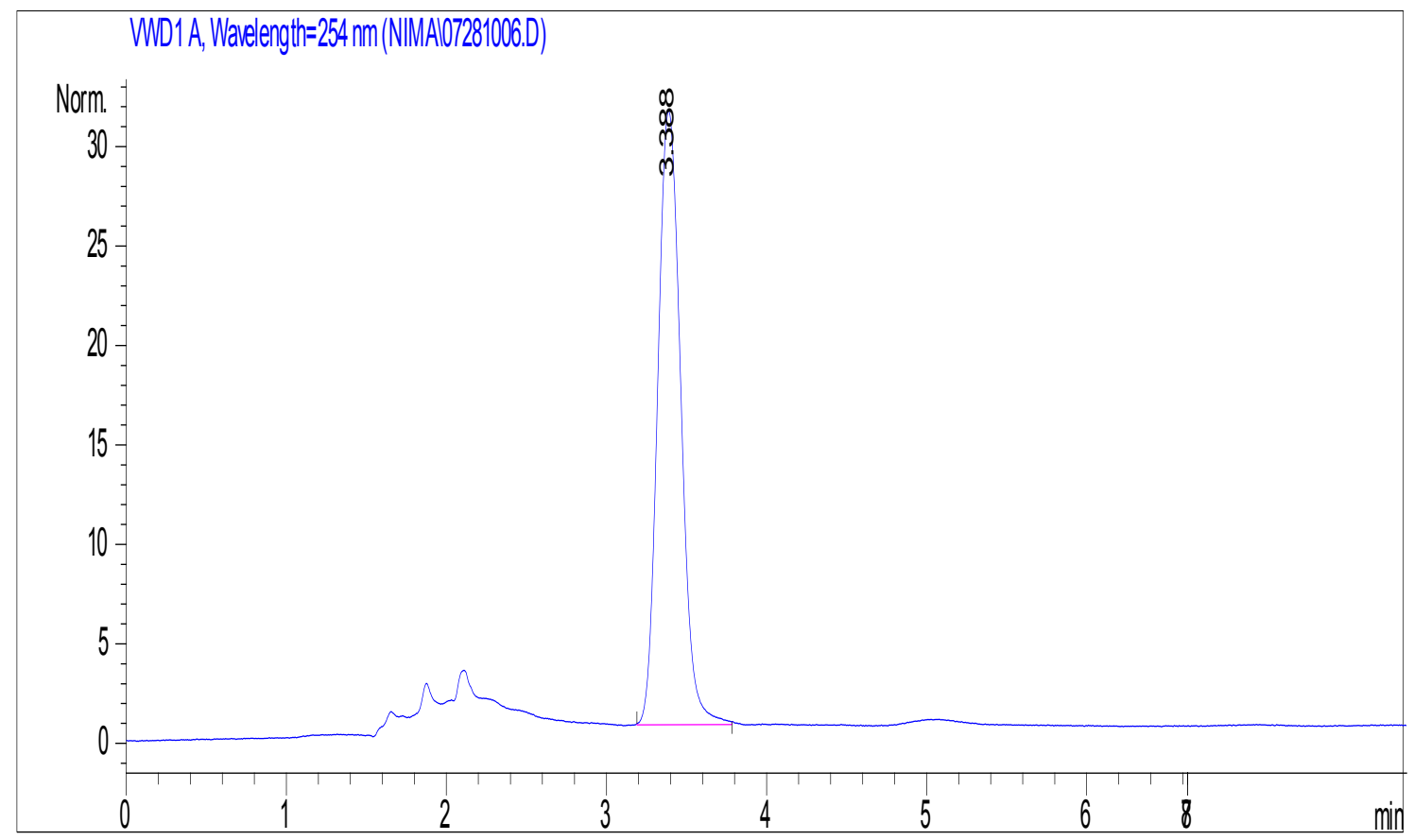

Figure 61. Chromatogram of 1,2,4,5-benzenetetramine tetrahydrochloride on the diamond hydride column. Mobile phase Gradient A.

Table 32. ANP gradient retention of primary amines on the diamond hydride column.

\begin{tabular}{|c|c|c|c|}
\hline \multirow{2}{*}{ Primary amine } & \multicolumn{3}{|c|}{ Retention time } \\
\cline { 2 - 4 } & Gradient A & Gradient B & Gradient D \\
\hline 2,6-Diaminopurine & 11.11 & 6.47 & 12.45 \\
\hline 2-Phenethylamine & 9.15 & 4.19 & 11.02 \\
\hline Benzylamine & & & \\
\hline 4-Chloro-1,2-phenylenediamine & 3.01 & 4.08 & 11.13 \\
& & & \\
\hline 1,2,4,5-Benzenetetramine tetrahydrochloride & 3.39 & 2.78 & 3.19 \\
& & & \\
\hline
\end{tabular}




\subsection{ANP Separation of Two Compounds Using Gradient Method}

All the compounds used for the gradient elution were already analyzed using isocratic conditions so their behavior and retention were known on the diamond hydride column. Different gradient conditions were created to separate mixtures of primary amines. The retention time varied from gradient to gradient depending on the increment of the percent organic component in the mobile phase composition. The retention time for each of the compounds was confirmed by running the single analytes using the same mobile phase, gradient method, instrument and all other parameters. The main goal of this work was to study the behavior of diamond hydride stationary phase for aqueous normal phase behavior.

Figure 62 shows the separation of a mixture 1,2,4,5-benzenetetramine tetrahydrochloride and 2,6-diaminopurine using Gradient A listed in Table 8 in the ANP gradient mode. Both compounds were dissolved in $50: 50$ acetonitrile $(0.1 \%$ formic acid)/water ( $0.1 \%$ formic acid) at a concentration of $0.5 \mathrm{mg} / \mathrm{mL}$. A wavelength of $254 \mathrm{~nm}$ was used and the column was stabilized according to the initial conditions of the gradient. The injection volume used for all of the separations was $5 \mu \mathrm{L}$ with a flow rate at $0.5 \mathrm{~mL} / \mathrm{min}$. 2,6-Diaminopurine being more polar compared to 1,2,4,5-benzenetetramine tetrahydrochloride eluted at 11.05 minutes and 1,2,4,5-benzenetetramine tetrahydrochloride eluted at 3.42 minutes. Both compounds showed good retention and are well separated on the diamond hydride column. 
The chromatographic separation of 1,2,4,5-benzenetetramine tetrahydrochloride and 2,6-diaminopurine using Gradient B is shown in Figure 63. It can be seen from the Figure 63 that the diamond hydride column has successfully separated both compounds. 1,2,4,5-Benzenetetramine tetrahydrochloride is less polar compared to 2,6-diaminopurine and eluted first at 2.60 minutes, very close to the void volume, and 2,6-diaminopurine eluted at 6.60 minutes.

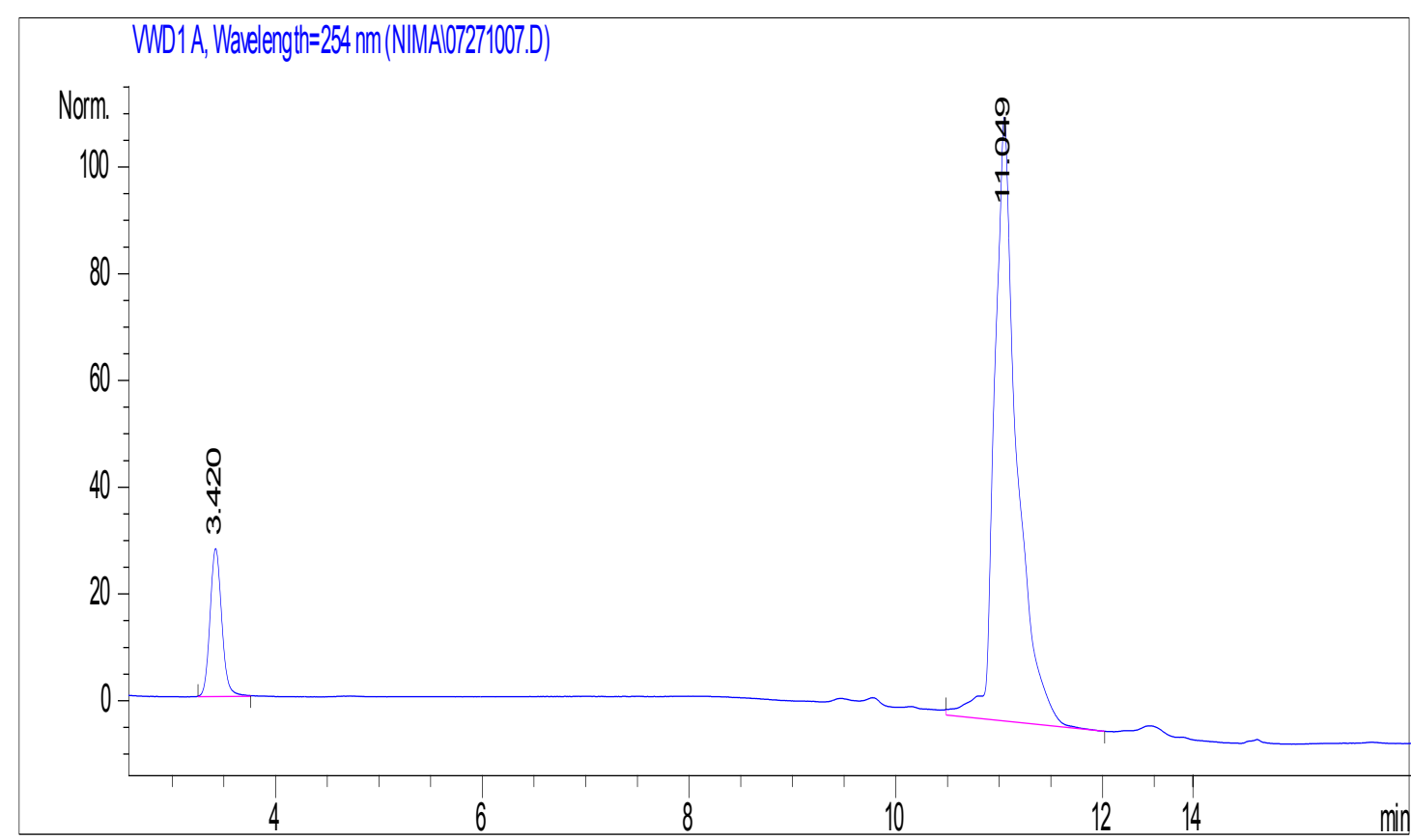

Figure 62. Aqueous normal phase gradient separation of 1,2,4,5benzenetetramine tetrahydrochloride and 2,6-diaminopurine on the diamond hydride column. The peak at 3.42 minutes is for 1,2,4,5-benzenetetramine tetrahydrochloride and the peak at 11.05 minutes is for 2,6-diaminopurine. Mobile phase Gradient A. 


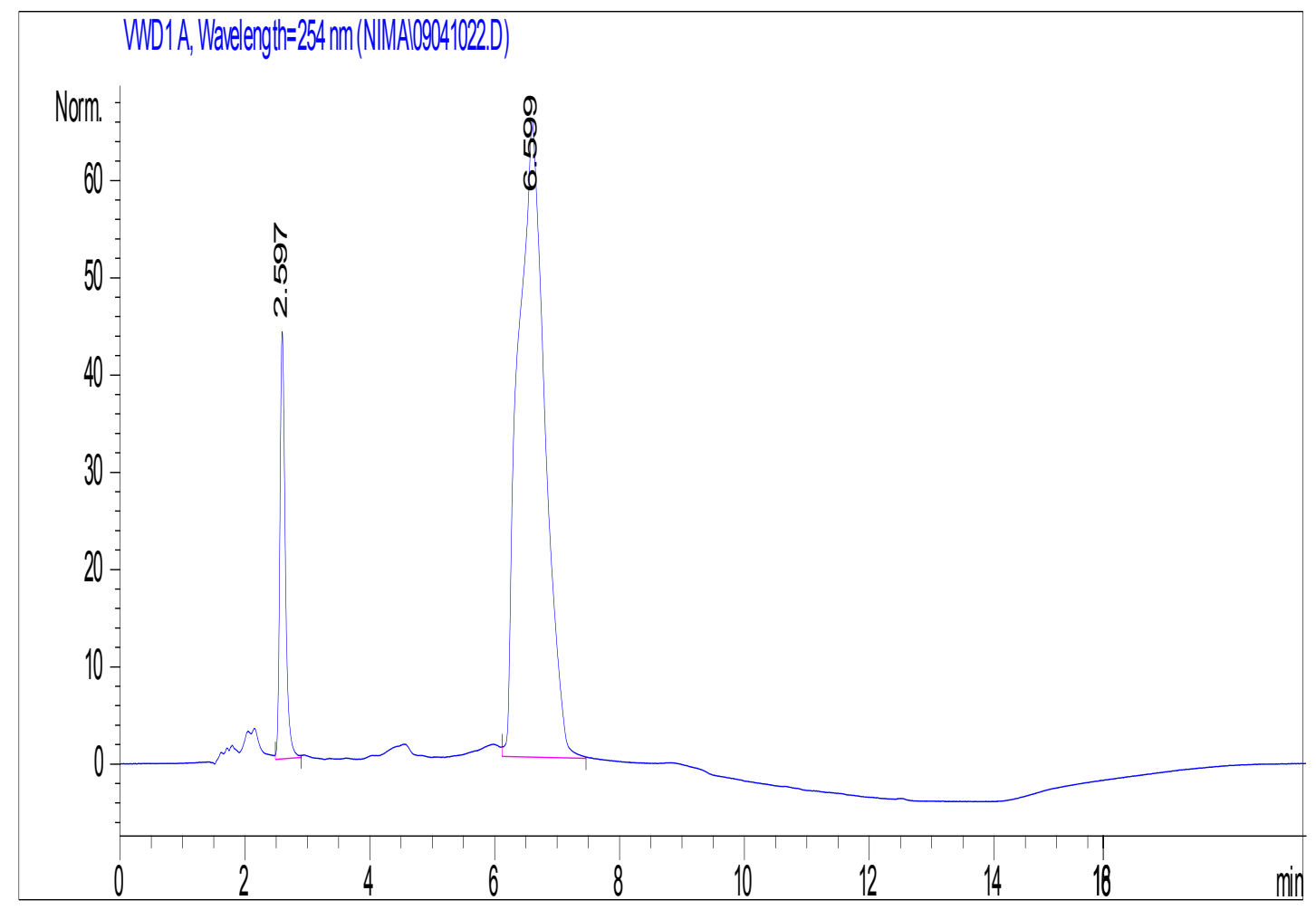

Figure 63. Aqueous normal phase gradient separation of 1,2,4,5benzenetetramine tetrahydrochloride and 2,6-diaminopurine on the diamond hydride column. The peak at 2.60 minutes is for 1,2,4,5-benzenetetramine tetrahydrochloride and the peak at 6.60 minutes is for 2,6-diaminopurine.Mobile phase Gradient B.

The mixture of two compounds, 2-phenethylamine and 2,6diaminopurine was separated using Gradient A as shown Figure 64. It can be seen from Figure 64 that the diamond hydride column has successfully separated both compounds and 2,6 diaminopurine was retained longer on the column because it is more polar compared to 2-phenethylamine. 2,6Diaminopurine eluted at 11.25 minutes and 2-phenethylamine eluted at $9.94 \mathrm{~min}$. 


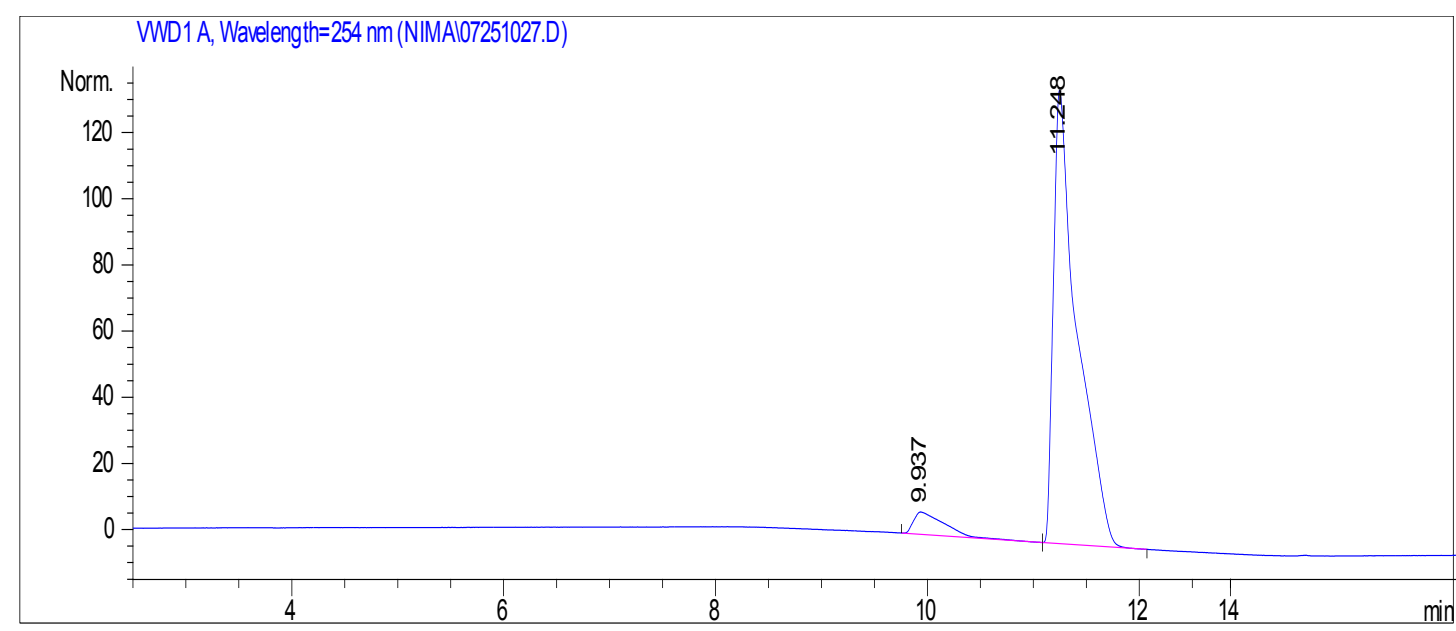

Figure 64. Aqueous normal phase gradient separation of 2-phenethylamine and 2,6-diaminopurine on the diamond hydride column. The peak at 9.94 minutes is for 2-phenethylamine and the peak at 11.25 minutes is for 2,6- diaminopurine. Mobile phase Gradient A.

\section{2. $\quad$ ANP Separation of Three Compounds Using Gradient Method}

A mixture of three primary amines was analyzed on the diamond hydride column under aqueous normal phase gradient conditions. After confirming the retention of all primary amines on the diamond hydride column using different gradient programs, a mixture of three compounds was tested. The gradient programs used for this study are shown in Table 8 and Table 9. The flow rate was set at $0.5 \mathrm{~mL} / \mathrm{min}$ and a $5 \mu \mathrm{L}$ injection volume was used. The detector used for this analysis was UV at a wavelength of $254 \mathrm{~nm}$. All compounds were dissolved in $50: 50$ acetonitrile $(0.1 \%$ formic acid $) /$ water $(0.1 \%$ formic acid $)$ at a concentration of $0.5 \mathrm{mg} / \mathrm{mL}$. All the separations were performed at ambient temperature. The main goal of this work was to analyze the behavior of primary amines on the diamond hydride column and the following discussion will provide some details regarding this part of the investigation. 
As shown in Figure 65, 1,2,4,5-benzenetetramine tetrahydrochloride, benzylamine and 2,6-diaminopurine were retained on the diamond hydride column at $3.43,9.53$ and 10.78 minutes respectively. The analysis was carried out using Gradient A shown in Table 8. It can be seen from the chromatogram in Figure 65 that 1,2,4,5-benzenetetramine tetrahydrochloride eluted first at 3.43 minutes since it is the least polar compound in the mixture. Benzylamine eluted at 9.53 minutes and 2,6-diaminopurine eluted at 10.78 minutes.

From Figure 65 it can be observed that the peak shapes of 1,2,4,5benzenetetramine tetrahydrochloride and 2,6-diaminopurine were sharp and symmetric whereas benzylamine showed peak tailing. This can be solved by optimizing gradient conditions. Therefore more work needs to be done in order to improve the appearance of the chromatogram.

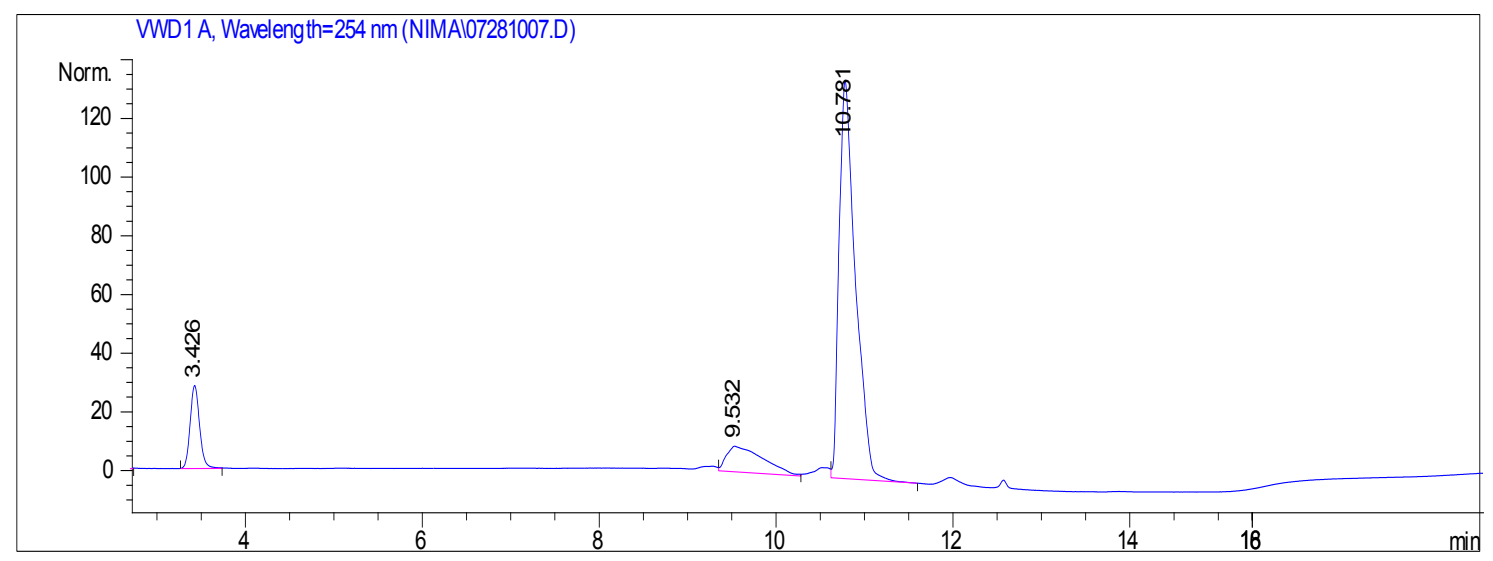

Figure 65. Aqueous normal phase gradient separation of 1,2,4,5benzenetetramine tetrahydrochloride, benzylamine and 2,6-diaminopurine on the diamond hydride column. The peak at 3.43 minutes is for $1,2,4,5-$ benzenetetramine tetrahydrochloride, peak at 9.53 minutes is for benzylamine and the peak at 10.78 minutes is for 2,6-diaminopurine. Mobile phase Gradient A. 
Another mixture of three primary amines was tested on the diamond hydride column under aqueous normal phase gradient conditions. Figure 66 shows the separation of 1,2,4,5-benzenetetramine tetrahydrochloride, 2phenethylamine and 2,6-dimainopurine. The analysis was carried out using Gradient A at ambient temperature. As can be seen, the diamond hydride column successfully separated all three compounds. The peak shapes of these compounds were sharp and symmetric. 1,2,4,5-Benzenetetramine tetrahydrochloride being the least polar compound in the mixture, eluted first at 4.51 minutes whereas 2-phenethylamine eluted at 11.27 minutes. 2,6-

Diaminopurine had the longest retention on the column and eluted at 12.40 minutes. In general, the diamond hydride column has demonstrated the ability to separate mixtures of primary amines using different gradient programs.

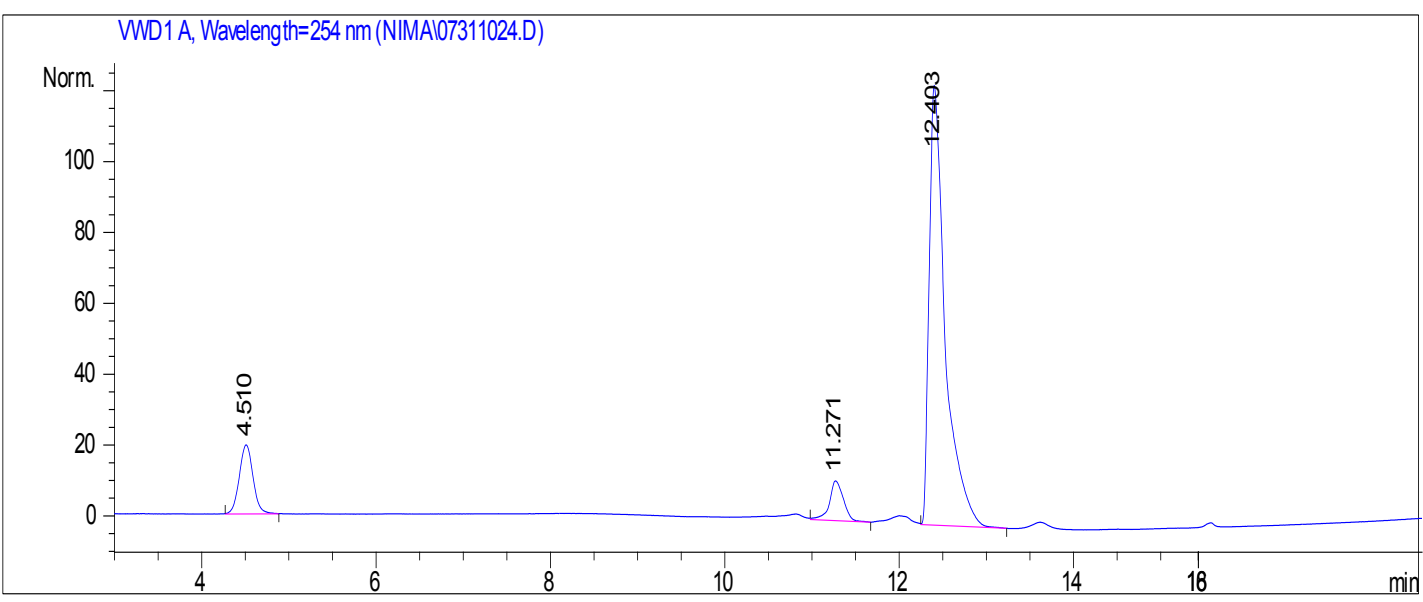

Figure 66. Aqueous normal phase gradient separation of 1,2,4,5benzenetetramine tetrahydrochloride, 2-phenethylamine and 2,6-diaminopurine on the diamond hydride column. The peak at 4.51 minutes is for 1,2,4,5benzenetetramine tetrahydrochloride, peak at 11.27 minutes is for 2 phenethylamine and the peak at 12.4 minutes is for 2,6-daminopurine. Mobile phase Gradient D. 


\section{3. $\quad$ ANP Separation of Four Compounds Using Gradient Method}

Figure 67 shows the separation of 4-chloro-1,2-phenylenediamine, benzylamine, 2,6-diaminopurine and 1,2,4,5-benzenetetramine tetrahydrochloride. After confirming their individual retention on the column using gradient conditions, a mixture of the four compounds was tested. This analysis was carried out using ANP gradient conditions on the diamond hydride column with the UV detector. The separation was undertaken using the Gradient A program shown in Table 8. 4-Chloro-1,2-phenylenediamine and 1,2,4,5benzenetetramine tetrahydrochloride eluted first at 2.94 minutes as a single peak showing the lowest retention on the column. 4-Chloro-1,2-phenylenediamine and 1,2,4,5-benzenetetramine tetrahydrochloride showed a marginal retention time difference of 3.46 minutes and 3.39 minutes respectively when tested as a single compound using Gradient A. The chromatogram showed one extra peak before the main peak of 4-chloro-1,2-phenylenediamine and 1,2,4,5-benzenetetramine tetrahydrochloride. In order to identify this extra peak, a water blank was run which showed a flat baseline without any peak. Therefore, this extra peak suggested that an impurity was introduced during the preparation of the sample solution. Benzylamine eluted at 9.01 minutes followed by 2,6-diaminopurine. 2,6Diaminopurine was the last compound to elute from the column at 10.56 minutes which had the highest retention for this compound on the diamond hydride column. 


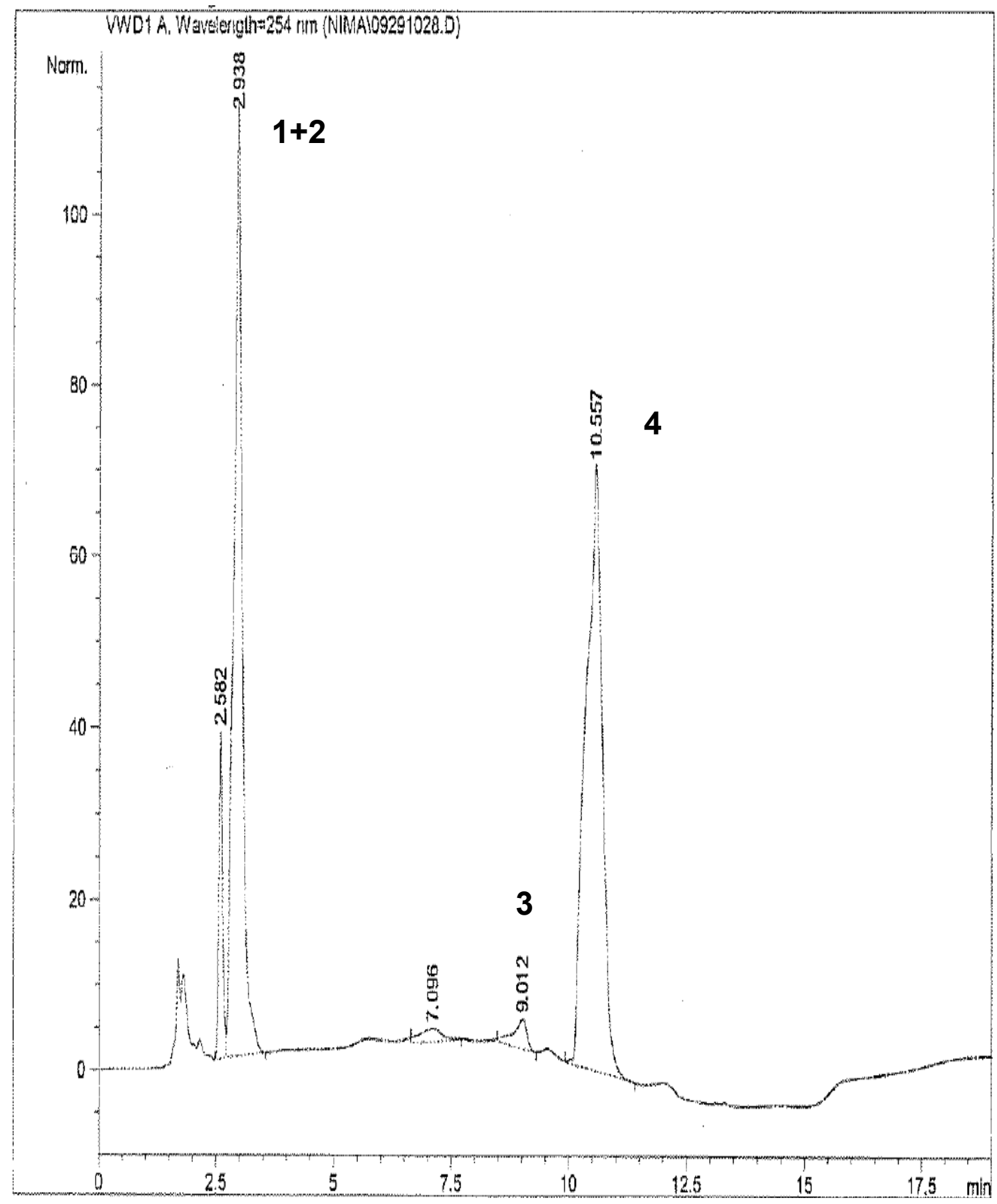

Figure 67. ANP gradient separation of 4-chloro-1,2-phenylenediamine, 1,2,4,5benzenetetramine tetrahydrochloride, benzylamine and 2,6-diaminopurine on the diamond hydride column. Peak identification: 1+2 = 4-chloro-1,2phenylenediamine and 1,2,4,5-benzenetetramine tetrahydrochloride, peak $3=$ benzylamine, peak $4=2,6$-daminopurine. Mobile phase Gradient A. 


\subsection{Comparison of the Silica Hydride and Diamond Hydride Columns Under ANP Conditions}

Figure 68 compares the retention of adenosine on the silica hydride and diamond hydride columns. As can be seen, both columns show a very similar retention profile for adenosine but the compound retained slightly longer on the diamond hydride column at 90:10 acetonitrile/water (both premixed with 0.1\% formic acid) compared to the silica hydride column. A small percentage of carbon on the diamond hydride column adjusts the hydrophobicity to a somewhat different level than that of the silica hydride column.

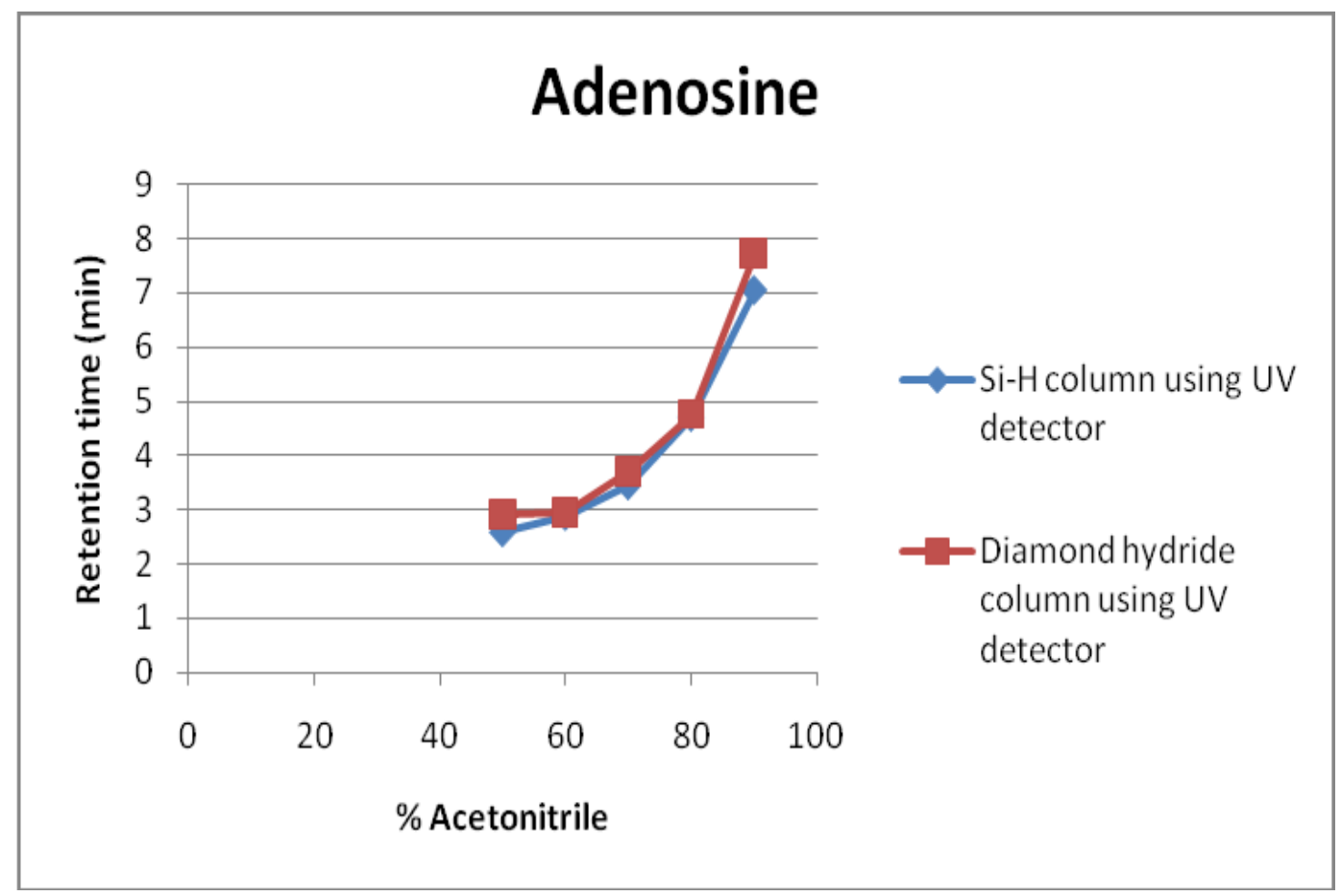

Figure 68. Comparison of retention maps of adenosine on the silica hydride and diamond hydride columns using UV detection. 
Figure 69 demonstrates the characteristic retention maps of creatinine on the silica hydride and diamond hydride columns. Cyclic creatinine shows comparatively higher retention on the silica hydride column which has a slightly more polar surface for retention than that of the diamond hydride column. Hence, it can be postulated that some polar compounds like creatinine retain longer on the silica hydride column due to the polarity of the surface.

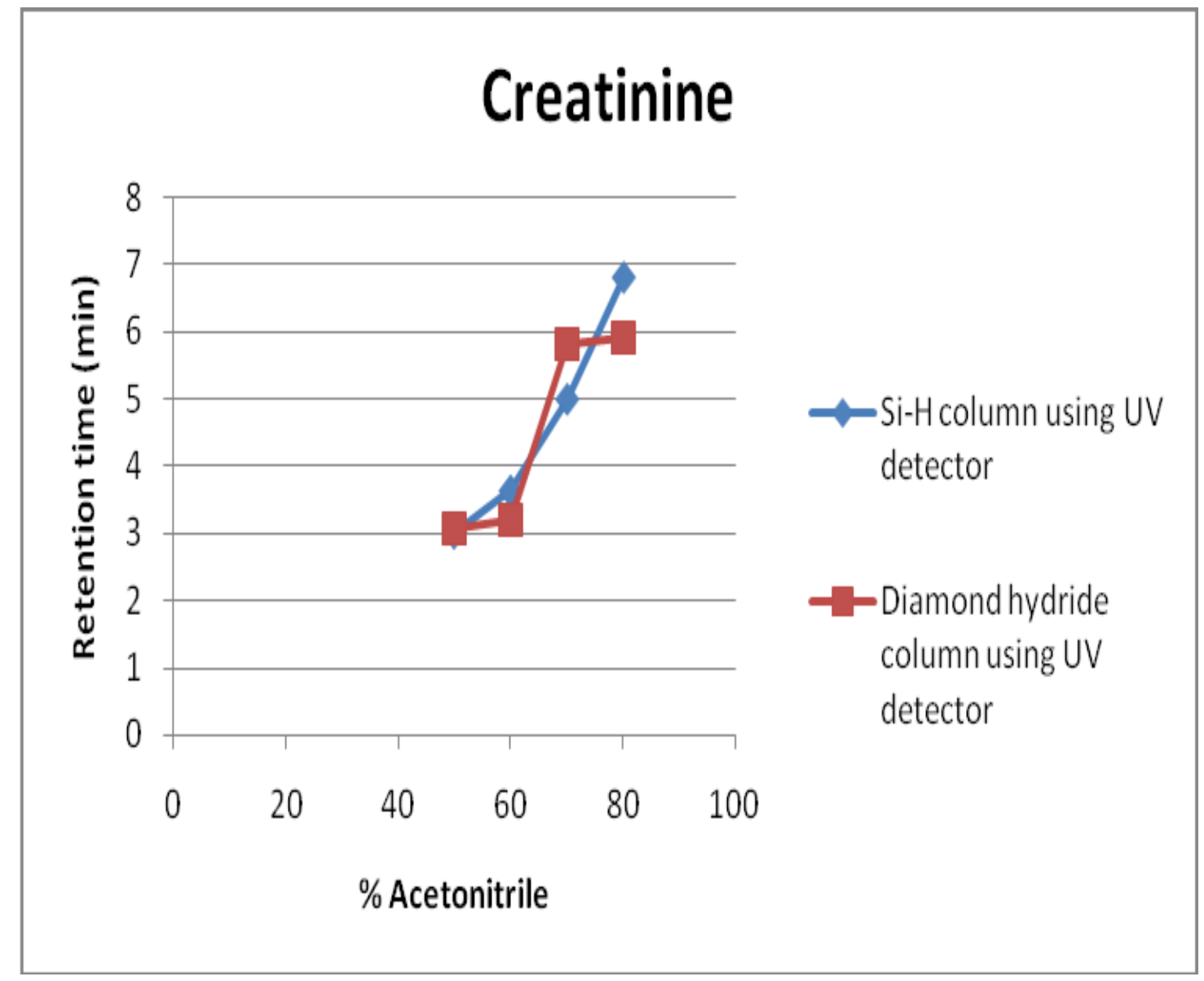

Figure 69. Comparison of retention maps of creatinine on the silica hydride and diamond hydride columns using UV detection. 
Figure 70 compares the retention of guanidine on the silica hydride and diamond hydride columns. As can be seen, guanidine shows higher ANP retention on the silica hydride column compared to the diamond hydride column. Hence, this observation also supports the fact that a higher polarity of the silica hydride surface results in longer retention for certain hydrophilic compounds.

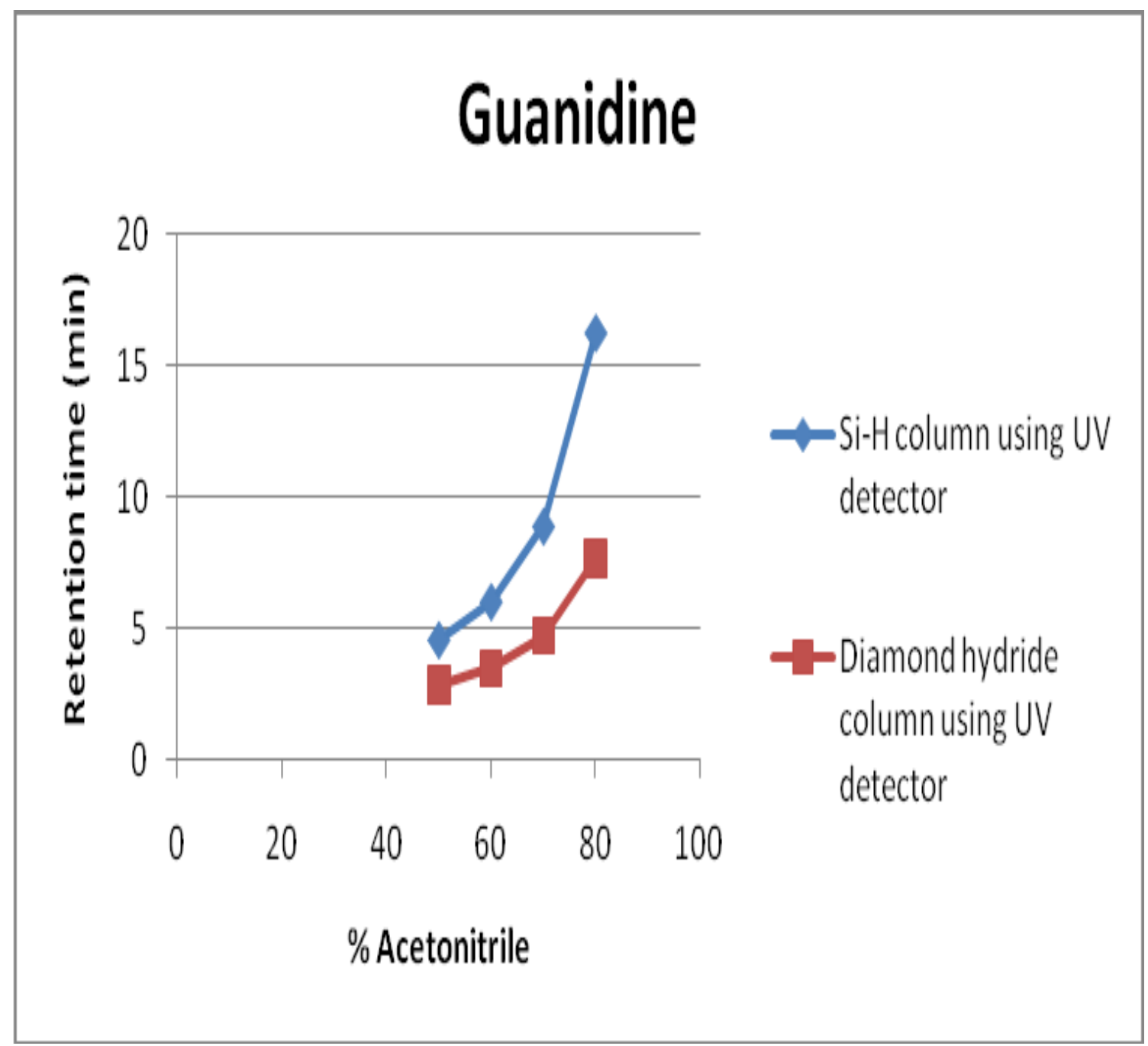

Figure 70. Comparison of retention maps of guanidine on the silica hydride and diamond hydride columns using UV detection. 
Figure 71 shows the characteristic retention maps of L-tryptophan on both columns as the amount of acetonitrile increases. As can be seen, Ltryptophan having a significant non polar component retains longer on the diamond hydride column which has a small percentage of carbon on the surface that is responsible for the hydrophobic retention.

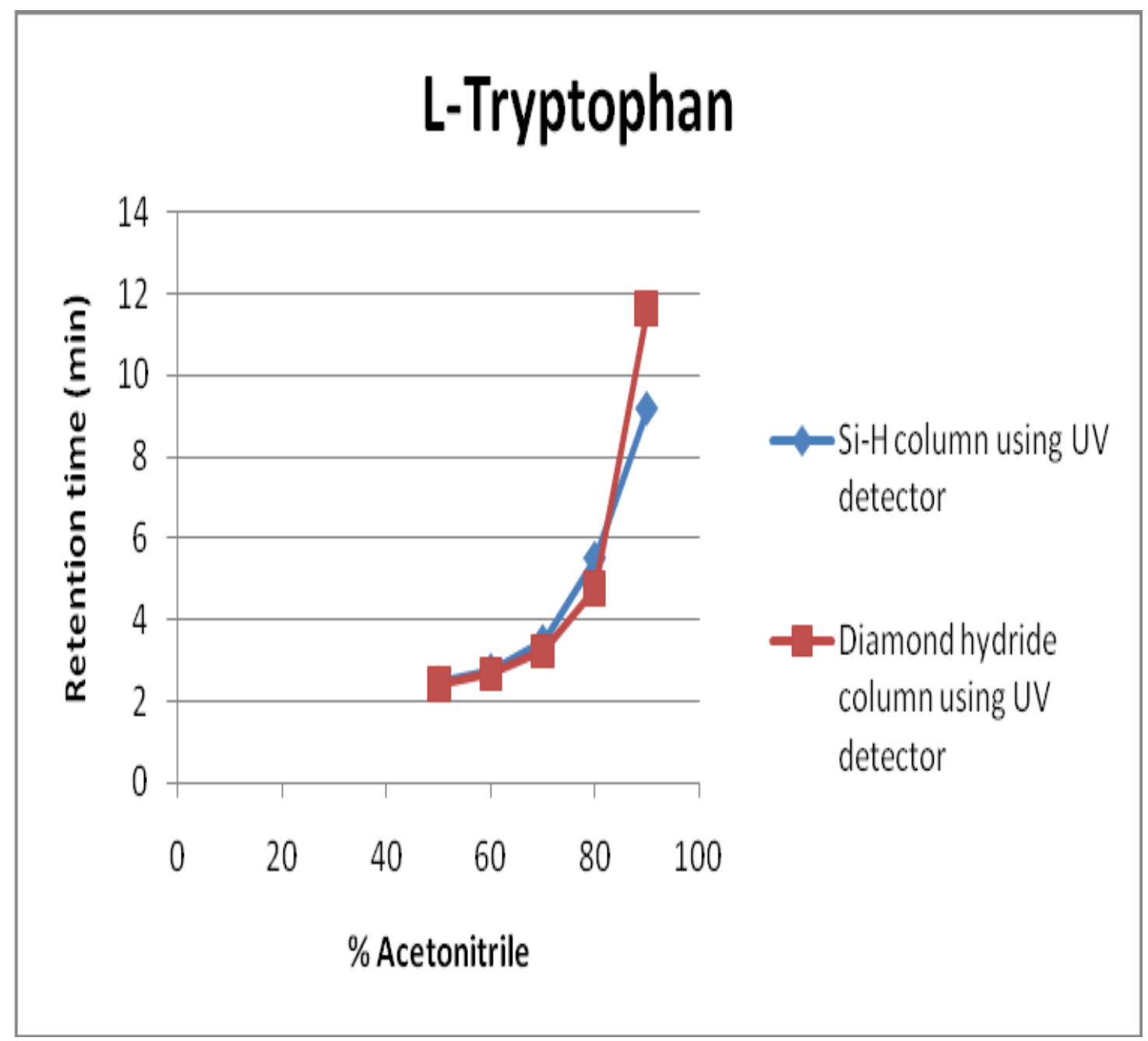

Figure 71. Comparison of retention maps of L-tryptophan on the silica hydride and diamond hydride columns using UV detection. 
Figure 72 compares the retention of guanine on the silica hydride and diamond hydride columns. The hydrophilic nucleobase guanine shows comparatively higher retention on the silica hydride column which has a more polar surface for retention than that of the diamond hydride column which has a slightly less polar surface.

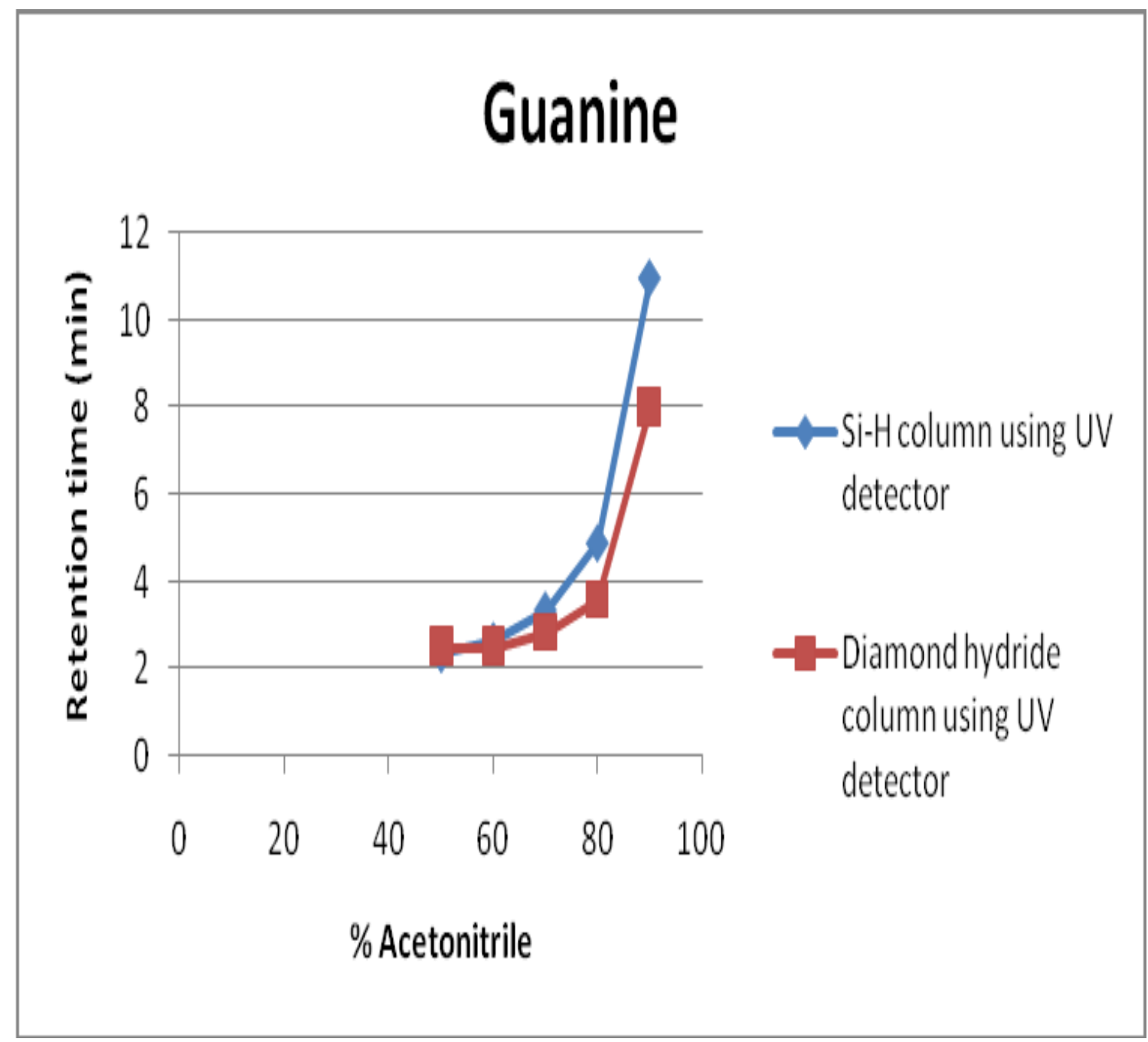

Figure 72. Comparison of retention maps of guanine on the silica hydride and diamond hydride columns using UV detection. 
Figure 73 compares the gradient retention of benzylamine on the silica hydride and diamond hydride columns using Gradient $\mathrm{D}$. As can be seen from the figure, the compound showed very similar retention on both columns. This data indicates a balance between the polar effects of the silica hydride surface and the hydrophobic effects of the diamond hydride are approximately the same resulting in similar retention for benzylamine.
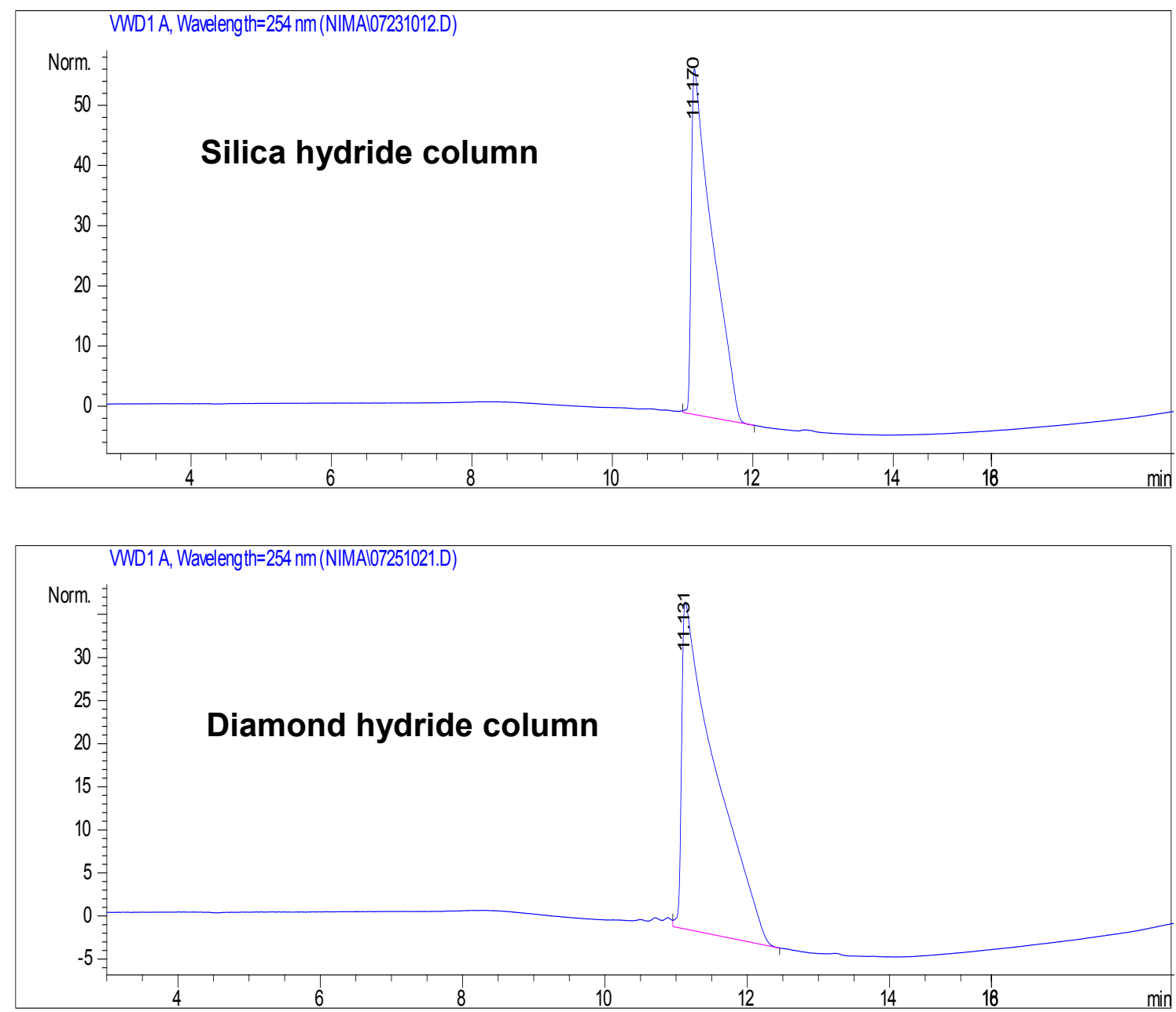

Figure 73. Chromatogram of benzylamine on the silica hydride and diamond hydride columns. Mobile phase Gradient D. 
Figure 74 shows the gradient retention of 4-chloro-1,2-phenylenediamine on the silica hydride and diamond hydride columns. Reasonable peak shape is obtained when using a gradient method (D). It can be seen from the figure that the compound retained slightly more on the silica hydride column compared to the diamond hydride column. Therefore, in the case of this primary amine one can estimate a slightly larger contribution of the silica hydride surface than the carbon on the diamond hydride column.
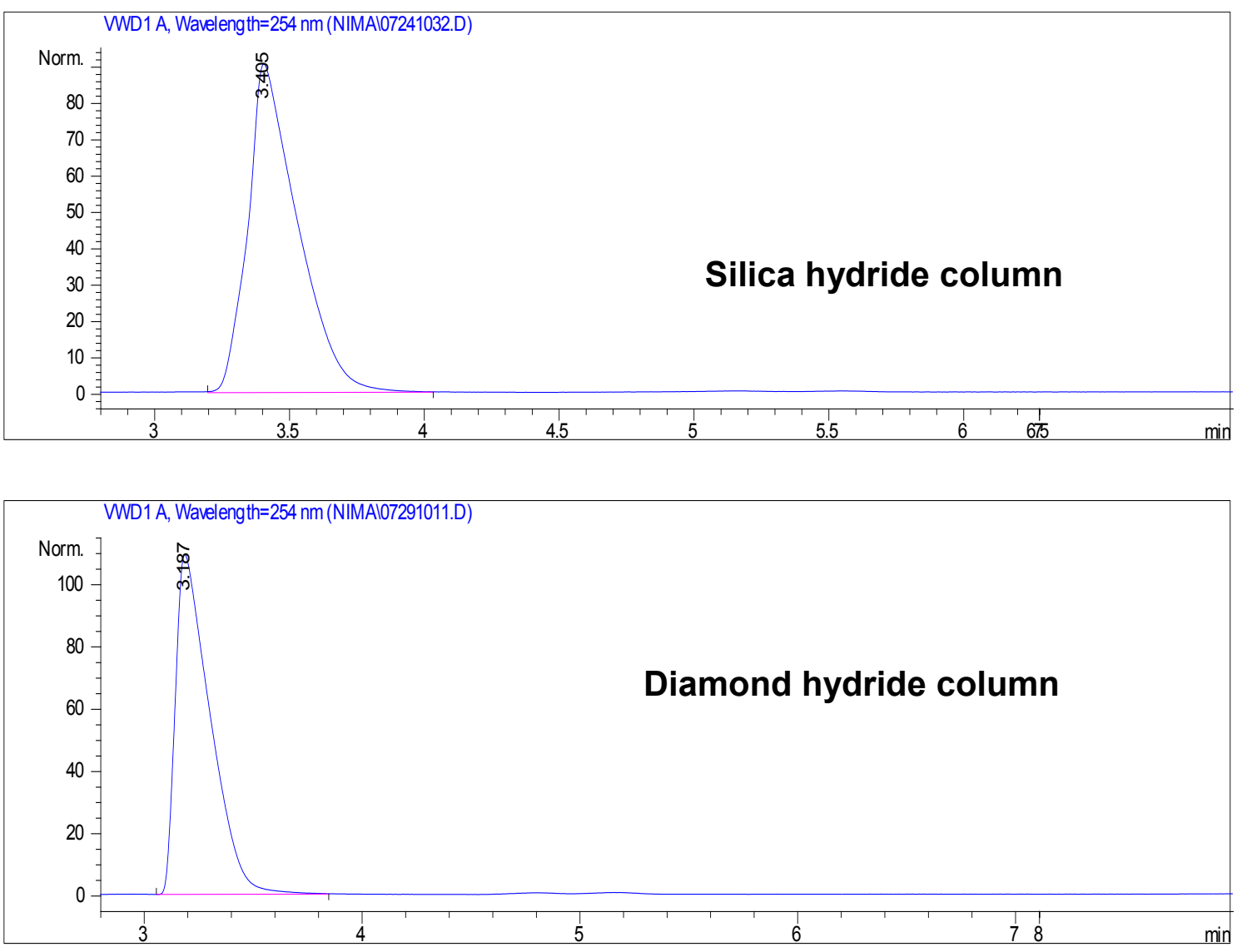

Figure 74. Chromatograms of 4-chloro-1,2-phenylenediamine on the silica hydride and diamond hydride columns. Mobile phase Gradient D. 
Separation of benzylamine and 2,6-diaminopurine on both columns is shown in Figure 75. For both columns the compounds eluted in the same order but with different retention times. The retention time of both primary amines was slightly greater on the diamond hydride column than the silica hydride column. Under these mobile phase conditions the contribution of the small percentage of carbon on the diamond hydride column had a slighly greater effect on retaining these compounds more on this column.
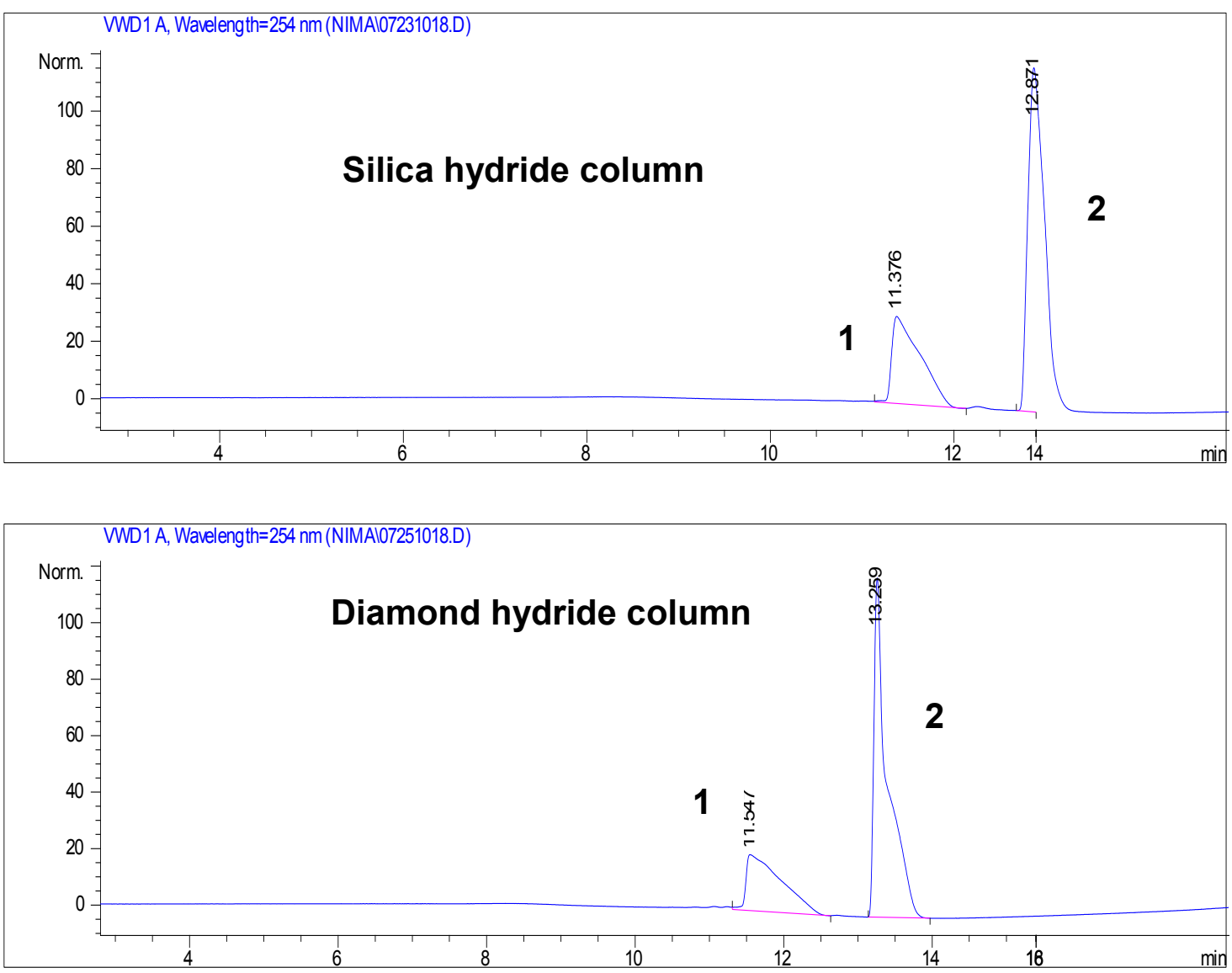

Figure 75. Separation of benzylamine and 2,6-diaminopurine on the silica hydride and diamond hydride columns. Mobile phase Gradient D. 
Figure 76 shows the separation of 1,2,4,5-benzenetetramine tetrahydrochloride, 2-phenethylamine and 2,6-diaminopurine on the silica hydride and diamond hydride columns using Gradient B. For both columns the compounds eluted in the same order but with different retention times. All the compounds showed good retention and were well separated on both columns. It can be observed from the figure that all three compounds retained in the diamond hydride column much longer compared to the silica hydride column, indicating a somewhat greater hydrophobic effect of the carbon on the surface present on the diamond hydride column.

Separation of 1,2,4,5-benzenetetramine tetrahydrochloride and 2phenethylamine on both columns is shown in Figure 77. For both columns, the compounds eluted in the same order but with marginally different retention times. Both compounds showed very similar retention but good separation on both columns. Thus, the contributions of the small percentage of carbon on the diamond hydride surface and the polar silica hydride surface were similar for retaining these compounds.

Figure 78 shows the separation of 1,2,4,5-benzenetetramine tetrahydrochloride and 2,6-diaminopurine on the silica hydride and diamond hydride columns using Gradient A. As can be seen with both columns, the two primary amines eluted in the same order but with different retention times. Being less polar in nature, 1,2,4,5-benzenetetramine tetrahydrochloride eluted first whereas 2,6-diaminopurine being more polar in nature retained longer on the 
column. It can be observed from the figure that 1,2,4,5-benzenetetramine tetrahydrochloride showed very similar retention on both columns whereas 2,6diaminopurine showed longer retention on the diamond hydride column indicating a somewhat greater hydrophobic effect of the carbon on the surface.
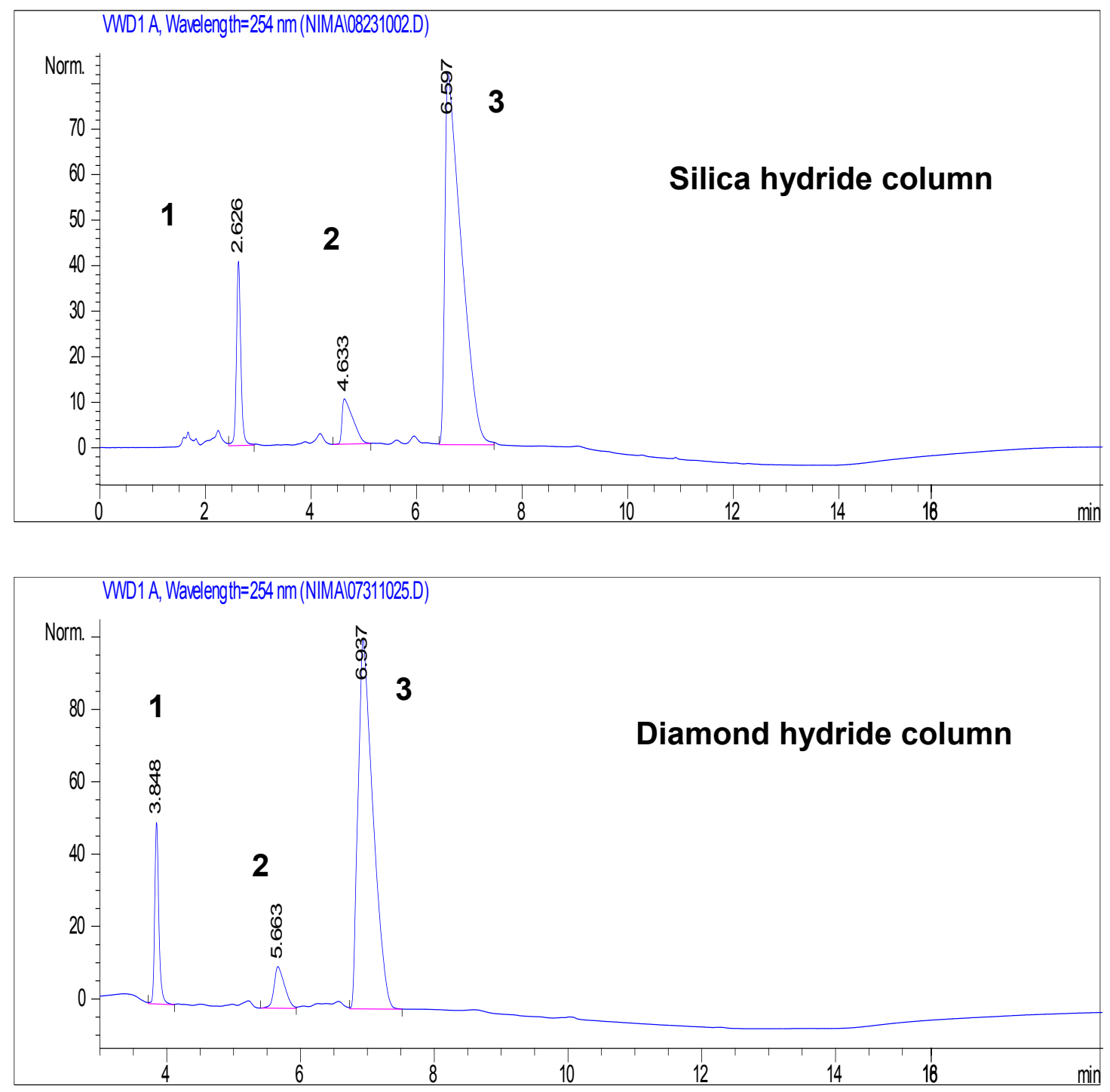

Figure 76. Separation of 1,2,4,5-benzenetetramine tetrahydrochloride, 2phenethylamine and 2,6-diaminopurine on the silica hydride and diamond hydride columns. Mobile phase Gradient B. 

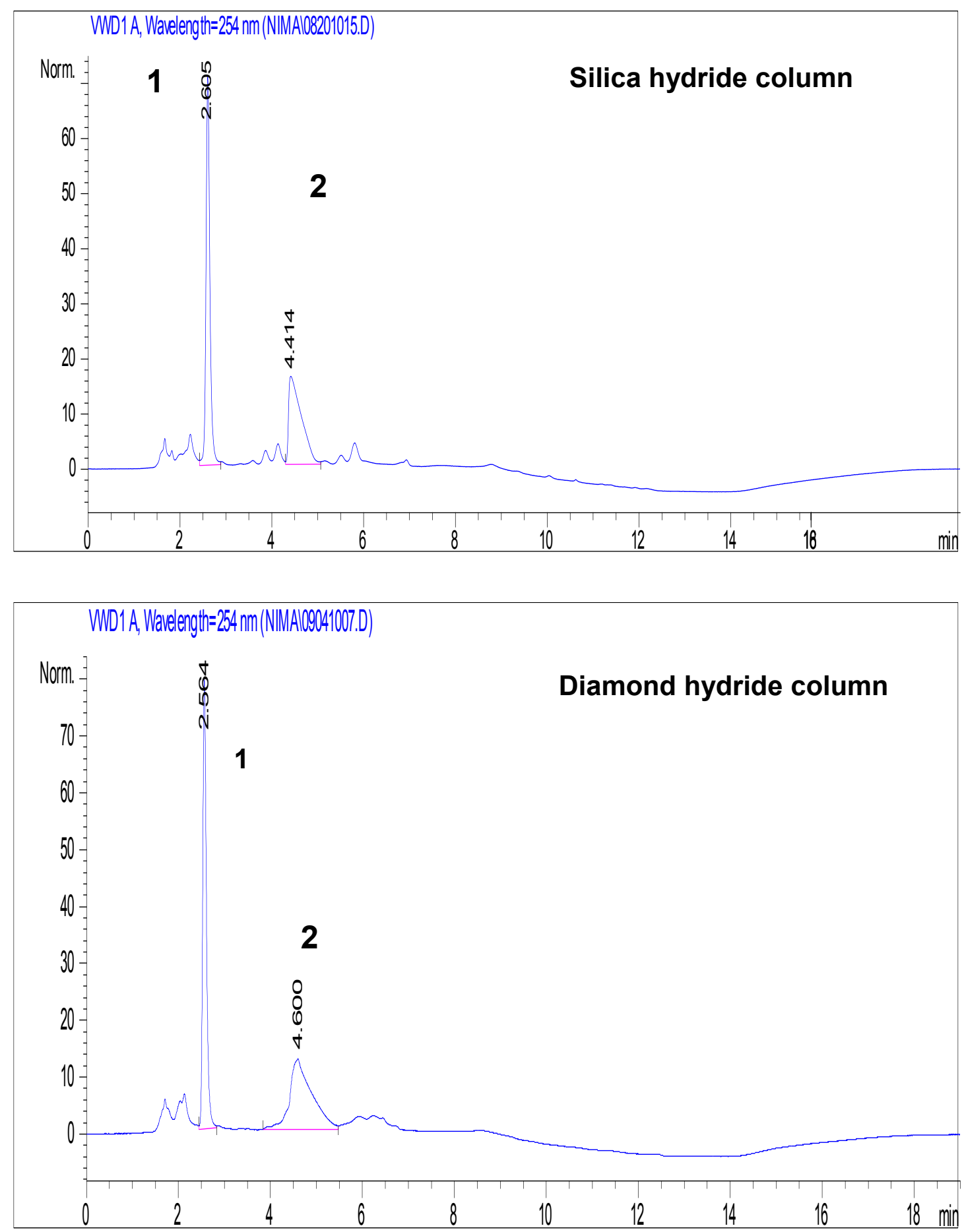

Figure 77. Separation of 1,2,4,5-benzenetetramine tetrahydrochloride and 2phenethylamine on the silica hydride and diamond hydride columns. Mobile phase Gradient B. 

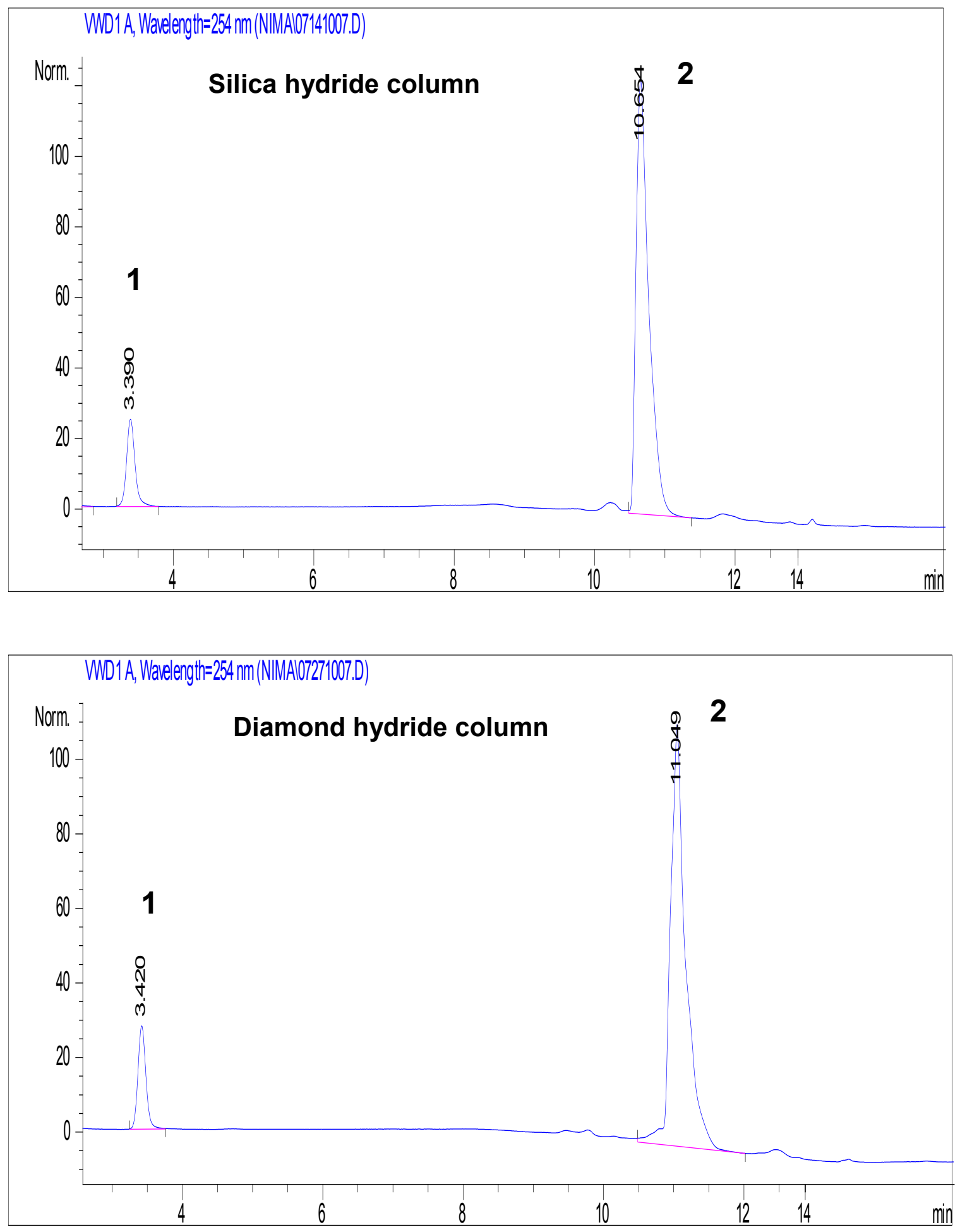

Figure 78. Separation of 1,2,4,5-benzenetetramine tetrahydrochloride and 2,6-diaminopurine on the silica hydride and diamond hydride columns. Mobile phase Gradient A. 


\subsection{Column Efficiency}

The efficiency of a column can be determined by equation 8 . Efficiency is a measure of residence time distribution of a compound passing through the column. Optimum efficiency is calculated for a column which is well packed. A high throughput system running at high velocity is not an ideal environment for calculating efficiency [5]. The research work done here was not under ideal conditions; thus, measured efficiency is not at its best, but an attempt is made to show a comparison between the two columns. Efficiency can be determined by Equation 8.

$$
N=16\left(\frac{t r}{w}\right)^{2}
$$

Where $\mathrm{N}$ is the number of theoretical plates, $\mathrm{t}_{\mathrm{r}}$ is the retention time, and $\mathrm{w}$ is the peak width.

\subsection{Peak Symmetry Factor}

This research of chromatographic characterization of columns also included analysis of the peak symmetry. An ideal peak is symmetric in shape. But unwanted interactions of basic compounds with residual silanols on the stationary phase, non specific adsorption to the stationary phase, contaminated mobile phase or sample can sometimes cause peak tailing effects and 
compounds are retained for a longer period of time in the columns [5]. The peak asymmetry factor can be defined by Equation 9 .

$$
\mathrm{As}=\mathrm{b} / \mathrm{a}
$$

Where As is the asymmetry factor, $a$ and $b$ are determined at $10 \%$ peak height as shown in Figure 79 [5]. A peak is symmetrical in shape if a equals to $b$ and gives asymmetry factor of 1 . The closer the asymmetry factor is to one the better the peak symmetry. A typical acceptable range of asymmetry factor is 0.9 1.2.

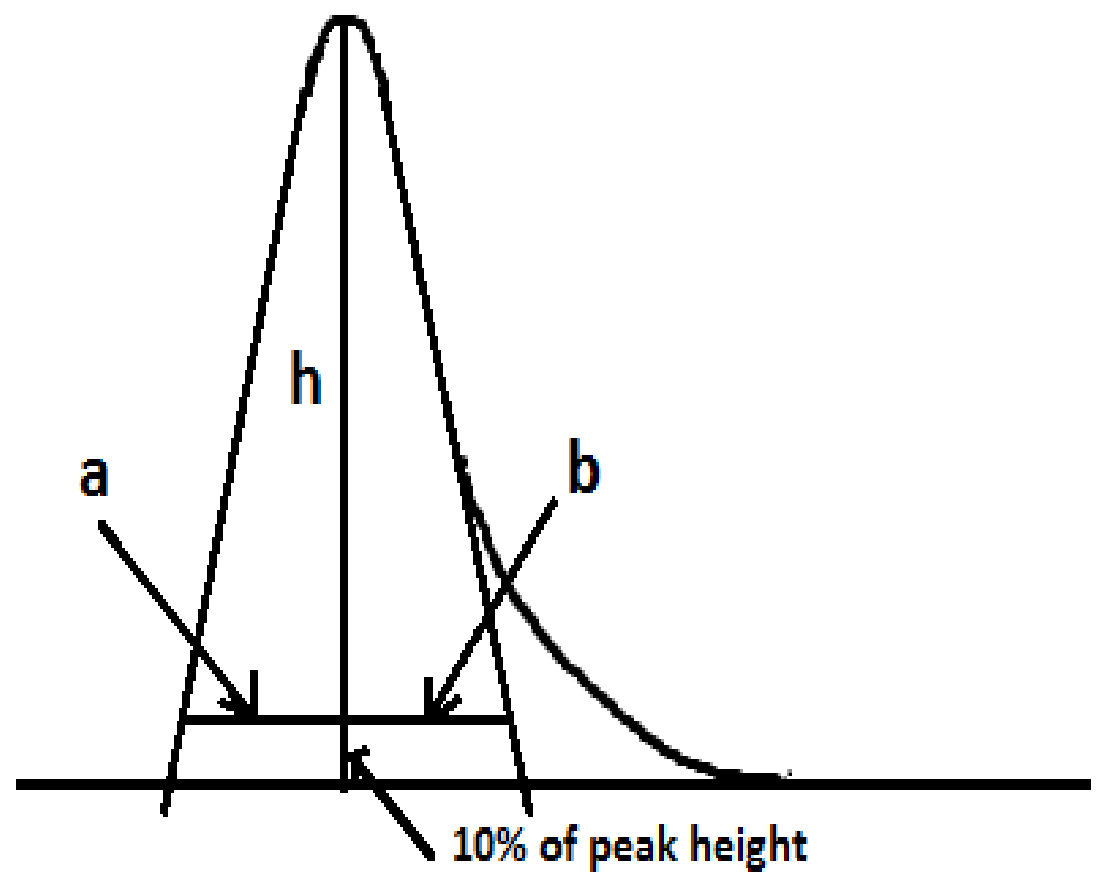

Figure 79. Determination of peak asymmetry factor. 
Figure 80 shows the separation of 2,6-diaminopurine and 2phenethylamine on the silica hydride and diamond hydride columns respectively. Both compounds are well separated on both columns. The symmetry and efficiency were calculated for both compounds and the data are reported in Table 33.

Column efficiency and symmetry factor are relative numbers and depend on the column length, flow rate and particle size. There are also external factors like sample-injection technique, connecting tubing and the detector itself which can affect these two column parameters. The data in Table 33 shows that the silica hydride column had higher efficiency compared to the diamond hydride column. It could be attributed to packing efficiency of the column or the gradient used for 2-phenethylamine and 2,6-diaminopurine could be more suitable for the silica hydride column compared to the diamond hydride column. It can be seen from Table 33 that the symmetry value is higher than 1.8 for 2-phenethylamine and 2,6-diaminopurine on the silica hydride column and 2-phenethylamine on the diamond hydride column. Though these compounds are not symmetrical in the acceptable range, they are well separated. Better symmetry can be achieved by adding modifiers to the mobile phase or by using a different gradient. 

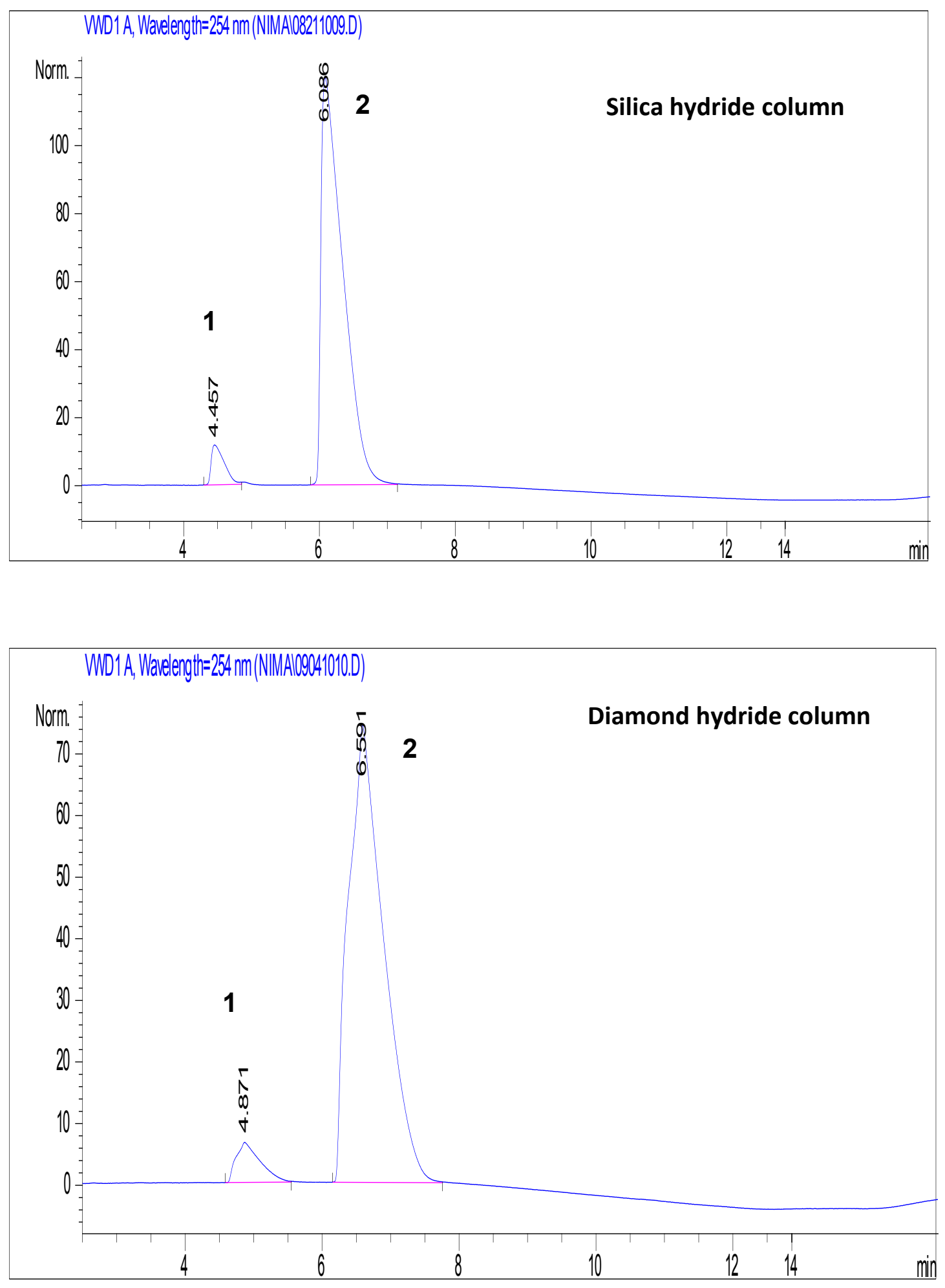

Figure 80. Separation of 2-phenethylamine and 2,6-diaminopurine on the silica hydride and diamond hydride columns. Mobile phase Gradient B. 
Table 33. Comparison of efficiency and asymmetry factor on the silica hydride and diamond hydride columns using UV detection.

\begin{tabular}{|c|c|c|c|c|}
\hline & \multicolumn{2}{|c|}{ Si-H column } & \multicolumn{2}{c|}{ Diamond hydride column } \\
\hline & $\begin{array}{c}\text { 2-Phenethyl- } \\
\text { amine }\end{array}$ & $\begin{array}{c}\text { 2,6- } \\
\text { Diaminopurine }\end{array}$ & $\begin{array}{c}\text { 2-Phenethyl- } \\
\text { amine }\end{array}$ & $\begin{array}{c}\text { 2,6- } \\
\text { Diaminopurine }\end{array}$ \\
\hline $\begin{array}{c}\text { Efficiency(Number of } \\
\text { theoretical plates) }\end{array}$ & 2392.62 & 1776.67 & 547.95 & 636.87 \\
\hline Symmetry & 2.82 & 4.48 & 2.17 & 1.68 \\
\hline
\end{tabular}

Figure 81 shows the separation of 1,2,4,5-benzenetetramine tetrahydrochloride and 2,6-diaminopurine on the silica hydride and diamond hydride columns respectively. Both compounds are well separated on both columns. The symmetry and efficiency were calculated for the separated compounds, and the data are shown in Table 34.

It can be seen from Table 34 that the efficiency and symmetry for silica hydride and diamond hydride column are again moderately good. Both compounds, 1,2,4,5-benzenetetramine tetrahydrochloride and 2,6-diaminopurine are eluting around the same time from both columns but the peak width of each compound is different between the two columns resulting in different efficiency numbers. The peak symmetry numbers are good and acceptable for both columns. Only, 2,6-diaminopurine on the diamond hydride column showed higher symmetry. Again, better symmetry can be achieved by adding some modifiers or by using a different gradient. 

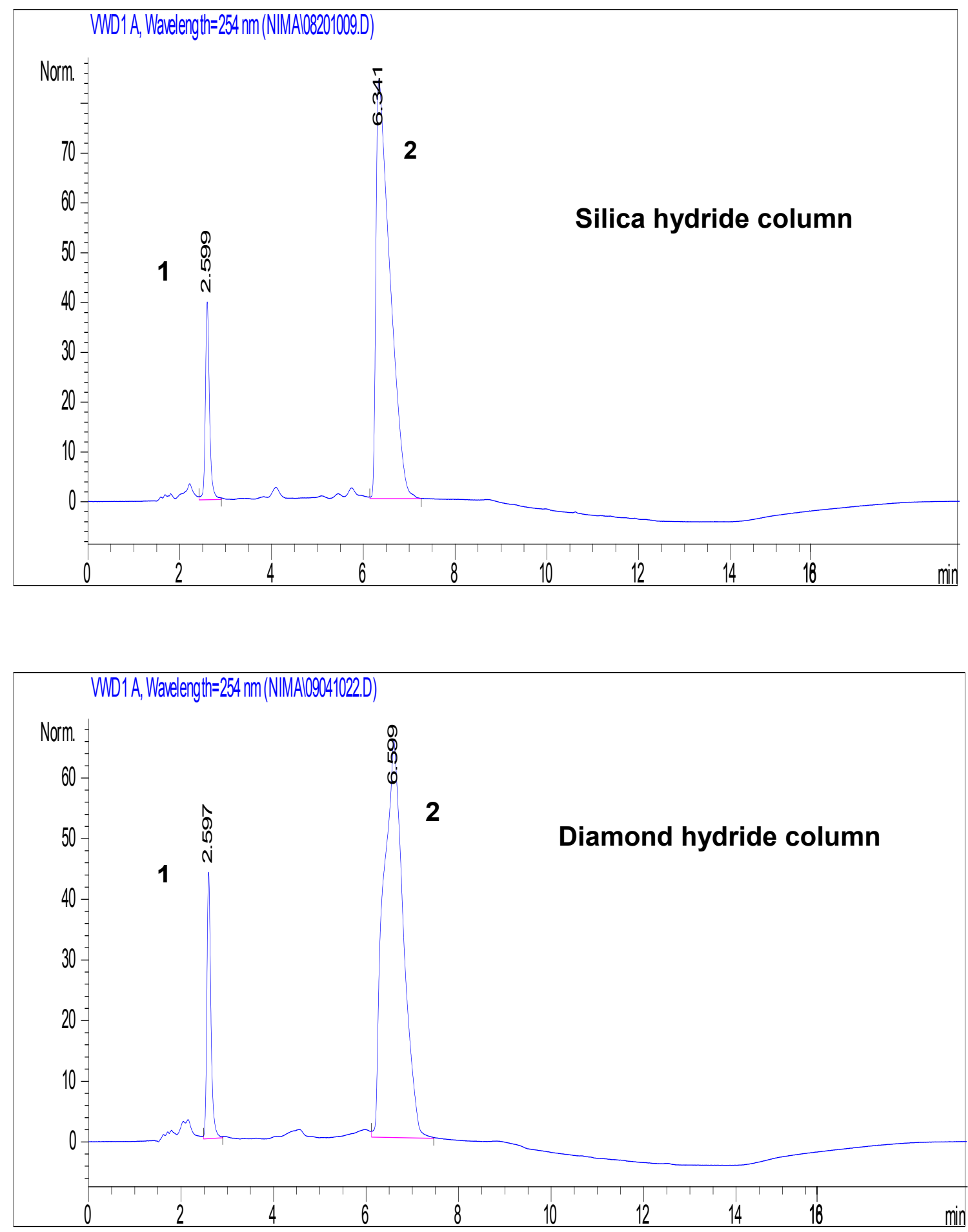

Figure 81. Separation of 1,2,4,5-benzenetetramine tetrahydrochloride and 2,6diaminopurine on the silica hydride and diamond hydride columns. Mobile phase Gradient B. 
Table 34. Comparison of efficiency and asymmetry factor on the silica hydride and diamond hydride columns using UV detection.

\begin{tabular}{|l|c|c|c|c|}
\hline & \multicolumn{2}{|c|}{ Si-H column } & \multicolumn{2}{c|}{ Diamond hydride column } \\
\hline & $\begin{array}{c}\mathbf{1 , 2 , 4 , 5 -} \\
\text { Benzenetetramine- } \\
\text { tetrahydrochloride }\end{array}$ & $\begin{array}{c}\mathbf{2 , 6 -} \\
\text { Diaminopurine }\end{array}$ & $\begin{array}{c}\mathbf{1 , 2 , 4 , 5 -} \\
\text { Benzenetetramine- } \\
\text { tetrahydrochloride }\end{array}$ & $\begin{array}{c}\mathbf{2 , 6 -} \\
\text { Diaminopurine }\end{array}$ \\
\hline $\begin{array}{c}\text { Efficiency } \\
\text { (Number of } \\
\text { theoretical } \\
\text { plates) }\end{array}$ & 4634.77 & 1827.48 & 5140.33 & 976.73 \\
\hline Symmetry & 1.09 & 4.76 & 1.12 & 1.33 \\
\hline
\end{tabular}




\section{Conclusions}

Silica hydride and diamond hydride columns have shown significant retention of amino acids, nucleosides, nucleobases, ergogenic acids, and primary amines. These compounds were analyzed using HPLC with UV and ELSD detectors. To understand the retention mechanism exhibited by the silica hydride and diamond hydride columns for different polarity compounds, ANP retention maps of some of the compounds were compared. Guanidine, creatinine, and guanine were retained longer on the silica hydride column indicating the ANP retention was due to the slightly more polar surface than that of the diamond hydride column. L-Tryptophan and adenosine retained much longer on the diamond hydride column which had small percentage of carbon on the surface, responsible for the hydrophobic retention.

Both columns showed very good separation of primary amines compounds. The results obtained on the silica hydride column for the separation of all polar compounds used in this research were comparable with those obtained from the diamond hydride column. For the separation of primary amines, different gradient elution modes were used with a UV detector. The primary amines, benzylamine, 2-phenethylamine, 1,2,4,5-benzenetetramine tetrahydrochloride, and 4-chloro 1,2-phenylenediamine showed very similar retention on both columns, indicating the balance between the polar effects of the silica hydride surface and hydrophobic effect of the diamond hydride column. The compound 2,6-diaminopurine showed longer retention on the diamond hydride 
column indicating the contribution of the small percentage of carbon on the diamond hydride column. The peak symmetry factors and column efficiency parameters were calculated for selected compounds. The primary amine, 2,6diaminopurine showed very good symmetry on the diamond hydride column of around 1.6 or better but poor efficiency compared to the silica hydride column. 2Phenethylamine showed better efficiency on silica hydride compared to diamond hydride but comparable symmetry between the two. Whereas $1,2,4,5-$ benzenetetramine tetrahydrochloride showed much better efficiency as well as symmetry on the diamond hydride column compared to the silica hydride column. These results suggest that both columns showed different peak symmetry and column efficiency for each compound due to their different selectivity or affinity towards each compound. A minor addition of the $2 \%$ carbon on the diamond hydride stationary phase is most likely responsible for the different selectivity and retention of the two stationary phases.

The study suggests that primary amines can be well separated on silica hydride and diamond hydride column using a UV detector. Comparison of efficiency and peak symmetry results suggest that future work can focus on improving efficiency and peak symmetry by using different gradient methods, different mobile phases and additives. 


\section{References}

1. Skoog, A. D.; Hooler, F. J.; Nieman, T. A. Principles of Instrumental Analysis, 5th ed.; Saunders College Publishing: Florida, 1998; Chapters 26-28.

2. Snyder, L. R.; Kirkland, J. J.; Glajch, J. L. Practical HPLC Method Development. 2nd ed.; John Wiley and Sons, New York, 1997, Chapters 3, 5, 8, 11.

3. Pesek, J. J.; Matyska, M. T. LCGC NORTH AMERICA. 2006, 24, 296-303.

4. Pesek, J. J.; Matyska, M. T. LCGC NORTH AMERICA. 2007, 30, 637-647.

5. Cunico, R. L.; Gooding, M. M.; Wehr, T. Basic HPLC and CE of Biomolecules, Bay Bioanalytical Laboratory, CA, 1998.

6. The Chromatography introductory theory homepage. http://teaching.shu.ac.uk/hwb/chemistry/tutorials/chrom/chrom1.htm (Accessed October 27, 2012).

7. Karen, M. G.; Fred E.R. HPLC of Biological Macromolecules. Eds.; Marcel Dekker, Inc.: New York, 1990; Chapters 6, 9-11.

8. Pesek, J. J.; Matyska, M. T. J. Chromatogra. A. Sep. Sci. 2005, 28, 1845-1854.

9. Nawrocki, J. J. Chromatogra. A. 1997, 779, 29-71.

10. Pesek, J. J.; Tang, V. H. Chromatographia, 1994, 39, 649-654.

11. Suryadevara, R. Synthesis and characterization of amino based columns for HPLC. M.S. Thesis, San Jose State University, CA, 2004.

12. Dorsey, J. G.; Cooper, W. T. Anal. Chem. 1994, 66, 857A-867A.

13.Pesek, J. J.; Matyska, M. T.; Sandoval, J. E.; Williamsen, E. J. J. Liq. Chromatogr. \& Rel. Technology. 1996, 19, 2843-2865. 
14. Chu, C. H.; Johnson, E.; Auvinen, M.; Pesek, J. J.; Sandoval, E. J. Anal. Chem. 1993, 65, 808-816.

15.Pesek, J. J.; Matyska, M. T. J. Liq. Chromatogra. \& Rel. Technology. 2006, 29, 1105-1124.

16. Sandoval, J. E.; Pesek, J. J. Anal. Chem. 1991, 63, 2634-2641.

17.Pesek, J. J.; Matyska, M. T. J. Chromatogra. A. 2009, 32, 3999-4011.

18. Venkat, J.P. Synthesis and evaluation of a short chain alkyl stationary phase using ELSD. M.S. Thesis, San Jose state University, CA, 2007.

19. Yan, D.; Li, G.; Xiao, X. H.; Dong, X. P.; Li, Z. L. J. Chromatogra. A. 2007, 1138, 310-304.

20. SOFTA CORPORATION, Model 400 ELSD Manual, version 1.2, August 2006. http://www.softacorporation.com (Accessed October 10, 2012).

21.Pesek, J.J.; Matyska, M. T.; Hearn, M. T. W.; Boysen, R. I. J.Chromatogra. A. 2009, 1216, 1140-1146.

22. Kay Ernest, R. M.; Biochemistry- An Introduction to Dynamic Biology. Macmillan Company: NY, 1996; Chapter 26, 342.

23. Wolinsky. I.; Driskell. J. Nutritional Ergogenic Acids, CRC Press: NY, 2004; Chapter 6, 81-104.

24. McMurry, J. E. Organic Chemistry, 6th ed.; Brooks Cole Publishing Company: CA, 2004, Chapter 24, 892.

25. Young J. E. Pesek, J. J.; Matyska, M. T. J. Chromatogra. A. 2010, 1218, 2121-6.

26. Pesek, J. J.; Matyska, M. T.; Fischer, S. M.; Sana, T.R. J. Chromatogra. A. 2008, $1205,48-55$. 